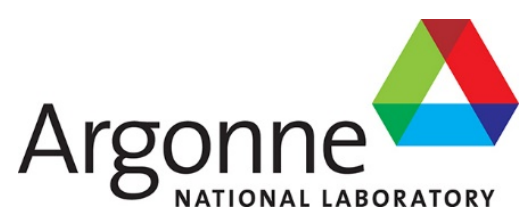

\title{
Multiphysics Coupling of PROTEUS-NODAL and SAM for Molten Salt Reactor Simulation
}

Nuclear Science and Engineering Division 


\begin{abstract}
About Argonne National Laboratory
Argonne is a U.S. Department of Energy laboratory managed by UChicago Argonne, LLC under contract DE-AC02-06CH11357. The Laboratory's main facility is outside Chicago, at 9700 South Cass Avenue, Argonne, Illinois 60439. For information about Argonne and its pioneering science and technology programs, see www.anl.gov.
\end{abstract}

\title{
DOCUMENT AVAILABILITY
}

Online Access: U.S. Department of Energy (DOE) reports produced after 1991 and a growing number of pre-1991 documents are available free via DOE's SciTech Connect (http://www.osti.gov/scitech/)

\author{
National Technical Information Service (NTIS): \\ U.S. Department of Commerce \\ National Technical Information Service \\ 5301 Shawnee Rd \\ Alexandra, VA 22312 \\ www.ntis.gov \\ Phone: (800) 553-NTIS (6847) or (703) 605-6000 \\ Fax: (703) 605-6900 \\ Email: orders@ntis.gov
}

Reports not in digital format may be purchased by the public from the

Reports not in digital format are available to DOE and DOE contractors from the

Office of Scientific and Technical Information (OSTI):

U.S. Department of Energy

Office of Scientific and Technical Information

P.O. Box 62

Oak Ridge, TN 37831-0062

www.osti.gov

Phone: (865) 576-8401

Fax: (865) 576-5728

\begin{abstract}
Disclaimer
This report was prepared as an account of work sponsored by an agency of the United States Government. Neither the United States Government nor any agency thereof, nor UChicago Argonne, LLC, nor any of their employees or officers, makes any warranty, express or implied, or assumes any legal liability or responsibility for the accuracy, completeness, or usefulness of any information, apparatus, product, or process disclosed, or represents that its use would not infringe privately owned rights. Reference herein to any specific commercial product, process, or service by trade name, trademark, manufacturer, or otherwise, does not necessarily constitute or imply its endorsement, recommendation, or favoring by the United States Government or any agency thereof. The views and opinions of document authors expressed herein do not necessarily state or reflect those of the United States Government or any agency thereof, Argonne National Laboratory, or UChicago Argonne, LLC.
\end{abstract}


ANL/NSE-20/7

\section{Multiphysics Coupling of PROTEUS-NODAL and SAM for Molten Salt Reactor Simulation}

prepared by

M. K. Jaradat, G. Yang, H. Park, and W. S. Yang

Department of Nuclear Engineering and Radiological Sciences

University of Michigan

C. H. Lee and Y. S. Jung

Nuclear Science and Engineering Division

Argonne National Laboratory

February 28, 2020 



\section{EXECUTIVE SUMMARY}

In order to enhance the accuracy and efficiency of the PROTEUS-NODAL code for MSR simulation, several new capabilities have been implemented in the variational nodal $\mathrm{P}_{1}$ solver and the PROTEUS-NODAL code has been coupled with the system analysis module SAM. First of all, to eliminate the error caused by approximating a cylindrical MSR core by a hexagonal core, a variational nodal $\mathrm{P}_{1}$ method was developed for cylindrical geometries. The developed capability was verified against the FDM solver of the DIF3D code using 2D and 3D steady state problems derived from the MSFR benchmark. Relative to the fine hexagonal geometry model that yields a comparable accuracy, the computational time was reduced about 100 times by an R-Z model and about 7 to 8 times by an R- $\theta-\mathrm{Z}$ model. Although the computational gain depends on the specific problem, it is obvious that a cylindrical geometry model is more efficient and accurate than a hexagonal geometry model for cylindrical geometry problems.

The transient analysis capability that had been limited to the $\mathrm{SP}_{3}$ solver in hexagonal geometry was extended to the $\mathrm{P}_{1}$ solvers for Cartesian, triangular, hexagonal, and cylindrical geometries. The TFSP solver was extended to the $\mathrm{P}_{1}$ solvers, and a FDM solver for the delayed neutron precursor equation was developed. To improve the computational efficiency for transient analyses, a CMFD acceleration scheme was also implemented. A capability to calculate the kinetics parameters in flowing fuel reactors was also added by implementing a steady state adjoint equation solver. The developed $\mathrm{P}_{1}$ transient solver was verified against the $\mathrm{SP}_{3}$ transient solver that was verified last year. The test results showed that the $\mathrm{P}_{1}$ solutions agree very well with the $\mathrm{SP}_{3}$ solutions. These tests also showed that the CMFD acceleration scheme reduces the computational time for TFSP a few tens of times. The $\mathrm{P}_{1}$ transient solver in $\mathrm{R}-\mathrm{Z}$ geometry was also tested by comparing the results with those of the hexagonal geometry option of the $\mathrm{P}_{1}$ solver and those of the $\mathrm{SP}_{3}$ solver. Various unprotected transient scenarios of the MSFR benchmark problem were solved with thermal feedback, including UTOP, UPOS, ULOF, ULOHS, and UFSOC accidents. Despite the differences in geometrical models and transport approximations (diffusion vs. $\mathrm{SP}_{3}$ ), the three solutions showed very good agreement in the power and core-averaged fuel salt temperature. With comparable accuracies, the $\mathrm{P}_{1}$ solver in $\mathrm{R}-\mathrm{Z}$ geometry reduces the computational time about 10 to 60 times relative to the $\mathrm{SP}_{3}$ solver and about 5 to 10 times relative to the $\mathrm{P}_{1}$ solver in hexagonal geometry.

In order to enhance the thermal-hydraulics modeling capabilities by overcoming the limitation of the standalone thermal-hydraulics solver, PROTEUS-NODAL has been coupled with SAM under the MOOSE framework. A MOOSE sub-application named TreeFrog was developed as the wrapper for PROTEUS-NODAL to communicate with other MOOSE applications. A MOOSE master application named TreeKangaroo was also developed to control the coupling calculation of TreeFrog and SAM. Different time step sizes between PROTEUS-NODAL and SAM are allowed in the coupled transient calculations by using the "subcycling" option of the MOOSE transient executioner. The Picard iteration was used in the coupled steady state calculation, and the operator-splitting method was used in the coupled transient calculations. 
Verification tests of the coupled system of PROTEUS-NODAL and SAM were performed using the steady state and transient problems derived from the MSFR benchmark problem. Since the radial crossflow is neglected in the current SAM model, the effect of this simplification was first examined by comparing the steady state results with those obtained by a manually coupled calculation of PROTEUS-NODAL and ANSYS CFX. The SAM calculation used four parallel axial channels and CFX performed the full 3D CFD calculation in the cylindrical geometry of the MSFR core. Due to the neglect of the radial velocity field in the SAM calculation, SAM underestimated the axial velocity at the core center slightly, and this resulted in a slightly top-skewed power distribution: $0.1 \%$ overestimation in the upper part and $0.2 \%$ underestimation in the lower part of the core. The UTOP, ULOF, and ULOHS accidents of the MSFR transient benchmark were analyzed by including the outer loop in the SAM model. The results were compared with the PSI solutions from a coupled PARCS and TRACE calculation and the TUDelft solutions obtained from a coupled neutron diffusion and CFD calculation. In general, the power and core-averaged fuel temperature solutions of the coupled PROTEUS-NODAL and SAM calculations agreed well with the other solutions. 


\section{REVISION HISOTRY}

\begin{tabular}{|c|l|}
\hline Revision & \multicolumn{1}{c|}{ Description } \\
\hline 0 & $\begin{array}{l}\text { Release date: February 28, 2020 } \\
\text { Initial release }\end{array}$ \\
\hline 1 & $\begin{array}{l}\text { Release date: April 10, 2020 } \\
\text { Updates } \\
-\quad \text { TreeFlog/TreeKangaroo coupling with SAM 3D }\end{array}$ \\
\hline
\end{tabular}




\section{TABLE OF CONTENTS}

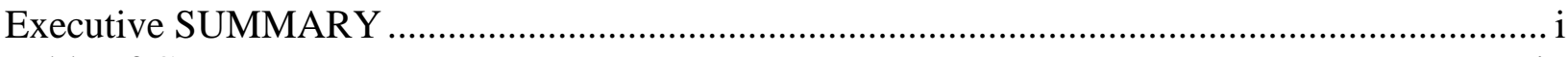

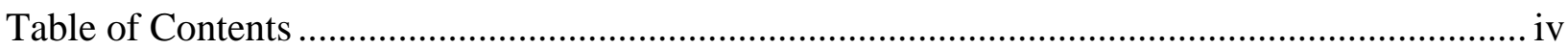

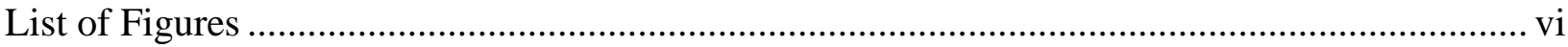

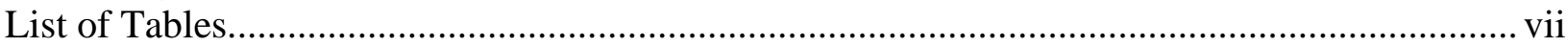

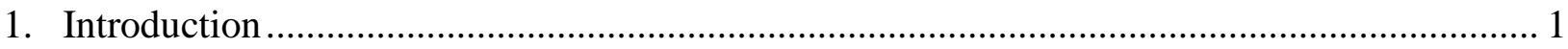

2. Development of Variational Nodal $\mathrm{P}_{1}$ Solver in R- $\theta-\mathrm{Z}$ Geometry …....................................... 4

2.1. Variational Nodal Method for Diffusion Theory ………………….................................. 4

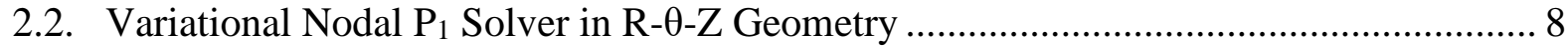

2.2.1. Generation of Basis Functions......................................................................... 8

2.2.2. Generation of Response Matrices........................................................................ 10

2.2.3. Boundary Conditions in $\theta$ Direction........................................................................ 11

2.3. Verification Tests of Variational Nodal $P_{1}$ Solver for R- $\theta-Z$ Geometry........................ 11

2.3.1. R-Z Geometry Problem ........................................................................................ 11

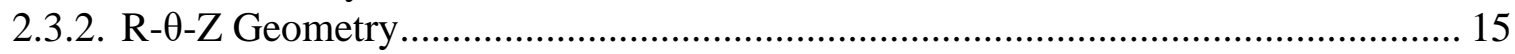

3. Development of Transient Analysis Capabilities of $\mathrm{P}_{1}$ Solver ............................................... 18

3.1. Transient Fixed Source Problem ................................................................................ 18

3.2. Solution of Delayed Neutron Precursor Equation for Flowing Fuel ............................. 20

3.3. Implementation of CMFD Acceleration in Variational Nodal $P_{1}$ Solver ....................... 22

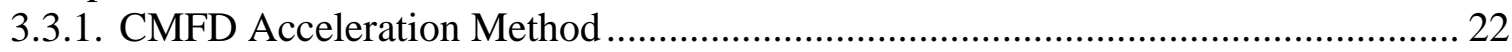

3.3.2. Performance of CMFD Acceleration................................................................. 23

3.4. Evaluation of Kinetics Parameters .............................................................................. 25

3.4.1. Adjoint Flux and Kinetics Parameters................................................................. 25

3.4.2. Verification Tests of Kinetics Parameters ........................................................... 27

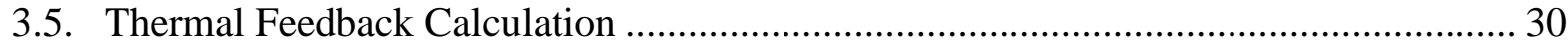

3.5.1. Cross Section Functionalization ........................................................................... 30

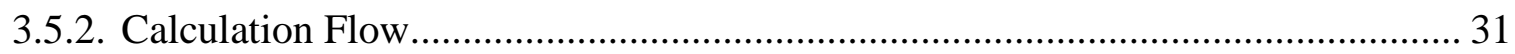

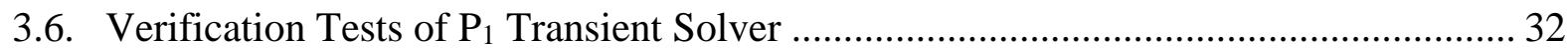

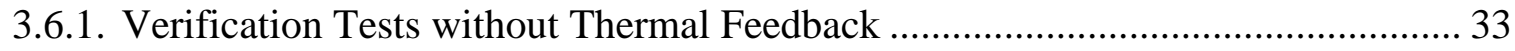

3.6.2. Verification Tests with Thermal Feedback ……………………………………..... 36

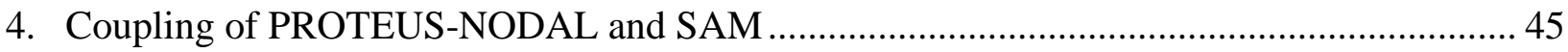

4.1. Coupled Calculation Scheme ……………………...................................................... 45

4.1.1. PROTEUS-NODAL Wrapper TreeFrog ................................................................. 46

4.1.2. Overall Computational Procedure of TreeKangaroo................................................ 48

4.2. Verification Test Results............................................................................................... 52

4.2.1. Comparison of Steady State Results of SAM with ANSYS CFX.......................... 52

4.2.2. Transient Tests of Coupled System of PROTEUS-NODAL and SAM .................. 56

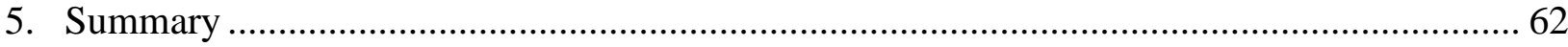

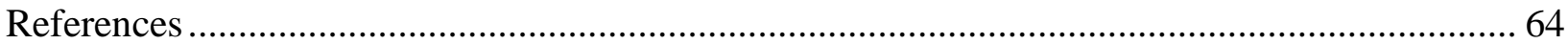

Appendix A. Description of MSFR Benchmark Problem ................................. 67

Appendix B. Comparison of Three Solution Methods for Delayed Neutron Precursor Equation of Flowing Fuel ................................................... 70

Appendix C. Standard MSR Thermal-Hydraulics Solver of PROTEUS ...................... 78

Appendix D. PROTEUS-NODAL Input Data for MSR Analysis ............................. 102 
Multiphysics Coupling of PROTEUS-NODAL and SAM for Molten Salt Reactor Simulation

February 28, 2020

Appendix E. Input Data for TreeFrog and TreeKangaroo Applications ....................... 109 


\section{LIST OF FIGURES}

Fig. 2.1. Simplified R-Z geometry of MSFR.............................................................. 12

Fig. 2.2. MSFR core models in original cylindrical geometry (left) and in approximate hexagonal geometry of 8 rings of hexagons of $16.5 \mathrm{~cm}$ pitch (right)........................ 13

Fig. 2.3. Approximated hexagonal geometry core models of MSFR with 12 rings of hexagons of $11.3 \mathrm{~cm}$ (left) and 15 rings of hexagons of $9.0 \mathrm{~cm}$ pitch (right) ........................... 13

Fig. 2.4. Cross sectional view at an azimuthal angle where control rod is inserted in the simplified MSFR. 16

Fig. 2.5. Modified core models with control rods in $\mathrm{R}-\theta-\mathrm{Z}$ geometry with $\Delta \theta=13.3^{\circ}$ for the control rod regions and $17.8^{\circ}$ for other regions (left) and in hexagonal geometry with 15 rings of hexagons of $9.0 \mathrm{~cm}$ pitch (right).......................................................... 16

Fig. 3.1. Comparison of error reduction in fission source with and without CMFD for eigenvalue problems of (a) small and (b) large MSFR cores

Fig. 3.2. Comparison of (a) the computational time and (b) the number of outer iterations at each time step with and without CMFD for the modified TWIGL benchmark problem.

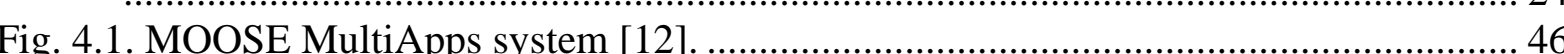

Fig. 4.2. Axial nodes of PROTEUS-NODAL and axial meshes of TreeFrog. ...................... 48

Fig. 4.3. Data flow and coupling scheme between PROTEUS-NODAL and SAM under the

MOOSE framework.

Fig. 4.4. Coupling scheme with Picard iteration for steady state calculations......................... 50

Fig. 4.5. Coupling scheme with operator splitting for transient calculations. ....................... 51

Fig. 4.6. Axial (left) and radial (right) velocity fields of the fuel salt obtained from CFX. The

cylinder axis is in the middle of both figures.

Fig. 4.7. Comparison of axial velocity profiles of SAM and CFX..................................... 54

Fig. 4.8. (a) Power distribution (W/cc) of coupled PROTEUS-NODAL and SAM calculation and (b) \% difference from that of coupled PROTEUS-NODAL and CFX calculation.

Fig. 4.9. Comparison of axial temperature profiles of SAM and CFX................................. 55

Fig. 4.10. SAM model of the MSFR benchmark problem.................................................. 56

Fig. 4.11. Power evolution during the UTOP transient. ................................................... 57

Fig. 4.12. Average core temperature increase during UTOP transient. ................................ 58

Fig. 4.13. Power evolution during ULOF transient. ............................................................... 59

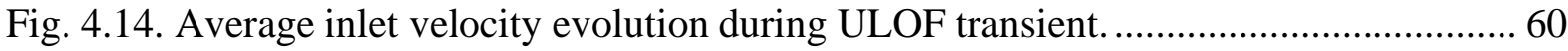

Fig. 4.15. Power evolution during ULOHS transient. ..................................................... 60

Fig. A.1. Schematic representation of the molten salt fast reactor. ................................... 67

Fig. B.1. Illustration of characteristic lines for precursor concentration calculation.............. 71

Fig. B.2. Fuel salt path at each time step from a starting point to a node outlet.................... 73

Fig. B.3. Power evolution in null transient without thermal feedback. ............................... 76

Fig. B.4. Power evolution in pump start-up transient without thermal feedback. .................. 76

Fig. B.5. Power evolution in pump coast-down transient without thermal feedback.............. 77

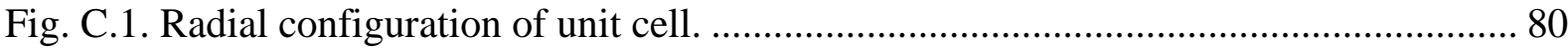

Fig. C.2. Main and momentum control volumes and state variables................................... 83

Fig. C.3. Mesh structure for heat conduction calculations............................................... 84

Fig. C.4. Schematic diagram of the heat removal from the primary system of MSFR........... 98 
Fig. D.1. Example of a driver input file........................................................................... 106

Fig. D.2. Example of a material assignment file................................................................ 107

Fig. D.3. Example of an assignment file for standalone thermal-hydraulics calculation. ..... 108

Fig. E.1. Sample input for TreeKangaroo. ....................................................................... 123

Fig. E.2. Sample input for TreeFrog. ............................................................................ 124

\section{LIST OF TABLES}

Table 2.1. Surface Numbering Scheme in the R- $\theta-Z$ Geometry ................................................. 9

Table 2.2. Geometry Dependent Matrix Scaling Factors............................................................... 11

Table 2.3. Eigenvalue and Power Comparison Results of Unrodded Case .............................. 14

Table 2.4. Eigenvalue and Power Comparison Results of Rodded Case................................... 17

Table 3.1. Performance of CMFD for Eigenvalue Problems..................................................... 23

Table 3.2. Calculated Safety Parameters for MSFR Benchmark Problem ............................... 28

Table 3.3. Eigenvalues of Steady State MSFR Benchmark Problem ....................................... 37

Table 3.4. Performance Comparison of Three Solvers for MSFR Transient Problems ........... 44

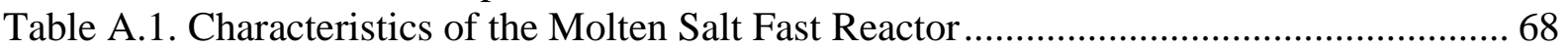

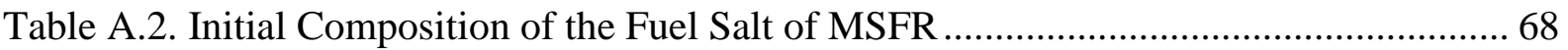

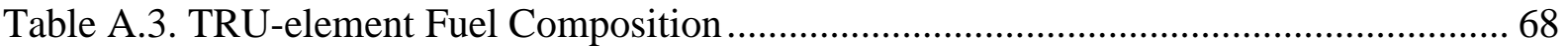

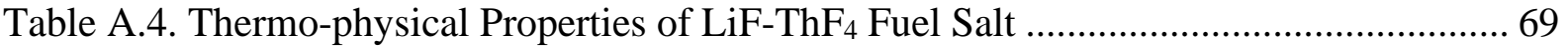

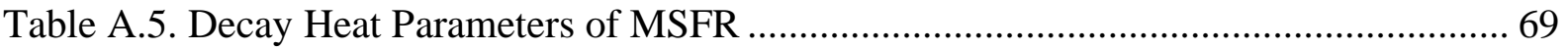

Table D.1. Input Cards in Driver Input File........................................................................... 102

Table D.2. Keywords Added to Geometry Input for R- $\theta-Z$ Geometry .................................... 103

Table D.3. Input Cards in Material Assignment File ............................................................. 103

Table D.4. Input Cards in Standalone Thermal-Hydraulics Input File ................................... 104

Table E.1. Input Description of Mesh Block ………….......................................................... 109

Table E.2. Input Description of AuxVariables Block ……………….................................... 110

Table E.3. Input Description of Function Block ..................................................................... 110

Table E.4. Input Description for Problem Block in TreeKangaroo ......................................... 111

Table E.5. Input Description for Problem Block in TreeFrog ............................................... 112

Table E.6. Input Description for Steady Executioner Block..................................................... 113

Table E.7. Input Description for SteadyWithPicardCheck Executioner Block ....................... 113

Table E.8. Input Description for Transient Executioner Block ............................................. 113

Table E.9. Input Description for MultiApps Block .............................................................. 114

Table E.10. Input Description for Transfers Block.............................................................. 115

Table E.11. Input Description for Outputs Block with Short-cut Syntax .............................. 117

Table E.12. Input Description for Outputs Block with Sub-block Syntax ............................ 117 



\section{Introduction}

The molten salt reactor (MSR) with the fuel dissolved into the liquid salt has been selected as one of the six Generation-IV reactors due to its excellent characteristics in the aspects of sustainability, economy, passive safety, and resource utilization [1-3]. In a liquid fuel MSR, the fuel salt is circulated throughout the primary system and thus some of the delayed neutron precursors generated in the core decay in positions of low importance or even out of the core. The MSR can be configured in both thermal and fast spectrum reactors, depending on the use of a moderator. Thermal spectrum MSRs such as the Molten Salt Reactor Experiment (MSRE) and the Molten Salt Breeder Reactor (MSBR) design use the graphite moderator and the fuel flows through the channels in the graphite moderator while fast spectrum MSRs such as the Molten Salt Fast Reactor (MSFR) design have no moderator.

Recently both thermal and fast spectrum MSRs are gaining increasing interest from the industry. This increases the need for advanced design analysis capabilities for MSR applications. In order to meet this need for MSR modeling and simulation capabilities, it was decided to extend the capabilities of the PROTEUS-NODAL code [4,5] being developed at Argonne National Laboratory for the steady state and transient analyses of MSRs. PROTEUS-NODAL is a three-dimensional (3D) neutron transport code based on a homogeneous assembly model for various nuclear reactor applications. The code has two solution options: the $\mathrm{P}_{1}$ solver based on the DIF3D-VARIANT methodology [6] and the simplified $\mathrm{P}_{3}\left(\mathrm{SP}_{3}\right)$ solver based on the triangle-based polynomial expansion nodal (TPEN) method for the radial direction and the one-dimensional nodal expansion method (NEM) for the axial direction [7]. The $\mathrm{P}_{1}$ solver is applicable to Cartesian, triangular, and hexagonal geometry problems, while the $\mathrm{SP}_{3}$ solver is applicable to hexagonal geometry problems only.

Last year, both the $\mathrm{P}_{1}$ and $\mathrm{SP}_{3}$ solvers were extended to model MSRs by taking into account the precursor drift in a flowing fuel [8-10]. However, the transient analysis capability was limited to the $\mathrm{SP}_{3}$ solver for hexagonal geometry problems. As a result, cylindrical cores of molten salt fast reactors had to be approximated by hexagonal geometry models. In addition, the standalone thermal-hydraulics solver implemented in the PROTEUS-NODAL code was based on a parallel one-dimensional (1D) multi-channel model for the core and simple lumped parameter models for the components outside of the core [10]. In order to improve the analysis accuracy by eliminating the unnecessary modeling approximations, it was decided to extend the $\mathrm{P}_{1}$ solver to cylindrixal geometry problems and to couple the PROTEUS-NODAL code with the System Analysis Module (SAM) [11], which is a modern system analysis tool being developed at Argonne National Laboratory for advanced nonLWR safety analyses. 
The system analysis code SAM aims to provide fast-running, whole-plant transient analysis capability with improved-fidelity for sodium-cooled fast reactors, lead-cooled fast reactors, and molten salt reactors or fluoride-cooled high-temperature reactors. SAM utilizes the Multi-physics Object-Oriented Simulation Environment (MOOSE) framework and its underlying meshing and finite-element library (libMesh) and linear and non-linear solvers (PETSc), to leverage the modern advanced software environments and numerical methods [12]. The MOOSE framework has been developed and maintained at Idaho National Laboratory under the NEAMS integration product line. The MOOSE framework is originally designed for fully coupled implicit multi-physics simulations based on finite element and the Jacobian-free Newton-Krylov (JFNK) methods in a quick and efficient manner. For loosely coupled systems or simulations involving external codes such as PROTEUS-NODAL, the MOOSE framework uses the operator splitting method to solve the coupled system of equations.

To apply the $\mathrm{P}_{1}$ solver to cylindrical geometry problems, a new $\mathrm{P}_{1}$ solver for $\mathrm{R}-\mathrm{Z}$ and $\mathrm{R}$ $\theta-Z$ geometries has been developed based on the variational nodal method. The $\mathrm{P}_{1}$ solver for cylindrical geometry problems has been verified against the DIF3D finite difference code [13]. In addition, transient solvers have been developed for all the $\mathrm{P}_{1}$ solvers using the transient fixed source problem (TFSP) formulation. The computational efficiency has also been improved significantly for transient problems by implementing the coarse mesh finite difference (CMFD) acceleration scheme into all the $\mathrm{P}_{1}$ solvers for Cartesian, triangular, hexagonal, and annular geometry problems. The steady state and transient $\mathrm{P}_{1}$ and $\mathrm{SP}_{3}$ solvers for MSR applications have been coupled with the SAM code under the MOOSE framework. The coupled system of PROTEUS-NODAL and SAM has been verified by comparing the MSFR benchmark solutions with the solutions found in the open literature and the PROTEUS-NODAL solutions obtained with the standalone thermal-hydraulics solver.

The purpose of this report is to document the new features and capabilities that have been implemented in the PROTEUS-NODAL code and the coupling procedure of PROTEUS-NODAL with SAM to analyze MSRs. This report is organized as follows. Section 2 presents the variational nodal $\mathrm{P}_{1}$ solver for cylindrical geometries and its verification test results. Section 3 discusses the transient analysis capability implemented in the $\mathrm{P}_{1}$ solvers of PROTEUS-NODAL. The solution methods for the coupled system of TFSP and precursor drift equations, the coarse mesh finite difference (CMFD) acceleration, the kinetics parameter evaluation method, and the thermal feedback are discussed. The verification test results against the $\mathrm{SP}_{3}$ solutions are also discussed. Section 4 describes the coupling scheme of PROTEUS-NODAL and SAM under the MOOSE framework for MSR applications. The developed MOOSE applications named "TreeFrog” and "TreeKangaroo" 
are discussed. Verification test results for the coupled system are also discussed by comparing the MSFR steady state solution with that obtained from a manually coupled PROTEUS-NODAL and ANSYS CFX [14] computational fluid dynamics (CFD) calculations and the MSFR transient analysis results with the open literature solutions and the PROTEUS-NODAL solutions obtained with the standalone thermal-hydraulics solver. Section 5 provides conclusions and future works. 


\section{Development of Variational Nodal $P_{1}$ Solver in $\mathbf{R}-\boldsymbol{\theta}-\mathbf{Z}$ Geometry}

The original implementation of the $\mathrm{P}_{1}$ and $\mathrm{SP}_{3}$ solvers of PROTEUS-NODAL has no solution option for cylindrical geometry problems. The $\mathrm{P}_{1}$ solver is applicable to Cartesian, hexagonal-Z, and triangular- $\mathrm{Z}$ geometries, and the $\mathrm{SP}_{3}$ solver is only for the hexagonal- $\mathrm{Z}$ geometry. As a result, the MSFR benchmark problem in cylindrical geometry has been analyzed using approximate hexagonal geometry models [9, 10]. In order to eliminate the unnecessary modeling approximation, a variation nodal $\mathrm{P}_{1}$ solver for $\mathrm{R}-\theta-\mathrm{Z}$ geometry has been developed. This section describes the variational nodal method in R- $\theta-\mathrm{Z}$ geometry and its implementation in PROTEUS-NODAL. The verification test results against the finite difference method (FDM) solutions of DIF3D [13] are also discussed along with the computational gain achieved with the cylindrical geometry model compared to the hexagonal geometry model of the MSFR.

The general variational nodal method for the diffusion theory is first described. This is followed by the discussion on the implemented $\mathrm{R}-\theta-\mathrm{Z}$ geometry solver, including the basis functions and the resulting response matrices. Then, the verification test results against the FDM solver of DIF3D are presented.

\subsection{Variational Nodal Method for Diffusion Theory}

In the variational nodal method for the diffusion theory, the functional for an internal node $v$ can be written as follows.

$$
\begin{aligned}
F_{v}\left[\phi_{g}, \chi_{g}\right]= & \int_{v} d V\left\{D_{g}(\boldsymbol{r}) \nabla \phi_{g}(\boldsymbol{r}) \cdot \nabla \phi_{g}(\boldsymbol{r})+\Sigma_{r, g}(\boldsymbol{r})\left(\phi_{g}(\boldsymbol{r})\right)^{2}-2 \phi_{g}(\boldsymbol{r}) S_{g}(\boldsymbol{r})\right\} \\
& +2 \sum_{\gamma} \int_{\Gamma_{\gamma}} d \Gamma_{\gamma} \int d \Omega\left(\boldsymbol{\Omega} \cdot \hat{\mathbf{n}}_{\gamma}\right) \phi_{g}(\boldsymbol{r}) \chi_{\gamma, g}(\boldsymbol{r}, \boldsymbol{\Omega}),
\end{aligned}
$$

where $V$ is the node volume, $\Gamma_{\gamma}$ is the area of surface $\gamma, g$ is the energy group index, $\phi_{g}(\boldsymbol{r})$

is the zeroth moment of the even parity flux (i.e., the scalar flux), $\chi_{\gamma, g}(\boldsymbol{r}, \mathbf{\Omega})$ is the first

moment of the odd parity flux on surface $\gamma$, and $S_{g}(\boldsymbol{r})$ is the isotropic source. Carrying out the angular integration in Eq. (2.1) yields

$$
\begin{aligned}
F_{v}\left[\phi_{g}, \chi_{g}\right] & =\int_{v} d V\left\{D_{g}(\boldsymbol{r}) \nabla \phi_{g}(\boldsymbol{r}) \cdot \nabla \phi_{g}(\boldsymbol{r})+\Sigma_{r, g}(\boldsymbol{r})\left(\phi_{g}(\boldsymbol{r})\right)^{2}-2 \phi_{g}(\boldsymbol{r}) S_{g}(\boldsymbol{r})\right\} \\
& +2 \sum_{\gamma} \int_{\gamma} d \Gamma_{\gamma} \phi_{g}(\boldsymbol{r}) j_{\gamma, g}(\boldsymbol{r}),
\end{aligned}
$$


where $j_{\gamma, g}(\boldsymbol{r})$ is the net surface current normal to the surface $\gamma$. By expanding the scalar flux and the surface current with orthogonal spatial basis functions, $f_{i}(\boldsymbol{r})$ and $h_{\gamma, j}(\boldsymbol{r})$, respectively, they can be expressed as

$$
\begin{aligned}
& \phi_{g}(\boldsymbol{r})=\sum_{i=1}^{N_{V}} f_{i}(\boldsymbol{r}) \zeta_{g, i}, \\
& j_{\gamma, g}(\boldsymbol{r})=\sum_{j=1}^{N_{s}} h_{\gamma, j}(\boldsymbol{r}) \chi_{\gamma, g, j},
\end{aligned}
$$

where $\zeta_{g, i}$ 's are the expansion coefficients of $N_{v}$ volumetric spatial basis functions $f_{i}(\boldsymbol{r})$ and $\chi_{\gamma, g, j}$ 's are the $N_{s}$ expansion coefficients of surface spatial basis functions $h_{\gamma, j}(\boldsymbol{r})$. The source is expanded with $f_{i}(\boldsymbol{r})$ and can be expressed as

$$
S_{g}(\boldsymbol{r})=\sum_{i} f_{i}(\boldsymbol{r}) S_{g, i} .
$$

Assuming constant cross sections in each node and using Eq. (2.3) and Eq. (2.4), Eq. (2.2) can be rewritten with the summation convention rule as

$$
\begin{aligned}
F_{v}\left[\phi_{g}, \chi_{g}\right] & =D_{g} \int_{v} d V \nabla_{\alpha} f_{i}(\boldsymbol{r}) \zeta_{g, i} \nabla_{\alpha} f_{j}(\boldsymbol{r}) \zeta_{g, j}+\Sigma_{r, g} \int_{v} d V f_{i}(\boldsymbol{r}) \zeta_{g, i} f_{j}(\boldsymbol{r}) \zeta_{g, j} \\
& -2 \int_{v} d V f_{i}(\boldsymbol{r}) \zeta_{g, i} f_{j}(\boldsymbol{r}) \zeta_{g, j} s_{g, j}+2 \sum_{\gamma} \int_{\gamma} d \Gamma_{\gamma} f_{i}(\boldsymbol{r}) \zeta_{g, i} h_{\gamma, j}(\boldsymbol{r}) \chi_{\gamma, g, j},
\end{aligned}
$$

where $\alpha$ sums over $x, y$, and $z$ for Cartesian, hexagonal, and triangular-z geometries and over $r, \theta$, and $z$ for cylindrical geometries. Using matrix and vector notations, Eq. (2.5) can be expressed as

$$
F_{v}\left[\phi_{g}, \chi_{g}\right]=\zeta_{g}^{T}\left(D_{g} \overline{\mathbf{P}}+\Sigma_{r, g} \overline{\mathbf{F}}\right) \zeta_{g}-2 \boldsymbol{\zeta}_{g}^{T} \overline{\mathbf{F}} \mathbf{s}_{g}+2 \sum_{\gamma} \zeta_{g}^{T} \overline{\mathbf{M}}_{\gamma} \boldsymbol{\chi}_{\gamma, g}
$$

where $\zeta_{g}=\left[\zeta_{g, 1}, \ldots, \zeta_{g, N_{v}}\right]^{T}, \chi_{\gamma, g}=\left[\chi_{\gamma, g, 1}, \ldots, \chi_{\gamma, g, N_{s}}\right]^{T}$, and $\mathbf{s}_{g}=\left[s_{g, 1}, \ldots, s_{g, N_{v}}\right]^{T}$. The elements at the $i$-th row and the $j$-th column of the matrices $\overline{\mathbf{P}}, \overline{\mathbf{F}}$, and $\overline{\mathbf{M}}_{\gamma}$ are given by

$$
\begin{aligned}
& \overline{\mathbf{P}}_{i j}=\sum_{\alpha} \int_{v} d V \nabla_{\alpha} f_{i}(\boldsymbol{r}) \nabla_{\alpha} f_{j}(\boldsymbol{r}), \\
& \overline{\mathbf{F}}_{i j}=\int_{v} d V f_{i}(\boldsymbol{r}) f_{j}(\boldsymbol{r})=V_{v} \delta_{i j}, \\
& \overline{\mathbf{M}}_{\gamma, i j}=\int_{\gamma} d \Gamma_{\gamma} f_{i}(\boldsymbol{r}) h_{\gamma, j}(\boldsymbol{r}) .
\end{aligned}
$$


The spatial basis functions are orthogonalized with the Gram-Schmidt orthogonalization procedure so that $\overline{\mathbf{F}}_{i j}=V_{v} \delta_{i j}$ [15]. As a result, the first element of the coefficient vector of the scalar flux becomes the node-averaged scalar flux yielding

$$
\zeta_{g, 1}=\frac{1}{V_{v}} \int_{v} d V \phi_{g}(\boldsymbol{r}) f_{1}(\boldsymbol{r})=\frac{1}{V_{v}} \int_{v} d V \phi_{g}(\boldsymbol{r})=\bar{\phi}_{g} .
$$

Requiring the reduced functional in Eq. (2.6) be stationary with respect to the variation of the scalar flux results in

$$
\zeta_{g}=\overline{\mathbf{A}}_{g}^{-1}\left(\overline{\mathbf{F}} \mathbf{s}_{g}-\sum_{\gamma} \overline{\mathbf{M}}_{\gamma, g} \boldsymbol{\chi}_{\gamma, g}\right)
$$

where $\overline{\mathbf{A}}_{g}=D_{g} \overline{\mathbf{P}}+\sum_{r, g} \overline{\mathbf{F}}$. In order to use a nodal response matrix approach, an additional relation that couples interfacial scalar fluxes (or interfacial partial currents) and node average scalar fluxes between nodes is needed. This relation can be obtained by requiring the surface current to be continuous across each interface. To represent the surface scalar flux $\varphi_{\gamma, g}(\boldsymbol{r})=\phi_{g}\left(\boldsymbol{r} \in \Gamma_{\gamma}\right)$ in terms of the node average scalar flux, $\varphi_{\gamma, g}(\boldsymbol{r})$ can be projected onto each surface basis function as

$$
\int_{\gamma} d \Gamma_{\gamma} h_{\gamma, i}(\boldsymbol{r}) \varphi_{\gamma, g}(\boldsymbol{r})=\int_{\gamma} d \Gamma_{\gamma} h_{\gamma, i}(\boldsymbol{r}) \phi_{g}\left(\boldsymbol{r} \in \Gamma_{\gamma}\right) .
$$

By orthogonalizing the surface basis functions such that $\int_{\gamma} d \Gamma_{\gamma} h_{\gamma, i}(\boldsymbol{r}) h_{\gamma, j}(\boldsymbol{r})=\Gamma_{\gamma} \delta_{i j}$ and by expanding the surface scalar flux with the orthogonalized surface basis functions as $\varphi_{\gamma, g}(\boldsymbol{r})=\sum_{j} h_{\gamma, j}(\boldsymbol{r}) \varphi_{\gamma, g, j}$, the following relation is obtained.

$$
\Gamma_{\gamma} \boldsymbol{\varphi}_{\gamma, g}=\sum_{j} \int_{\gamma} d \Gamma_{\gamma} h_{\gamma, i}(\boldsymbol{r}) f_{j}(\boldsymbol{r}) \zeta_{g, j}=\overline{\mathbf{M}}_{\gamma}^{T} \zeta_{g},
$$

where $\boldsymbol{\varphi}_{\gamma, g}=\left[\varphi_{\gamma, g, 1}, \ldots, \varphi_{\gamma, g, N_{s}}\right]^{T}$ is the coefficient vector of the surface flux. Because of the orthogonality of the surface basis functions, the first element of the coefficient vector of the surface current becomes the surface-averaged current as

$$
\chi_{\gamma, g, 1}=\frac{1}{\Gamma_{\gamma}} \int_{\gamma} d \Gamma_{\gamma} j_{\gamma, g}(\boldsymbol{r}) h_{1}(\boldsymbol{r})=\frac{1}{\Gamma_{\gamma}} \int_{\gamma} d \Gamma_{\gamma} j_{\gamma, g}(\boldsymbol{r})=\bar{j}_{\gamma, g} .
$$

By inserting Eq. (2.9) into Eq. (2.11), the surface flux is expressed as

$$
\boldsymbol{\varphi}_{\gamma, g}=\tilde{\mathbf{M}}_{\gamma}^{T} \overline{\mathbf{A}}_{g}^{-1} \overline{\mathbf{F}}_{g}-\tilde{\mathbf{M}}_{\gamma}^{T} \overline{\mathbf{A}}_{g}^{-1} \sum_{\gamma^{\prime}} \overline{\mathbf{M}}_{\gamma^{\prime}} \boldsymbol{\chi}_{\gamma^{\prime}, g},
$$


where $\tilde{\mathbf{M}}_{\gamma}^{T}=\overline{\mathbf{M}}_{\gamma}^{T} / \Gamma_{\gamma}$. In the diffusion approximation, $\boldsymbol{\varphi}_{\gamma, g}=2\left(\chi_{\gamma, g}^{+}+\chi_{\gamma, g}^{-}\right)$where $\boldsymbol{\chi}_{\gamma, g}^{ \pm}=\left[\chi_{\gamma, g, 1}^{ \pm}, \ldots, \chi_{\gamma, g, N_{s}}^{ \pm}\right]^{T}$ are the surface partial currents. Therefore, Eq. (2.13) can be rewritten in terms of partial currents as

$$
\boldsymbol{\chi}_{\gamma, g}^{+}+\boldsymbol{\chi}_{\gamma, g}^{-}=\frac{1}{2} \tilde{\mathbf{M}}_{\gamma}^{T} \overline{\mathbf{A}}_{g}^{-1} \overline{\mathbf{F}} \mathbf{s}_{g}-\frac{1}{2} \tilde{\mathbf{M}}_{\gamma}^{T} \overline{\mathbf{A}}_{g}^{-1} \sum_{\gamma^{\prime}} \overline{\mathbf{M}}_{\gamma^{\prime}}\left(\chi_{\gamma^{\prime}, g}^{+}-\chi_{\gamma^{\prime}, g}^{-}\right) .
$$

Eq. (2.9) and Eq. (2.14) can be rewritten in the following response matrix equations:

$$
\begin{gathered}
\zeta_{g}=\overline{\mathbf{H}}_{g} \mathbf{s}_{g}-\overline{\mathbf{C}}_{g}\left(\mathbf{J}_{g}^{+}-\mathbf{J}_{g}^{-}\right), \\
\mathbf{J}_{g}^{+}=\overline{\mathbf{B}}_{g} \mathbf{s}_{g}+\overline{\mathbf{R}}_{g} \mathbf{J}_{g}^{-},
\end{gathered}
$$

where

$$
\begin{aligned}
& \mathbf{J}_{g}^{ \pm}=\left[\boldsymbol{\chi}_{1, g}^{ \pm}, \cdots, \boldsymbol{\chi}_{N_{\gamma}, g}^{ \pm}\right]^{T}, \\
& \overline{\mathbf{H}}_{g}=\overline{\mathbf{A}}_{g}^{-1} \overline{\mathbf{F}}, \\
& \overline{\mathbf{B}}_{g}=\left(\overline{\mathbf{G}}_{g}+\mathbf{I}\right)^{-1} \frac{1}{2} \tilde{\mathbf{M}}^{T} \overline{\mathbf{A}}_{g}^{-1} \overline{\mathbf{F}}, \\
& \overline{\mathbf{R}}_{g}=\left(\overline{\mathbf{G}}_{g}+\mathbf{I}\right)^{-1}\left(\overline{\mathbf{G}}_{g}-\mathbf{I}\right) .
\end{aligned}
$$

The block matrices $\overline{\mathbf{G}}_{g}, \overline{\mathbf{C}}_{g}$, and $\tilde{\mathbf{M}}$ in Eq. (2.17) are defined as

$$
\overline{\mathbf{G}}_{g}=\left[\begin{array}{ccc}
\mathbf{G}_{11, g} & \cdots & \mathbf{G}_{1 N_{\gamma}, g} \\
\vdots & \ddots & \vdots \\
\mathbf{G}_{N_{\gamma} 1, g} & \cdots & \mathbf{G}_{N_{\gamma} N_{\gamma}, g}
\end{array}\right], \quad \overline{\mathbf{C}}_{g}=\left[\mathbf{C}_{1, g}, \ldots, \mathbf{C}_{N_{\gamma}, g}\right], \quad \tilde{\mathbf{M}}=\left[\overline{\mathbf{M}}_{1} / \Gamma_{1}, \cdots, \overline{\mathbf{M}}_{N_{\gamma}} / \Gamma_{N_{\gamma}}\right]
$$

where $\mathbf{G}_{\gamma \gamma^{\prime}, g}=\frac{1}{2} \tilde{\mathbf{M}}_{\gamma}^{T} \overline{\mathbf{A}}_{g}^{-1} \overline{\mathbf{M}}_{\gamma^{\prime}}, \mathbf{C}_{\gamma, g}=\overline{\mathbf{A}}_{g}^{-1} \overline{\mathbf{M}}_{\gamma}$, and $N_{\gamma}$ is the number of surfaces.

For a given source, Eq. (2.16) is solved iteratively. For each node, by using the incoming surface partial currents as an input, the outgoing surface partial currents are updated. Then, the outgoing surface partial currents are used as the incoming surface partial currents for adjacent nodes using the Red-Black sweeping scheme. Once the currents converge, nodeaverage scalar fluxes are updated. 


\subsection{Variational Nodal $P_{1}$ Solver in $R-\vartheta-Z$ Geometry}

In this section, the orthogonal basis functions $f_{i}(\boldsymbol{r})$ and $h_{\gamma, j}(\boldsymbol{r})$ for R- $\theta$-Z geometry are first derived. Then the procedure to generate the response matrices based on these basis functions to be compatible for use with the CMFD acceleration scheme is discussed. The method to treat the periodic boundary condition to the $\theta$ direction is also discussed.

\subsubsection{Generation of Basis Functions}

The basis functions for the R- $\theta-\mathrm{Z}$ geometry are polynomials with a user-specified order expressed with monomials as

$$
\begin{aligned}
& f_{i}(\boldsymbol{r})=\sum_{p} \sum_{q} \sum_{w} C_{i}^{p q w} r^{p} \theta^{q} z^{w}, \\
& h_{\gamma, j}(\boldsymbol{r})=\sum_{p} \sum_{q} C_{\gamma, j}^{p q} \alpha^{p} \beta^{q}, \alpha, \beta=\text { coordinate variables on surface } \gamma .
\end{aligned}
$$

The coefficients $C_{i}^{p q w}$ and $C_{\gamma, j}^{p q}$ are determined by the Gram-Schmidt orthogonalization procedure. In Cartesian, hexagonal, and triangular geometries, these coefficients are the same for all the nodes because all the nodes have the translational symmetry with arbitrary scaling. However, there is no translational symmetry in cylindrical geometry, and thus the volumetric basis functions and surface basis functions in R- $\theta$ and $\mathrm{R}-\mathrm{Z}$ surfaces are unique and generated for each radial node.

For outer nodes of annular sector shape, the sequence of $p, q$, and $w$ and the coefficients in Eq. (2.19) are determined in the same way as the other geometry types. However, the central nodes of sector shape need a special care. At the center, the volumetric basis functions must not depend on $\theta$ and their derivatives with respect to $r$ should be zero. Thus, the following constraints should be imposed to the volumetric basis functions for central nodes.

$$
\begin{gathered}
\left.\frac{\partial f_{i}(r, \theta, z)}{\partial r}\right|_{r=0}=0, \\
f_{i}(r, \theta, z)=g(0, z) .
\end{gathered}
$$

These are equivalent to the following constraints on the monomials of $r^{p} \theta^{q} z^{w}$ :

1. Any monomials with $p=1$ are not allowed.

2. For $p=0$, only $q=0$ is allowed.

Thus, the monomials allowed for the volumetric basis functions of the central nodes become 


$$
1, z, r^{2}, z^{2}, r^{3}, r^{2} \theta, r^{2} z, z^{3}, r^{4}, r^{3} \theta, r^{3} z, r^{2} \theta^{2}, r^{2} z^{2}, r^{2} \theta z, z^{4}, \cdots
$$

The same constraints are applied to the relevant surface basis functions. The allowed monomials for the surface basis functions are

$$
\begin{aligned}
& \text { Surface 1, 2: 1, } \theta, z, \theta^{2}, \theta z, z^{2}, \theta^{3}, \theta^{2} z, \theta z^{2}, z^{3}, \cdots \\
& \text { Surface 3, 4: 1, } z, r^{2}, z^{2}, r^{3}, r^{2} z, z^{3}, r^{4}, r^{3} z, r^{2} z^{2}, z^{4}, \cdots \\
& \text { Surface 5, 6: } 1, r^{2}, r^{3}, r^{2} \theta, r^{4}, r^{3} \theta, r^{2} \theta^{2}, \cdots,
\end{aligned}
$$

with the surface numbering scheme present in Table 2.1. Note that a central node has only five surfaces from Surface 2 to Surface 6.

Table 2.1. Surface Numbering Scheme in the R- $\theta-Z$ Geometry

\begin{tabular}{|l|l|l|l|l|}
\hline \multirow{2}{*}{ Surface Number } & \multicolumn{4}{l|}{ Surface Type per Geometry Type } \\
\cline { 2 - 5 } & $R-\theta-Z$ & $R-\theta$ & $R-Z$ & $R$ \\
\hline 1 & $\theta-z @-r$ & $\theta @-r$ & $z @-r$ & @ $-r$ \\
\hline 2 & $\theta-z @+r$ & $\theta @+r$ & $z @+r$ & @ $+r$ \\
\hline 3 & $r-z @-\theta$ & $r @-\theta$ & $r @-z$ & \\
\hline 4 & $r-z @+\theta$ & $r @+\theta$ & $r @+z$ & \\
\hline 5 & $r-\theta @-z$ & & & \\
\hline 6 & $r-\theta @+z$ & & & \\
\hline
\end{tabular}

*Variables@ @ $\gamma$ represents a surface depending on "variables” at a constant $\gamma$ with "+” for the larger value and " -“ for the smaller value of variable $\gamma$.

To keep the same number of moments for the volumetric and surface basis functions for all the nodes, the monomials in Eq. (2.22) for the central nodes can include higher order terms than the user specification. That is, the basis functions for the surfaces 3 to 6 that are constrained by Eq. (2.20) will contain monomials of higher orders.

Using the selected set of monomials, the volumetric and surface basis functions are generated with the Gram-Schmidt orthogonalization procedure. The normalization condition for the volumetric basis functions is given by

$$
\overline{\mathbf{F}}_{i j}^{\text {code }}=\int_{r_{1}}^{r_{2}} d r r \int_{-1 / 2}^{+1 / 2} d \theta \int_{-1 / 2}^{+1 / 2} d z \tilde{f}_{i}(\boldsymbol{r}) \tilde{f}_{j}(\boldsymbol{r})=\delta_{i j},
$$

where $\tilde{f}_{i}$ 's are different from $f_{i}$ 's in Eq. (2.3) that are normalized to satisfy Eq. (2.8). The superscript code differentiates $\overline{\mathbf{F}}^{\text {code }}$ from $\overline{\mathbf{F}}$ in Eq. (2.7). The surface basis functions for R$\theta-\mathrm{Z}$ geometry are also orthonormalized as 


$$
\overline{\mathbf{I}}_{\gamma, i j}^{\text {code }}=\left\{\begin{array}{l}
r_{\gamma} \int_{-1 / 2}^{+1 / 2} d \theta \int_{-1 / 2}^{+1 / 2} d z \tilde{h}_{\gamma, i}(\boldsymbol{r}) \tilde{h}_{\gamma, j}(\boldsymbol{r})=\delta_{i j} \text { for } \gamma=1,2, \\
\int_{r_{1}}^{r_{2}} d r \int_{-1 / 2}^{+1 / 2} d z \tilde{h}_{\gamma, i}(\boldsymbol{r}) \tilde{h}_{\gamma, j}(\boldsymbol{r})=\delta_{i j} \text { for } \gamma=3,4, \\
\int_{r_{1}}^{r_{2}} d r \int_{-1 / 2}^{+1 / 2} r d \theta \tilde{h}_{\gamma, i}(\boldsymbol{r}) \tilde{h}_{\gamma, j}(\boldsymbol{r})=\delta_{i j} \text { for } \gamma=5,6 .
\end{array}\right.
$$

For the other two-dimensional (2D) and one-dimensional (1D) geometries in Table 2.1, the relevant normalization conditions in Eq. (2.24) are used according to their surface types. Again, $\tilde{h}_{\gamma, i}$ 's in Eq. (2.24) are different from $h_{\gamma, i}$ 's in Eq. (2.3) that are normalized to satisfy the condition $\int_{\gamma} d \Gamma_{\gamma} h_{\gamma, i}(\boldsymbol{r}) h_{\gamma, j}(\boldsymbol{r})=\Gamma_{\gamma} \delta_{i j}$.

The Gram-Schmidt orthogonalization procedure does not produce accurate results for large $r$ values because of increasing round off errors. Therefore, the quadruple precision was used in the orthogonalization procedure by defining separate routines that deal with 16-byte real variables.

\subsubsection{Generation of Response Matrices}

Once the basis functions are generated, the response matrices can be generated using Eq. (2.7), Eq. (2.17), and Eq. (2.18). In order to comply with the CMFD acceleration, it is better to carry the scalar fluxes and surface currents that satisfy the normalization conditions in Eq. (2.8) and Eq. (2.12). For this, the $\overline{\mathbf{P}}^{\text {code }}$ and $\overline{\mathbf{M}}_{\gamma}^{\text {code }}$ matrices resulting from the basis functions generated with Eq. (2.23) and Eq. (2.24), are multiplied by the scaling factors in Table 2.2 to satisfy the normalization conditions in Eq. (2.8) and Eq. (2.12). Using these scaled $\overline{\mathbf{F}}, \overline{\mathbf{P}}$, and $\overline{\mathbf{M}}_{\gamma}$ matrices, the final response matrices are generated using Eq. (2.17) and Eq. (2.18). 
Table 2.2. Geometry Dependent Matrix Scaling Factors

\begin{tabular}{|l|l|l|l|l|}
\hline & $R-\theta-Z$ & $R-\theta$ & $R-Z$ & $R$ \\
\hline$\overline{\mathbf{F}}$ & $\bar{r}_{m} \Delta r_{m} \Delta \theta_{m} \Delta z_{m}$ & $\bar{r}_{m} \Delta r_{m} \Delta \theta_{m}$ & $2 \pi \bar{r}_{m} \Delta r_{m} \Delta z_{m}$ & $2 \pi \bar{r}_{m} \Delta r_{m}$ \\
\hline$\overline{\mathbf{P}}^{r}$ & $\bar{r}_{m} \Delta r_{m} \Delta \theta_{m} \Delta z_{m}$ & $\bar{r}_{m} \Delta r_{m} \Delta \theta_{m}$ & $2 \pi \bar{r}_{m} \Delta r_{m} \Delta z_{m}$ & $2 \pi \bar{r}_{m} \Delta r_{m}$ \\
\hline$\overline{\mathbf{P}}^{\theta}$ & $\bar{r}_{m} \Delta r_{m} \Delta z_{m} / \Delta \theta_{m}$ & $\bar{r}_{m} \Delta r_{m} / \Delta \theta_{m}$ & & \\
\hline$\overline{\mathbf{P}}^{z}$ & $\bar{r}_{m} \Delta r_{m} \Delta \theta_{m} / \Delta z_{m}$ & & $2 \pi \bar{r}_{m} \Delta r_{m} / \Delta z_{m}$ & \\
\hline$\overline{\mathbf{M}}_{1,2}$ & $\sqrt{\bar{r}_{1,2} \bar{r}_{m} \Delta r_{m}} \Delta \theta_{m} \Delta z_{m}$ & $\sqrt{\bar{r}_{1,2} \bar{r}_{m} \Delta r_{m}} \Delta \theta_{m}$ & $2 \pi \sqrt{\bar{r}_{1,2} \bar{r}_{m} \Delta r_{m}} \Delta z_{m}$ & $2 \pi \sqrt{\bar{r}_{1,2} \bar{r}_{m} \Delta r_{m}}$ \\
\hline$\overline{\mathbf{M}}_{3,4}$ & $\sqrt{\bar{r}_{m}} \Delta r_{m} \Delta z_{m}$ & $\sqrt{\bar{r}_{m}} \Delta r_{m}$ & $2 \pi \bar{r}_{m} \Delta r_{m}$ & \\
\hline$\overline{\mathbf{M}}_{5,6}$ & $\bar{r}_{m} \Delta r_{m} \Delta \theta_{m}$ & & & \\
\hline
\end{tabular}

$* \mathrm{~m}$ = node index, $r_{1,2}$ = radii of the inner and the outer boundary surfaces, $\bar{r}_{m}=\left(r_{1}+r_{2}\right) / 2$.

\subsubsection{Boundary Conditions in $\vartheta$ Direction}

The allowed boundary conditions in the $\theta$ direction are the periodic, vacuum and extrapolated ones. The periodic boundary condition was realized by assigning the same surface numbers to the surfaces at the both ends in the $\theta$ direction. In this way, the interface partial currents can be mapped between the nodes at the both ends in the $\theta$ direction. Even though the reflective boundary condition can also be realized with a simple mapping of interface partial currents for the diffusion approximation, it is not the case when the transport theory is considered. Thus, to avoid the loss of generality, the reflective boundary condition to the $\theta$ direction is not allowed.

\subsection{Verification Tests of Variational Nodal $P_{1}$ Solver for $R-\vartheta-Z$ Geometry}

Using the MSFR benchmark problem of which description is given in Appendix A, the developed variational nodal $\mathrm{P}_{1}$ solver for $\mathrm{R}-\theta-\mathrm{Z}$ geometry problems was verified against the FDM solver of the DIF3D code. First, the $\mathrm{P}_{1}$ solver of PROTEUS-NODAL for R-Z geometry was verified by solving the MSFR benchmark problem for a steady state without the dependency on the azimuthal direction. In the second problem, the $\mathrm{P}_{1}$ solver for $\mathrm{R}-\theta-\mathrm{Z}$ geometry was verified by solving a modified MSFR problem with three control rods located $120^{\circ}$ apart from each other. The computational gain of using the cylindrical geometry compared to the hexagonal geometry was also examined by solving the same problems with the $\mathrm{P}_{1}$ solver in hexagonal geometry.

\subsubsection{R-Z Geometry Problem}

The steady state MSFR benchmark problem was solved using the R-Z geometry shown in Fig. 2.1. The radial configuration at the core level is shown in Fig. 2.2. The left geometry of Fig. 2.2 was used in the $\mathrm{P}_{1}$ solver for R-Z geometry and DIF3D-FDM. The radial node size 
was $\sim 15 \mathrm{~cm}$, and the axial node size was $10 \mathrm{~cm}$ in the upper and lower reflectors and 11.275 $\mathrm{cm}$ for the core. Two DIF3D-FDM calculations were performed: one with $1 \mathrm{~cm} \times 1 \mathrm{~cm}$ mesh size and the other one with $2 \mathrm{~cm} \times 2 \mathrm{~cm}$ mesh size. The reference solution was obtained with the Richardson extrapolation of the two results.

The approximate hexagonal geometry problems in the right figure of Fig. 2.2 and in Fig. 2.3 were also solved using the $\mathrm{P}_{1}$ solver in hexagonal geometry. In these models, the pitch was determined to preserve the volume of the fuel region at the core center with a given number of rings. The rest regions were constructed to preserve the volume of each region as close as possible. The model of $9 \mathrm{~cm}$-pitch hexagons was prepared as the smallest hexagon case in order to estimate the computational gain of the $\mathrm{R}-\mathrm{Z}$ model relative to the hexagonal model of comparable accuracy. In all the calculations, a $60^{\circ}$ periodic boundary condition was used.

The ${ }^{233} \mathrm{U}$-started composition was used in the calculation. The $\mathrm{MC}^{2}-3$ and the TWODANT codes were used to generate an ISOTXS file with the 33-group structure. The same ISOTXS file was used both in DIF3D-FDM and PROTEUS-NODAL.

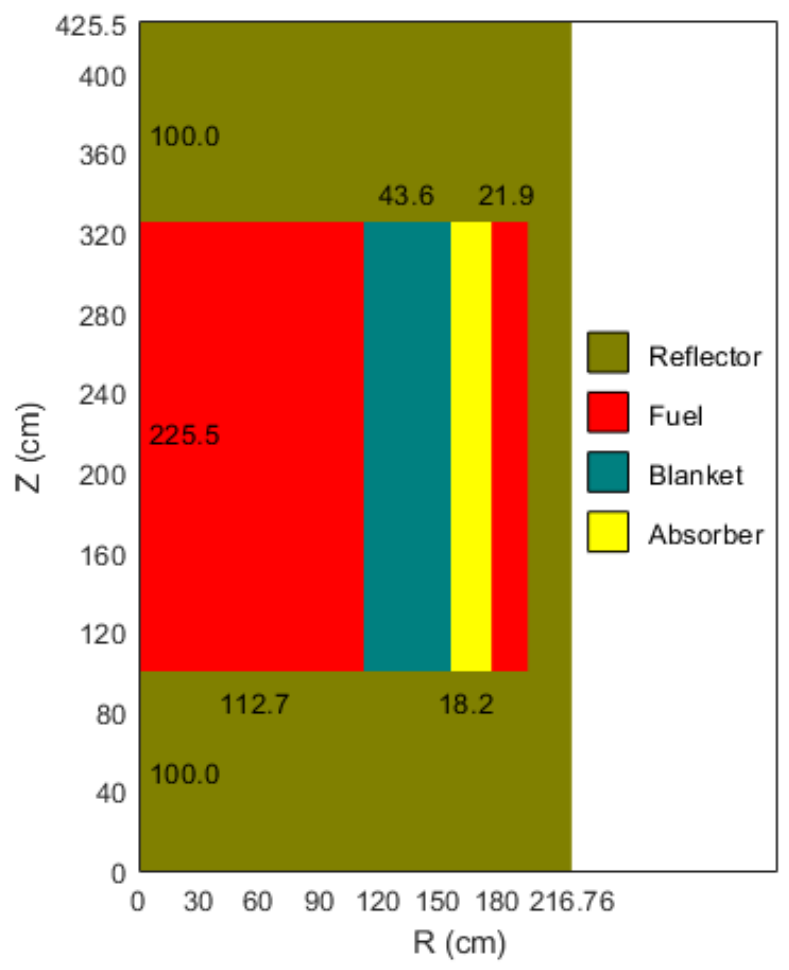

Fig. 2.1. Simplified R-Z geometry of MSFR. 

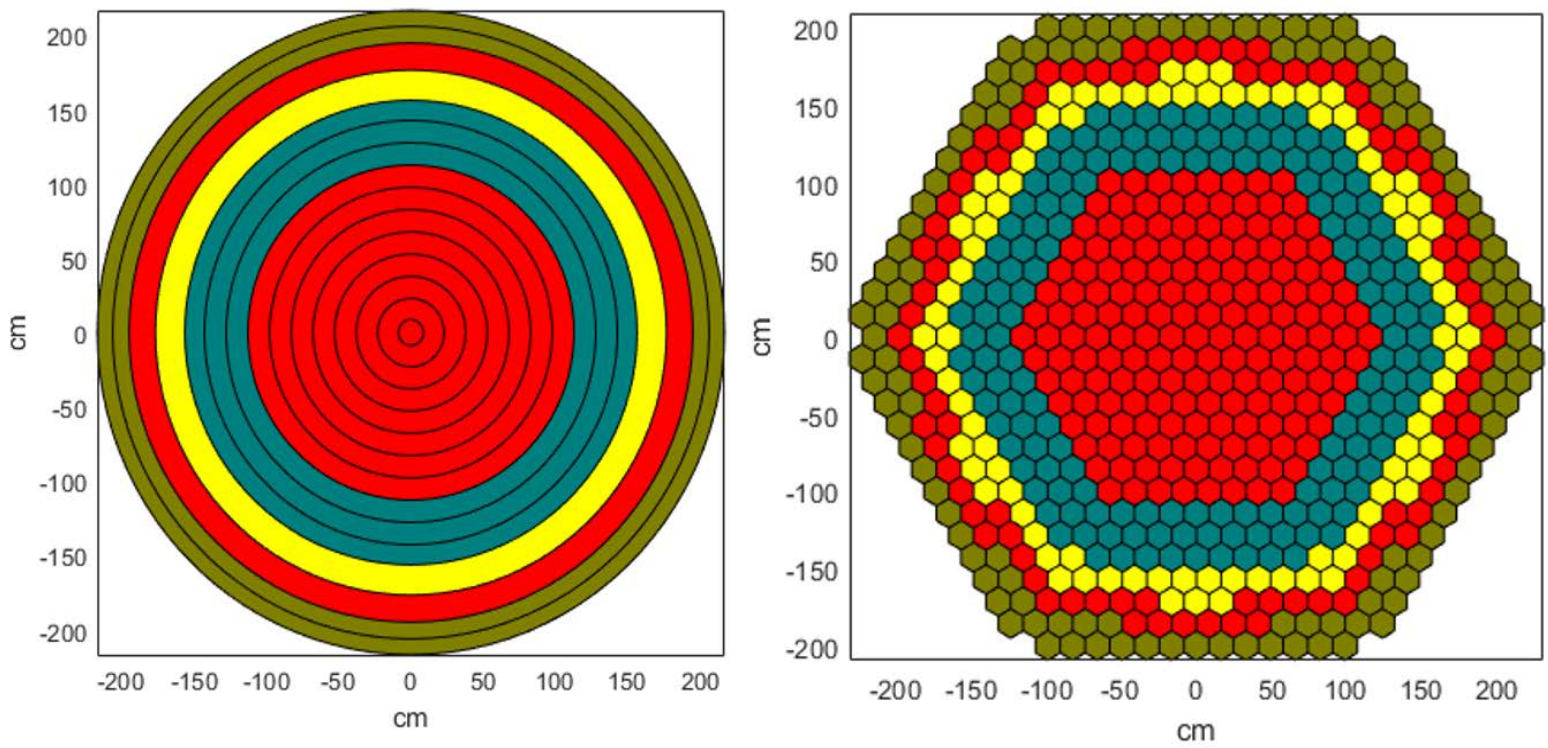

Fig. 2.2. MSFR core models in original cylindrical geometry (left) and in approximate hexagonal geometry of 8 rings of hexagons of $16.5 \mathrm{~cm}$ pitch (right).
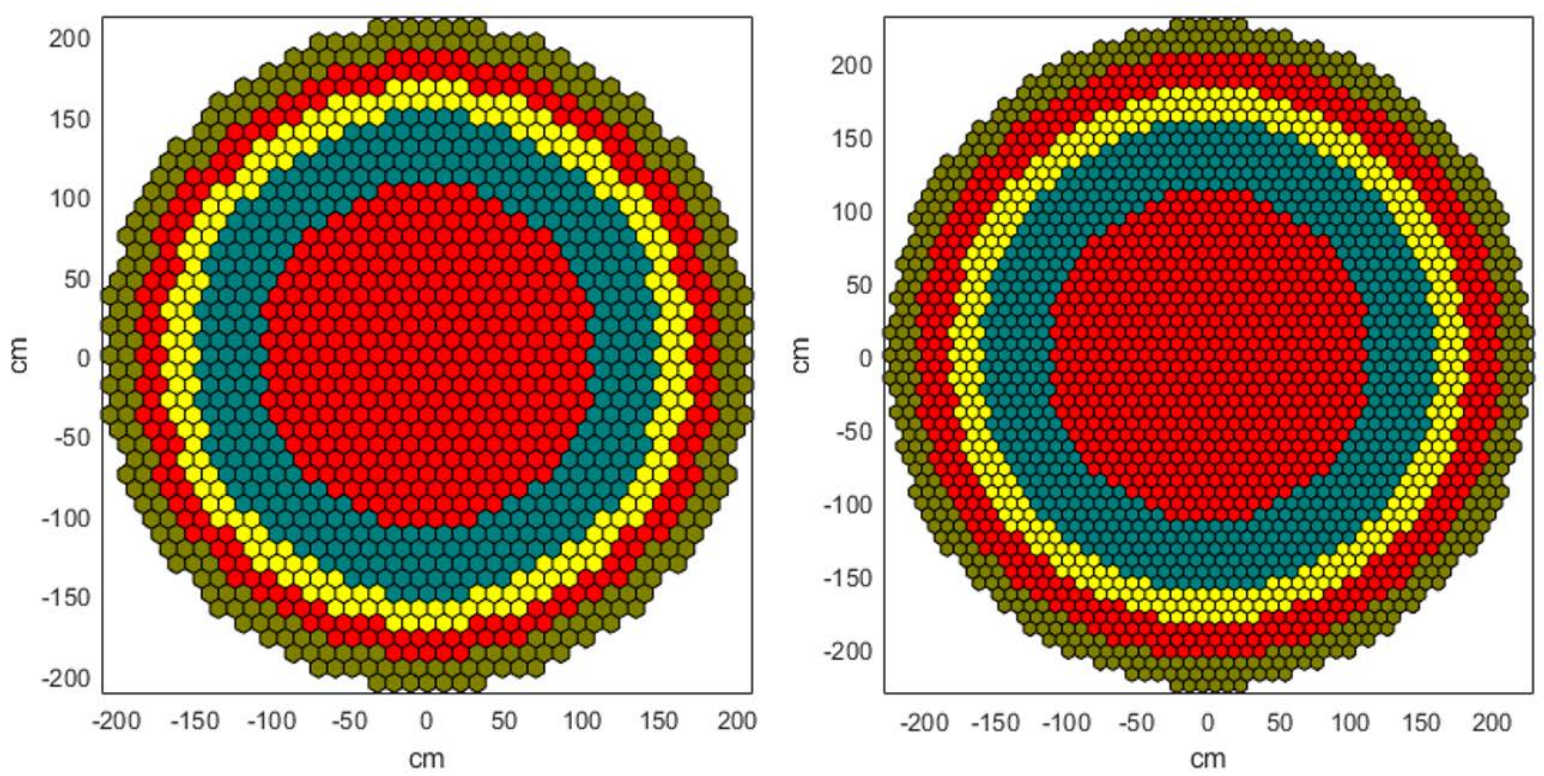

Fig. 2.3. Approximated hexagonal geometry core models of MSFR with 12 rings of hexagons of $11.3 \mathrm{~cm}$ (left) and 15 rings of hexagons of $9.0 \mathrm{~cm}$ pitch (right). 
The zero incoming current boundary condition ( $\alpha=0.5$ in $\alpha=J / \phi)$ was used for all directions: $\mathrm{A}=0.5$ in the type 05 card in NIP3 for the DIF3D calculation and the VACUUM boundary condition for the PROTEUS-NODAL calculation. For the convergence criteria, $10^{-}$ ${ }^{7}$ and $10^{-5}$ were used for the eigenvalue and the fission source convergence, respectively. No acceleration techniques for fission source convergence were applied both in DIF3D-FDM and PROTEUS-NODAL for consistent comparison.

The calculation results are summarized in Table 2.3. The $\% \Delta P$ column in the table means the relative error in the average power densities in the central core region nodes of $\sim 15 \mathrm{~cm} \times 11.275$ size. To calculate the power distribution error in the hexagonal geometry, the power density at the center of each hexagon was regarded as the point quantity and the radial average values in a ring of interest were obtained with the weighting function $r$ in the integration range of the inner and the outer radial boundaries of the ring of interest. Thus, the error represents the discretization error of approximating a ring with hexagons.

Table 2.3. Eigenvalue and Power Comparison Results of Unrodded Case

\begin{tabular}{|l|l|l|l|l|}
\hline & \multicolumn{1}{|c|}{ K-eff (error) } & $\begin{array}{c}\% \Delta P^{\mathrm{a}} \\
\text { RMS, MAX }\end{array}$ & $\begin{array}{c}\text { Time } \\
(\mathrm{s})\end{array}$ & $\begin{array}{l}\text { \# of Outer } \\
\text { Iterations }\end{array}$ \\
\hline FDM $(2 \mathrm{~cm} \times 2 \mathrm{~cm})^{\mathrm{b}}$ & $0.98375(+8 \mathrm{pcm})$ & $0.38,1.69$ & 117 & \\
\hline FDM $(1 \mathrm{~cm} \times 1 \mathrm{~cm})$ & $0.98369(+2 \mathrm{pcm})$ & $0.026,0.097$ & 824 & 97 \\
\hline Extrapolated $($ Reference $)$ & 0.98367 & - & - & \\
\hline R-Z Nodal $(\sim 15 \mathrm{~cm})$ & $0.98366(-1 \mathrm{pcm})$ & $0.0087,0.051$ & 1.5 & 49 \\
\hline Hexagonal Nodal $(9.0 \mathrm{~cm})$ & $0.98365(-2 \mathrm{pcm})$ & $0.55,1.47$ & 161.8 & 44 \\
\hline Hexagonal Nodal $(11.3 \mathrm{~cm})$ & $0.98339(-28 \mathrm{pcm})$ & $1.12,3.16$ & 91.0 & 44 \\
\hline Hexagonal Nodal $(16.5 \mathrm{~cm})$ & $0.98278(-89 \mathrm{pcm})$ & $2.59,7.31$ & 40.6 & 44 \\
\hline
\end{tabular}

* All nodal calculations used $10 \mathrm{~cm}$ and $11.275 \mathrm{~cm}$ axial node sizes for the axial reflectors and core regions and the sixth and linear expansion orders for volumetric and surface basis functions, respectively.

${ }^{a}$ Averaged over each ring in the fuel region with $11.275 \mathrm{~cm}$ in the axial direction and 15 $\mathrm{cm}$ in the radial direction.

${ }^{\mathrm{b}} \Delta r \times \Delta z$

It can be seen that the eigenvalue and power distribution results of the $\mathrm{P}_{1}$ solver for $\mathrm{R}-\mathrm{Z}$ geometry agree very well with the reference solutions obtained by Richardson extrapolation of two DIF3D-FDM solutions. When the hexagonal geometry model was used to approximate the cylindrical geometry, a comparable accuracy to the R-Z model solution was obtained with a $9 \mathrm{~cm}$ hexagonal pitch. The eigenvalue of this hexagonal geometry model agrees very well with the reference value, but the power distribution error is comparable to the DIF3D-FDM solution of $2 \mathrm{~cm}$ meshes. It can be seen that for a comparable computational 
accuracy, the R-Z model reduces the computational time about 100 times relative to the hexagonal geometry model.

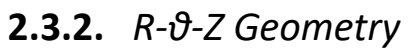

To verify the $\mathrm{P}_{1}$ solver for $\mathrm{R}-\theta-\mathrm{Z}$ geometry, three virtual control rods were introduced $120^{\circ}$ apart from each other as shown in Fig. 2.4 and Fig. 2.5. The control rods are about $\sim 10$ $\mathrm{cm}$ wide, and the volumes are the same in the R- $\theta-\mathrm{Z}$ geometry and the hexagonal geometry. To introduce the control rods, the third and the fourth rings in the left figure of Fig. 2.2 were merged and subdivided into three rings where the control rods were inserted in the middle ring. The radial node sizes of all the other nodes and the axial node sizes of all nodes were kept the same as in the unrodded case. Two calculations were performed with the R- $\theta-\mathrm{Z}$ geometry by varying node sizes in the azimuthal direction. One used $13.3^{\circ}$ for the nodes containing a control rod and $17.8^{\circ}$ for other nodes, and the other used the half-sized nodes. For the hexagonal geometry, only the $9 \mathrm{~cm}$-pitch hexagonal model was used in the calculation. In all the PROTEUS-NODAL calculations, the $120^{\circ}$ periodic boundary condition was used. For the DIF3D-FDM calculations, radial and axial meshes were kept the same as in the unrodded case and $120^{\circ}$ was divided into 80 and 160 azimuthal meshes. Then, the reference solution was obtained with Richardson extrapolation of the two results. Again, no acceleration scheme for fission source iteration was not used in the PROTEUS-NODAL and DIF3D-FDM calculations.

Table 2.4 shows that the $\mathrm{P}_{1}$ solution in $\mathrm{R}-\theta-\mathrm{Z}$ geometry agrees very well with the DIF3DFDM reference result. The use of the azimuthal node size of $17.8^{\circ}$ turned out to be sufficiently fine for this problem. As seen in the unrodded case, the $\mathrm{P}_{1}$ solution in hexagonal geometry with $9 \mathrm{~cm}$ hexagonal pitch agrees very well with the reference solution. For a comparable accuracy, the R- $\theta-\mathrm{Z}$ geometry model reduces the computational time by a factor of 7.8 relative to the hexagonal geometry model. Although the computational gain depends on the specific problem, it is obvious that an R- $\theta-\mathrm{Z}$ geometry model is more efficient and accurate than a hexagonal geometry model for cylindrical geometry problems. 


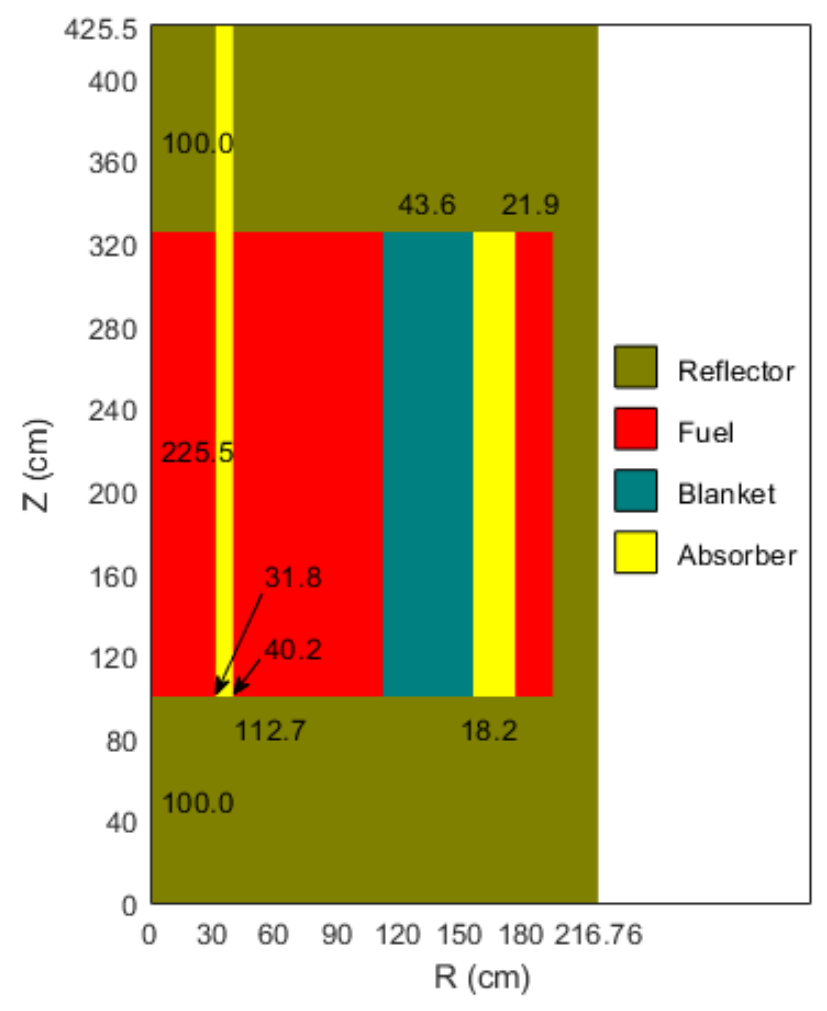

Fig. 2.4. Cross sectional view at an azimuthal angle where control rod is inserted in the simplified MSFR.
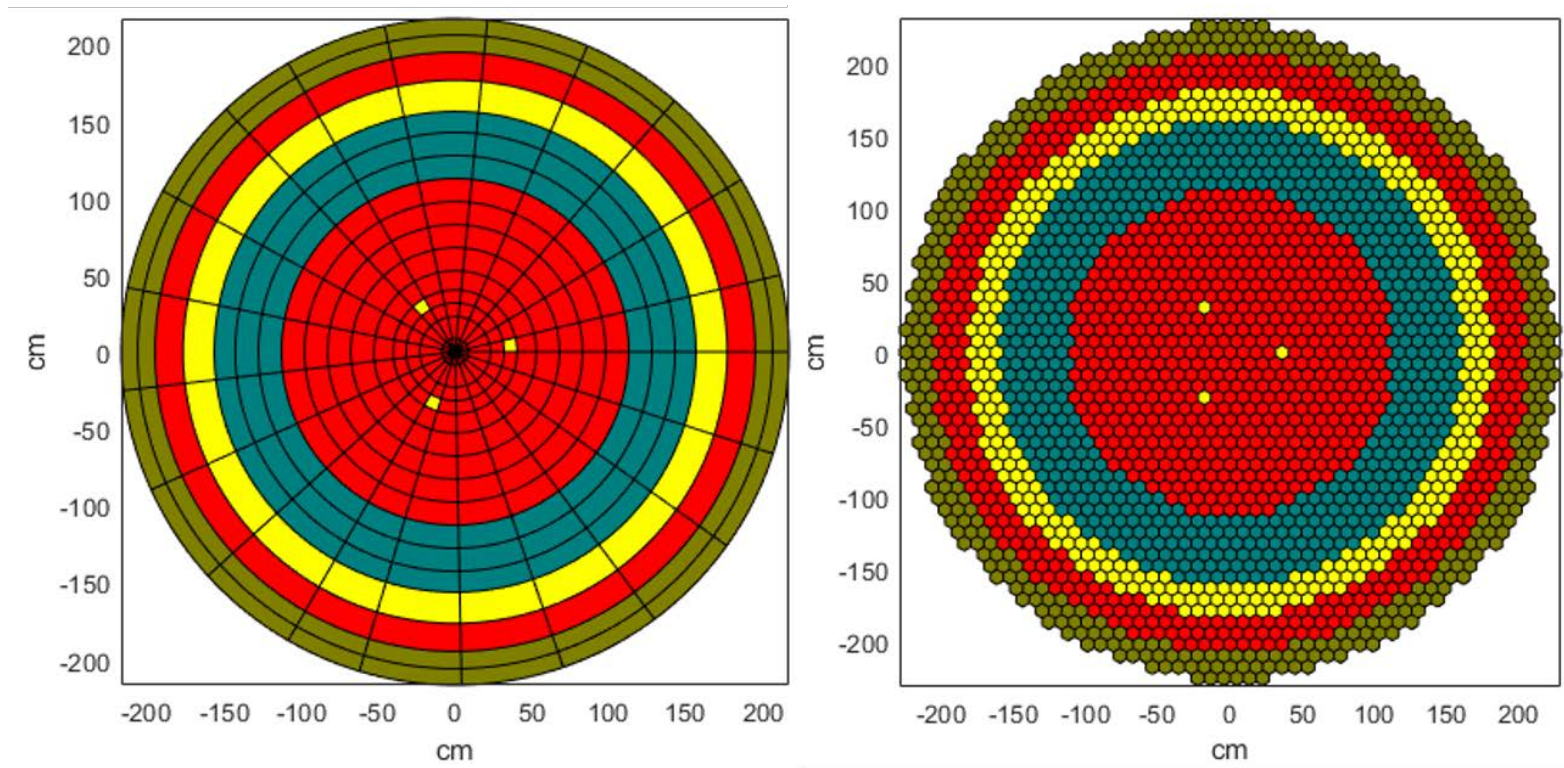

Fig. 2.5. Modified core models with control rods in $\mathrm{R}-\boldsymbol{\theta}-\mathrm{Z}$ geometry with $\Delta \theta=13.3^{\circ}$ for the control rod regions and $17.8^{\circ}$ for other regions (left) and in hexagonal geometry with 15 rings of hexagons of $9.0 \mathrm{~cm}$ pitch (right). 
Table 2.4. Eigenvalue and Power Comparison Results of Rodded Case

\begin{tabular}{|l|l|l|l|l|}
\hline & \multicolumn{1}{|c|}{ K-eff (error) } & $\begin{array}{c}\% \Delta P^{\mathrm{a}} \\
\text { RMS, MAX }\end{array}$ & $\begin{array}{c}\text { Time } \\
(\mathrm{s})\end{array}$ & $\begin{array}{l}\text { \# of Outer } \\
\text { Iterations }\end{array}$ \\
\hline FDM $\left(1.48^{\circ} \times 2 \mathrm{~cm} \times 2 \mathrm{~cm}\right)^{\mathrm{b}}$ & $0.96329(+26 \mathrm{pcm})$ & $0.15,0.80$ & 665.8 & 78 \\
\hline FDM $\left(0.74^{\circ} \times 1 \mathrm{~cm} \times 1 \mathrm{~cm}\right)$ & $0.96310(+7 \mathrm{pcm})$ & $0.039,0.20$ & 6763.9 & 101 \\
\hline Extrapolated $($ Reference $)$ & 0.96303 & - & - & \multicolumn{2}{|l|}{} \\
\hline R-Z Nodal $\left(8.9^{\circ} 10 \sim 15 \mathrm{~cm}\right)$ & $0.96304(+1 \mathrm{pcm})$ & $0.019,0.11$ & 15.3 & 41 \\
\hline R-Z Nodal $\left(17.8^{\circ}, 10 \sim 15 \mathrm{~cm}\right)$ & $0.96306(+3 \mathrm{pcm})$ & $0.039,0.17$ & 9.9 & 46 \\
\hline Hexagonal Nodal $(9.0 \mathrm{~cm})$ & $0.96306(+3 \mathrm{pcm})$ & & 77.9 & 42 \\
\hline
\end{tabular}

* All nodal calculations used $10 \mathrm{~cm}$ and $11.275 \mathrm{~cm}$ axial node sizes for the axial reflectors and core regions and the sixth and linear expansion orders for volumetric and surface basis functions, respectively.

a Averaged over a sector $\left(\Delta \theta=17.8^{\circ}\right)$ of a ring in the fuel region with $11.275 \mathrm{~cm}$ in the axial direction and $10 \sim 15 \mathrm{~cm}$ in the radial direction.

${ }^{\mathrm{b}} \Delta \theta \times \Delta r \times \Delta z$ 


\section{Development of Transient Analysis Capabilities of $\mathbf{P}_{1}$ Solver}

In the original PROTEUS-NODAL code, the transient analysis capability was limited to the $\mathrm{SP}_{3}$ solver in hexagonal geometry. In order to perform transient analyses for reactors in Cartesian (e.g., MSRE) and cylindrical (e.g., MSFR) geometries directly without geometrical approximations, the transient analysis capabilities for stationary and flowing fuels have been added to the variational nodal $\mathrm{P}_{1}$ solver that can now solve problems in Cartesian, hexagonal$Z$, triangular-Z, and R- $\theta-Z$ geometries. To improve the computational efficiency for transient analyses, a CMFD acceleration scheme was also implemented. The developed $\mathrm{P}_{1}$ transient solver was verified against the $\mathrm{SP}_{3}$ transient solver verified in the last year work using various transient problems in hexagonal geometry. The $\mathrm{P}_{1}$ transient solver in R-Z geometry was also tested by comparing the results with those of the hexagonal geometry option of the $\mathrm{P}_{1}$ solver and those of the $\mathrm{SP}_{3}$ solver.

The method to solve the TFSP for stationary fuel is first discussed. This is followed by the discussion on the modified precursor concentration equation for flowing fuel and its solution scheme. The CMFD acceleration method for transient analyses, the kinetics parameter evaluation, the thermal feedback model, and the coupling scheme of the neutronics and thermal-hydraulics calculations are described in the subsequent subsections. Then, verification test results of the implemented transient capabilities are presented. The descriptions of added input data to PROTEUS-NODAL are provided in Appendix D along with sample input files.

\subsection{Transient Fixed Source Problem}

Using the conventional notations, the time-dependent neutron diffusion equation in the multigroup form with the delayed neutron precursor concentration equations for stationary fuel can be written as

$$
\begin{gathered}
\frac{1}{v_{g}} \frac{\partial}{\partial t} \phi_{g}(\vec{r}, t)-\nabla \cdot D_{g}(\vec{r}, t) \nabla \phi_{g}(\vec{r}, t)+\Sigma_{r g}(\vec{r}, t) \phi_{g}(\vec{r}, t)=q_{g}(\vec{r}, t), \\
\frac{\partial}{\partial t} C_{k}(\vec{r}, t)+\lambda_{k} C_{k}(\vec{r}, t)=\lambda \psi_{k}(\vec{r}, t) \text { for } k=1,2, \cdots, K,
\end{gathered}
$$

where 


$$
\begin{aligned}
q_{g}(\vec{r}, t) & =\sum_{g^{\prime} \neq g}^{G} \Sigma_{s g^{\prime} g}(\vec{r}, t) \phi_{g^{\prime}}(\vec{r}, t) \\
& +\lambda \chi_{p g}(\vec{r}, t) \sum_{g^{\prime}=1}^{G} v_{p} \Sigma_{f g^{\prime}}(\vec{r}, t) \phi_{g^{\prime}}(\vec{r}, t)+\sum_{k=1}^{K} \chi_{d k g}(\vec{r}, t) \lambda_{k} C_{k}(\vec{r}, t)
\end{aligned}
$$

In Eq. (3.3), the delayed neutron precursor generation rate for delayed neutron precursor group $k$ is given by

$$
\psi_{k}(\vec{r}, t)=\sum_{g^{\prime}=1}^{G} v_{d k} \Sigma_{f g^{\prime}}(\vec{r}, t) \phi_{g^{\prime}}(\vec{r}, t)
$$

For flowing fuels, Eq. (3.3) needs to be modified to include the precursor drift as discussed in the next subsection. In this subsection, the solution scheme for the TFSP is discussed for stationary fuels. Using the implicit Euler method, Eq. (3.1) can be temporarily discretized as

$$
\frac{\phi_{g}^{n}(\vec{r})-\phi_{g}^{n-1}(\vec{r})}{v_{g} \Delta t_{n}}-\nabla \cdot D_{g}^{n}(\vec{r}) \nabla \phi_{g}^{n}(\vec{r})+\Sigma_{r g}^{n}(\vec{r}) \phi_{g}^{n}(\vec{r})=q_{g}^{n}(\vec{r})
$$

By moving the time derivative term to the right hand side, Eq. (3.5) can be written as the TFSP for given delayed neutron precursor concentrations as

$$
-\nabla \cdot D_{g}^{n}(\vec{r}) \nabla \phi_{g}^{n}(\vec{r})+\sum_{r g}^{n}(\vec{r}) \phi_{g}^{n}(\vec{r})=q_{g}^{\prime n}(\vec{r}),
$$

where

$$
\begin{aligned}
q_{g}^{\prime n}(\vec{r}) & =\frac{\phi_{g}^{n-1}(\vec{r})-\phi_{g}^{n}(\vec{r})}{v_{g} \Delta t_{n}}+\sum_{g^{\prime} \neq g}^{G} \Sigma_{s g^{\prime} g}^{n}(\vec{r}) \phi_{g^{\prime}}^{n}(\vec{r}) \\
& +\lambda \chi_{p g}(\vec{r}) \sum_{g^{\prime}=1}^{G} v_{p} \Sigma_{f g^{\prime}}^{n}(\vec{r}) \phi_{g^{\prime}}^{n}(\vec{r})+\sum_{k=1}^{K} \chi_{d k g}(\vec{r}) \lambda_{k} C_{k}^{n}(\vec{r})
\end{aligned}
$$

By approximating the precursor production rate by a quadratic function using the fission sources at previous two time nodes, Eq. (3.3) can be integrated analytically as

$$
\lambda_{k} C_{k}^{n}(\vec{r})=\lambda_{k} e^{-\lambda_{k} \Delta t_{n}} C_{k}^{n-1}(\vec{r})+\lambda\left[\Omega_{k}^{n-2} \psi_{k}^{n-2}(\vec{r})+\Omega_{k}^{n-1} \psi_{k}^{n-1}(\vec{r})+\Omega_{k}^{n} \psi_{k}^{n}(\vec{r})\right] .
$$

With $r_{n}=\Delta t_{n-1} / \Delta t_{n}$, the three parameters in Eq. (3.8) are given by 


$$
\begin{aligned}
& \Omega_{k}^{n-2}=\frac{1}{r_{n}\left(1+r_{n}\right) \lambda_{k} \Delta t_{n}}\left[\frac{2\left(1-e^{-\lambda_{k} \Delta t_{n}}\right)}{\lambda_{k} \Delta t_{n}}-\left(1+e^{-\lambda_{k} \Delta t_{n}}\right)\right], \\
& \Omega_{k}^{n-1}=\frac{1}{r_{n} \lambda_{k} \Delta t_{n}}\left[1+e^{-\lambda_{k} \Delta t_{n}}+\left(1-e^{-\lambda_{k} \Delta t_{n}}\right)\left(r_{n}-\frac{2}{\lambda_{k} \Delta t_{n}}\right)\right]-e^{-\lambda_{k} \Delta t_{n}}, \\
& \Omega_{k}^{n}=1-\frac{2}{\left(1+r_{n}\right) \lambda_{k} \Delta t_{n}}+\frac{1-e^{-\lambda_{k} \Delta t_{n}}}{\left(1+r_{n}\right) \lambda_{k} \Delta t_{n}}\left(\frac{2}{\lambda_{k} \Delta t_{n}}-r_{n}\right) .
\end{aligned}
$$

Once the group source $q_{g}^{\prime n}(\vec{r})$ is calculated, the solution for the group flux in Eq. (3.6) is obtained by solving the response matrix equations in Eq. (2.15) and Eq. (2.16) using the existing steady state solver. Since the within-group flux is included in the group source in Eq. (3.6), a few inner iterations are required between Eq. (2.15) and Eq. (2.16).

\subsection{Solution of Delayed Neutron Precursor Equation for Flowing Fuel}

For flowing fuels, the drift of delayed neutron precursors is included in the precursor balance equation as a convection term as

$$
\frac{\partial}{\partial t} C_{k}(\vec{r}, t)+\nabla \cdot\left[\vec{u}(\vec{r}, t) C_{k}(\vec{r}, t)\right]+\lambda_{k} C_{k}(\vec{r}, t)=\lambda \psi_{k}(\vec{r}, t) .
$$

For an axial velocity field $\vec{u}(\vec{r})=u(r, z) \vec{e}_{z}$, Eq. (3.10) is reduced to

$$
\frac{\partial}{\partial t} C_{k}(r, z, t)+\frac{\partial}{\partial z}\left[u(r, z, t) C_{k}(r, z, t)\right]+\lambda_{k} C_{k}(r, z, t)=\lambda \psi_{k}(r, z, t)
$$

In this study, three different ways were investigated to solve Eq. (3.11). The first approach is the analytical scheme which solves the partial differential equation (PDE) using the method of characteristics (MOC). The second approach is the semi-analytical scheme or the so-called method of lines (MoL). The basis of this method relies on converting the partial differential equation into an ordinary differential equation (ODE) and solving it using a proper ODE solver. The third approach is the finite difference method (FDM) in which the delayed neutron precursor equation is discretized in both time and space. Test results showed that only the FDM produces the null transient result correctly. The MOC and MoL solutions diverged from the steady state solution in the opposite directions although the power deviations were only fractions of percent. Thus, only the FDM is discussed in this section. The MOC and MoL are summarized in Appendix B along with some comparison of results.

In the FDM approach, the spatial derivative is approximated with the upwind finite difference scheme, while the time derivative is approximated with the forward (explicit) or backward Euler (implicit) scheme. With the backward Euler scheme for temporal 
discretization and the upwind finite difference scheme for spatial discretization, Eq. (3.11) becomes

$$
\frac{\bar{C}_{k, i j}^{n}-\bar{C}_{k, i j}^{n-1}}{\Delta t_{n}}+\frac{\bar{u}_{i j}^{n} \bar{C}_{k, i j}^{n}-\bar{u}_{i j-1}^{n} \bar{C}_{k, i j-1}^{n}}{\Delta z_{j}}+\lambda_{k} \bar{C}_{k, i j}^{n}=\lambda \psi_{k, i j}^{n} .
$$

Solving for $\bar{C}_{k, i j}^{n}$ yields

$$
\bar{C}_{k, i j}^{n}=\left(1+\lambda_{k} \Delta t_{n}+\frac{\bar{u}_{i j}^{n} \Delta t_{n}}{\Delta z_{j}}\right)^{-1}\left[\frac{\bar{u}_{i j-1}^{n} \Delta t_{n}}{\Delta z_{j}} \bar{C}_{k, i j-1}^{n}+\lambda \Delta t_{n} \psi_{k, i j}^{n}\right]
$$

The precursor concentration at the core outlet is assumed equal to the average precursor concentration of the last node in the core (upwind scheme) or it can be evaluated using the linear extrapolation of the last two nodes in the core. By assuming that precursors leaving the core are uniformly mixed and they flow back to the core except for those that decay outside the core, the boundary condition at the core inlet is evaluated as

$$
C_{k}(r, 0, t)=\frac{\int_{A_{\text {out }}} d A u(r, H, t-\tau) C_{k}(r, H, t-\tau) e^{-\lambda_{k} \tau}}{\int_{A_{\text {in }}} d A u(r, 0, t)},
$$

where $\tau$ is the time taken for precursors to pass through the core outlet and return to the core inlet.

This scheme provides an unconditionally stable solution with no restrictions on the time step size and preserves the initial delayed neutron precursor concentrations since the equation is solved in the same way for both steady state and transient analyses. Also, the main advantage of this approach is that it can be extended easily to a general velocity field in order to account for the precursor drift in the radial direction. The discretized time-dependent delayed neutron precursor equation for a general velocity field can be written as

$$
\begin{gathered}
\frac{\bar{C}_{k, i l j}^{n}-\bar{C}_{k, i l j}^{n-1}}{\Delta t_{n}}+\frac{\bar{u}_{x, i l j}^{n} \bar{C}_{k, i l j}^{n}-\bar{u}_{x, i-1, l j}^{n} \bar{C}_{k, i-1, l j}^{n}}{\Delta x_{i}}+\frac{\bar{u}_{y, i l j}^{n} \bar{C}_{k, i l j}^{n}-\bar{u}_{y, i, l-1, j}^{n} \bar{C}_{k, i, l-1, j}^{n}}{\Delta y_{l}} \\
\quad+\frac{\bar{u}_{z, i l j}^{n} \bar{C}_{k, i l j}^{n}-\bar{u}_{z, i l, j-1}^{n} \bar{C}_{k, i l, j-1}^{n}}{\Delta z_{j}}+\lambda_{k} \bar{C}_{k, i l j}^{n}=\lambda \psi_{k, i l j}^{n} .
\end{gathered}
$$

For each time step, Eq. (3.15) can be solved iteratively with the Gauss-Seidel method considering the axial and radial velocity fields. Note that if there is no radial velocity, Eq. (3.15) is reduced to Eq. (3.12). For a fast spectrum MSR, it would be important to consider the radial velocity field for transients in which the fuel velocity is decreasing as in the loss of flow accident scenario. 


\subsection{Implementation of CMFD Acceleration in Variational Nodal $P_{1}$ Solver}

The coarse mesh finite difference (CMFD) acceleration scheme was implemented in the $\mathrm{P}_{1}$ solvers to enhance the fission source convergence for the TFSP. In this section, the CMFD method for the TFSP and its performance are presented.

\subsubsection{CMFD Acceleration Method}

The CMFD acceleration method can be applied to the TFSP as in the eigenvalue problem. In this method, the neutron flux preserving interfacial net currents determined from a higher order calculation - the nodal calculation here - is sought for a given source from the previous time step. To preserve the net current, the current correction coefficients are obtained for all surfaces and groups as

$$
\hat{D}_{g, i \rightarrow u}^{n}=\frac{-\left(J_{g, i \rightarrow u}^{n}\right)^{\text {nodal }}+\tilde{D}_{g, i \rightarrow u}^{n}\left(\phi_{g}^{i, n}-\phi_{g}^{u, n}\right)^{\text {nodal }}}{\left(\phi_{g}^{i, n}+\phi_{g}^{u, n}\right)^{\text {nodal }}},
$$

where $\tilde{D}_{g, i \rightarrow u}^{n}$ is the coupling coefficient between nodes $i$ and $u$ of the conventional finite difference diffusion formulation. Then, the following CMFD equations for the TFSP are constructed by moving all the terms containing quantities at the current time step to the left hand side as

$$
\begin{aligned}
& -\sum_{u} A_{i u}\left(\tilde{D}_{g, i \rightarrow u}^{n}+\hat{D}_{g, i \rightarrow u}^{n}\right) \phi_{g}^{u, n}+\left[\sum_{u} A_{i u}\left(\tilde{D}_{g, i \rightarrow u}^{n}-\hat{D}_{g, i \rightarrow u}^{n}\right)+\left(\sum_{r, g}^{n, i}+\frac{1}{v_{g} \Delta t_{n}}\right) V_{i}\right] \phi_{g}^{i, n}, \\
& \quad-q_{g}^{i, n} V_{i}=q_{g}^{i, n-1} V_{i}
\end{aligned}
$$

where $A_{i u}$ is the area of the interface between nodes $i$ and $u, V_{i}$ is the volume of node $i$, and

$$
\begin{aligned}
& q_{g}^{i, n}=\sum_{g^{\prime} \neq g}^{G} \sum_{s g^{\prime} g}^{i, n} \phi_{g^{\prime}}^{i, n}+\lambda \chi_{p g}^{i} \sum_{g^{\prime}=1}^{G} v_{p} \Sigma_{f g^{\prime, n}}^{i, n} \phi_{g^{\prime}}^{i, n}+\sum_{k} \chi_{d k g}^{i} \Omega_{k}^{n} \psi_{k}^{i, n}, \\
& q_{g}^{i, n-1}=\sum_{k} \chi_{d k g}^{i}\left\{\lambda_{k} e^{-\lambda_{k} \Delta t_{n}} C_{k}^{i, n-1}+\lambda\left(\Omega_{k}^{n-2} \psi_{k}^{i, n-2}+\Omega_{k}^{n-1} \psi_{k}^{i, n-1}\right)\right\}+\frac{1}{v_{g} \Delta t_{n}} \phi_{g}^{i, n-1} .
\end{aligned}
$$

The entire linear system of equations for all energy groups is formed and the resulting fixed source problem is solved using the Krylov subspace method with no source iteration. The resulting fission source is used in the next outer iteration step for the nodal method to determine interfacial net currents. 


\subsubsection{Performance of CMFD Acceleration}

The performance of the CMFD acceleration was examined for steady state and transient calculations. For a steady state eigenvalue calculation, small and large MSFR core problems were solved with and without the CMFD acceleration. The results in Table 3.1 and Fig. 3.1 show that the CMFD acceleration significantly reduces the number of outer iterations for both problems. However, the total computational time is almost similar to that of the calculation without the CMFD acceleration since the mesh size of the CMFD and NODAL calculations are the same and the CMFD implementation requires a longer computational time than that of the NODAL calculations.

For transient calculations, the TWIGL benchmark problem modified by converting the original Cartesian geometry to the hexagonal one [16] was solved. At each time node, a significant reduction is achieved in both the number of outer iterations and the computational time as presented in Fig. 3.2. The number of outer iterations is reduced by a factor of 20 to 60 and the computational time is reduced by a factor of 40. The reason for this large computational gain for transient calculations is due to the fact that the entire CMFD linear system is solved only once without source iterations.

Table 3.1. Performance of CMFD for Eigenvalue Problems

\begin{tabular}{|l|l|l|l|l|}
\hline Problem & \multicolumn{2}{|l|}{ Small Core } & Large Core \\
\hline No. Hexagonal Assemblies & 19 & 625 \\
\hline No. Energy Groups & 9 & 33 & \multicolumn{2}{l|}{} \\
\hline & w/o CMFD & w/ CMFD & w/o CMFD & w/ CMFD \\
\hline Eigenvalue & 1.000903 & 1.000903 & 1.022056 & 1.022056 \\
\hline Total Time (s) & 0.972 & 0.815 & 287.15 & 304.55 \\
\hline Number of Outer Iterations & 56 & 11 & 53 & 17 \\
\hline
\end{tabular}




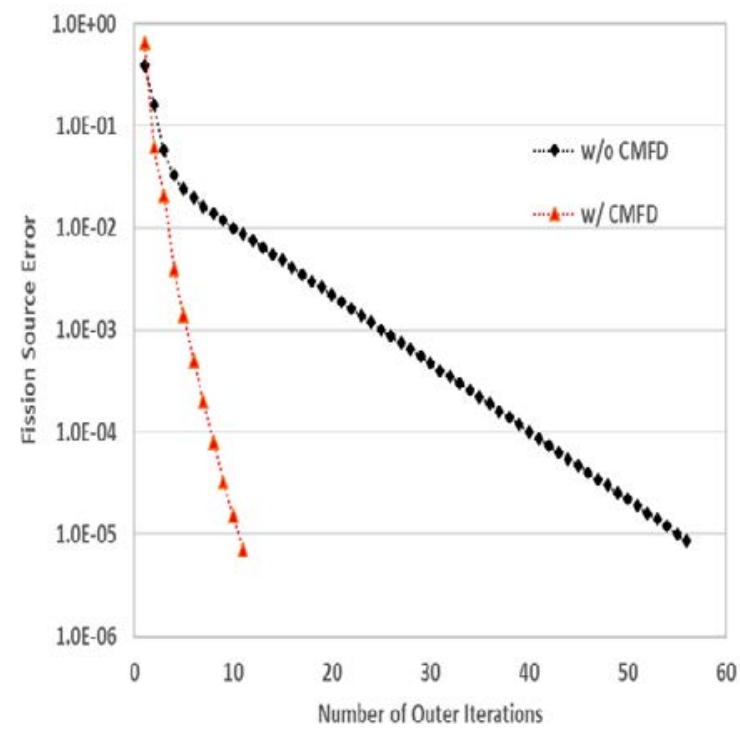

(a)

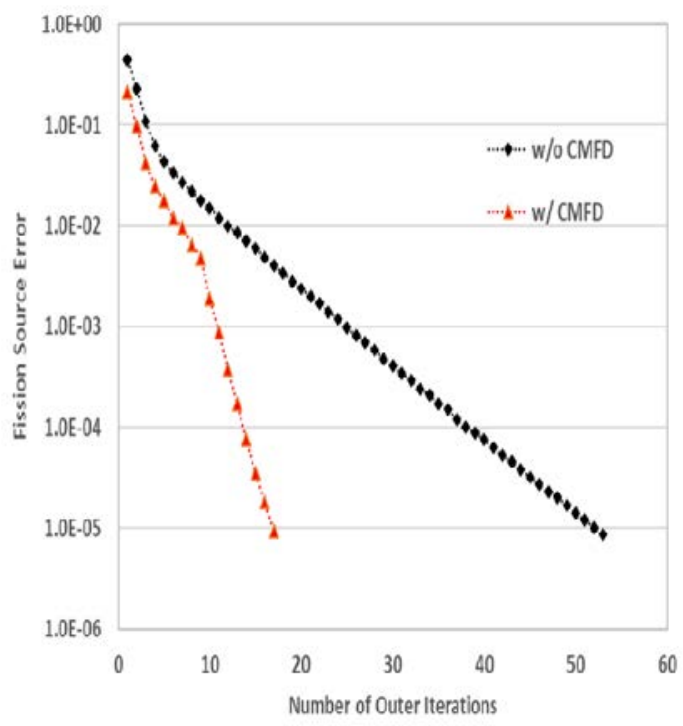

(b)

Fig. 3.1. Comparison of error reduction in fission source with and without CMFD for eigenvalue problems of (a) small and (b) large MSFR cores.

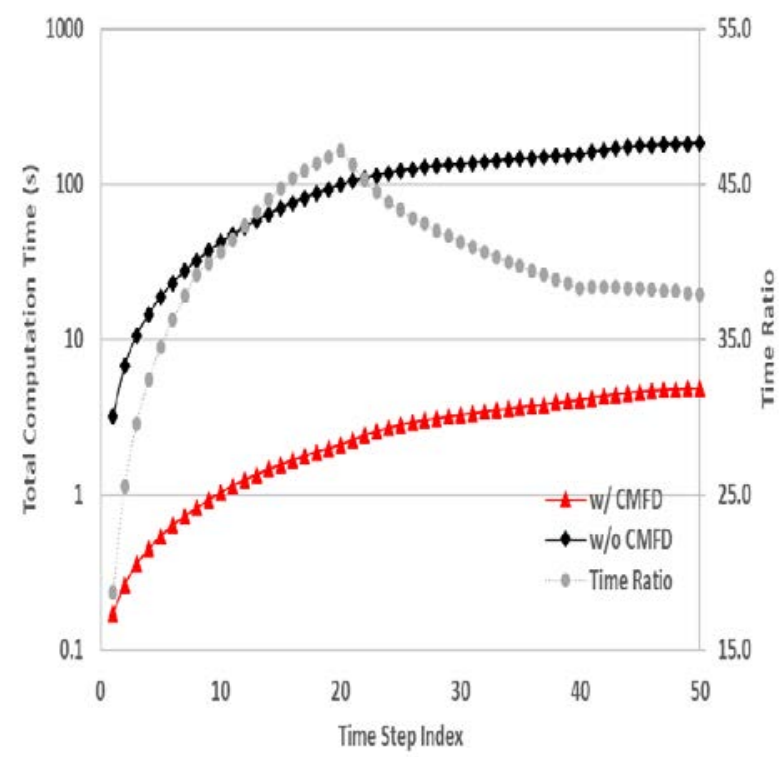

(a)

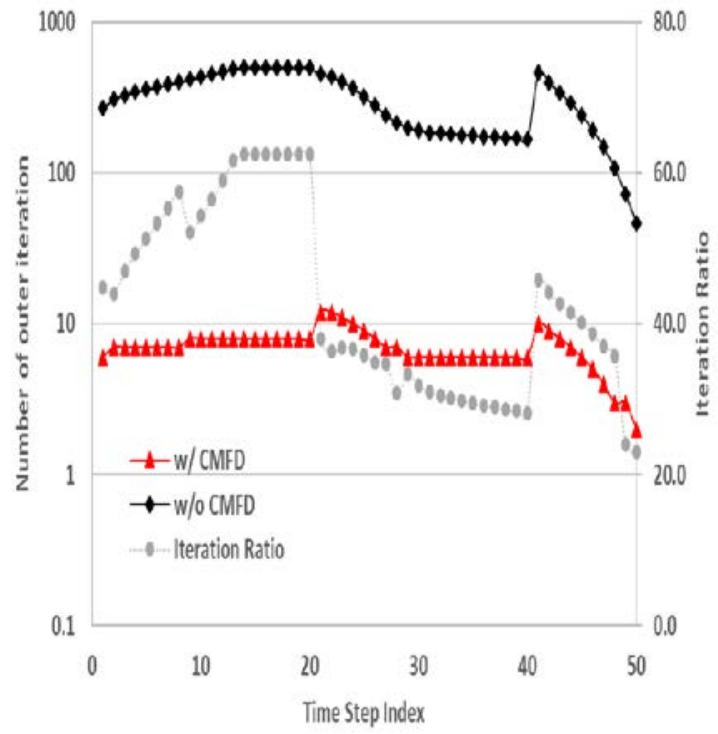

(b)

Fig. 3.2. Comparison of (a) the computational time and (b) the number of outer iterations at each time step with and without CMFD for the modified TWIGL benchmark problem. 


\subsection{Evaluation of Kinetics Parameters}

In order to compare with the reported kinetic parameters of benchmark problems, the capabilities to calculate the steady state adjoint flux and the effective delayed neutron fraction and the prompt neutron generation time have been added to the PROTEUS-NODAL code.

\subsubsection{Adjoint Flux and Kinetics Parameters}

At the steady state, the neutron diffusion equation in Eq. (3.1) and the precursor concentration equation for a flowing fuel in Eq. (3.10) are reduced to

$$
\begin{gathered}
-\nabla \cdot D_{g}(\vec{r}, t) \nabla \phi_{g}(\vec{r})+\sum_{t g}(\vec{r}) \phi_{g}(\vec{r})-\sum_{g^{\prime}=1}^{G} \sum_{s g^{\prime} \rightarrow g}(\vec{r}) \phi_{g^{\prime}}(\vec{r}) \\
=\lambda \chi_{p g}(\vec{r}) \sum_{g^{\prime}=1}^{G} v_{p} \Sigma_{f g^{\prime}}(\vec{r}) \phi_{g^{\prime}}(\vec{r})+\sum_{k=1}^{K} \chi_{d k g}(\vec{r}) \lambda_{k} C_{k}(\vec{r}) \\
\nabla \cdot\left[\vec{u}(\vec{r}) C_{k}(\vec{r})\right]+\lambda_{k} C_{k}(\vec{r})=\lambda \psi_{k}(\vec{r}), \quad k=1,2, \cdots, K,
\end{gathered}
$$

where

$$
\psi_{k}(\vec{r})=\sum_{g^{\prime}=1}^{G} v_{d k} \Sigma_{f g^{\prime}}(\vec{r}) \phi_{g^{\prime}}(\vec{r}) .
$$

The adjoint system of equations to the coupled system of equations in Eq. (3.19) and Eq. (3.20) can be obtained as

$$
\begin{gathered}
-\nabla \cdot D_{g}(\vec{r}) \nabla \phi_{g}^{*}(\vec{r})+\Sigma_{t g}(\vec{r}) \phi_{g}^{*}(\vec{r})-\sum_{g^{\prime}=1}^{G} \Sigma_{s g \rightarrow g^{\prime}}(\vec{r}) \phi_{g^{\prime}}^{*}(\vec{r}) \\
=\lambda\left[v_{p} \Sigma_{f g}(\vec{r}) \sum_{g^{\prime}=1}^{G} \chi_{p g^{\prime}}(\vec{r}) \phi_{g^{\prime}}^{*}(\vec{r})+\sum_{k=1}^{K} v_{d k} \Sigma_{f g}(\vec{r}) C_{k}^{*}(\vec{r})\right], \quad g=1,2, \cdots, G \\
-\nabla \cdot\left[u(\vec{r}) C_{k}^{*}(\vec{r})\right]+\lambda_{k} C_{k}^{*}(\vec{r})=\lambda \psi_{k}^{*}(\vec{r}), \quad k=1,2, \cdots, K,
\end{gathered}
$$

where

$$
\psi_{k}^{*}(\vec{r})=\sum_{g^{\prime}=1}^{G} \chi_{d k g^{\prime}}(\vec{r}) \phi_{g^{\prime}}^{*}(\vec{r}) .
$$

Because of the negative sign in the precursor convection term in Eq. (3.23), this equation needs to be solved in the opposite direction with a boundary condition at the core outlet that can be represented as 


$$
C_{k}^{*}(r, H)=\frac{\int_{A_{n}} d A u(r, 0) C_{k}^{*}(r, 0) e^{-\lambda_{k} \tau}}{\int_{A_{\text {out }}} d A u(r, H)} .
$$

where the position vector $\vec{r}$ is separated into the radial and axial variables. For a $1 \mathrm{D}$ axial flow, the adjoint precursor concentration equation can be solved analytically in terms of the adjoint precursor concentration at the outlet as

$$
u(r, z) C_{k}^{*}(r, z)=u(r, H) C_{k}^{*}(r, H) e^{-\lambda_{k} \int_{z}^{H} \frac{d z^{\prime}}{u\left(r, z^{\prime}\right)}}+\lambda \int_{z}^{H} d z^{\prime} \psi_{k}^{*}\left(r, z^{\prime}\right) e^{-\lambda_{k} \int_{z}^{z^{\prime}} \frac{d z^{\prime \prime}}{u\left(r, z^{\prime \prime}\right)}} .
$$

For a multi-dimensional flow, Eq. (3.23) can be solved using the FDM discussed in Section 3.2.

Using the adjoint flux, the importance-weighted quasi-stationary source of neutrons from fission and precursor decay can be obtained as

$$
F(t)=\sum_{i} V_{i} \sum_{g=1}^{G} \phi_{g, i}^{*} \chi_{p g, i} \sum_{g^{\prime}=1}^{G} v_{p} \Sigma_{f g^{\prime}, i}(t) \phi_{g^{\prime}, i}(t)+\sum_{i} V_{i} \sum_{g=1}^{G} \phi_{g, i}^{*} \sum_{k=1}^{K} \chi_{d k g, i} \lambda_{k} C_{k, i}(t)
$$

where $V_{i}$ is the volume of node $i$ and the other notations are conventional. On the right hand side of Eq. (3.27), the first term represents the total importance of prompt neutrons produced by fission and the second term represents the total importance of delayed neutrons produced by precursor decay. Therefore, the effective delayed neutron fraction for a circulating fuel can be calculated as $[17,18]$

$$
\beta_{e f f}^{\text {circ }}(t)=\frac{\sum_{i} V_{i} \sum_{g=1}^{G} \phi_{g, i}^{*} \sum_{k=1}^{K} \chi_{d k g} \lambda_{k} C_{k, i}(t)}{\sum_{i} V_{i} \sum_{g=1}^{G} \phi_{g, i}^{*} \chi_{p g, i} \sum_{g^{\prime}=1}^{G} v_{p} \Sigma_{f g^{\prime}, i}(t) \phi_{g^{\prime}, i}(t)+\sum_{i} V_{i} \sum_{g=1}^{G} \phi_{g, i}^{*} \sum_{k=1}^{K} \chi_{d k g, i} \lambda_{k} C_{k, i}(t)},
$$

Similarly, the prompt neutron generation time for a circulating fuel can be determined as

$$
\Lambda^{\text {circ }}(t)=\frac{\sum_{i} V_{i} \sum_{g=1}^{G} \phi_{g, i}^{*} v_{g}^{-1} \phi_{g, i}(t)}{\sum_{i} V_{i} \sum_{g=1}^{G} \phi_{g, i}^{*} \chi_{p g, i} \sum_{g^{\prime}=1}^{G} v_{p} \Sigma_{f g^{\prime}, i}(t) \phi_{g^{\prime}, i}(t)+\sum_{i} V_{i} \sum_{g=1}^{G} \phi_{g, i}^{*} \sum_{k=1}^{K} \chi_{d k g, i} \lambda_{k} C_{k, i}(t)} .
$$

It is noted that in an MSR, the delayed neutron production by precursor decay and the precursor production from fission should be differentiated since delayed neutron precursors move to other positions before they decay. 
For a stationary fuel, delayed neutron precursors decay at the same position where they are produced, and thus the number of delayed neutrons produced at a position is equal to the number of precursors produced by fission. Therefore, the total number of neutrons can be determined from the quasi-stationary total fission neutrons. As a result, the effective delayed neutron fraction and the prompt neutron generation time can be determined as

$$
\begin{gathered}
\beta_{e f f}(t)=\frac{\sum_{i} V_{i} \sum_{g=1}^{G} \phi_{g, i}^{*} \sum_{k=1}^{K} \chi_{d k g} \sum_{g^{\prime}=1}^{G} v_{d k} \Sigma_{f g^{\prime}} \phi_{g^{\prime}, i}}{\sum_{i} V_{i} \sum_{g=1}^{G} \phi_{g, i}^{*} \chi_{g} \sum_{g^{\prime}=1}^{G} v \Sigma_{f g^{\prime}} \phi_{g^{\prime}, i}}, \\
\Lambda(t)=\frac{\sum_{i} V_{i} \sum_{g=1}^{G} \phi_{g, i}^{*} V_{g}^{-1} \phi_{g, i}}{\sum_{i} V_{i} \sum_{g=1}^{G} \phi_{g, i}^{*} \chi_{g} \sum_{g^{\prime}=1}^{G} v \sum_{f g^{\prime}} \phi_{g^{\prime}, i}} .
\end{gathered}
$$

The reactivity is the importance-weighted neutron balance, i.e., the importance-weighted net neutron production due to neutron interactions with nuclides, leakage, and precursor decays, relative to the importance-weighted total neutron source. The reactivity for a circulating fuel can be divided into two components: one is the prompt fission neutron production minus the loss due to absorption and leakage, and the other is the delayed neutron production as

$$
\begin{aligned}
\rho^{\text {circ }}(t) & =\frac{\sum_{i} \sum_{g=1}^{G} \phi_{g, i}^{*}\left[\left(\chi_{p g, i} \sum_{g^{\prime}=1}^{G} v_{p} \Sigma_{f g^{\prime}, i} \phi_{g^{\prime}, i}-\sum_{t g, i} \phi_{g, i}+\sum_{g^{\prime}=1}^{G} \sum_{s g^{\prime} \rightarrow g, i} \phi_{g^{\prime}, i}\right) V_{i}-\sum_{\gamma} J_{g, \gamma i} A_{\gamma i}\right]}{\sum_{i} V_{i} \sum_{g=1}^{G} \phi_{g, i}^{*} \chi_{p g, i} \sum_{g^{\prime}=1}^{G} v_{p} \Sigma_{f g^{\prime}, i}(t) \phi_{g^{\prime}, i}(t)+\sum_{i} V_{i} \sum_{g=1}^{G} \phi_{g, i}^{*} \sum_{k=1}^{K} \chi_{d k g, i} \lambda_{k} C_{k, i}(t)}, \\
& +\beta_{\text {eff }}^{\text {circ }}(t)
\end{aligned}
$$

where $J_{g, \gamma i}$ is the net current of the surface $\gamma$ of node $i$ and $A_{\gamma i}$ is the surface area. It can be seen that the change in the effective delayed neutron fraction due to the precursor decay outside of the core is directly reflected in the reactivity.

\subsubsection{Verification Tests of Kinetics Parameters}

The adjoint flux solution capability implemented in PROTEUS-NODAL was verified by calculating the safety parameters of the MSFR benchmark problem and by comparing the resulting values with the other code results reported in the EVOL report [19]. The MSFR benchmark problem is described in Appendix A. The effective delayed neutron fractions of 
stationary $\left(\beta_{\mathrm{eff}}\right)$ and flowing fuel salts $\left(\beta_{\mathrm{circ}}\right)$, and the prompt neutron generation time were calculated for the ${ }^{233} \mathrm{U}$-started and TRU-started MSFR.

Table 3.2 compares the kinetics parameters calculated with PROTEUS-NODAL with the values reported in the EVOL report. The effective delayed neutron fraction of the flowing fuel salt is almost half of that of the stationary fuel salt. This is because the residence time of the fuel salt in the core equals to the transit time of the fuel salt in the external loop. Both the effective delayed neutron fraction and the prompt neutron generation time calculated with PROTEUS-NODAL are consistent with other reported values.

Table 3.2. Calculated Safety Parameters for MSFR Benchmark Problem

\begin{tabular}{|c|c|c|c|c|c|c|}
\hline \multicolumn{7}{|c|}{ U-233 Started } \\
\hline Institute & LPSC & POLITO & POLIMI & TUDelft & Purdue & PROTEUS \\
\hline XS library & ENDF/B-VI & JEFF-3.1.1 & JEFF-3.1 & ENDF/B-VII & ENDF/B-VII.1 & ENDF/B-VII.0 \\
\hline$\beta_{\text {eff }}(p c m)$ & 320.0 & 305.0 & 305.0 & 290.0 & 318.8 & 317.7 \\
\hline$\beta_{\text {circ }}(\mathrm{pcm})$ & 169.5 & 117.3 & 146.0 & 124.6 & 142.1 & 141.2 \\
\hline$\beta_{\text {circ }} / \beta_{\text {eff }}$ & 0.529 & 0.385 & 0.479 & 0.430 & 0.446 & 0.444 \\
\hline$\beta_{\text {loss }}(\mathrm{pcm})$ & 150.5 & 187.7 & 159.0 & 165.4 & 176.7 & 176.6 \\
\hline$\beta_{\text {loss }} / \beta_{\text {eff }}$ & 0.470 & 0.615 & 0.521 & 0.570 & 0.554 & 0.556 \\
\hline$\Lambda(\mu s)$ & 1.200 & 0.971 & 1.090 & 1.150 & - & 1.103 \\
\hline \multicolumn{7}{|c|}{ TRU-Started } \\
\hline Institute & LPSC & POLITO & POLIMI & TUDelft & Purdue & PROTEUS \\
\hline XS library & ENDF/B-VI & JEFF-3.1.1 & JEFF-3.1 & ENDF/B-VII & ENDF/B-VII.1 & ENDF/B-VII.0 \\
\hline$\beta_{\text {eff }}(p c m)$ & 312.8 & 301.0 & 302.0 & - & 298.8 & 294.6 \\
\hline$\beta_{\text {circ }}(\mathrm{pcm})$ & 165.5 & - & 147.0 & - & 138.5 & 135.0 \\
\hline$\beta_{\text {circ }} / \beta_{\text {eff }}$ & 0.529 & - & 0.487 & - & 0.464 & 1.464 \\
\hline$\beta_{\text {loss }}(\mathrm{pcm})$ & 147.3 & - & 155.0 & - & 160.3 & 159.6 \\
\hline$\beta_{\text {loss }} / \beta_{\text {eff }}$ & 0.471 & - & 0.513 & - & 0.536 & 0.542 \\
\hline$\Lambda(\mu \mathrm{s})$ & 0.900 & 0.783 & 0.650 & - & - & 0.623 \\
\hline
\end{tabular}

Additionally, the forward and adjoint delayed neutron precursor distributions are compared for stationary and flowing fuels. Fig. 3.3 compares the axial distributions of 
precursor concentrations for all the six precursor groups, and Fig. 3.4 compares the axial distributions of adjoint precursor concentrations. Fig. 3.3 shows that the precursor concentration of the stationary fuel is symmetric about the core mid-plane for all the six precursor groups. On the other hand, for the flowing fuel, the first two precursor groups show almost a uniform distribution of precursor concentrations due to their relatively large halflives, but the precursor concentration of group five varies significantly from the core inlet to the core outlet.
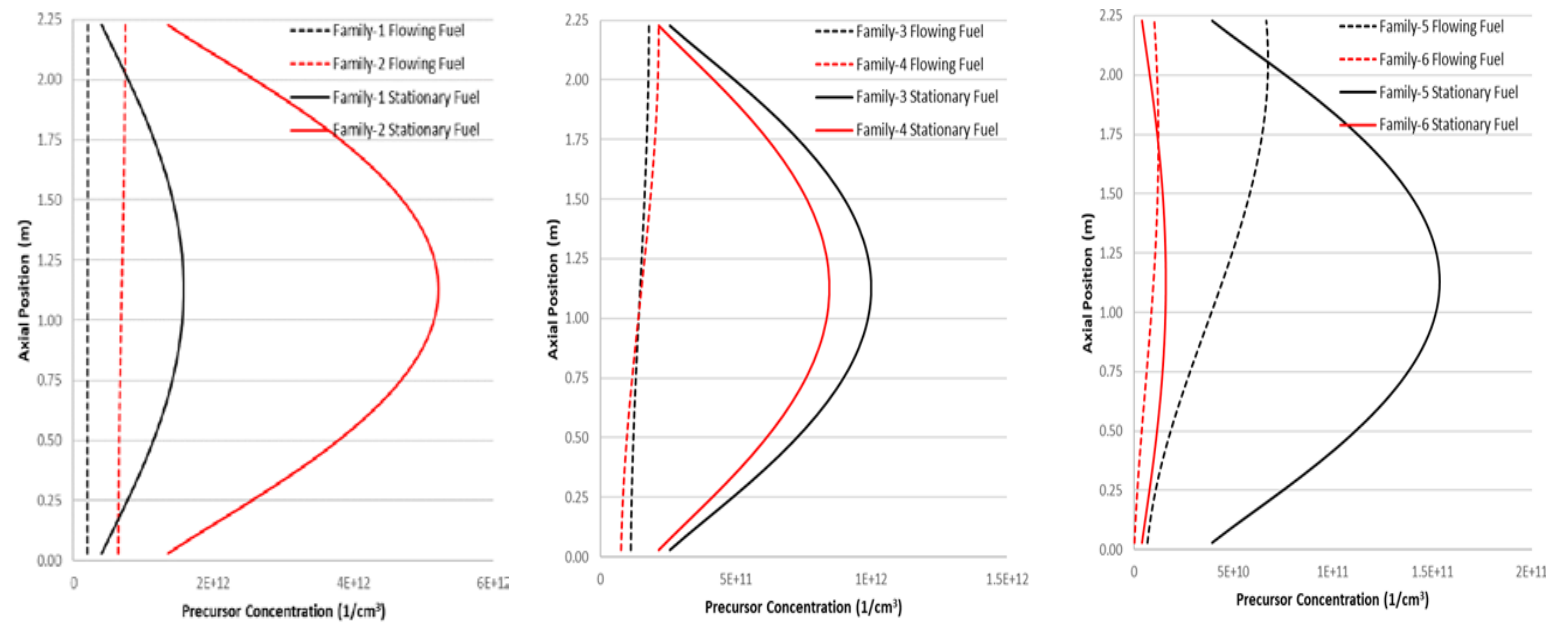

Fig. 3.3. Delayed neutron precursor distribution in stationary and flowing fuels.
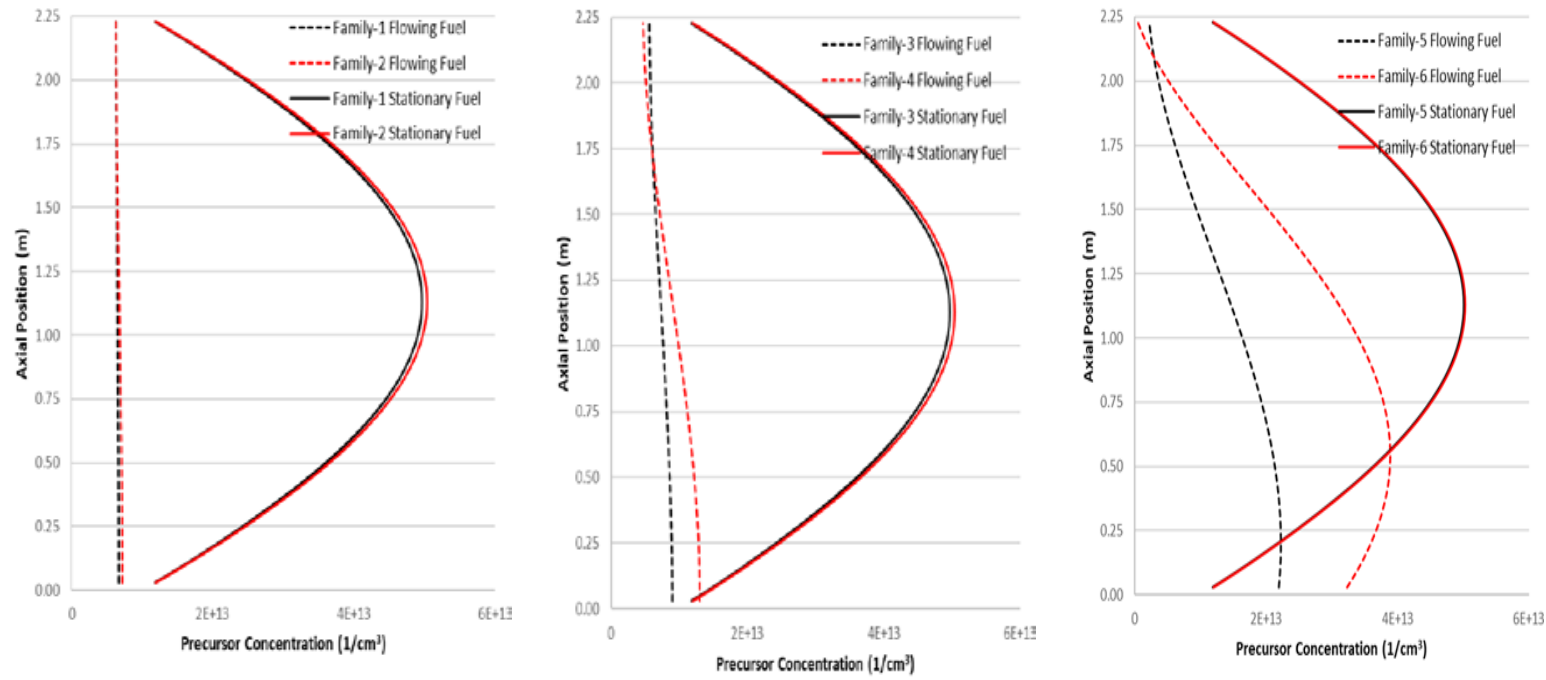

Fig. 3.4. Delayed neutron precursor importance in stationary and flowing fuels.

Fig. 3.4 also shows that the adjoint precursor concentration of the stationary fuel is symmetric about the core mid-plane for all the six precursor groups. It is noted that for the stationary fuel, the axial importance distributions of the six precursor groups are similar to each other since the delayed neutron spectra are similar. On the other hand, in the flowing 
fuel, the axial importance distributions of the six precursor groups are very different because of the different decay constants. The adjoint precursor concentrations of groups 3 to 6 show significant axial variations. For the groups 1 and 2, the adjoint precursor distributions are almost constant throughout the active core.

\subsection{Thermal Feedback Calculation}

This section explains the coupled neutronics and thermal-hydraulics calculation scheme to account for thermal feedback. The cross section functionalization scheme for thermal feedback is discussed first, and then the overall computational procedure of the coupled calculation for thermal feedback is discussed.

\subsubsection{Cross Section Functionalization}

The calculation scheme for thermal feedback has been developed for fast and thermal spectrum reactor applications. To functionalize cross sections, multigroup cross sections were prepared using the multigroup cross section generation code $\mathrm{MC}^{2}-3$ [20] or Monte Carlo codes Serpent [21] and OpenMC [22]. The isotopic cross section dataset ISOTXS and the delayed neutron cross section dataset DLAYXS are generated using $\mathrm{MC}^{2}-3$ directly or using the utility code GenISOTXS [23] that converts the output data of Serpent or OpenMC to ISOTXS. In this study, a capability to convert the Serpent or OpenMC output to DLAYXS was added to GenISOTXS. Cross sections for the fast and thermal spectrum MSRs were functionalized differently based on the temperature dependence of cross sections for the Doppler effects and the existence of a moderator.

In a fast spectrum MSR, the reactivity feedback is due to the Doppler effects and the change of fuel density. In large fast reactors, the Doppler effects is approximately proportional to $1 / T$ [24] and thus the microscopic cross section can be represented as a linear function of $\ln (T)$.

$$
\frac{d \sigma}{d T}=\frac{\alpha}{T} \rightarrow \sigma(T)=\sigma\left(T_{0}\right)+\alpha \ln \left(T / T_{0}\right)
$$

Microscopic cross sections are prepared at two temperatures $T_{L}$ and $T_{H}$. After each thermal-hydraulics calculation, the cross sections are updated as

$$
\Sigma(T)=\frac{\rho(T)}{\rho\left(T_{0}\right)} \sum_{i} N_{i}\left(T_{0}\right)\left[\varepsilon_{L} \sigma_{i}\left(T_{L}\right)+\varepsilon_{H} \sigma_{i}\left(T_{H}\right)\right],
$$

where

$$
\varepsilon_{L}=\frac{\ln \left(T_{H} / T\right)}{\ln \left(T_{H} / T_{L}\right)}, \quad \varepsilon_{H}=1-\varepsilon_{L} .
$$


In a thermal spectrum MSR, cross sections depend on the moderator temperature as well as the fuel temperature. The resonance integral in thermal reactors is approximately proportional to $\sqrt{T}$ and thus the microscopic cross sections of fuel isotopes are represented

as a linear function of $\sqrt{T}$. On the other hand, the moderator temperature dependency is represented as a linear function of $T$. After each thermal-hydraulics calculation, the cross sections are updated as

$$
\begin{array}{r}
\Sigma\left(T_{F}, T_{M}\right)=\frac{\rho_{F}\left(T_{F}\right)}{\rho_{F}\left(T_{F 0}\right)} \sum_{i} N_{i}^{F}\left(T_{F 0}\right)\left[\varepsilon_{L}^{F} \sigma_{i}^{F}\left(T_{L}^{F}\right)+\varepsilon_{H}^{F} \sigma_{i}^{F}\left(T_{H}^{F}\right)\right] \\
+\sum_{j} N_{j}^{M}\left[\varepsilon_{L}^{M} \sigma_{j}^{M}\left(T_{L}^{M}\right)+\varepsilon_{H}^{M} \sigma_{j}^{M}\left(T_{L}^{H}\right)\right],
\end{array}
$$

where

$$
\begin{array}{ll}
\varepsilon_{L}^{F}=\left(\sqrt{T_{F}^{H}}-\sqrt{T_{F}}\right) /\left(\sqrt{T_{F}^{H}}-\sqrt{T_{F}^{L}}\right), & \varepsilon_{L}^{M}=\left(T_{M}^{H}-T_{M}\right) /\left(T_{M}^{H}-T_{M}^{L}\right), \\
\varepsilon_{H}^{F}=1-\varepsilon_{L}^{F}, & \varepsilon_{H}^{M}=1-\varepsilon_{L}^{M} .
\end{array}
$$

\subsubsection{Calculation Flow}

The coupling schemes of the neutronics and the thermal-hydraulics calculations for steady state and transient analyses are illustrated in Fig. 3.5. In steady state calculations, the neutronics and thermal-hydraulics equations are tightly coupled and solved iteratively until the power and temperature solutions converge. The initial power distribution in the core is determined by performing the nodal calculation using PROTEUS-NODAL with uniform temperature and velocity fields. At the end of each power iteration of the nodal calculation, a thermal-hydraulics calculation is performed to determine the fuel salt temperature, density, and velocity fields in the core region using the updated power distribution. Then, the cross sections in the core region are updated using the calculated fuel salt temperature distribution. Using the updated cross sections and the salt velocity field, the nodal calculation is performed again. This process continues until the power and temperature distributions converge.

In transient calculations, the neutronics and thermal-hydraulics calculations are performed once for each time step. At the beginning of each time step, the temperature, velocity and density distributions at the previous time step are used to determine the cross sections. Using the cross sections, the neutronics calculation is performed to update the power distribution. Using the updated power distribution, the thermal-hydraulics calculation is performed to calculate the new fuel salt temperature, density, and velocity fields. Then, these updated 
temperature, density, and velocity distributions are used in the feedback calculation in the next time step until the end of the time step is reached.

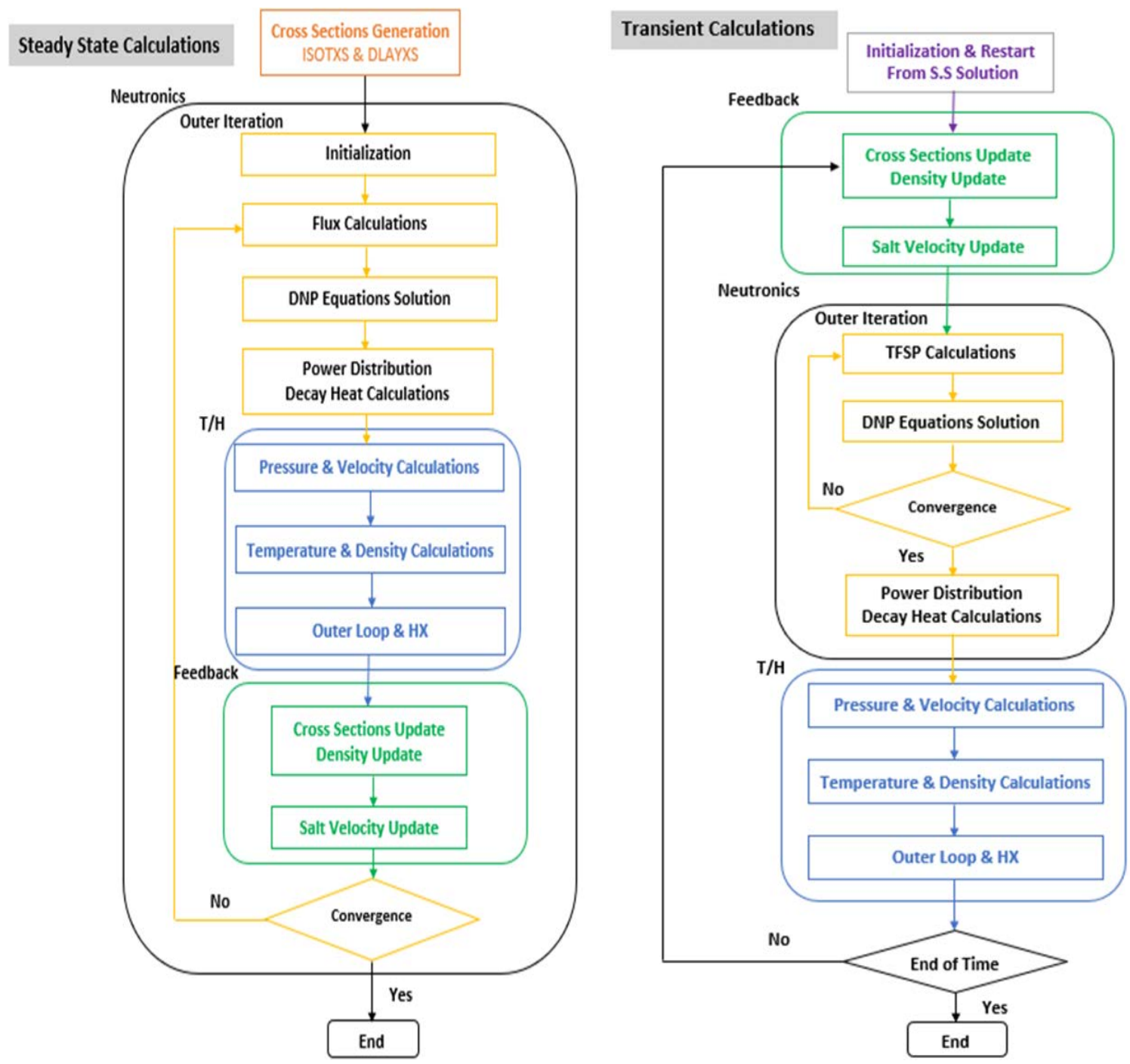

Fig. 3.5. Overall coupling schemes of neutronics and thermal-hydraulics calculations.

\subsection{Verification Tests of $P_{1}$ Transient Solver}

In this section, verification test results of the $\mathrm{P}_{1}$ transient solver are discussed. As the $\mathrm{SP}_{3}$ transient solver was verified in the last year work [9], the results of the P1 transient solver were compared to those of the $\mathrm{SP}_{3}$ transient solver. For consistent comparison, using the $\mathrm{P}_{1}$ and $\mathrm{SP}_{3}$ solvers, transient calculations were first performed in hexagonal geometry without invoking the thermal feedback module. These tests were performed for both stationary and flowing fuels. Then, the same transients were analyzed in R-Z geometry with thermal feedback and the results were compared with those of the $\mathrm{P}_{1}$ and $\mathrm{SP}_{3}$ transient solvers for 
hexagonal geometry. The thermal feedback calculation was performed using the standalone thermal-hydraulics solver of PROTEUS-NODAL, which is discussed in Appendix C.

\subsubsection{Verification Tests without Thermal Feedback}

The $\mathrm{P}_{1}$ transient solver was first verified with the modified TWIGL benchmark problem [16]. The same hexagonal geometry core of six rings of $22.5 \mathrm{~cm}$-pitch hexagons was used for both the $\mathrm{P}_{1}$ and $\mathrm{SP}_{3}$ solver. The Doppler feedback effects were taken into account using the provided sets of cross sections without invoking the standalone thermal-hydraulics calculation module. Fig. 3.6 compares the calculation results. The $\mathrm{P}_{1}$ solution agrees very well with the $\mathrm{SP}_{3}$ solution within $1 \%$ error. As the same hexagonal geometry model was used, this difference is solely due to the transport effect. As the diffusion approximation overestimates leakage in general, the $\mathrm{P}_{1}$ transient solver yields a $1 \%$ smaller peak power than the $\mathrm{SP}_{3}$ solver.

Next tests were performed with a MSFR problem of reduced size in order to reduce the computational time. Step reactivity insertion transients were simulated for both stationary and flowing fuels. For the stationary fuel, two step-reactivity insertions of $+100 \mathrm{pcm}$ and 100 pcm were investigated by adjusting the fuel concentration. Fig. 3.7 and Fig. 3.8 show the power changes in the first one second for the positive and negative reactivity insertion problems, respectively. For both the positive and negative reactivity insertion problems, the $\mathrm{P}_{1}$ transient solution agrees well with the $\mathrm{SP}_{3}$ transient result. An about $1 \%$ difference is observed during the prompt jump period and the error remains within 1.5\%.

For a flowing fuel, the reactivity insertion was simulated by adjusting the fuel velocity in the core. An increased fuel velocity increases the loss of delayed neutrons and thus decreases the reactivity. On the contrary, a decreased fuel velocity increases the reactivity due to a decreased loss of delayed neutrons. Fig. 3.9 shows the power response to the step increase in the fuel velocity. The power decreases initially and then shows an oscillatory behavior of 4.0 $\mathrm{s}$ period because the fuel leaving the core flows back to the core after $2.0 \mathrm{~s}$. The $\mathrm{P}_{1}$ solution agrees well with the $\mathrm{SP}_{3}$ solution within $1.0 \%$. The power response to the step velocity decrease is shown in Fig. 3.10. The decreased fuel velocity introduces a positive reactivity and thus the power increases exponentially. The $\mathrm{P}_{1}$ solution agrees well with the $\mathrm{SP}_{3}$ solution within $0.6 \%$. These results demonstrate that the $\mathrm{P}_{1}$ transient solver is working properly. 


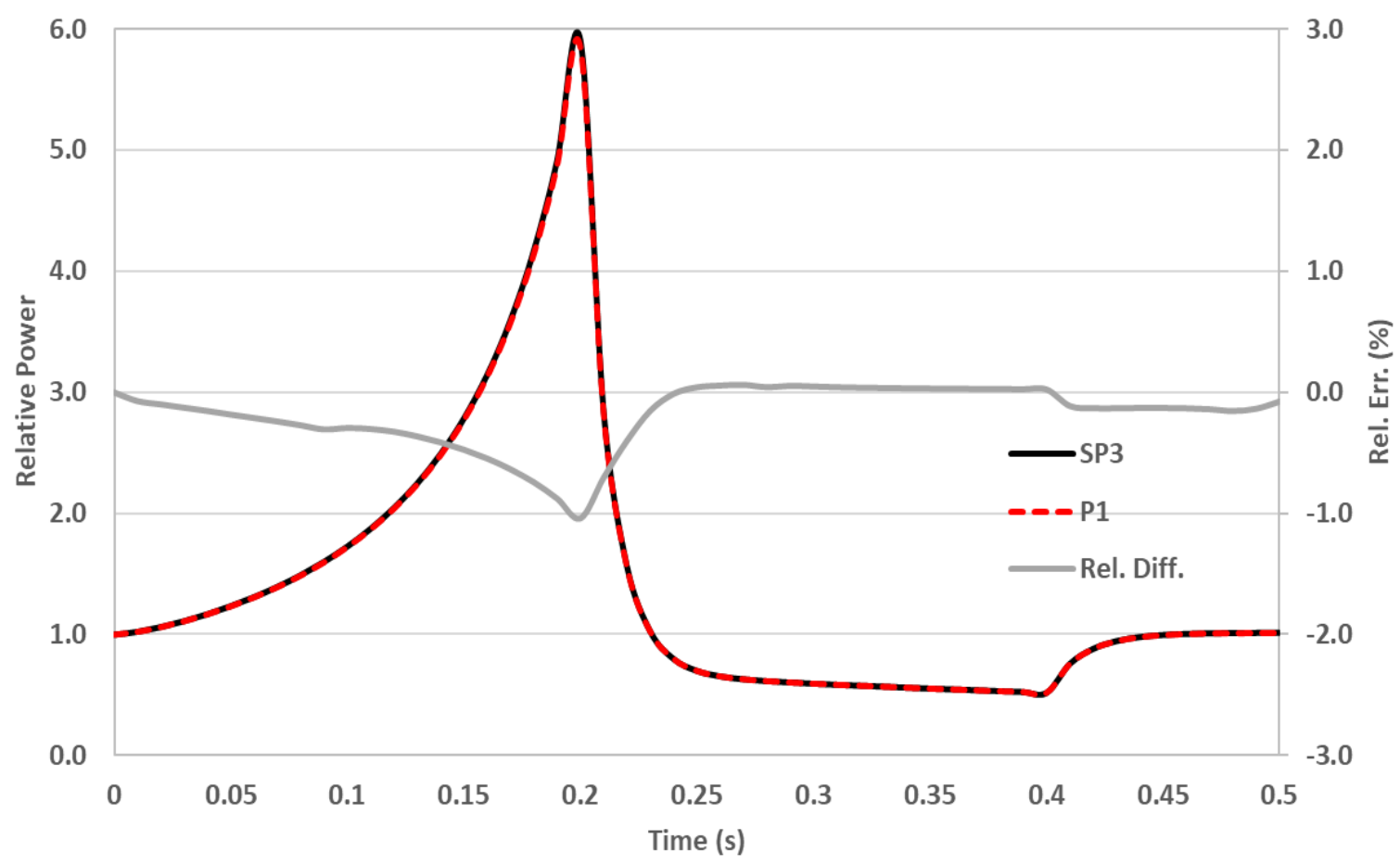

Fig. 3.6. Power change with time for the modified TWIGL benchmark problem.

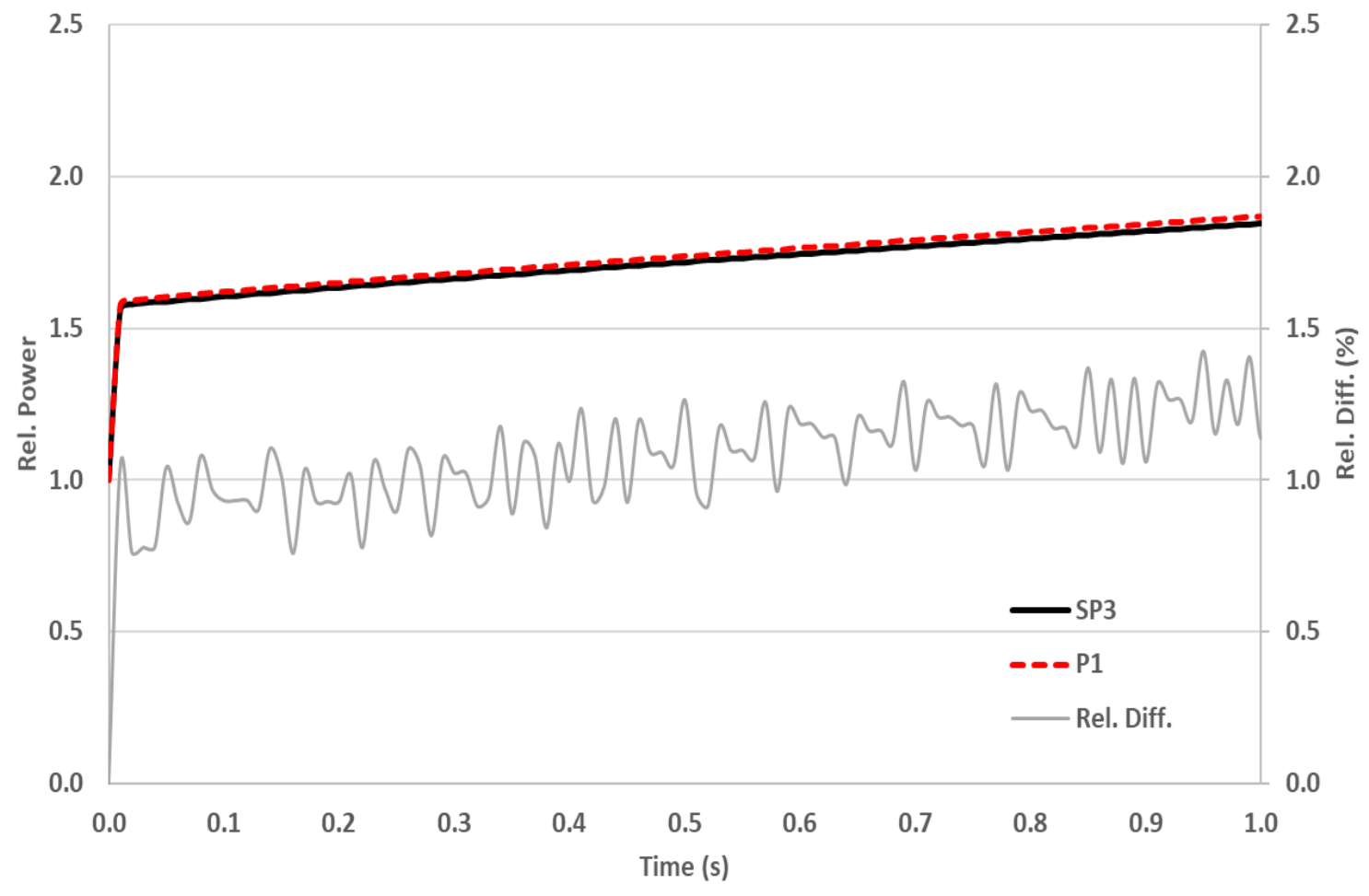

Fig. 3.7. Power comparison after the positive step reactivity insertion for the stationary fuel. 


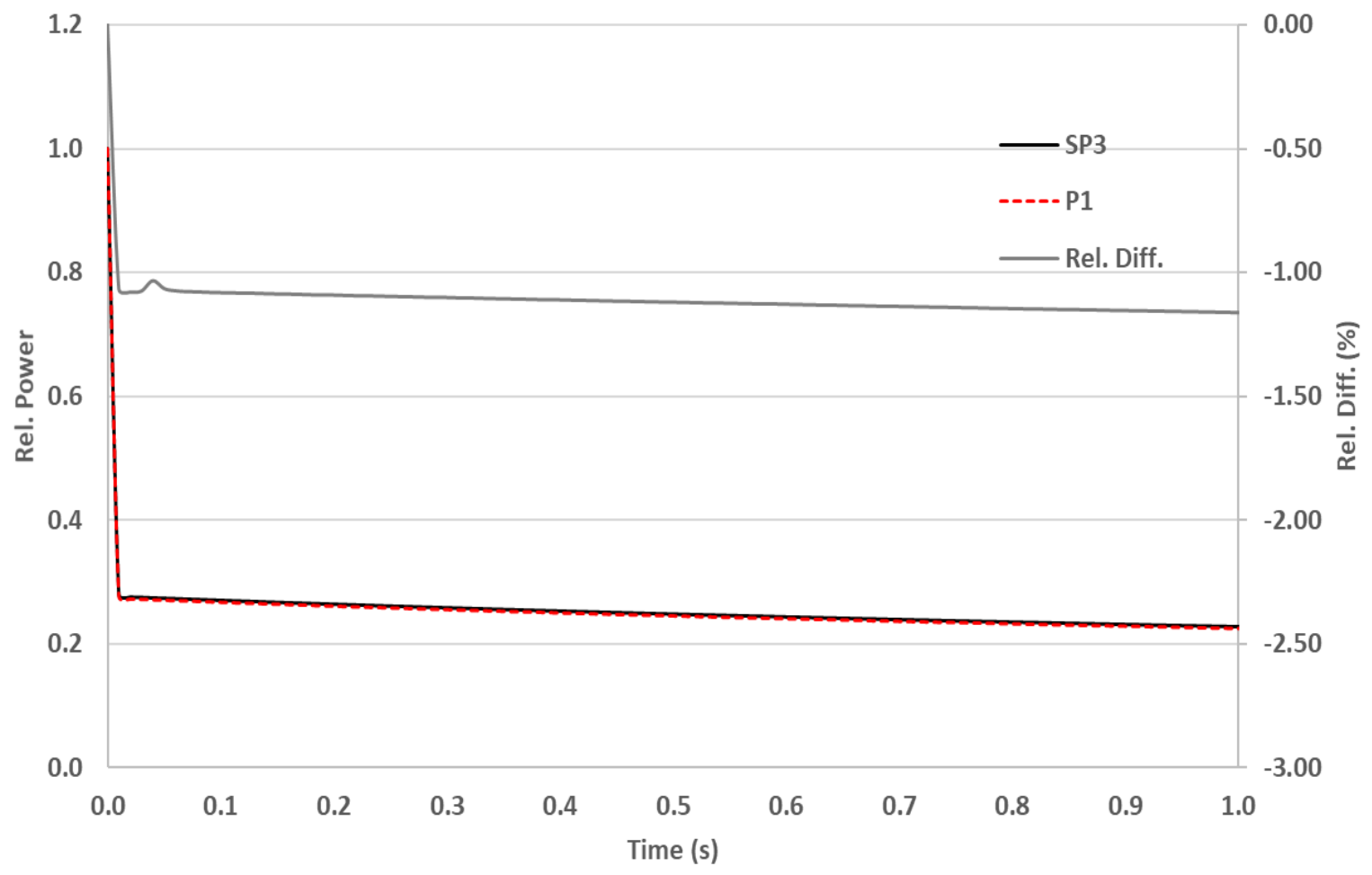

Fig. 3.8. Power comparison after the negative step reactivity insertion for the stationary fuel.

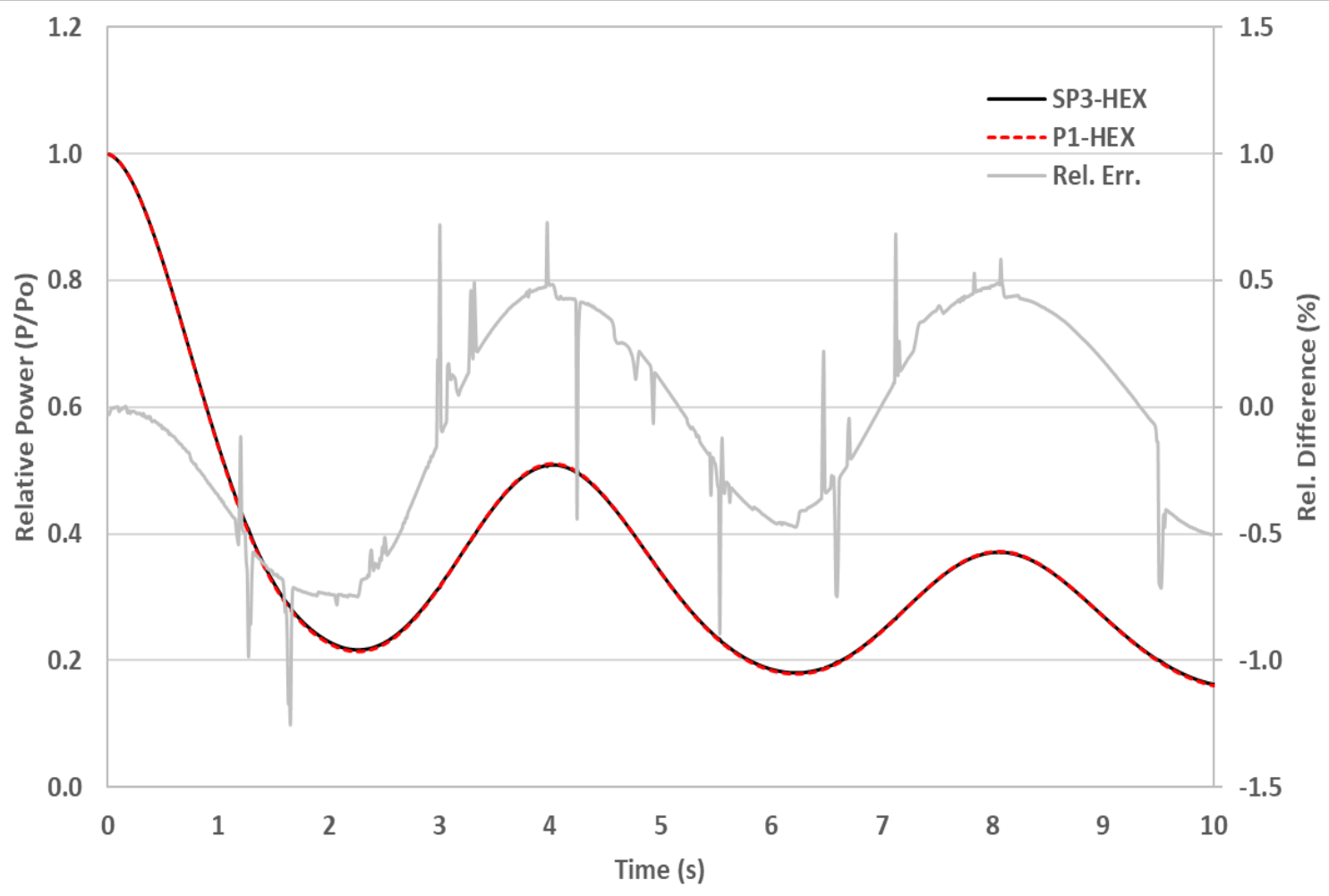

Fig. 3.9. Power comparison after the step increase in the fuel velocity. 


\subsubsection{Verification Tests with Thermal Feedback}

The performance of $\mathrm{P}_{1}$ solver with thermal feedback was verified by solving the steady state problem and various transient scenarios of the MSFR benchmark problem [19]. The standalone thermal-hydraulics module was used for the thermal feedback calculation. The results of three solvers were compared: $\mathrm{SP}_{3}$ solver for hexagonal geometry, $\mathrm{P}_{1}$ solver for hexagonal geometry, and $\mathrm{P}_{1}$ solver for R-Z geometry. A hexagonal geometry core model of six rings of $22.5 \mathrm{~cm}$-pitch hexagons was used.

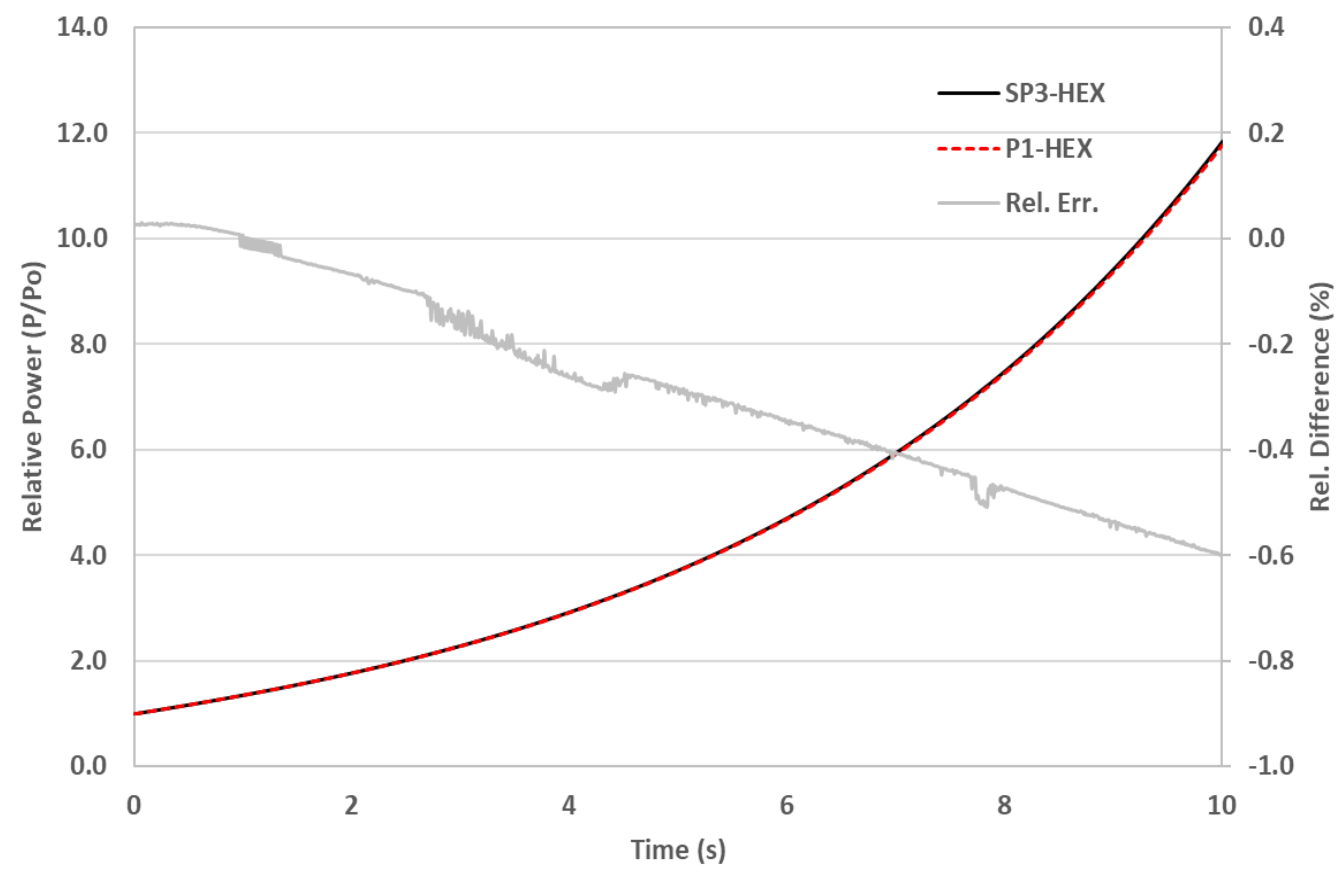

Fig. 3.10. Power comparison after the step decrease in the fuel velocity.

Table 3.3 compares the eigenvalue results of three solvers for the steady state MSFR benchmark problem. The results without thermal feedback obtained with the core-averaged temperature are also included for comparison. As already shown in Table 2.3, a hexagonal geometry core model with large hexagons underestimates the eigenvalue by $100 \mathrm{pcm}$ due to the geometrical approximation. The transport effect partially recovered by the $\mathrm{SP}_{3}$ solver is about $+50 \mathrm{pcm}$. The thermal feedback effect is almost the same for all three solvers with a value of about $85 \mathrm{pcm}$. Although not included in this report, the power density, temperature, pressure, fuel density, and velocity solutions obtained with the three solvers agreed very well with each other.

Computational time was also compared although it is not consistent because of the nonnegligible errors introduced by the approximate hexagonal geometry model. However, this comparison would provide a useful information since the selected hexagonal geometry model with a relatively large hexagon size would be a practical one to avoid a huge computational 
burden. It can be seen that relative to the $\mathrm{SP}_{3}$ solver for hexagonal geometry, the $\mathrm{P}_{1}$ solver for $\mathrm{R}-\mathrm{Z}$ geometry reduces the computational time by $~ 53$ times for the problem without thermal feedback and 8 times for that with thermal feedback with a marginal eigenvalue difference of $\sim 50 \mathrm{pcm}$.

Table 3.3. Eigenvalues of Steady State MSFR Benchmark Problem

\begin{tabular}{|c|c|c|c|c|c|}
\hline \multicolumn{6}{|c|}{ Without Thermal Feedback } \\
\hline & $\begin{array}{l}\text { No. of } \\
\text { Iterations }\end{array}$ & $\begin{array}{l}\text { Calculation } \\
\text { Time (s) }\end{array}$ & Time Ratio & k-eff & a) Diff. (pcm) \\
\hline $\mathrm{SP}_{3}-\mathrm{Hex}$ & 32 & 30.69 & 52.9 & 0.979492 & $-53.9^{b)}(+51.8)$ \\
\hline $\mathrm{P}_{1}$-Hex & 17 & 10.82 & 18.7 & 0.978995 & -105.7 \\
\hline $\mathrm{P}_{1}-\mathrm{RZ}$ & 15 & 0.58 & 1.0 & 0.980009 & - \\
\hline \multicolumn{6}{|c|}{ With Thermal Feedback } \\
\hline & $\begin{array}{l}\text { No. of } \\
\text { Iterations }\end{array}$ & $\begin{array}{l}\text { Calculation } \\
\text { Time (s) }\end{array}$ & Time Ratio & k-eff & ${ }^{\mathrm{c})}$ Diff. (pcm) \\
\hline $\mathrm{SP}_{3}-\mathrm{Hex}$ & 42 & 42.01 & 8.0 & 0.980351 & 85.9 \\
\hline$\overline{\mathrm{P}_{1}-\mathrm{Hex}}$ & 19 & 19.83 & 3.8 & 0.979839 & 84.4 \\
\hline $\mathrm{P}_{1}-\mathrm{RZ}$ & 15 & 5.24 & 1.0 & 0.980213 & 86.8 \\
\hline
\end{tabular}

a) Difference in eigenvalue from the $\mathrm{P}_{1}$-RZ result

b) Difference in eigenvalue from the $\mathrm{P}_{1}$-Hex result

c) Difference of eigenvalue with thermal feedback from that without thermal feedback

For transient problems, unprotected transient overpower (UTOP), unprotected pump over speed (UPOS), unprotected loss of flow (ULOF), unprotected loss of heat sink (ULOHS), unprotected fuel salt overcooled (UFSOC) scenarios were solved using the three solvers with the same geometries used for the steady state problem. The description of each transient and the simulation methodology can be found in $[9,25,26]$. For each transient case, the power and core-averaged fuel salt temperature changes with time are compared in Fig. 3.11 through Fig. 3.22. It can be seen that despite the differences in geometrical models and transport approximations (diffusion vs. $\mathrm{SP}_{3}$ ), the differences in power and fuel salt temperature of the three solutions are small. The maximum difference in power of the two $\mathrm{P}_{1}$ solutions from the $\mathrm{SP}_{3}$ solution is less than $0.9 \%$ for all the transients except for the super-prompt critical UTOP of a reactivity insertion of $200 \mathrm{pcm}$. In the super-prompt critical UTOP, the maximum difference is $2.0 \%$ for the $\mathrm{P}_{1}$ solver with $\mathrm{R}-\mathrm{Z}$ geometry and $5.0 \%$ for the $\mathrm{P}_{1}$ solver with hexagonal geometry. The maximum difference in the core-averaged fuel salt temperature is less than $0.8 \%$ for all scenarios. With these comparable accuracies, the $\mathrm{P}_{1}$ solver for $\mathrm{R}-\mathrm{Z}$ geometry reduces the computational time about 10 to 60 times relative to the $\mathrm{SP}_{3}$ solver and about 4.5 to 9.5 times relative to the $\mathrm{P}_{1}$ solver for hexagonal geometry. The solution accuracy 
and computational efficiency of three solvers are compared in Table 3.4 for all the six transient scenarios.

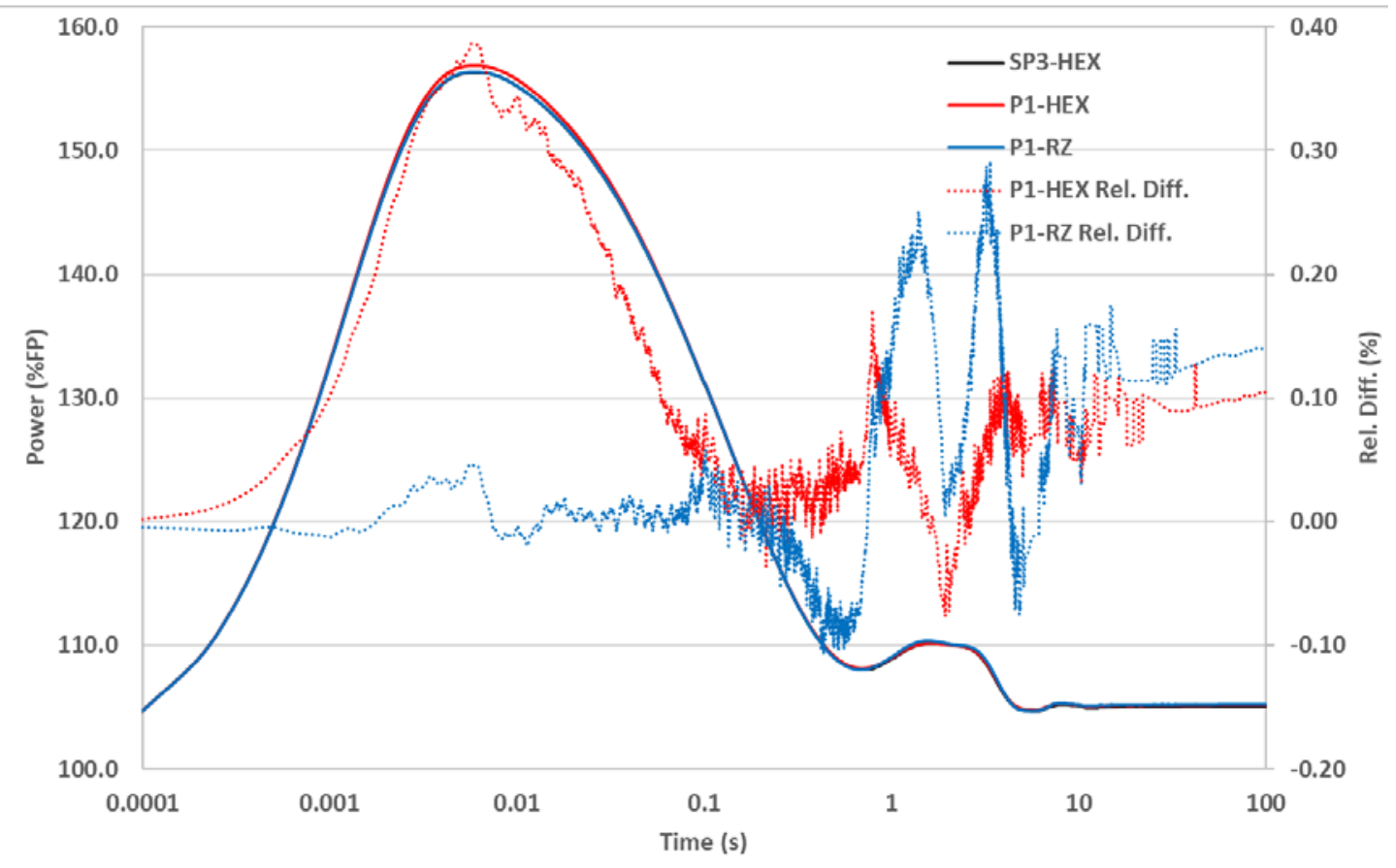

Fig. 3.11. Power evolution during the UTOP transient with $50 \mathrm{pcm}$ reactivity insertion.

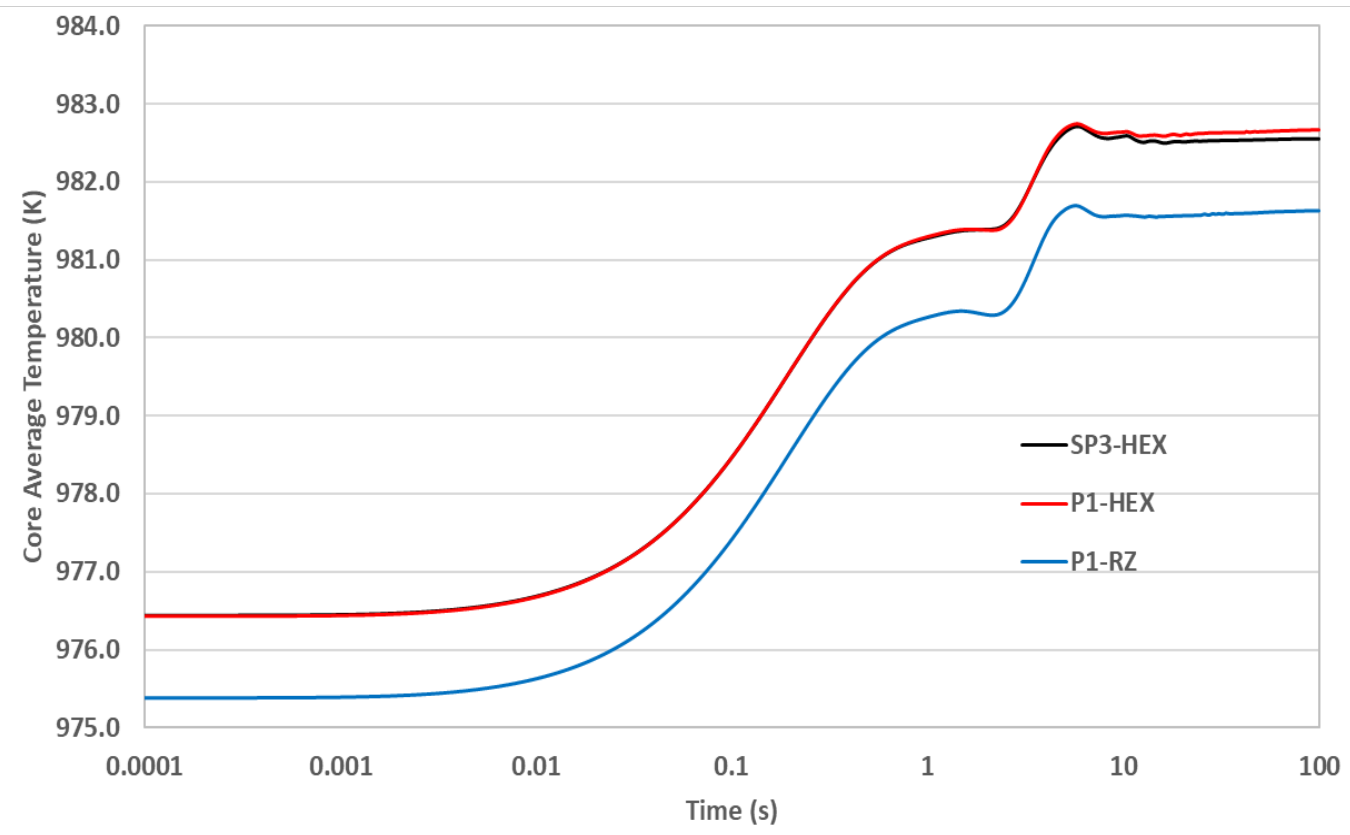

Fig. 3.12. Core average temperature rise across core during the UTOP transient with $50 \mathrm{pcm}$ reactivity insertion. 
Multiphysics Coupling of PROTEUS-NODAL and SAM for Molten Salt Reactor Simulation February 28, 2020

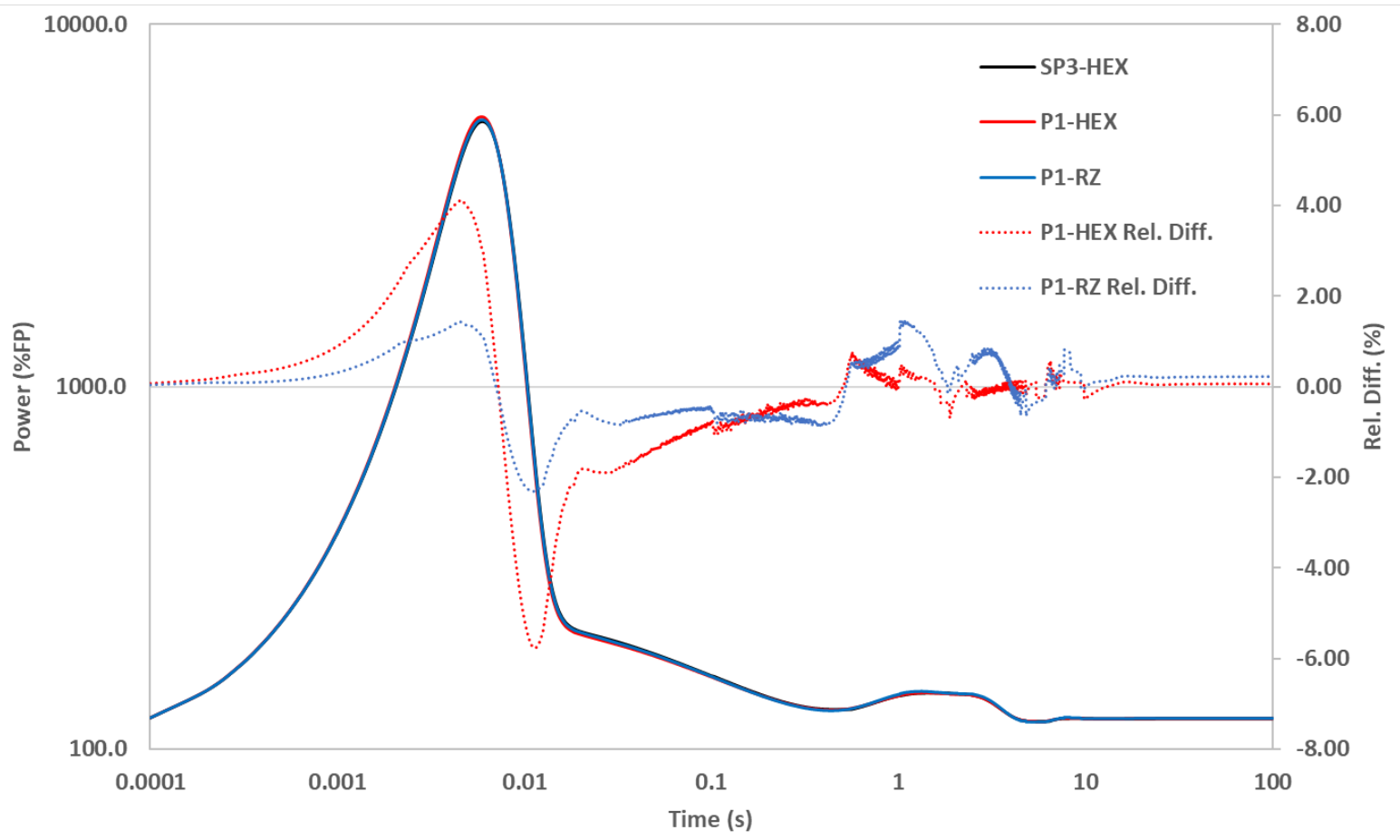

Fig. 3.13. Power evolution during the UTOP transient with $200 \mathrm{pcm}$ reactivity insertion.

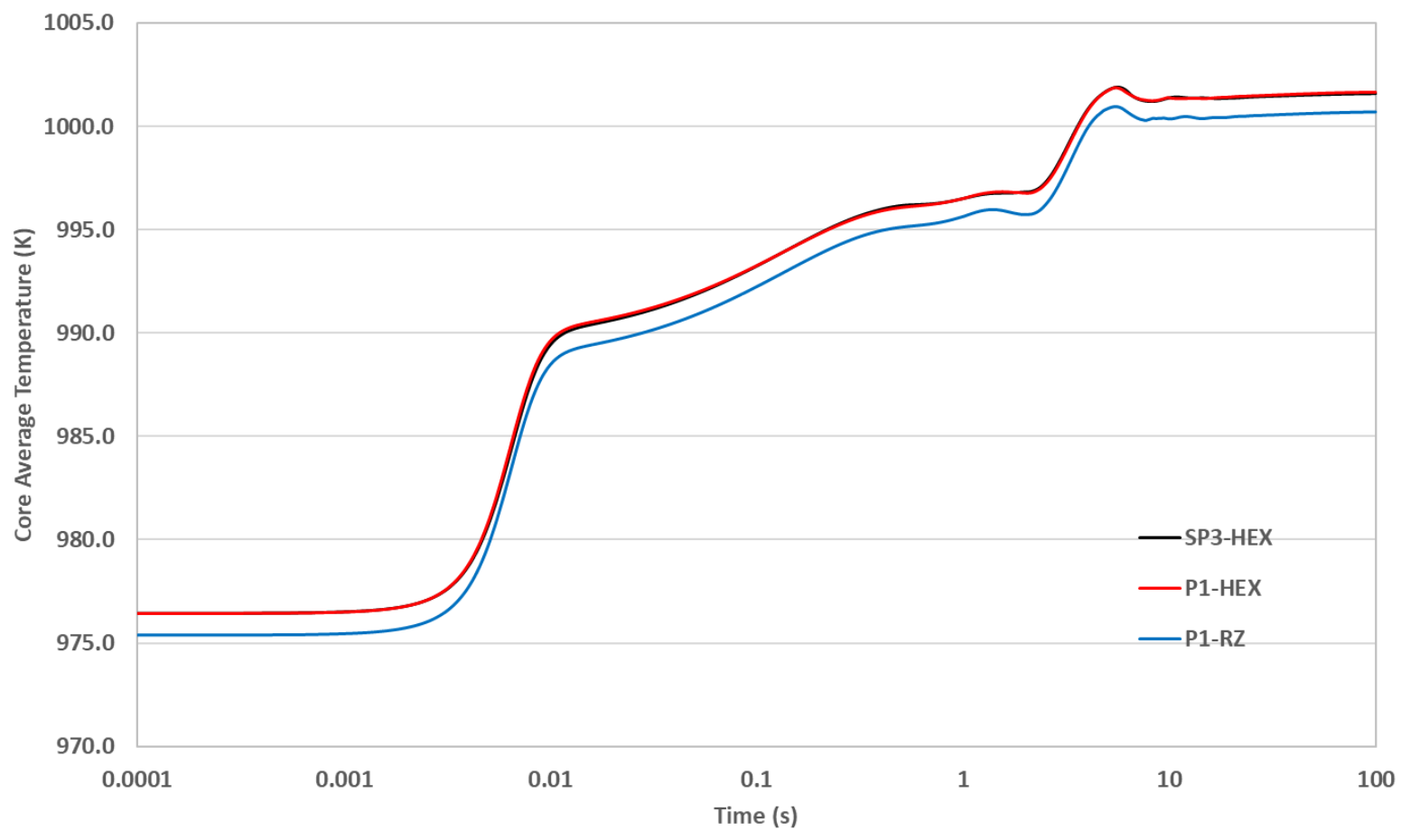

Fig. 3.14. Core average temperature rise across core during the UTOP transient with $200 \mathrm{pcm}$ reactivity insertion. 


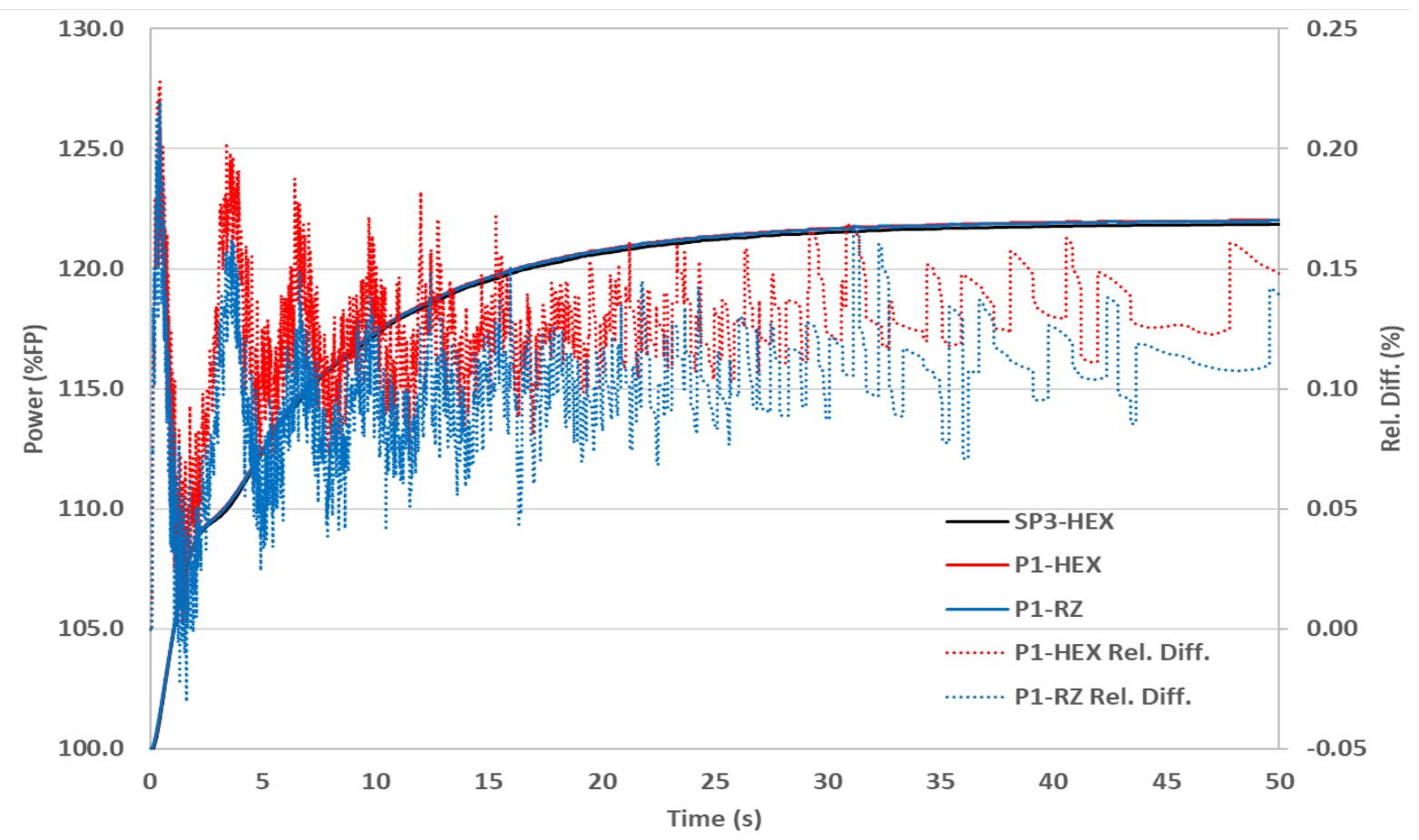

Fig. 3.15. Power evolution during the UPOS transient.

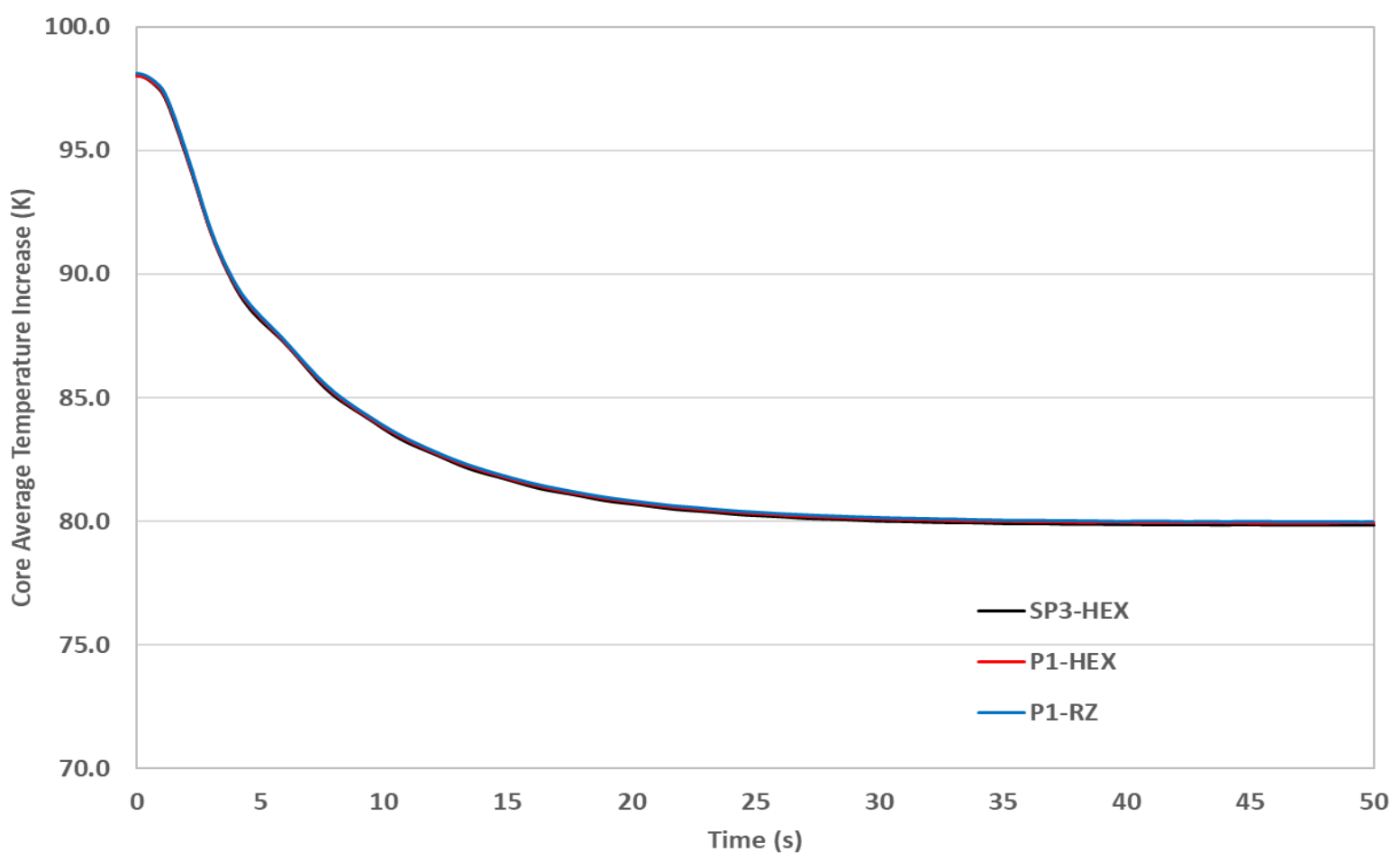

Fig. 3.16. Average temperature rise of fuel salt across core during the UPOS transient. 
Multiphysics Coupling of PROTEUS-NODAL and SAM for Molten Salt Reactor Simulation February 28, 2020

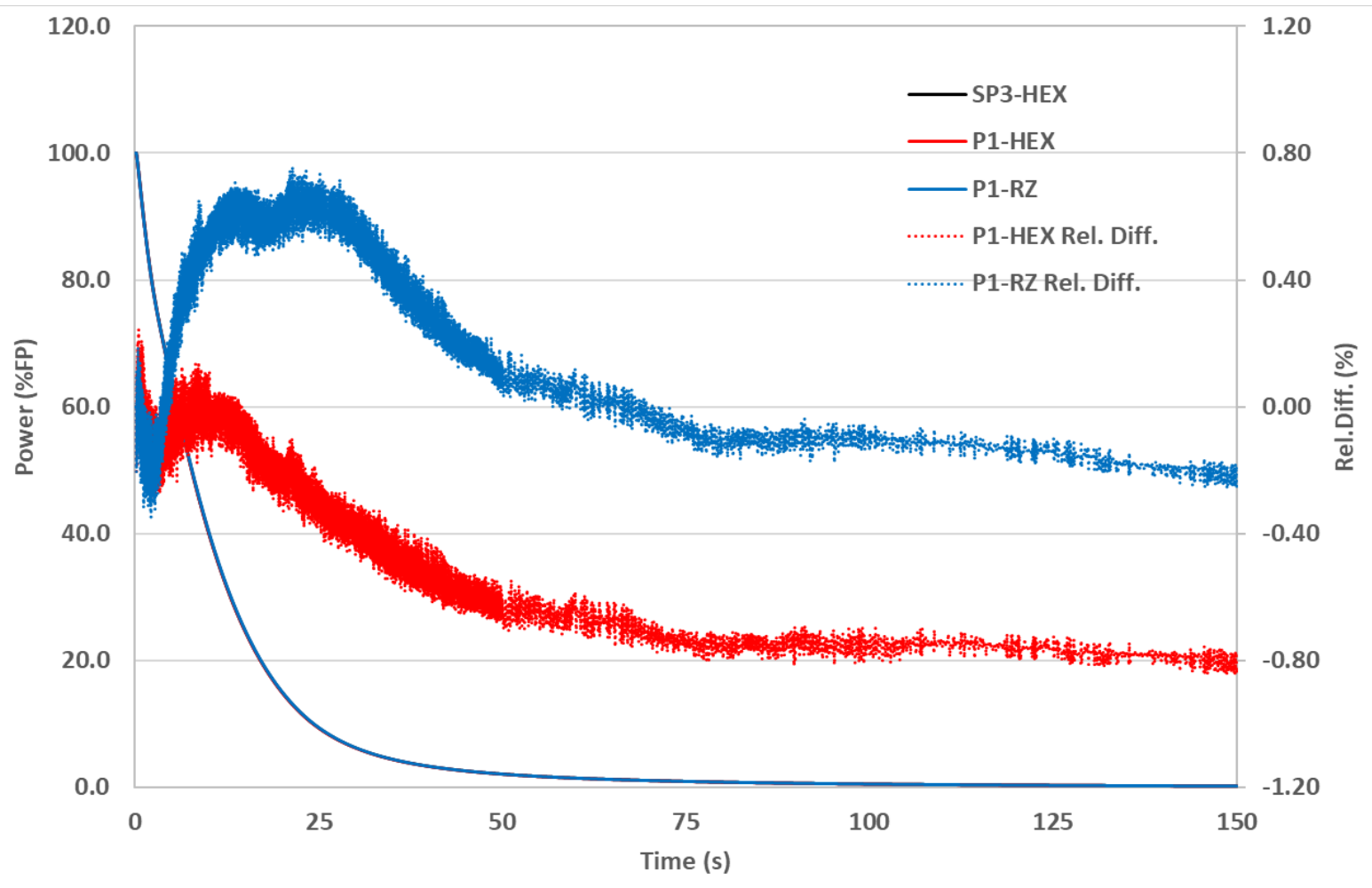

Fig. 3.17. Power evolution during the ULOF transient.

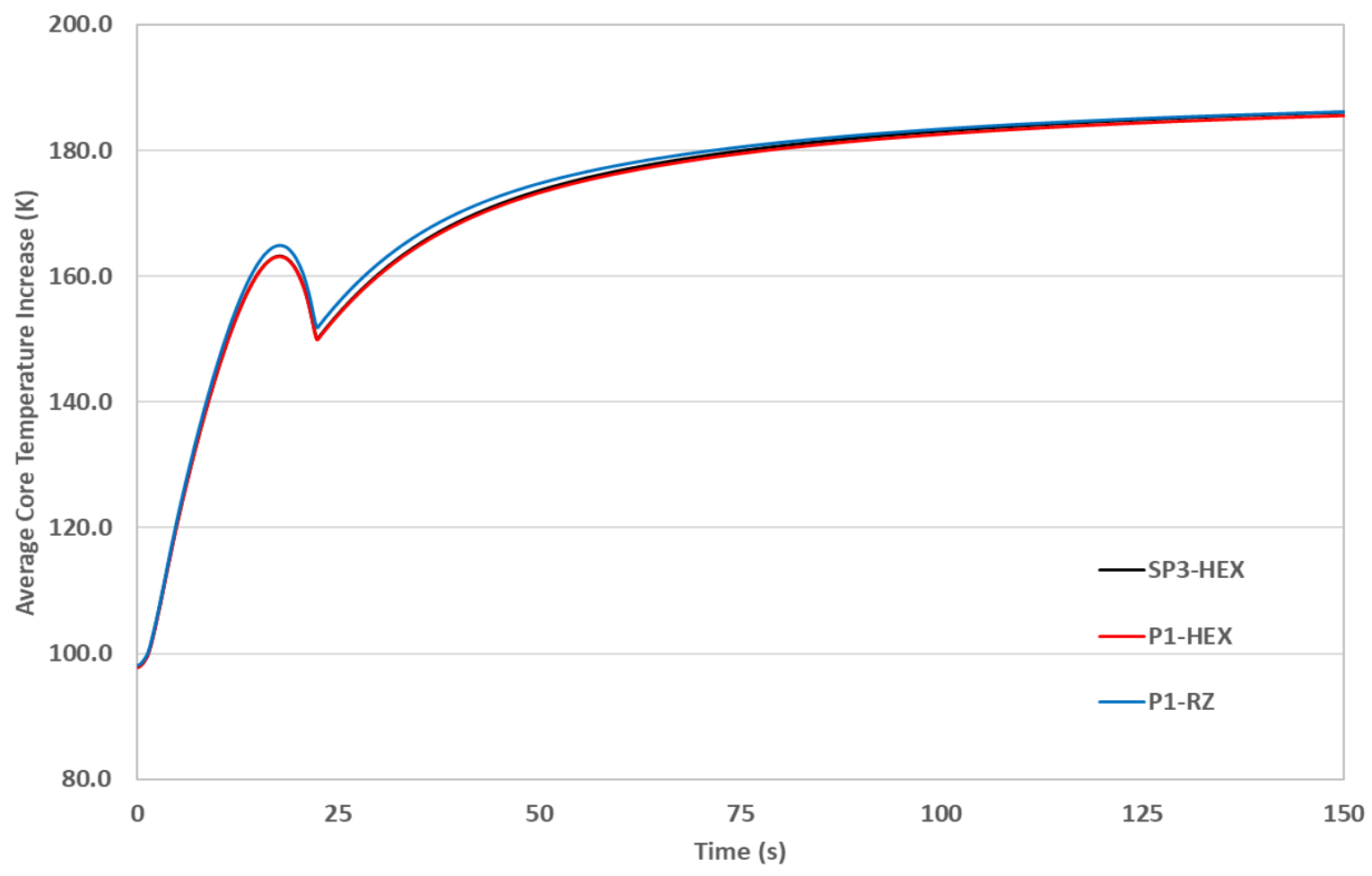

Fig. 3.18. Average temperature rise of fuel salt across core during the ULOF transient. 


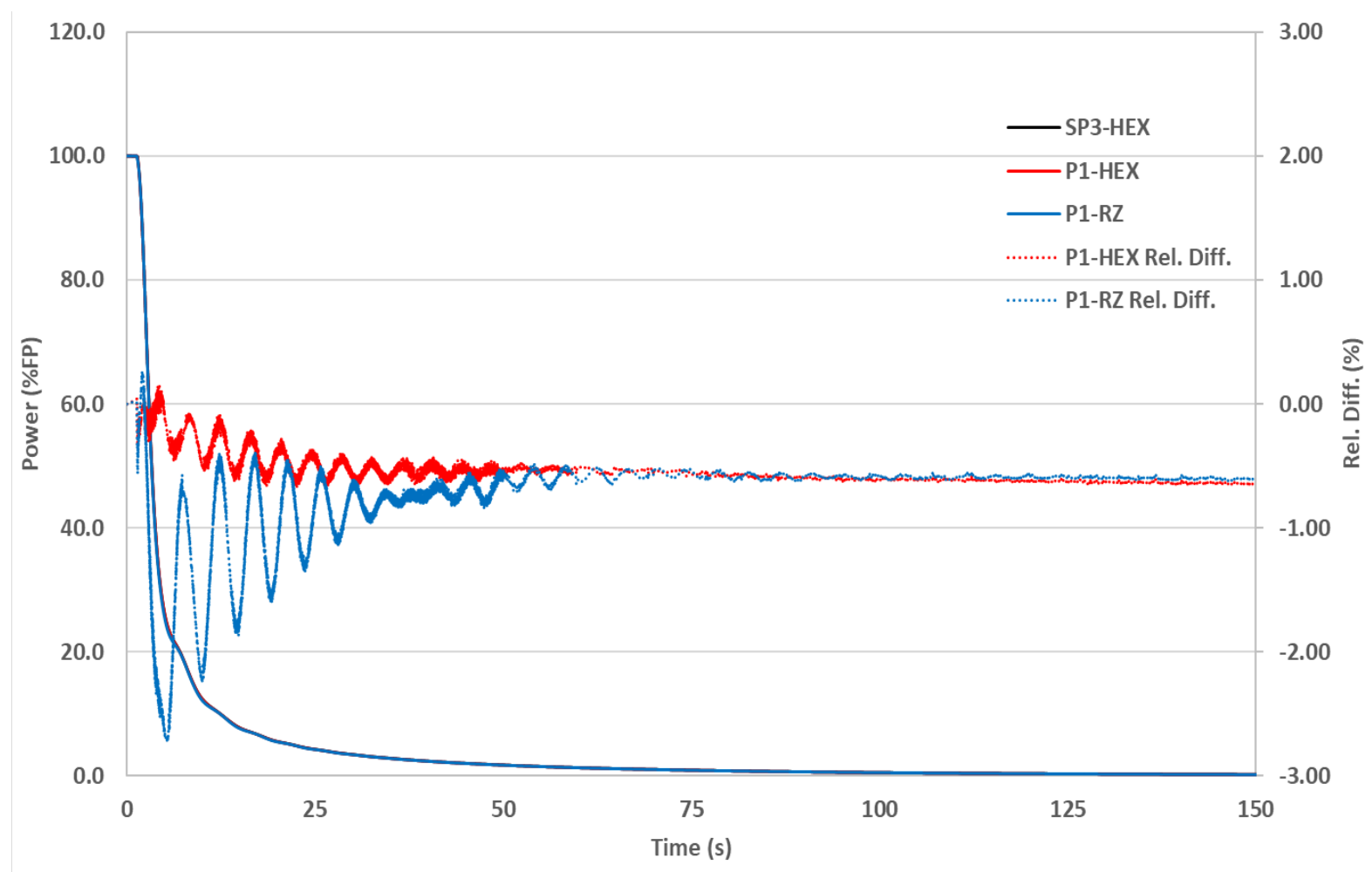

Fig. 3.19. Power evolution during the ULOHS transient.

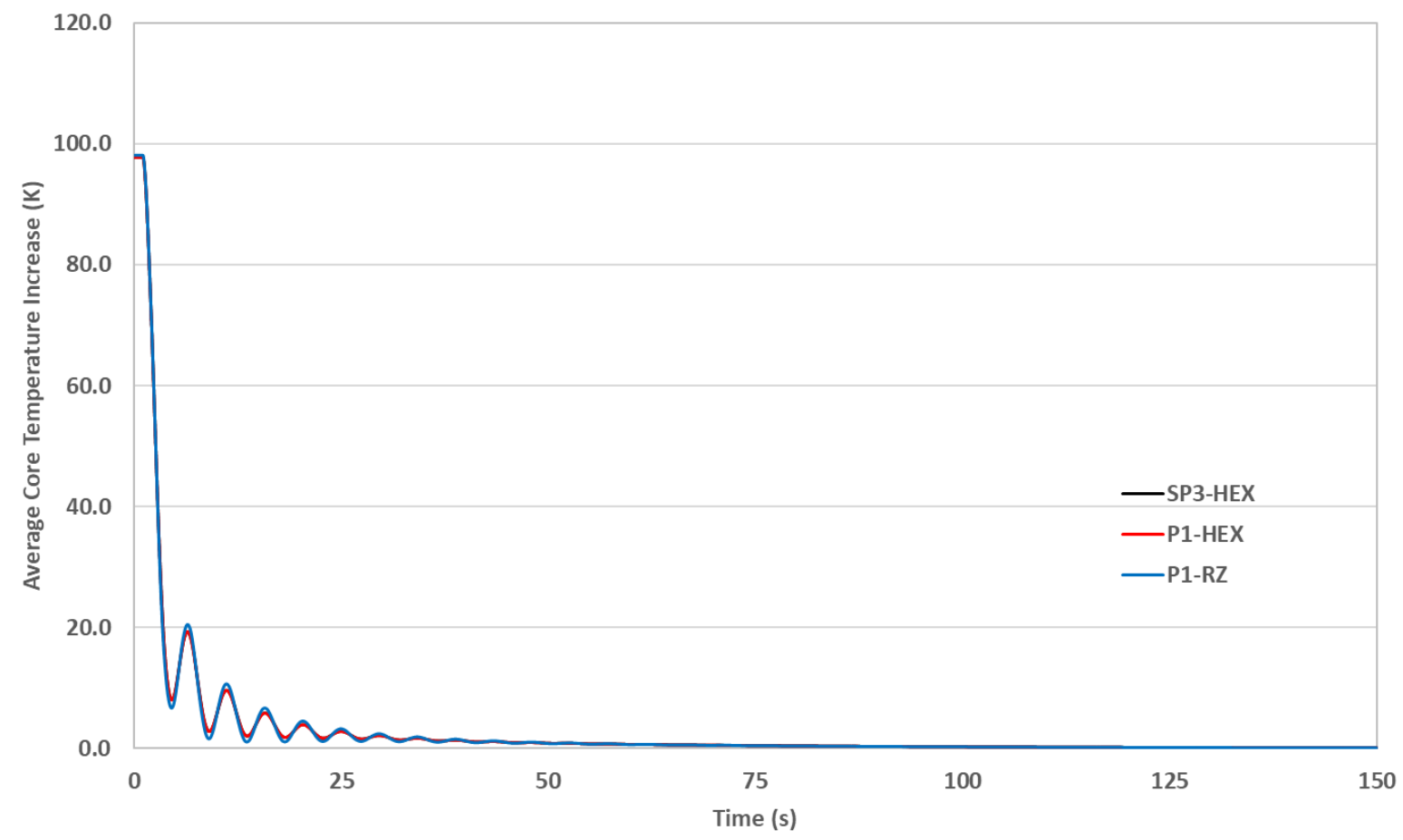

Fig. 3.20. Average temperature rise of fuel salt across core during the ULOHS transient. 
Multiphysics Coupling of PROTEUS-NODAL and SAM for Molten Salt Reactor Simulation February 28, 2020

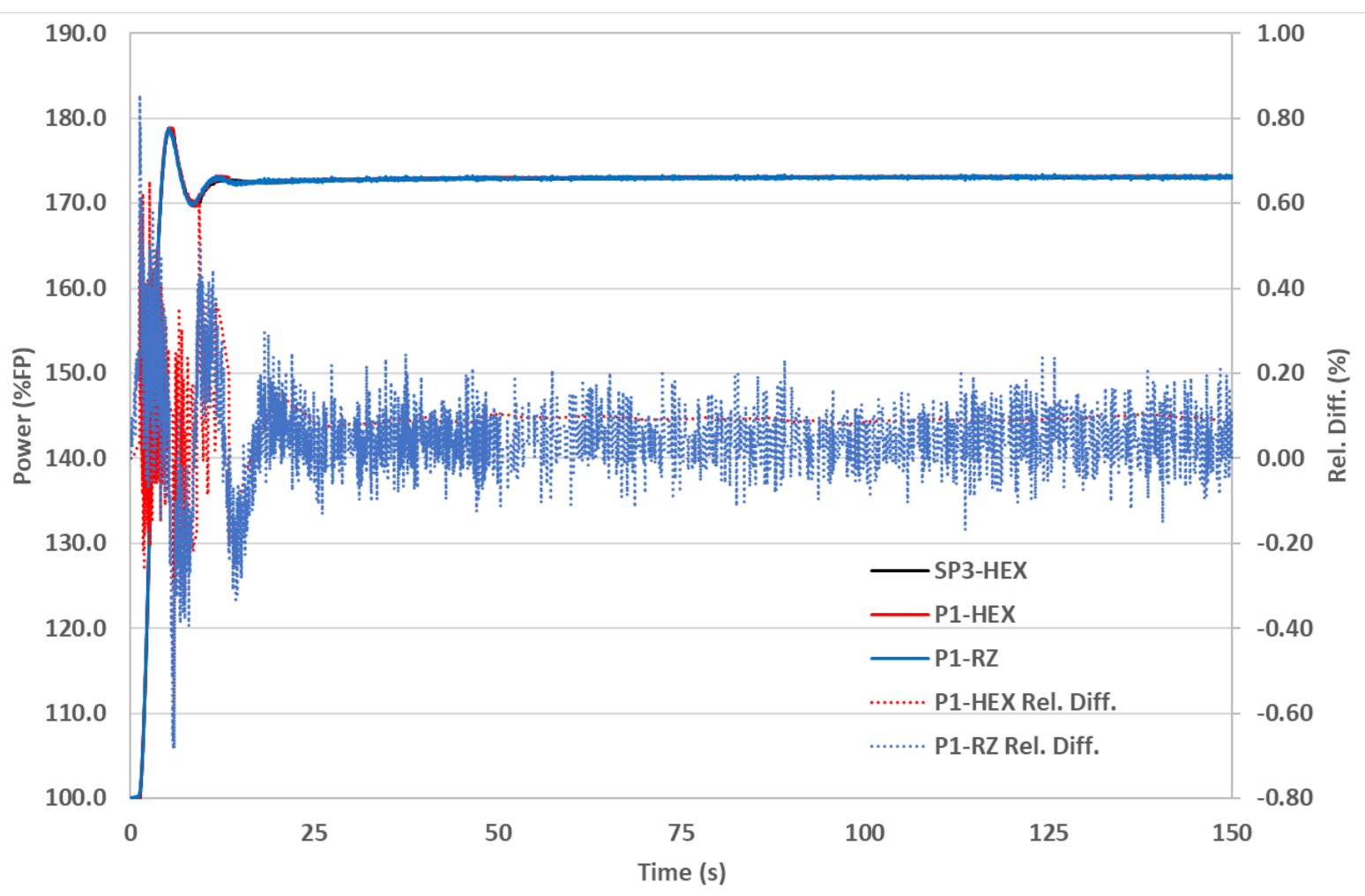

Fig. 3.21. Power evolution during the UFSOC transient.

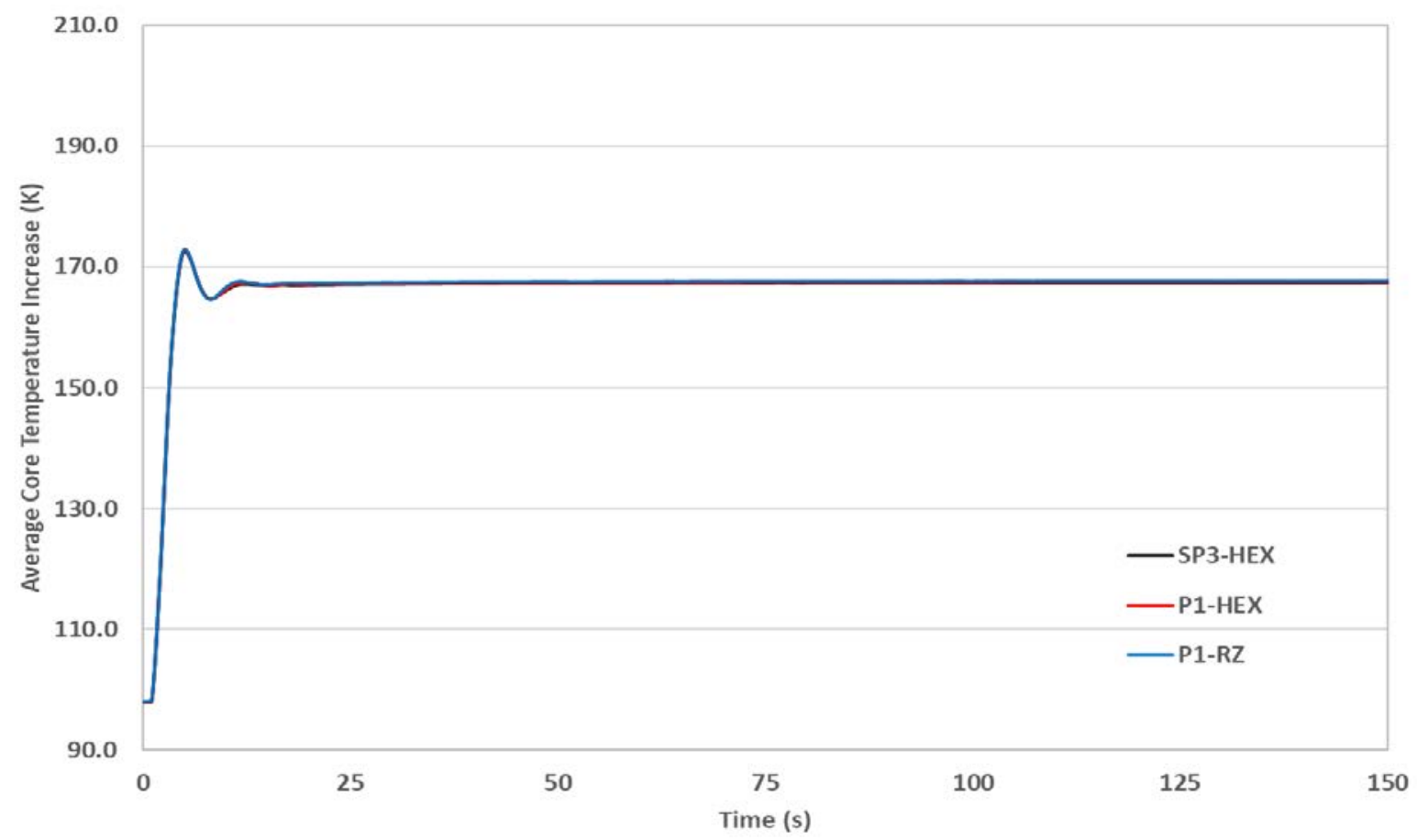

Fig. 3.22. Average temperature rise of fuel salt across core during the UFSOC transient. 
Table 3.4. Performance Comparison of Three Solvers for MSFR Transient Problems

\begin{tabular}{|c|c|c|c|c|c|c|}
\hline \multirow{2}{*}{$\begin{array}{l}\text { Transient } \\
\text { Scenario }\end{array}$} & \multirow{2}{*}{$\begin{array}{l}\text { No. Time } \\
\text { Steps }\end{array}$} & \multirow{2}{*}{$\begin{array}{l}\text { End Time } \\
\text { (sec) }\end{array}$} & \multicolumn{3}{|c|}{ Max. No. of Iterations } & \\
\hline & & & $\mathrm{SP}_{3}$-Hex & $\mathrm{P}_{1}$-Hex & $\mathrm{P}_{1}-\mathrm{RZ}$ & \\
\hline UTOP-50 & 4600 & 100 & 451 & 10 & 10 & \\
\hline UTOP-200 & 11800 & 100 & 442 & 9 & 9 & \\
\hline UPOS & 5000 & 50 & 142 & 4 & 3 & \\
\hline ULOF & 7000 & 150 & 108 & 3 & 3 & \\
\hline UFSOC & 8000 & 200 & 82 & 3 & 5 & \\
\hline ULOHS & 7000 & 150 & 323 & 3 & 3 & \\
\hline \multirow{2}{*}{$\begin{array}{l}\text { Transient } \\
\text { Scenario } \\
\end{array}$} & \multicolumn{3}{|c|}{ Computational Time (hr) } & \multicolumn{3}{|c|}{ Time Ratio } \\
\hline & $\mathrm{SP}_{3}$-Hex & $\mathrm{P}_{1}$-Hex & $\mathrm{P}_{1}-\mathrm{RZ}$ & $\mathrm{SP}_{3}$-Hex & $\mathrm{P}_{1}$-Hex & $\mathrm{P}_{1}-\mathrm{RZ}$ \\
\hline UTOP-50 & 29.93 & 2.75 & 0.449 & 66.70 & 6.12 & 1.0 \\
\hline UTOP-200 & 22.80 & 4.79 & 1.106 & 20.61 & 4.33 & 1.0 \\
\hline UPOS & 25.48 & 4.22 & 0.460 & 55.43 & 9.17 & 1.0 \\
\hline ULOF & 8.83 & 4.40 & 0.742 & 11.91 & 5.93 & 1.0 \\
\hline UFSOC & 9.32 & 4.78 & 0.498 & 18.72 & 9.60 & 1.0 \\
\hline ULOHS & 21.85 & 4.10 & 0.726 & 30.10 & 5.65 & 1.0 \\
\hline \multirow{2}{*}{$\begin{array}{l}\text { Transient } \\
\text { Scenario }\end{array}$} & \multicolumn{3}{|c|}{ Max. Power Diff. (\%) } & \multicolumn{3}{|c|}{ Max. Core Avg. Temperature Diff. (\%) } \\
\hline & $\mathrm{SP}_{3}$-Hex & $\mathrm{P}_{1}$-Hex & $\mathrm{P}_{1}-\mathrm{RZ}$ & $\mathrm{SP}_{3}$-Hex & $\mathrm{P}_{1}$-Hex & $\mathrm{P}_{1}-\mathrm{RZ}$ \\
\hline UTOP-50 & - & 0.386 & 0.291 & - & 0.012 & 0.113 \\
\hline UTOP-200 & - & 5.772 & 2.312 & - & 0.028 & 0.110 \\
\hline UPOS & - & 0.228 & 0.220 & - & 0.017 & 0.014 \\
\hline ULOF & - & 0.844 & 0.054 & - & 0.756 & 0.119 \\
\hline UFSOC & - & 0.651 & 0.854 & - & 0.028 & 0.030 \\
\hline ULOHS & - & 0.667 & 2.719 & - & 0.029 & 0.135 \\
\hline
\end{tabular}




\section{Coupling of PROTEUS-NODAL and SAM}

In a liquid fuel MSR, the core reactivity is directly related to the speed of flowing fuel because of the precursors that decay outside the core and the thermal expansion of the liquid fuel introduces large negative reactivity feedback. As a result, the neutronics and thermalhydraulics are strongly coupled. In order to enhance the thermal-hydraulics modeling capabilities by overcoming the limitation of the standalone thermal-hydraulics solver, PROTEUS-NODAL has been coupled with the system analysis code SAM [11] under the MOOSE framework [12]. Since PROTEUS-NODAL is not an application of the MOOSE framework, a MOOSE wrapper named “TreeFrog” was developed. Using the MOOSE framework, a master application named “TreeKangaroo" was developed as well to control the coupling calculations between PROTEUS-NODAL and SAM. At the moment, the coupling is limited to the $1 \mathrm{D}$ core models of SAM. The coupled calculation of PROTEUSNODAL and SAM was first verified by comparing the steady state analysis results with those obtained by manual iteration between PROTEUS-NODAL and the computational fluid dynamics (CFD) code ANSYS CFX [14]. Then the MSFR transient analysis results of the coupled system of PROTEUS-NODAL and SAM were compared with the other solutions found in the open literature.

The coupling calculation scheme of PROTEUS-NODAL and SAM is discussed first. This is followed by the verification test results of the SAM code with the ANSYS CFX code for the steady state MSFR problem. Then the MSFR transient analysis results of the coupled system of PROTEUS-NODAL and SAM are compared with those of other code systems. The descriptions of TreeFrog and TreeKangaroo are provided in Appendix E along with sample input files.

\subsection{Coupled Calculation Scheme}

Since PROTEUS-NODAL is not an application of the MOOSE framework, the mesh system and associated variables used in PROTEUS-NODAL cannot be directly communicated with other codes under the MOOSE framework. Thus, the MOOSE application called TreeFrog was developed as the wrapper for the PROTEUS-NODAL code. The communications between different MOOSE applications rely on the MOOSE "MultiApps" and “Transfer" systems. In the MOOSE framework, multiple independent applications can be defined in a tree-like multi-level structure, as shown in Fig. 4.1. In the MultiApps system, a master application controls its sub-applications. In the coupled system of PROTEUS-NODAL and SAM, TreeFrog (i.e., the MOOSE wrapper of PROTEUSNODAL) and SAM are independently run as two sub-applications controlled by a master 
application called TreeKangaroo. The Transfer system provides the functions to transfer the solution data between applications in the MultiApp system. The Transfer system consists of three main types of transfers: field mapping, post-processed spatial data, and scalar values [12]. In this work, the data transfer functions between PROTEUS-NODAL and SAM are based on the transfer of post-processed spatial data.

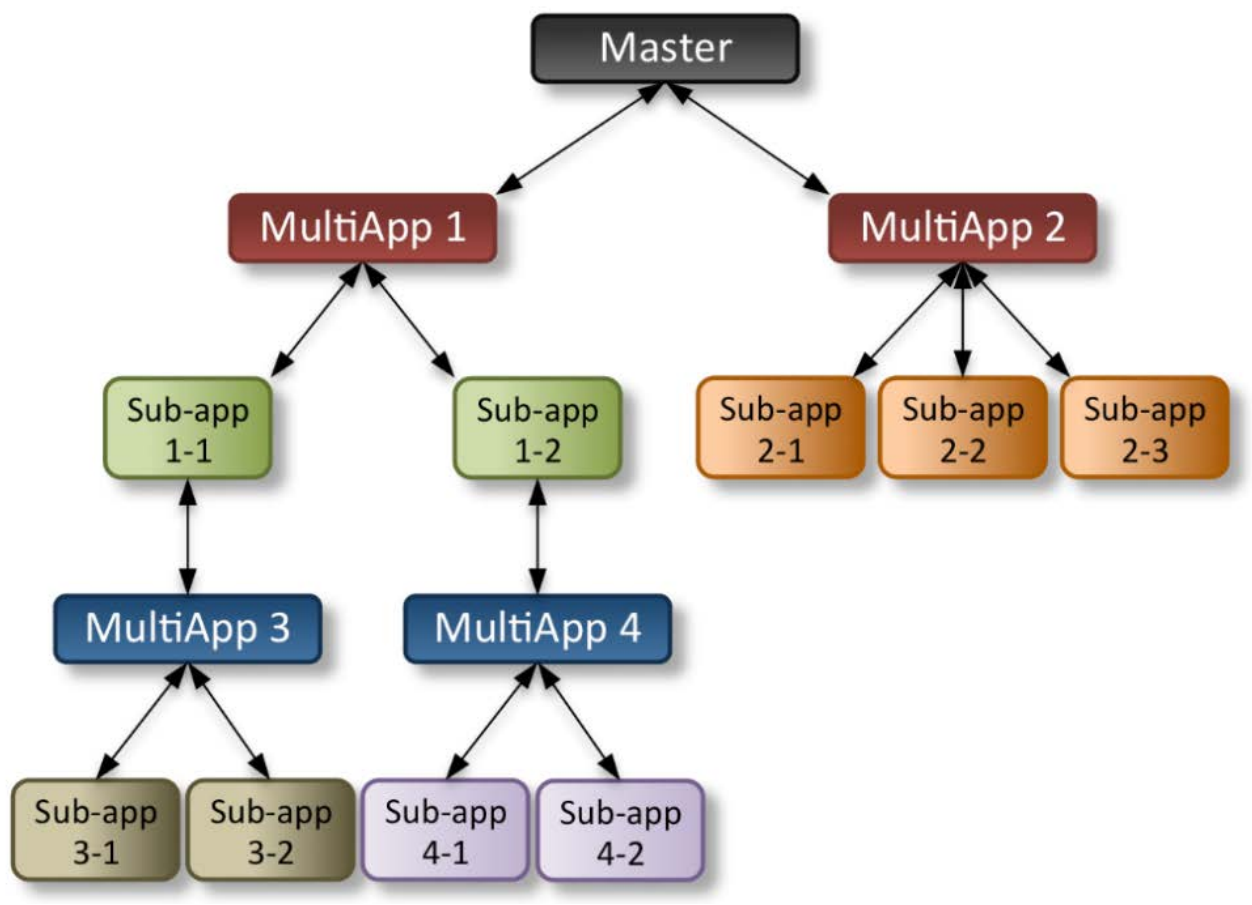

Fig. 4.1. MOOSE MultiApps system [12].

\subsubsection{PROTEUS-NODAL Wrapper TreeFrog}

The PROTEUS-NODAL code is wrapped inside TreeFrog with the "ExternalProblem" class of the MOOSE framework. This class contains mainly two methods: one for an executioner and the other for a data transferrer. The executioner runs PROTEUS-NODAL, while the data transferrer transfers data between PROTEUS-NODAL and TreeFrog. The MOOSE-based TreeFrog mesh is defined to store data to be passed from and to PROTEUSNODAL such as power density, temperature, density, and velocity of the fuel salt. TreeFrog communicates with PROTEUS-NODAL before and after running PROTEUS-NODAL at each time step to ensure that the data are up to date.

PROTEUS-NODAL is compiled as a dynamic library such that the subroutines of PROTEUS-NODAL can be accessed from TreeFrog. At the beginning of a calculation, TreeFrog calls the subroutines that read the PROTEUS-NODAL input files and initializes the 
relevant variables. Then the executioner runs PROTEUS-NODAL at each time step in a transient simulation or at each Picard iteration in a steady state simulation. The memory is freed at the final step of the TreeFrog run.

To facilitate the data transfer between TreeFrog and SAM, the axial mesh size of TreeFrog is set to equal to that of the SAM channel. The variables in the TreeFrog meshes and those in the nodes of PROTEUS-NODAL are transferred with appropriate interpolation and integration. The power densities in TreeFrog meshes are obtained by integrating the intra-nodal power density of the corresponding node of the PROTEUS-NODAL code over the axial direction. In both the $\mathrm{SP}_{3}$ and $\mathrm{P}_{1}$ solvers, the intra-nodal flux is represented by polynomials of a specified order as

$$
\phi_{g}(\xi)=\sum_{j=0}^{n_{z}} a_{g, j} f_{j}(\xi), \quad \xi=2 \frac{z-z_{\text {in }}}{z_{\text {out }}-z_{\text {in }}}-1
$$

where $z_{\text {in }}$ and $z_{\text {out }}$ are the lower and upper coordinates of the node. In the $\mathrm{SP}_{3}$ solver, $n_{z}$ is 2 and $f_{j}(\xi)$ is the Legendre polynomial of order $j$. For the $\mathrm{P}_{1}$ solver, $n_{z}$ is the user-specified order, $a_{g, j}$ corresponds to $\zeta_{g, j}$ in Eq. (2.3), and $f_{j}(\xi)$ is obtained by radially averaging the basis function $f_{j}(\boldsymbol{r})$ in Eq. (2.3). With the axial nodes of PROTEUS-NODAL and the axial meshes of TreeFrog shown in Fig. 4.2, the power density in the $m$-th axial mesh of TreeFrog in the $i$-th channel of SAM is calculated as

$$
P_{i, m}=\frac{\sum_{j=0}^{n_{z}} \int_{\xi_{b}}^{\xi_{u}} b_{j} f_{j}(\xi) d \xi}{\xi_{u}-\xi_{b}}, \quad b_{j}=\sum_{g} a_{g, j} \kappa \Sigma_{f, g},
$$




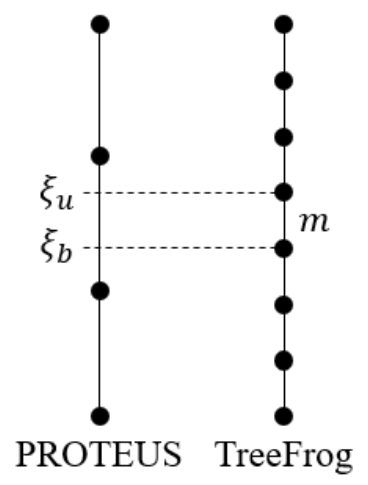

Fig. 4.2. Axial nodes of PROTEUS-NODAL and axial meshes of TreeFrog.

where $\xi_{b}$ and $\xi_{b}$ are the normalized positions for the lower and upper bounds of the $m$-th

TreeFrog axial mesh, respectively. For the hexagonal geometry, $P_{i, m}$ is determined by averaging the power densities of the hexagonal assemblies that belong to the $i$-th channel of SAM.

The average fuel density in a node of PROTEUS-NODAL is evaluated by integrating the fuel densities in the corresponding TreeFrog meshes based on the piece-wise linear interpolation. The average fuel temperature and velocity in a node of PROTEUS-NODAL are obtained with the density-weighted integration of each variable based on a piece-wise linear interpolation.

\subsubsection{Overall Computational Procedure of TreeKangaroo}

TreeKangaroo was developed as the master application to control TreeFrog and SAM as independent sub-applications. Fig. 4.3 shows the structure and the coupling scheme of TreeKangaroo. The data transfer between the TreeFrog and SAM meshes are done through the TreeKangaroo mesh using the MOOSE function "MultiAppCopyTransfer," which supports the transfer of all kinds of variables between two MultiApps if their meshes are identical. 


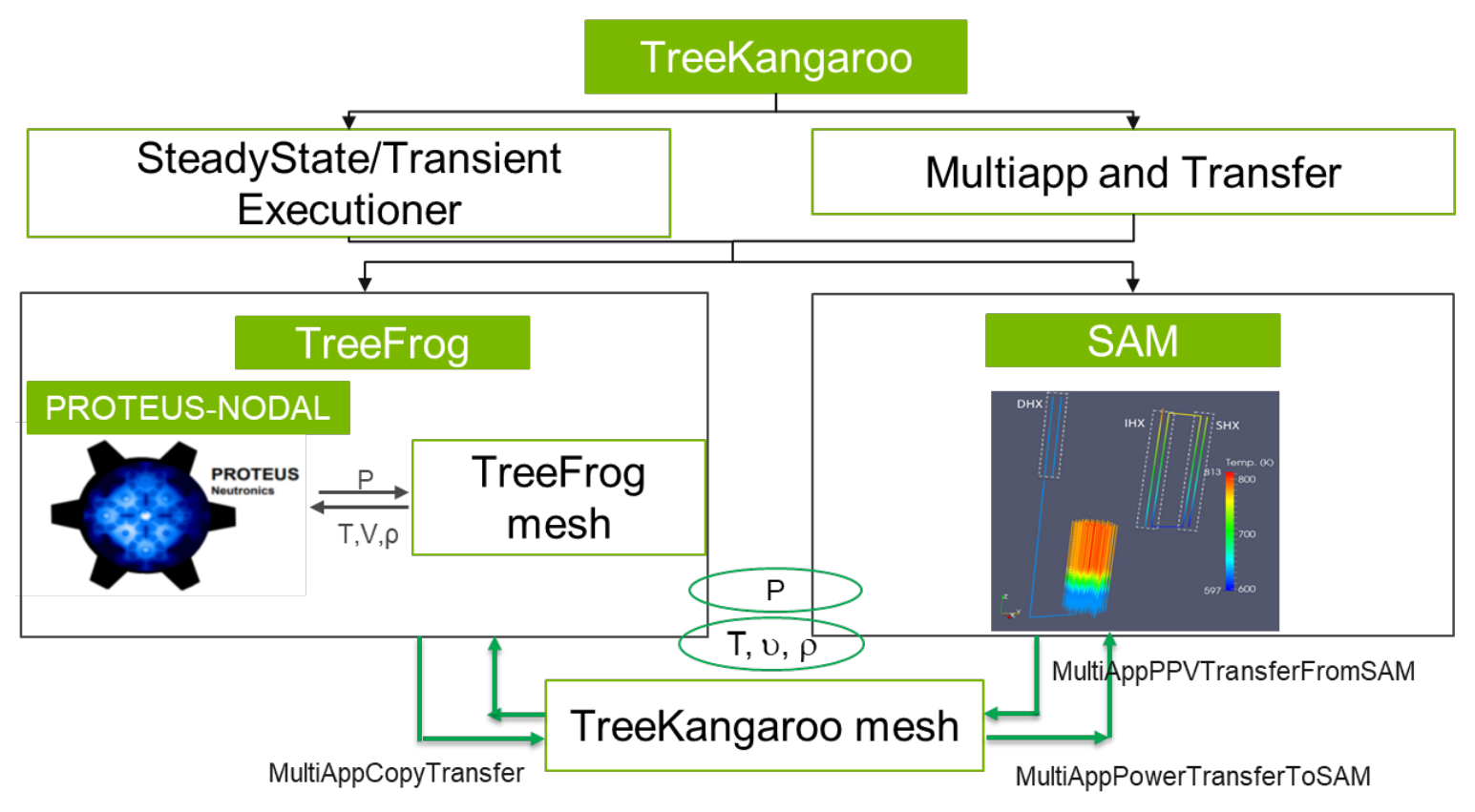

Fig. 4.3. Data flow and coupling scheme between PROTEUS-NODAL and SAM under the MOOSE framework.

Two functions “MultiAppPowerTransferToSam” and "MultiAppVPPTransferFromSam” were implemented in TreeKangaroo for the data transfer between TreeKangaroo and SAM. The function "MultiAppPowerTransferToSam" is used to transfer the power density from TreeKangaroo to SAM. To store the power density transferred from TreeKangaroo, a new heat source object was defined in SAM as an "AuxVariables" of MOOSE. The function MultiAppPowerTransferToSam determines the mapping between the TreeKangaroo mesh and the SAM mesh. The function "MultiAppVPPTransferFromSam" is used to transfer the velocity, temperature, and density data from the SAM mesh to the TreeKangaroo mesh. In SAM, all the velocity, temperature, and density data of a channel are output to one "VectorPostprocessors". In the "MultiAppVPPTransferFromSam" function, the velocity, temperature, and density data are read from a "VectorPostprocessors" of SAM, and the transferred data are stored as “AuxVariables” in the TreeKangaroo mesh.

For steady state calculations, the Picard iteration is used as illustrated in Fig. 4.4. Within each iteration, TreeFrog and SAM are executed sequentially. The PROTEUS-NODAL calculation is performed using the temperature, density, and velocity of SAM in the last Picard iteration. Then the power distribution calculated by PROTEUS-NODAL is mapped to the 


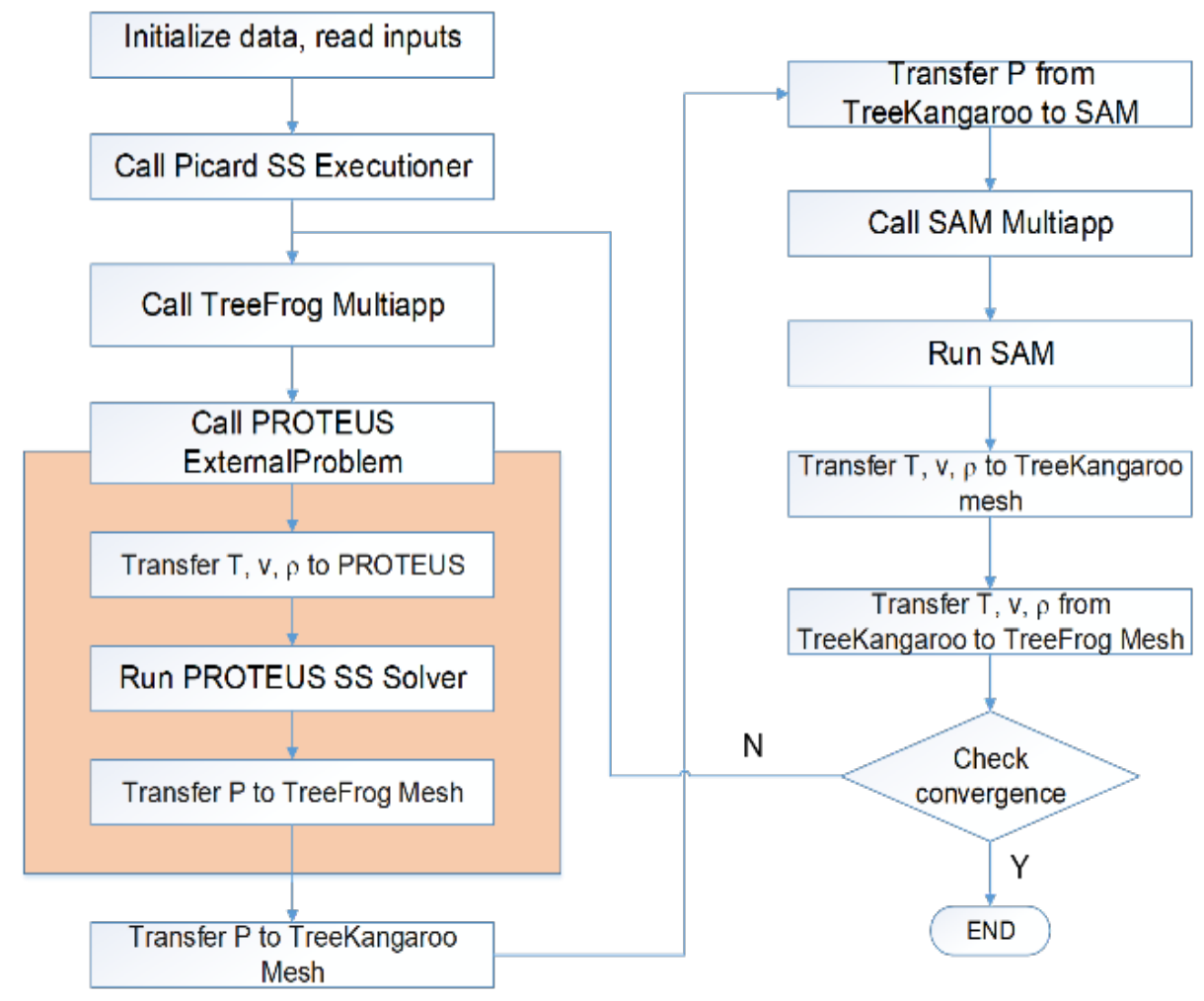

Fig. 4.4. Coupling scheme with Picard iteration for steady state calculations.

TreeFrog mesh and is transferred to SAM. Using the updated power, the SAM calculation is performed. At the end of each iteration, the SAM solution is transferred to PROTEUS-NODAL. The convergence is checked by comparing the maximum relative error in the power distribution between two successive Picard iterations. The Picard iteration is continued until the convergence criteria is met.

For transient calculations, the conventional operator splitting technique is used instead of the Picard iteration as shown in Fig. 4.5. Transient calculations are performed after SAM and PROTEUS-NODAL read their restart files that contain the steady state solutions as an initial condition. The restart file of SAM is the standard file defined in the MOOSE framework. The subroutines in PROTEUS-NODAL were modified to allow the time step size to be controlled by TreeFrog, not by the PROTEUS-NODAL input. As the time step size of the SAM calculation needs not be as small as that of the PROTEUS-NODAL calculation, the "subcycling" option defined in the transient executioner of the MOOSE framework is utilized to allow different time step sizes between PROTEUS-NODAL and SAM. With this option, sub-applications can take multiple time steps during a single time step of the master application. Thus, the time step size of TreeKangaroo is selected as the larger one of those 
specified in the PROTEUS-NODAL and SAM inputs. Within a one-time step of TreeKangaroo, the sub-application that has the larger time step size will be executed once and the other with smaller time step sizes will be executed multiple times until the time reaches the time of TreeKangaroo. Since the characteristic time in thermal-hydraulics calculations is usually larger than that in neutronics calculations, the time step size of TreeKangaroo is set to the same as that of SAM. If the SAM result does not converge, the SAM calculation is carried out again by halving the original time step size.

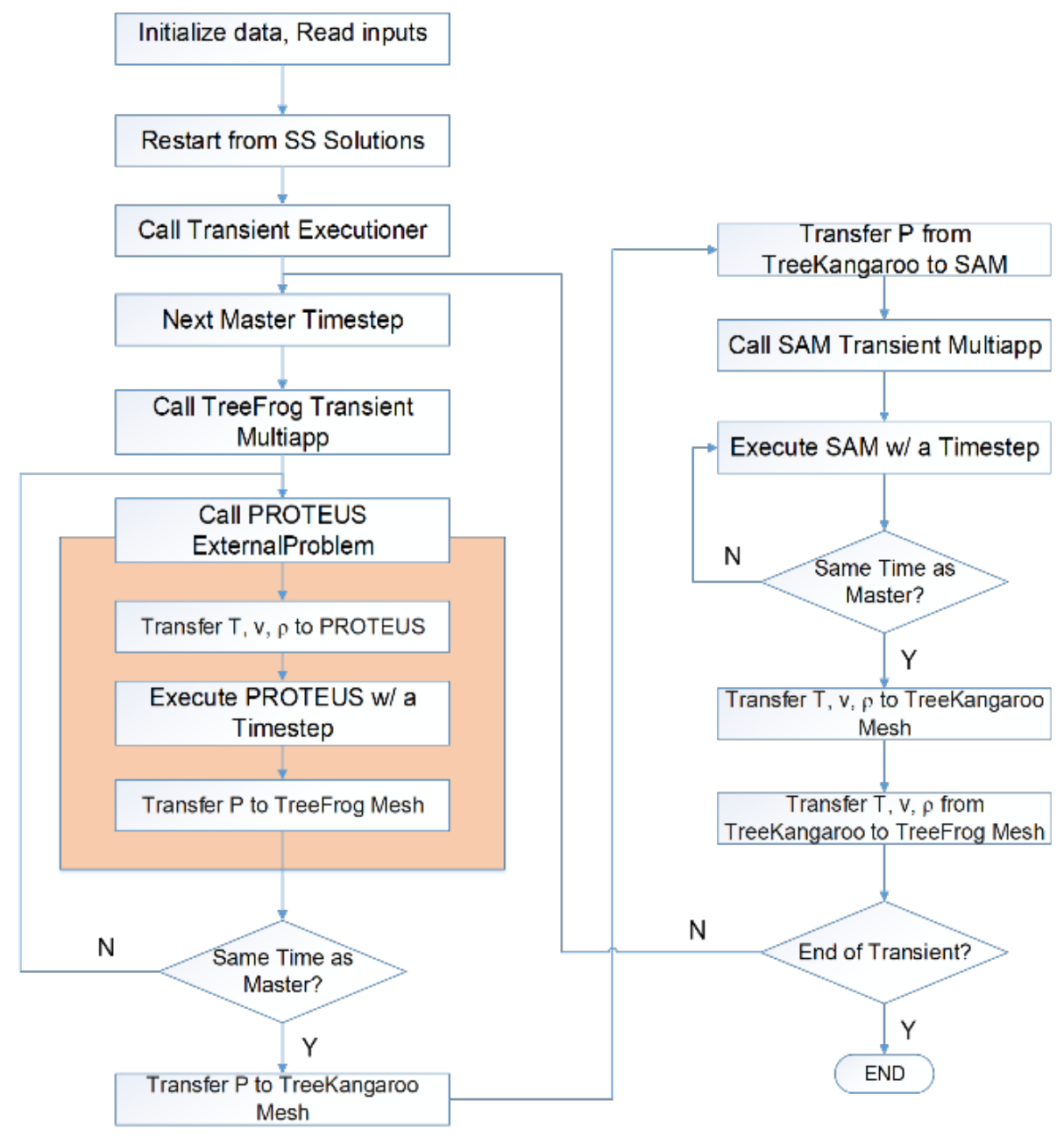

Fig. 4.5. Coupling scheme with operator splitting for transient calculations. 


\subsection{Verification Test Results}

Verification tests of the coupled system of PROTEUS-NODAL and SAM were performed using the steady state and transient problems derived from the MSFR benchmark problem [10]. Since the radial crossflow is neglected in the current SAM model coupled with PROTEUS-NODAL, the effect of this simplification was first examined by comparing the results of the coupled PROTEUS-NODAL and SAM calculation for a steady state problem with those obtained by a manually coupled calculation of PROTEUS-NODAL and the commercial CFD software ANSYS CFX [14]. In this comparison, the outer loop was not modeled, and the same inlet and outlet boundary conditions were used in both SAM and ANSYS CFX. Then, the MSFR transient benchmark problems were analyzed using the coupled system of PROTEUS-NODAL and SAM by including the outer loop in the SAM model. The results were compared with the other code system results found in the open literature.

\subsubsection{Comparison of Steady State Results of SAM with ANSYS CFX}

The coupled calculation results of PROTEUS-NODAL and SAM were compared with the manually coupled calculation results of PROTEUS-NODAL and CFX. For a consistent comparison, the outer loop was not included and only the core channels were used in the SAM model. The inlet velocity and temperature and the outlet pressure were given as the boundary conditions for both SAM and CFX calculations. The core was modeled with multiple parallel axial one-dimensional (1D) channels with the neglect of radial crossflow in the SAM calculation, while the full 3D Navier-Stokes equations were solved in the cylindrical domain in the CFX calculation. In both the SAM and CFX calculations, the thermo-physical properties of the fuel salt were taken from Reference [19] as provided in Appendix A for $\mathrm{LiF}$ (78 mol\%)-ThF 4 (22 mol\%) properties. For both coupled calculations, the PROTEUS-NODAL calculation was performed using the $\mathrm{P}_{1}$ solver in $\mathrm{R}-\mathrm{Z}$ geometry. The active core was divided into 20 axial regions and 6 radial rings. The first and second radial rings correspond to the first and second channels in the SAM model, respectively, the third and fourth rings correspond to the third channel, and the remaining fifth and sixth rings correspond to the fourth channel. Region-dependent cross-sections were generated using the $\mathrm{MC}^{2}-3$ code [20] with the ENDF/B-VII.0 data.

In both coupled calculations, the power densities of two successive Picard iterations were used to determine the convergence with a relative tolerance of $10^{-4}$. About three iterations were needed for convergence for this problem. In the manually coupled calculation of PROTEUS-NODAL and CFX, the initial power distribution was obtained from the coupled calculation of PROTEUS-NODAL and SAM. The power distribution of PROTEUSNODAL was fitted to a polynomial function. The third and fourth orders were used in the 
fitting for the radial and axial power distributions, respectively. Using this power distribution, the CFD simulation was performed to determine the flow fields of the fuel salt. In the subsequent PROTEUS-NODAL calculation, the radial velocity field of the CFX calculation was ignored and only the axial velocity field was used. Despite the different thermal-hydraulics modeling, the multiplication factor for the coupled calculation of PROTEUS-NODAL and CFX and that of PROTEUS-NODAL and SAM agreed very well within $2 \mathrm{pcm}$ ( 0.98117 with CFX and 0.98115 with SAM).

Fig. 4.6 shows the converged axial and radial velocity fields obtained from the coupled calculation of PROTEUS-NODAL and CFX. The axial velocity increases along the axial direction because of heating, and the axial velocity at the core outlet is higher in the central region than in the periphery region because of the higher power density. The high power density in the central region increases the fuel salt temperature and thus reduces its density, which in turn increases the flow velocity. The radial crossflow from the periphery region to the central region is also observed although the magnitude is smaller than $2 \%$ of the axial flow. (Both the positive velocity in the left half and the negative velocity in the right half are the flow from the periphery toward the core center.) Fig. 4.7 compares the axial velocity profiles of SAM and CFX. The axial velocity fields of SAM and CFX are quite different due to the neglect of the radial velocity field in the SAM calculation. The axial channel velocity of SAM increases along the axial direction in all the four channels, whereas that of CFX increases in the first three central channels and decreases in the fourth periphery channel. It is noted that the axial channel velocity of SAM is smaller than that of CFX in the three central channels.

Fig. 4.8 shows the power distribution obtained from the coupled PROTEUS-NODAL and SAM calculation and its relative difference from that obtained from the coupled PROTEUSNODAL and CFX calculation. The smaller axial velocities of SAM at the upper part of the three central channels reduce the delayed neutrons loss outside of the core and thus yield a relatively top-skewed power distribution. The relative difference from $-0.2 \%$ to $0.2 \%$ in the power distribution results in the difference in the fuel temperature distribution as shown in Fig. 4.9. The fuel temperature of SAM is slightly higher at the upper part of the core than that of CFX. 

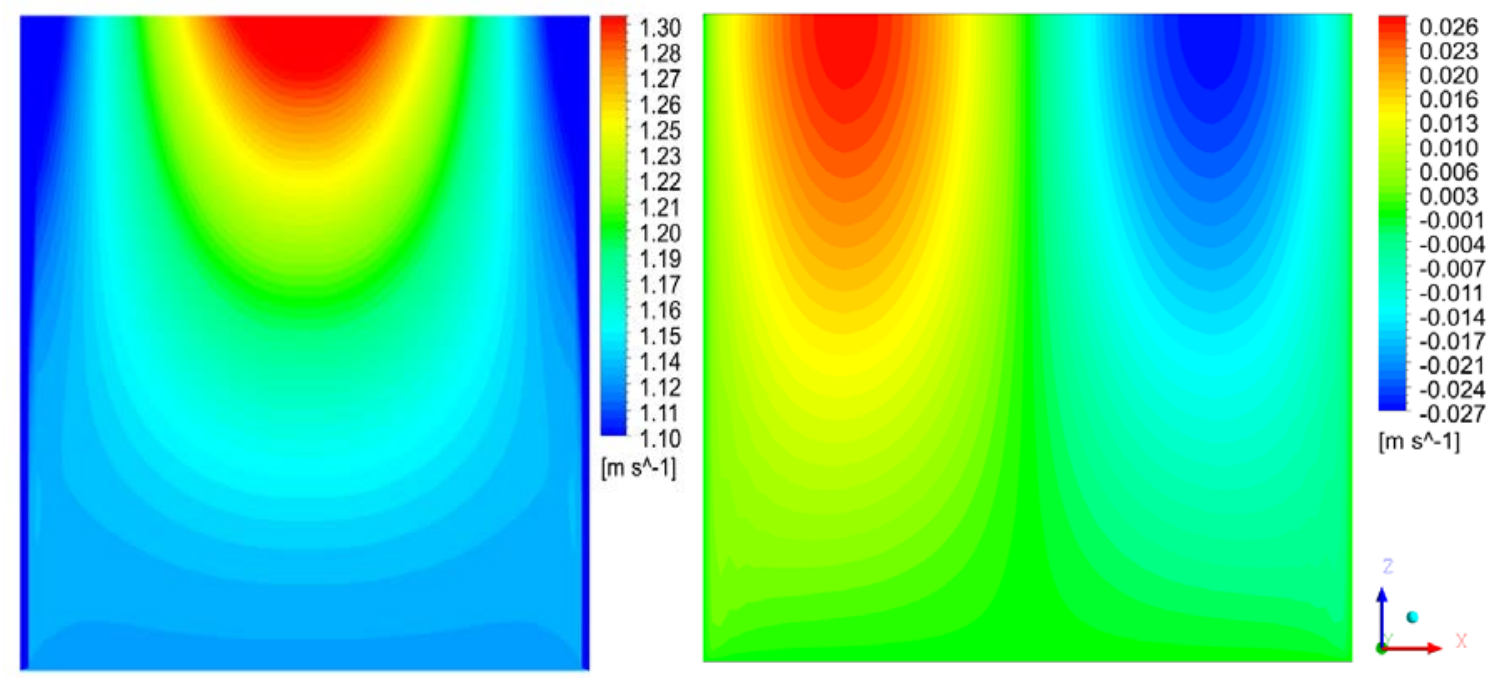

Fig. 4.6. Axial (left) and radial (right) velocity fields of the fuel salt obtained from CFX. The cylinder axis is in the middle of both figures.

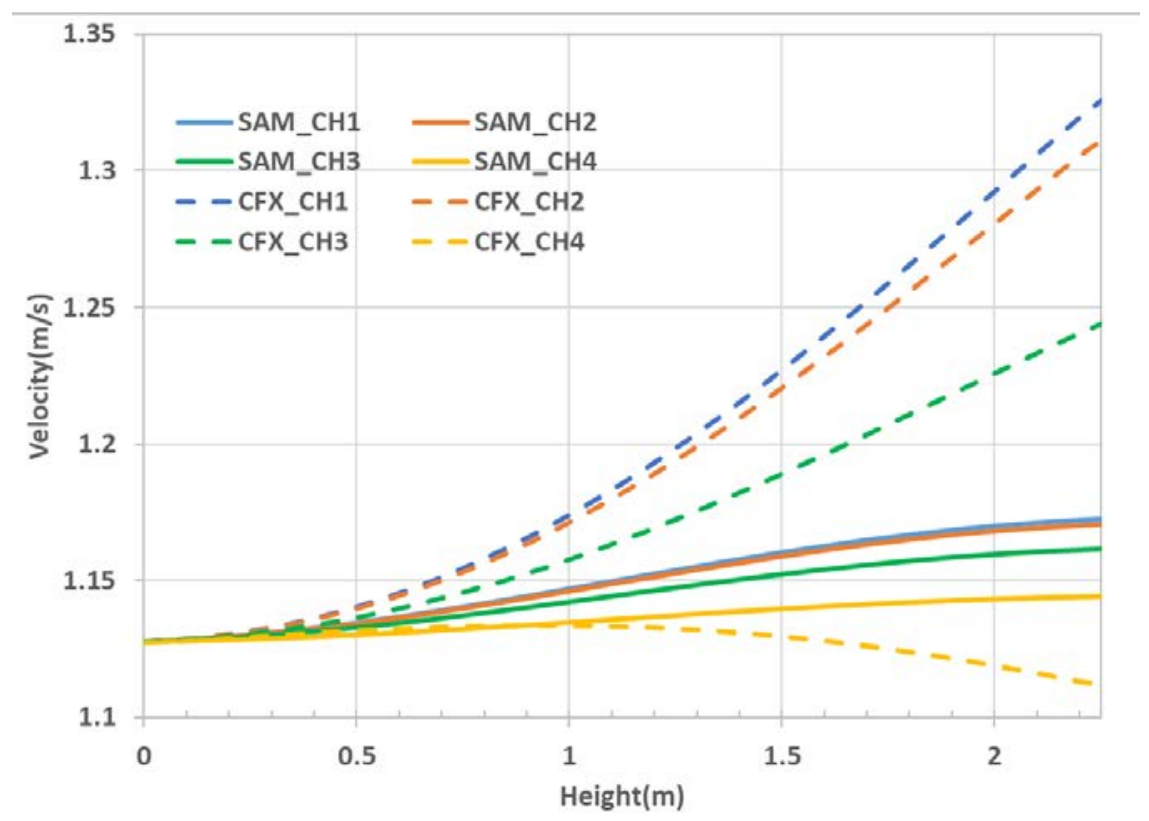

Fig. 4.7. Comparison of axial velocity profiles of SAM and CFX. 


\begin{tabular}{|l|l|l|l|}
\hline $2.61 \mathrm{E}+08$ & $2.50 \mathrm{E}+08$ & $1.98 \mathrm{E}+08$ & $9.72 \mathrm{E}+07$ \\
\hline $3.66 \mathrm{E}+08$ & $3.50 \mathrm{E}+08$ & $2.77 \mathrm{E}+08$ & $1.36 \mathrm{E}+08$ \\
\hline $4.68 \mathrm{E}+08$ & $4.48 \mathrm{E}+08$ & $3.54 \mathrm{E}+08$ & $1.75 \mathrm{E}+08$ \\
\hline $5.63 \mathrm{E}+08$ & $5.39 \mathrm{E}+08$ & $4.26 \mathrm{E}+08$ & $2.10 \mathrm{E}+08$ \\
\hline $6.43 \mathrm{E}+08$ & $6.17 \mathrm{E}+08$ & $4.88 \mathrm{E}+08$ & $2.41 \mathrm{E}+08$ \\
\hline $7.13 \mathrm{E}+08$ & $6.83 \mathrm{E}+08$ & $5.41 \mathrm{E}+08$ & $2.68 \mathrm{E}+08$ \\
\hline $7.64 \mathrm{E}+08$ & $7.32 \mathrm{E}+08$ & $5.81 \mathrm{E}+08$ & $2.88 \mathrm{E}+08$ \\
\hline $8.03 \mathrm{E}+08$ & $7.70 \mathrm{E}+08$ & $6.12 \mathrm{E}+08$ & $3.04 \mathrm{E}+08$ \\
\hline $8.23 \mathrm{E}+08$ & $7.90 \mathrm{E}+08$ & $6.28 \mathrm{E}+08$ & $3.13 \mathrm{E}+08$ \\
\hline $8.31 \mathrm{E}+08$ & $7.98 \mathrm{E}+08$ & $6.35 \mathrm{E}+08$ & $3.17 \mathrm{E}+08$ \\
\hline $8.20 \mathrm{E}+08$ & $7.87 \mathrm{E}+08$ & $6.27 \mathrm{E}+08$ & $3.14 \mathrm{E}+08$ \\
\hline $7.99 \mathrm{E}+08$ & $7.67 \mathrm{E}+08$ & $6.11 \mathrm{E}+08$ & $3.07 \mathrm{E}+08$ \\
\hline $7.59 \mathrm{E}+08$ & $7.29 \mathrm{E}+08$ & $5.83 \mathrm{E}+08$ & $2.93 \mathrm{E}+08$ \\
\hline $7.11 \mathrm{E}+08$ & $6.83 \mathrm{E}+08$ & $5.46 \mathrm{E}+08$ & $2.75 \mathrm{E}+08$ \\
\hline $6.49 \mathrm{E}+08$ & $6.23 \mathrm{E}+08$ & $4.99 \mathrm{E}+08$ & $2.52 \mathrm{E}+08$ \\
\hline $5.79 \mathrm{E}+08$ & $5.56 \mathrm{E}+08$ & $4.45 \mathrm{E}+08$ & $2.25 \mathrm{E}+08$ \\
\hline $4.98 \mathrm{E}+08$ & $4.79 \mathrm{E}+08$ & $3.84 \mathrm{E}+08$ & $1.94 \mathrm{E}+08$ \\
\hline $4.11 \mathrm{E}+08$ & $3.95 \mathrm{E}+08$ & $3.17 \mathrm{E}+08$ & $1.61 \mathrm{E}+08$ \\
\hline $3.19 \mathrm{E}+08$ & $3.07 \mathrm{E}+08$ & $2.46 \mathrm{E}+08$ & $1.25 \mathrm{E}+08$ \\
\hline $2.29 \mathrm{E}+08$ & $2.20 \mathrm{E}+08$ & $1.76 \mathrm{E}+08$ & $8.90 \mathrm{E}+07$ \\
\hline
\end{tabular}

(a)

\begin{tabular}{|c|c|c|c|}
\hline $1.14 \mathrm{E}-01$ & $1.19 \mathrm{E}-01$ & $1.28 \mathrm{E}-01$ & $1.53 \mathrm{E}-01$ \\
\hline $1.08 \mathrm{E}-01$ & $1.12 \mathrm{E}-01$ & $1.21 \mathrm{E}-01$ & $1.45 \mathrm{E}-01$ \\
\hline $1.01 \mathrm{E}-01$ & $1.07 \mathrm{E}-01$ & $1.12 \mathrm{E}-01$ & $1.34 \mathrm{E}-01$ \\
\hline $9.15 \mathrm{E}-02$ & $9.79 \mathrm{E}-02$ & $1.02 \mathrm{E}-01$ & $1.23 \mathrm{E}-01$ \\
\hline $8.10 \mathrm{E}-02$ & $8.68 \mathrm{E}-02$ & $8.93 \mathrm{E}-02$ & $1.08 \mathrm{E}-01$ \\
\hline $6.86 \mathrm{E}-02$ & $7.44 \mathrm{E}-02$ & $7.69 \mathrm{E}-02$ & $9.47 \mathrm{E}-02$ \\
\hline $4.97 \mathrm{E}-02$ & $5.40 \mathrm{E}-02$ & $5.96 \mathrm{E}-02$ & $7.92 \mathrm{E}-02$ \\
\hline $3.38 \mathrm{E}-02$ & $3.83 \mathrm{E}-02$ & $4.51 \mathrm{E}-02$ & $6.58 \mathrm{E}-02$ \\
\hline $5.93 \mathrm{E}-03$ & $8.96 \mathrm{E}-03$ & $2.30 \mathrm{E}-02$ & $5.12 \mathrm{E}-02$ \\
\hline$-1.29 \mathrm{E}-02$ & $-9.35 \mathrm{E}-03$ & $6.63 \mathrm{E}-03$ & $3.72 \mathrm{E}-02$ \\
\hline$-4.92 \mathrm{E}-02$ & $-4.64 \mathrm{E}-02$ & $-2.07 \mathrm{E}-02$ & $2.20 \mathrm{E}-02$ \\
\hline$-6.95 \mathrm{E}-02$ & $-6.61 \mathrm{E}-02$ & $-3.81 \mathrm{E}-02$ & $7.59 \mathrm{E}-03$ \\
\hline$-1.12 \mathrm{E}-01$ & $-1.09 \mathrm{E}-01$ & $-7.05 \mathrm{E}-02$ & $-1.01 \mathrm{E}-02$ \\
\hline$-1.31 \mathrm{E}-01$ & $-1.28 \mathrm{E}-01$ & $-8.76 \mathrm{E}-02$ & $-2.41 \mathrm{E}-02$ \\
\hline$-1.75 \mathrm{E}-01$ & $-1.72 \mathrm{E}-01$ & $-1.24 \mathrm{E}-01$ & $-4.49 \mathrm{E}-02$ \\
\hline$-1.90 \mathrm{E}-01$ & $-1.86 \mathrm{E}-01$ & $-1.38 \mathrm{E}-01$ & $-5.66 \mathrm{E}-02$ \\
\hline$-2.27 \mathrm{E}-01$ & $-2.23 \mathrm{E}-01$ & $-1.72 \mathrm{E}-01$ & $-7.74 \mathrm{E}-02$ \\
\hline$-2.29 \mathrm{E}-01$ & $-2.25 \mathrm{E}-01$ & $-1.76 \mathrm{E}-01$ & $-8.29 \mathrm{E}-02$ \\
\hline$-2.42 \mathrm{E}-01$ & $-2.39 \mathrm{E}-01$ & $-1.95 \mathrm{E}-01$ & $-9.33 \mathrm{E}-02$ \\
\hline$-2.09 \mathrm{E}-01$ & $-2.07 \mathrm{E}-01$ & $-1.70 \mathrm{E}-01$ & $-8.35 \mathrm{E}-02$ \\
\hline
\end{tabular}

(b)

Fig. 4.8. (a) Power distribution (W/cc) of coupled PROTEUS-NODAL and SAM calculation and (b) \% difference from that of coupled PROTEUS-NODAL and CFX calculation.

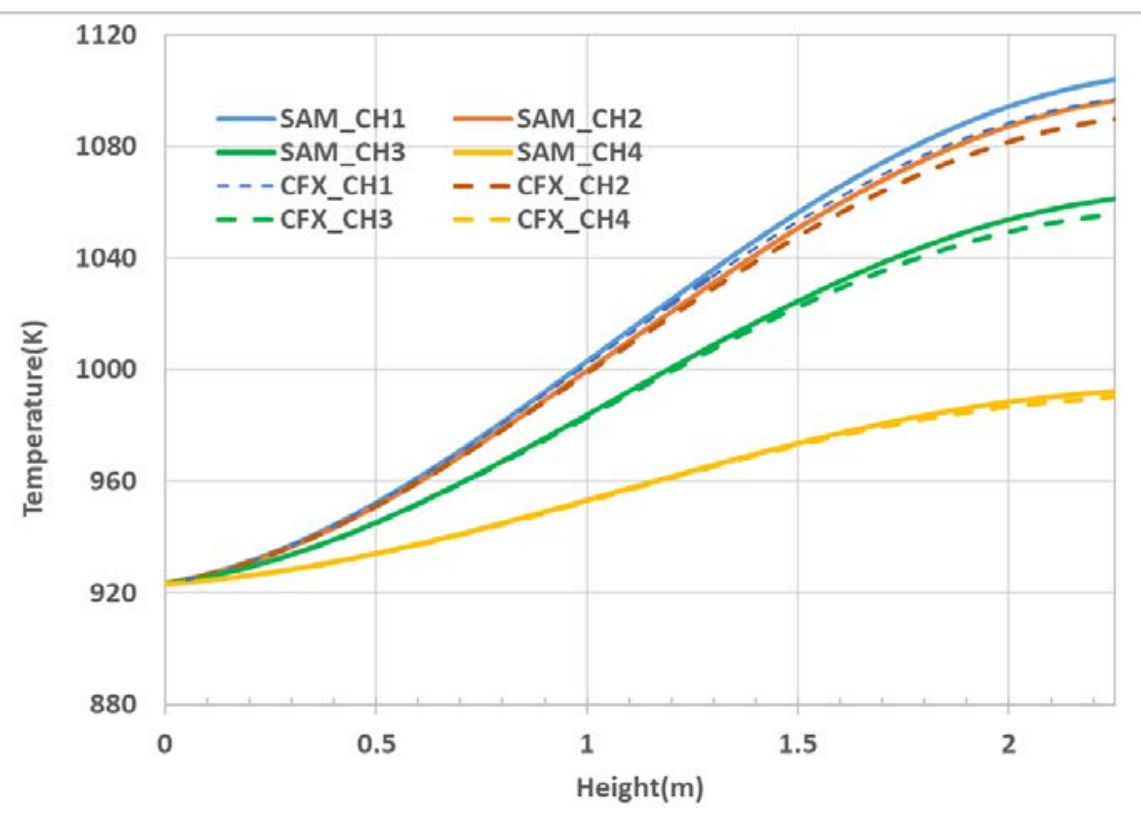

Fig. 4.9. Comparison of axial temperature profiles of SAM and CFX. 


\subsubsection{Transient Tests of Coupled System of PROTEUS-NODAL and SAM}

Three transient scenarios of the MSFR benchmark problem were analyzed using the coupled system of PROTEUS-NODAL and SAM: Unprotected Transient Over-Power (UTOP), Unprotected Loss of Flow (ULOF), and Unprotected Loss of Heat Sink (ULOHS) accidents. The PROTEUS-NODAL calculations were performed using the $\mathrm{P}_{1}$ solver in $\mathrm{R}-\mathrm{Z}$ geometry. The results were compared to the solutions in the open literature obtained with different code systems. One is the solution obtained from the coupled calculation with the nodal neutronics code PARCS and the system analysis code TRACE conducted at the Paul Scherrer Institute (PSI) [25]. The other is the solution obtained from the coupled calculation of a neutronics code based on the finite volume diffusion theory method and a CFD code conducted at the Delft University of Technology (TUDelft) [26].

Fig. 4.10 shows the SAM model for the MSFR benchmark problem. The core was divided into four axial 1D channels as in the steady state calculation. These channels were connected to zero-dimensional (0D) branch components. The heat exchanger and pump were connected to each other and the core channels were connected by pipes. Because of the lack of detailed design information, the heat exchanger and pump parameters were determined to yield the desired temperature increase across the core. The coolant inlet temperature at the secondary side of the heat exchanger was assumed $550^{\circ} \mathrm{C}$. The multigroup cross sections and thermo-physical properties of the fuel salt used in the steady state calculation were used for the transient analyses.

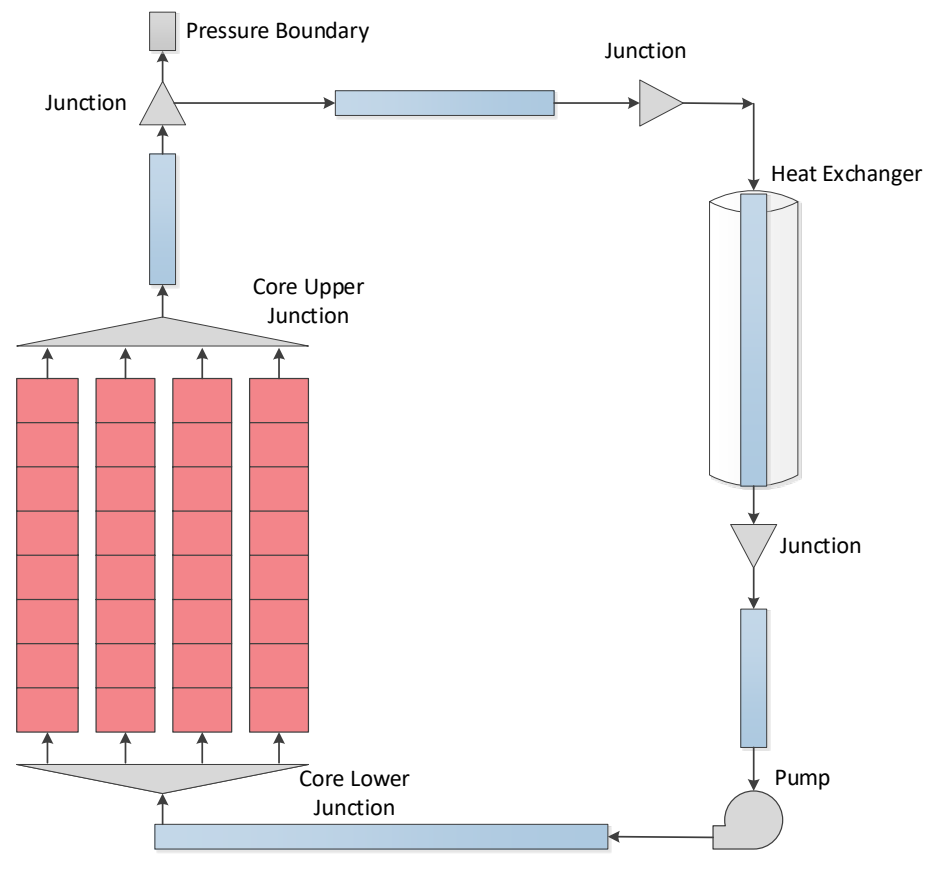

Fig. 4.10. SAM model of the MSFR benchmark problem. 
The UTOP transient was simulated by inserting a 50 pcm step reactivity at the beginning of the transient. This was achieved by increasing the fuel salt concentration in the core. Because of the rapid increase of the power at the beginning of the transient, the time step size for the PROTEUS-NODAL calculation was set to $0.0001 \mathrm{~s}$. On the other hand, since the response of thermal-hydraulics variables to the power change was relatively much slower, the larger time step size of $0.01 \mathrm{~s}$ was used in the SAM calculation. Fig. 4.11 compares the three power solutions from the coupled PROTEUS-NODAL and SAM calculation, the standalone PROTEUS-NODAL calculation with its thermal-hydraulics module, and Reference [26]. The power increases at the beginning of the transient and reaches its maximum ( 1.6 times of the nominal power) at around $4.9 \mathrm{~ms}$. The increased power increases the fuel salt temperature, which in turn introduces a negative reactivity due to the Doppler effects and the reduced fuel salt density. As a result, the power decreases after 4.9 ms. It can be seen that the power evolution of the coupled PROTEUS-NODAL and SAM calculation reasonably agrees well with the TUDelft results up to around $1 \mathrm{~s}$. However, the peak power predicted by PROTEUS-NODAL is slightly lower than the TUDelft result. This difference is attributed to the more negative temperature feedback coefficient of PROTEUSNODAL $(-7.68 \mathrm{pcm} / \mathrm{K})$ than that of TUDelft $(-6.79 \mathrm{pcm} / \mathrm{K})$ [9] due to the use of different cross section sets.

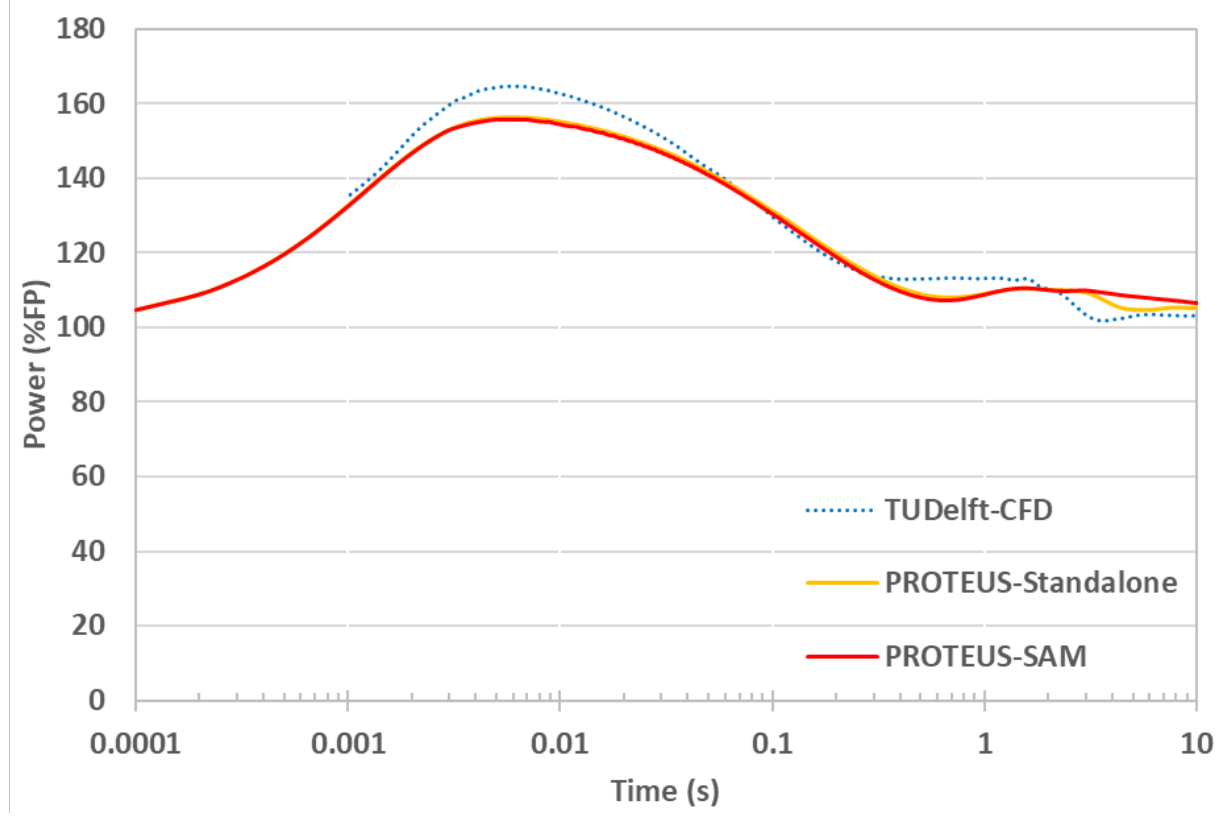

Fig. 4.11. Power evolution during the UTOP transient.

Fig. 4.12 shows the increase in the core-averaged temperature from the steady state as a function of time. The result of the coupled PROTEUS-NODAL and SAM calculation shows an increasing temperature after $\sim 2 \mathrm{~s}$ since the heated fuel salt comes back to the core inlet. 
On the other hand, the TUDelft result shows a decreasing temperature after $\sim 3 \mathrm{~s}$, which seems to be due to the decreasing power around $2 \mathrm{~s}$. The core-averaged fuel temperature predicted by PROTEUS-NODAL is about 3K lower than the TUDelft result at the end of 10 $\mathrm{s}$ transient because of the lower peak power caused by the more negative temperature coefficient. For this UTOP transient, the PROTEUS-NODAL standalone solution agrees very well with the result of the coupled PROTEUS-NODAL and SAM calculation until $2 \mathrm{~s}$. After $2 \mathrm{~s}$, the standalone PROTEUS-NODAL solution shows some deviation from the coupled calculation result because of the simplified outer loop model.

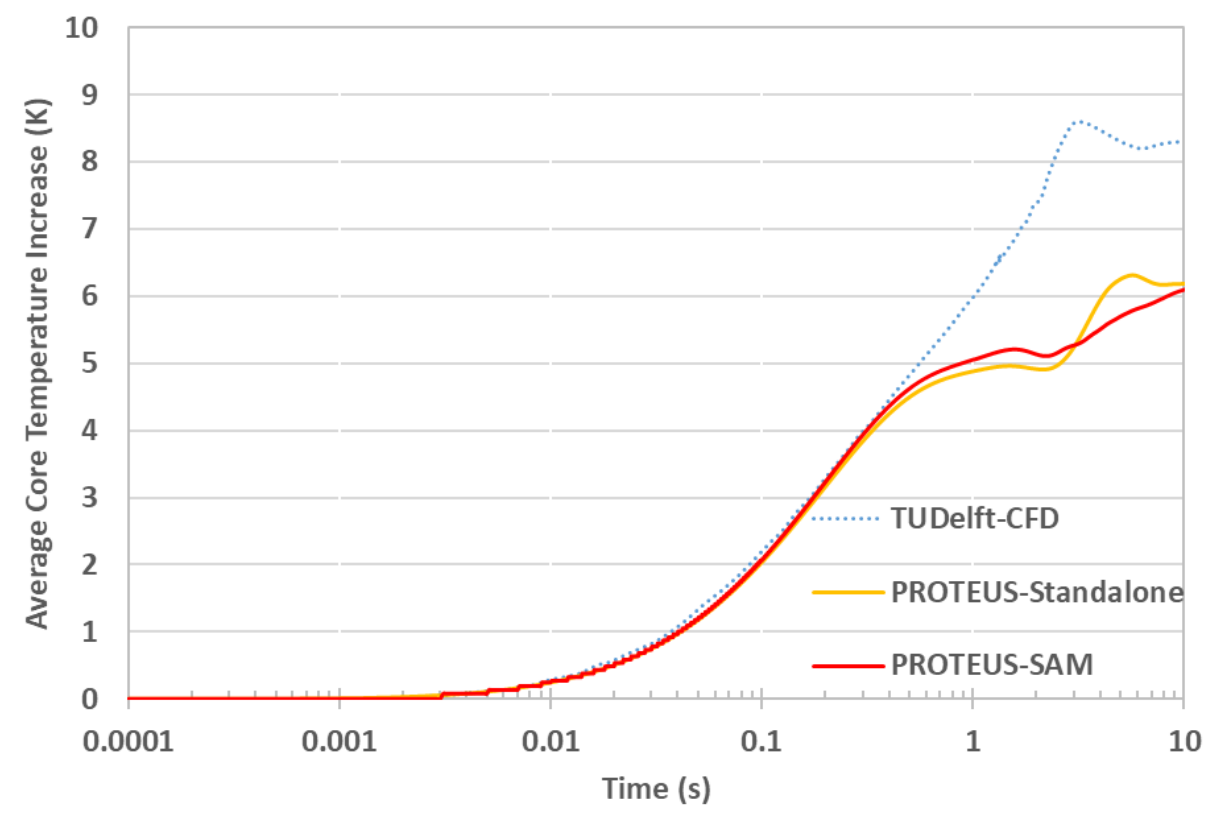

Fig. 4.12. Average core temperature increase during UTOP transient.

The ULOF transient is initiated by the pump failure. Since no secondary pump was included in the SAM model, the primary pump head and the secondary flow rate were decreased exponentially with a time constant of $5 \mathrm{~s}$. The decreased flow velocity reduces the number of delayed neutrons lost in the outer loop and thus introduces a positive reactivity. On the other hand, the reduced flow rate increases the core average temperature and thus induces a negative feedback due to the Doppler effects and the reduced fuel salt density. The negative feedback was larger than the positive feedback for this problem and thus the power decreases with time.

Fig. 4.13 compares the four power solutions from the coupled PROTEUS-NODAL and SAM calculation, the standalone PROTEUS-NODAL calculation with its thermal-hydraulics module, the coupled PARCS and TRACE calculation [25], and Reference [26]. The result of the coupled PROTEUS-NODAL and SAM calculation agrees well with that of TUDelft in 
the first $30 \mathrm{~s}$. The power decreases monotonically and reaches about $20 \%$ of the nominal full power at $30 \mathrm{~s}$. After $30 \mathrm{~s}$, the power obtained from the coupled PROTEUS-NODAL and SAM calculation keeps decreasing while the TUDelft power remains at about $20 \%$ of the nominal full power. This is because natural circulation was considered in the TUDelft model while not in the coupled PROTEUS-NODAL and SAM calculation.

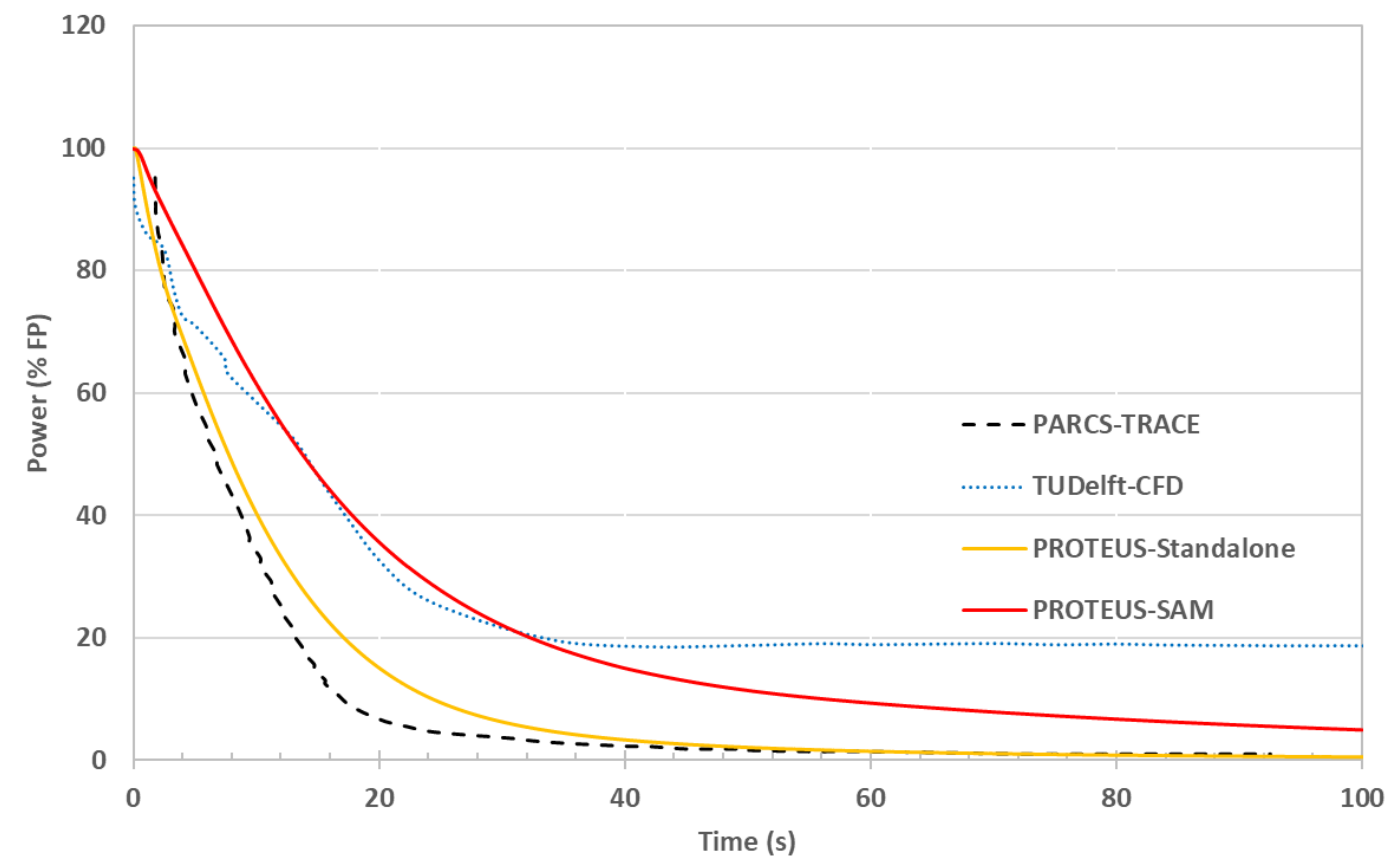

Fig. 4.13. Power evolution during ULOF transient.

The TUDelft power and that from coupled PROTEUS-NODAL and SAM calculation decrease more slowly than the other two solutions. This is due to the different outer loop models. While the inlet fuel salt velocity was directly decreased exponentially with a time constant of $5 \mathrm{~s}$ in the standalone PROTEUS-NODAL calculation with the thermal-hydraulics module and the coupled PARCS and TRACE calculation, the pump head was decreased in the calculation at TUDelft and in the coupled PROTEUS-NODAL and SAM calculation. As showed in Fig. 4.14, the average inlet velocity of the standalone PROTEUS-NODAL calculation with its thermal-hydraulics module decreases more rapidly than that of the coupled PROTEUS-NODAL and SAM calculation. The more rapid decrease in the inlet velocity leads to the more rapid decrease in the power. 


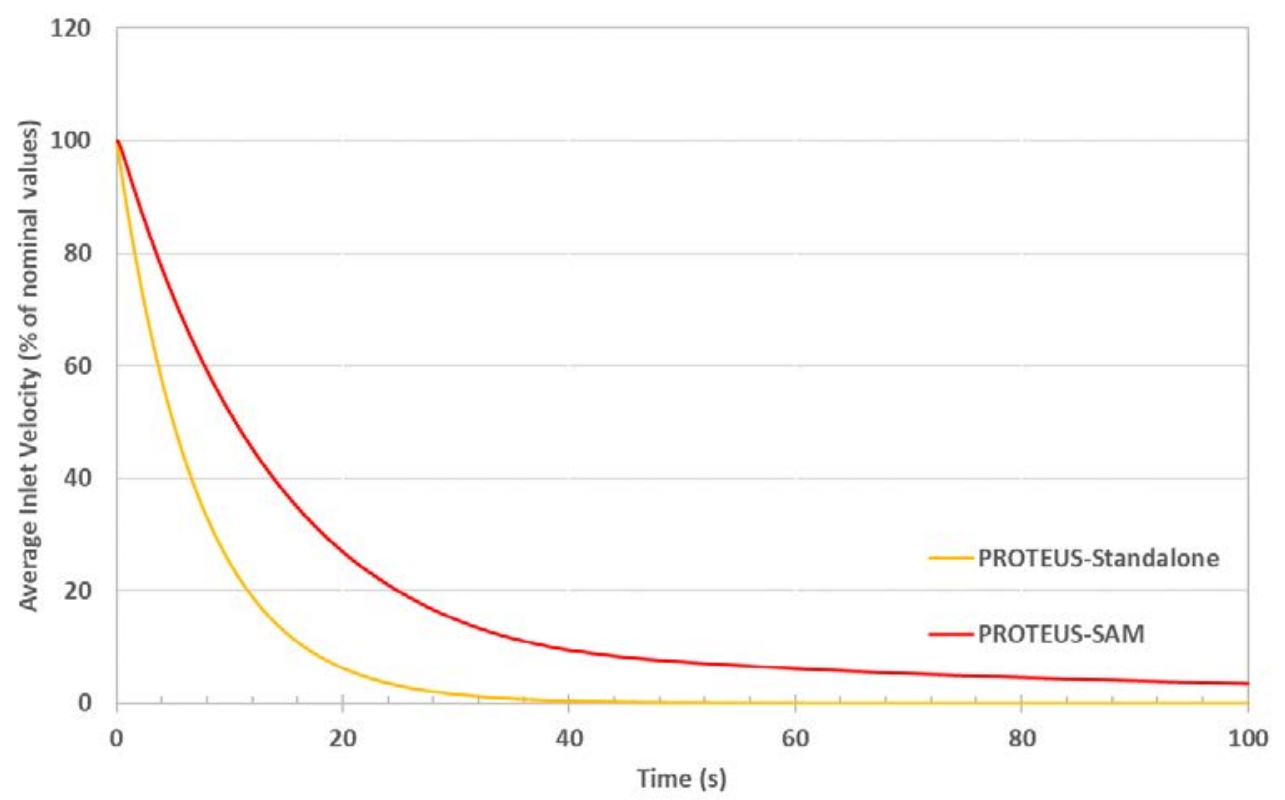

Fig. 4.14. Average inlet velocity evolution during ULOF transient.

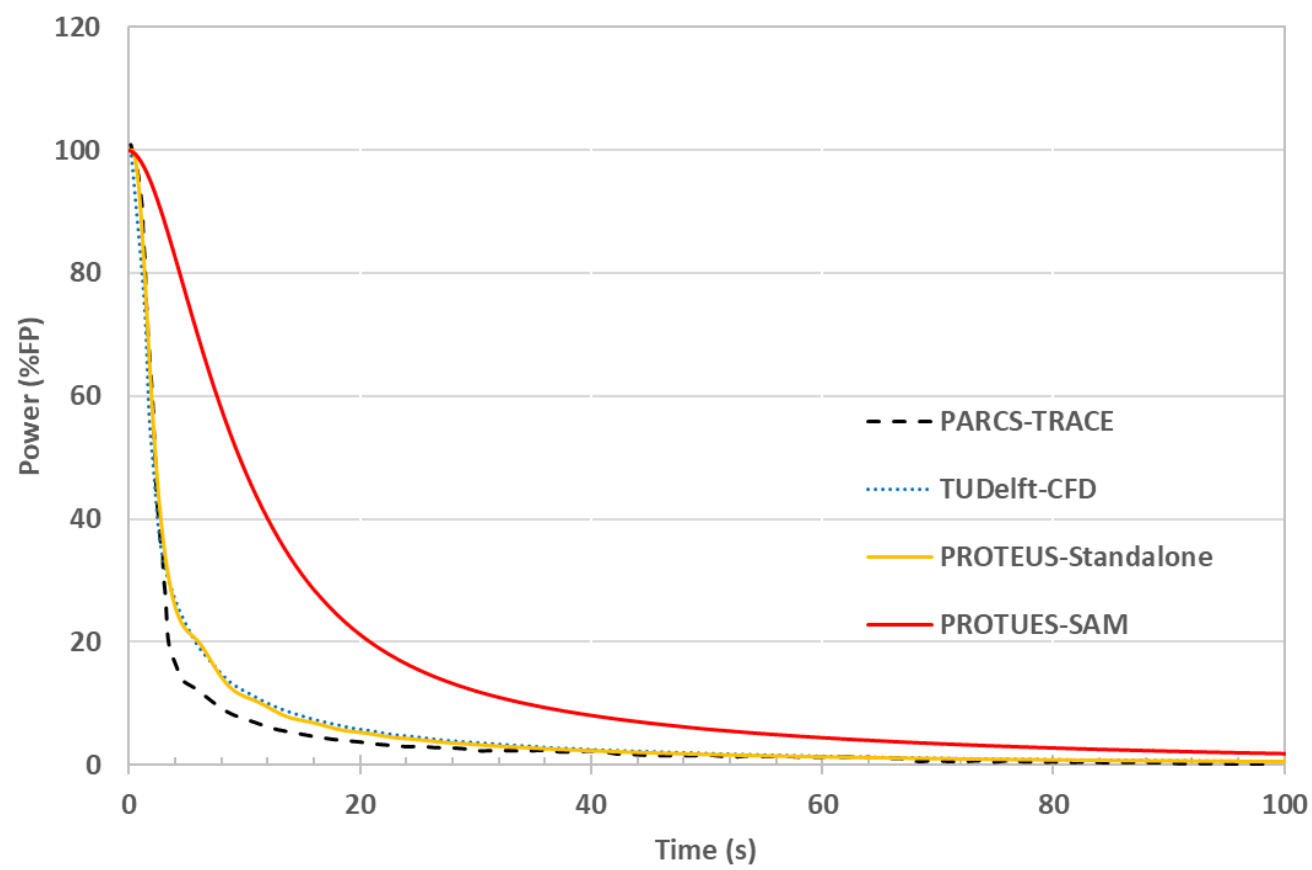

Fig. 4.15. Power evolution during ULOHS transient. 
The ULOHS transient was simulated by the exponential decrease in the inlet flow rate of the secondary side of the heat exchanger with a time constant of $1 \mathrm{~s}$ in the SAM calculation. For the standalone PROTEUS-NODAL calculation, the heat removal rate of the heat exchanger was manually decreased exponentially with a time constant of 1 s. Fig. 4.15 shows the power evolution during the ULOHS from the time when the uncooled fuel salt flows into the core inlet. Whereas the standalone PROTEUS-NODAL calculation result agrees well with the PARCS-TRACE and TUDelft results, the power obtained from the coupled PROTEUS-NODAL and SAM calculation decreases at a slower rate than others. This is due to the difference in the heat removal rate of the heat exchanger. 


\section{Summary}

In order to enhance the accuracy and efficiency of the PROTEUS-NODAL code for MSR simulations, several new capabilities have been implemented in the variational nodal $\mathrm{P}_{1}$ solver and the PROTEUS-NODAL code has been coupled with the system analysis module SAM. First of all, to eliminate the error caused by approximating a cylindrical MSR core by a hexagonal core, a variational nodal $\mathrm{P}_{1}$ method was developed for cylindrical geometries, i.e., R- $\theta-Z$, R- $\theta$, R-Z and $\mathrm{R}$ geometries. The developed capability was verified against the FDM solver of the DIF3D code using 2D and 3D steady state problems derived from the MSFR benchmark. The eigenvalue and power distribution results of the $\mathrm{P}_{1}$ solver in cylindrical geometries agreed very well with the reference solutions obtained by Richardson extrapolation of two DIF3D-FDM solutions. Relative to the fine hexagonal geometry model that yields a comparable accuracy, the computational time was reduced about 100 times by an R-Z model and about 7 to 8 times by an R- $\theta-Z$ model. Although the computational gain depends on the specific problem, it is obvious that a cylindrical geometry model is more efficient and accurate than a hexagonal geometry model for cylindrical geometry problems.

The transient analysis capability limited to the $\mathrm{SP}_{3}$ solver in hexagonal geometry was then extended to the $\mathrm{P}_{1}$ solvers for Cartesian, triangular, hexagonal, and cylindrical geometries. The TFSP solver was extended to the $\mathrm{P}_{1}$ solvers, and a FDM solver for the delayed neutron precursor equation was developed to overcome the limitations of the previously developed MOC and MoL solvers and to facilitate the coupling with the multi-dimensional flow models of SAM in the near future. To improve the computational efficiency for transient analyses, a CMFD acceleration scheme was also implemented. A capability to calculate the kinetics parameters in flowing fuel reactors was also added by implementing a steady state adjoint equation solver. The developed $\mathrm{P}_{1}$ transient solver was verified against the $\mathrm{SP}_{3}$ transient solver verified in the last year work using various transient problems in hexagonal geometry. The test results showed that the $\mathrm{P}_{1}$ solutions agree very well with the $\mathrm{SP}_{3}$ solutions. These tests also showed that the CMFD acceleration scheme reduces the computational time for the TFSP a few tens of times.

The $\mathrm{P}_{1}$ transient solver in $\mathrm{R}-\mathrm{Z}$ geometry was also tested by comparing the results with those of the hexagonal geometry option of the $\mathrm{P}_{1}$ solver and those of the $\mathrm{SP}_{3}$ solver. Various unprotected transient scenarios of the MSFR benchmark problem were solved with thermal feedback, including UTOP, UPOS, ULOF, ULOHS, and UFSOC accidents. Despite the differences in geometrical models and transport approximations (diffusion vs. $\mathrm{SP}_{3}$ ), the three solutions showed very good agreement in the power and core-averaged fuel salt temperature. The maximum difference in power was less than $0.9 \%$ for all the transients except for a super-prompt critical UTOP of a reactivity insertion of $200 \mathrm{pcm}$. In the latter case, the maximum difference was $2.0 \%$ for the $\mathrm{P}_{1}$ solver with $\mathrm{R}-\mathrm{Z}$ geometry and $5.0 \%$ for the $\mathrm{P}_{1}$ solver with hexagonal geometry. The maximum difference in the core-averaged fuel salt 
temperature was less than $0.8 \%$ for all the five scenarios. With these comparable accuracies, the $\mathrm{P}_{1}$ solver in R-Z geometry reduces the computational time about 10 to 60 times relative to the $\mathrm{SP}_{3}$ solver and about 5 to 10 times relative to the $\mathrm{P}_{1}$ solver in hexagonal geometry.

In order to enhance the thermal-hydraulics modeling capabilities by overcoming the limitation of the standalone thermal-hydraulics solver, PROTEUS-NODAL has been coupled with SAM under the MOOSE framework. A MOOSE sub-application named TreeFrog was developed as the wrapper for PROTEUS-NODAL to communicate with other MOOSE applications. A MOOSE master application named TreeKangaroo was also developed to control the coupling calculation of TreeFrog and SAM. Different time step sizes between PROTEUS-NODAL and SAM are allowed in the coupled transient calculations by using the "subcycling" option of the MOOSE transient executioner. The Picard iteration was used in the coupled steady state calculation, and the operator-splitting method was used in the coupled transient calculations.

Verification tests of the coupled system of PROTEUS-NODAL and SAM were performed using the steady state and transient problems derived from the MSFR benchmark problem. Since the radial crossflow is neglected in the current SAM model, the effect of this simplification was first examined by comparing the steady state results with those obtained by a manually coupled calculation of PROTEUS-NODAL and ANSYS CFX. In this comparison, the outer loop was not modeled, and the same inlet and outlet boundary conditions were used in both SAM and ANSYS CFX. The SAM calculation used four parallel axial channels and CFX performed the full 3D CFD calculation in the cylindrical geometry of the MSFR core. Due to the neglect of the radial velocity field in the SAM calculation, SAM underestimated the axial velocity at the core center slightly, and this resulted in a slightly top-skewed power distribution: $0.1 \%$ overestimation in the upper part and $0.2 \%$ underestimation in the lower part of the core. The UTOP, ULOF, and ULOHS accidents of the MSFR transient benchmark were analyzed by including the outer loop in the SAM model. The results were compared with the PSI solutions from a coupled PARCS and TRACE calculation and the TUDelft solutions obtained from a coupled neutron diffusion and CFD calculation. In general, the power and core-averaged fuel temperature solutions of the coupled PROTEUS-NODAL and SAM calculations agreed well with the other solutions.

Future work will focus on the coupling of PROTEUS-NODAL with the recently developed 3D SAM to consider the radial crossflow of the fuel salt and the implementation of the transport kernel of the variational nodal method in the $\mathrm{P}_{1}$ solver to model the transport effects more accurately. In addition, validation tests will be performed using the experimental data of the molten salt reactor experiment (MSRE). 


\section{References}

1. L. Pioro, Handbook of Generation IV Nuclear Reactors: Introduction Generation IV International Forum, Woodhead Publishing (2016).

2. M. Allibert, et al., Handbook of Generation IV Nuclear Reactors: Molten Salt Fast Reactors, Woodhead Publishing (2016).

3. E. Merle-Lucotte, et al., "Launching the Thorium Fuel Cycle with the Molten Salt Fast Reactor,” Proc. of ICAPP 2011, Nice, France, May 2-5 (2011)

4. C. H. Lee, Y. S. Jung, and M. A. Smith, "FY17 Status Report on NEAMS Neutronics Activities,” ANL/NE-17/28, Argonne National Laboratory (2017).

5. Y. S. Jung, M. A. Smith, and C. H. Lee, "PROTEUS-NODAL User Manual," ANL/NE18/4, Argonne National Laboratory (2018).

6. G. Palmiotti, et al., "VARIANT: VARIational Anisotropic Nodal Transport for Multidimensional Cartesian and Hexagonal Geometry Calculation,” ANL-95/40, Argonne National Laboratory (1995).

7. M. Bae, "Development of Triangle-based Polynomial Expansion Nodal $\mathrm{SP}_{3}$ Method for Hexagonal Core Transport Calculation,” M.S. Thesis, Seoul National University (2010).

8. Y. S. Jung, et al., "Molten Salt Reactor Analysis Capability of PROTEUS-NODAL," Trans. Am. Nucl. Soc, 119, 1131-1134 (2018).

9. M. K. Jaradat and W. S. Yang, "Development of Molten Salt Reactor Analysis Capabilities of the NEAMS Neutronics Code PROTEUS-NODAL,” UM/NRDSL-19/01, University of Michigan (2019).

10. M. K. Jaradat, et al., "Molten Salt Reactor Transient Analysis Capability of PROTEUSNODAL,” Proc. of Global 2019, Seattle, USA, September 22-26 (2019).

11. R. Hu, L. Zou, and G. Hu, “SAM User’s Guide,” ANL/NSE-19/18, Argonne National Laboratory (2019).

12. D. Gaston, et. al., "Physics-based Multiscale Coupling for Full Core Nuclear Reactor Simulation,” Ann. Nucl. Energy, 84, 45-54 (2015).

13. K. L. Derstine, "DIF3D: A Code to Solve One-, Two-, and Three-Dimensional FiniteDifference Diffusion Theory Problems,” ANL-82-64, Argonne National Laboratory (1984).

14. ANSYS ${ }^{\circledR}$ CFX, Release 18.0, ANYSYS, Inc.

15. M. A. Smith, E. E. Lewis, and E. R. Shemon, "DIF3D-VARIANT 11.0: A Decade of Updates,” ANL/NE-14/1, Argonne National Laboratory (2014).

16. C. H. Lee and Y. S. Jung, "FY18 Progress on Improvements and V\&V of $M^{2}-3$ and PROTEUS,” ANL/NSE-18/9, Argonne National Laboratory (2018). 
17. S. Zhou, et al., "Fuel Cycle Analysis of Molten Salt Reactors Based on Coupled Neutronics and Thermal-hydraulics Calculations,” Annals of Nuclear Energy, 114, 369 (2018).

18. M. Aufiero, et al., "Calculating the effective delayed neutron fraction in the Molten Salt Fast Reactor: analytical, deterministic and Monte Carlo approaches,” Annals of Nuclear Energy, 65, 78, (2014).

19. Brovchenko, et al., "Optimization of the Pre-conceptual Design of the MSFR: Evaluation and Viability of Liquid Fuel Fast Reactor System,” EVOL Project Deliverable EVOL D2 (2) (2013).

20. C. Lee and W. Yang, " $M C^{2}-3$ Multigroup Cross Section Generation Code for Fast Reactor Analysis,” Nuclear Science and Engineering, 187, 268-290 (2017).

21. J. Leppanen, et al., "The Serpent Monte Carlo Code: Status, Development and Applications in 2013,” Annals of Nuclear Energy, 82, 142-150 (2015).

22. P. Romano, et al., “OpenMC: A State-of-the-art Monte Carlo Code for Research and Development,” Annals of Nuclear Energy, 82, 90-97 (2015).

23. C. H. Lee and Y. S. Jung, "Generation of the Cross Section Library for PROTEUS," ANL/NE-18/2, Argonne National Laboratory (2018).

24. A. E. Waltar, D. R. Todd, and P. V. Tsvetkov, Fast Spectrum Reactors, Springer (2012).

25. C. Fiorina, et al., "Modelling and Analysis of the MSFR Transient Behavior," Annals of Nuclear Energy, 64, 485 (2014).

26. E. Pettersen, “Coupled Multi-physics Simulations of the Molten Salt Fast Reactor Using Coarse-mesh Thermal-hydraulics and Spatial Neutronics,” M.S. Thesis, University ParisSaclay \& Paul Scherrer Institute (2016).

27. V. Ignatiev, et al., "Progress in Development of MOSART Concept with Th Support," Proc. of ICAPP 2012, Chicago, USA, June 24-28 (2012).

28. C. Guerrieri, et al., “A Preliminary Study of the MSFR Dynamics,” Proc. of $20^{\text {th }}$ International Conference on Nuclear Engineering, Anaheim, California, USA, July 30 August 3 (2012).

29. N. E. Todreas and M. S. Kazimi, Nuclear Systems Vol. I: Thermal Hydraulic Fundamentals, 2nd Ed., CRC Press (2012).

30. B. S. Petukhov, "Heat Transfer and Friction in Turbulent Pipe Flow with Variable Physical Properties,” Advances in Heat Transfer, 6, 503 (1970).

31. "RELAP5/MOD3.3 Code Manual,” Nuclear Safety Analysis Division, Information Systems Laboratories, Inc., Rockville, MD (2002).

32. W. S. Yang, T. Jing, Y. S. Jung, S. Shi, and G. Yang, "Stationary Liquid Fuel Fast Reactor,” PU/NE-15/08, Purdue University (2015). 
33. V. Gnielinski, "New Equations for Heat and Mass Transfer in Turbulent Pipe and Channel Flow,” International Chemical Engineering, 16, 359 (1976). 


\section{Appendix A. Description of MSFR Benchmark Problem}

The MSFR design specification and relevant material properties are mainly obtained from the EVOL (Evaluation and Viability of Liquid Fuel Fast Reactor) project report [19]. The MSFR design has a thermal power of $3000 \mathrm{MW}_{\text {th }}$ and utilizes a fast neutron spectrum based on the thorium fuel cycle. The MSFR utilizes a binary fluoride salt, composed of LiF (77.5 molar \%) and a heavy nuclei (HN) mixture (22.5 molar \%) initially composed of fertile thorium and fissile material. The fuel salt is circulated in the core and in the external loop, which consists of 16 branches each with the heat exchanger, pump, and associated instruments and pipes. A schematic representation of the MSFR core is shown in Fig. A.1.

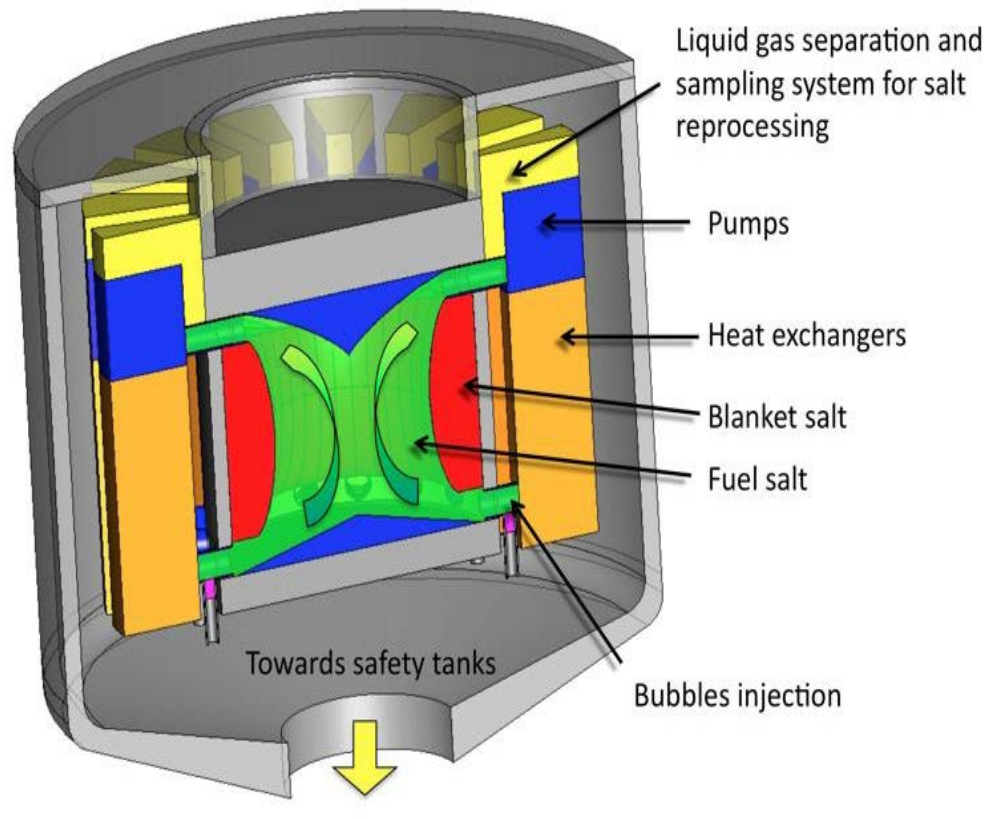

Fig. A.1. Schematic representation of the molten salt fast reactor.

The MSFR is operated between $650^{\circ} \mathrm{C}$ and $750{ }^{\circ} \mathrm{C}$ with a circulation time of $4.0 \mathrm{~s}$, which can be controlled based on the power level and the temperature rise in the core. The fuel salt temperature rise across the core during normal operation is fixed at $100{ }^{\circ} \mathrm{C}$. The active reactor core has a cylindrical shape with the same height and diameter of $2.255 \mathrm{~m}$ to improve the breeding ratio and reduce the neutron leakage. The total fuel salt volume is $18 \mathrm{~m}^{3}$, half of which is in the active core. The radial reflector includes a fertile blanket of $50 \mathrm{~cm}$ thick to increase the breeding ratio and to shield the external components, and it is filled with a fertile salt of $\mathrm{LiF}_{-T h F}$ (same type as the core salt). The walls of the blanket are surrounded by a 20 $\mathrm{cm}$ thick layer of $\mathrm{B}_{4} \mathrm{C}$ to enhance the shielding of the external components. The external core 
structures and the heat exchangers are protected by thick reflectors made of nickel-based alloys for corrosion resistance. Table A.1 provides the main characteristics of the MSFR core.

Table A.1. Characteristics of the Molten Salt Fast Reactor

\begin{tabular}{|l|l|}
\hline Thermal/electric power Fuel salt & $3000 \mathrm{MWth} / 1300 \mathrm{MWe}$ \\
\hline Temperature increase in the core & $100{ }^{\circ} \mathrm{C}$ \\
\hline Fuel salt melting point & $565^{\circ} \mathrm{C}$ \\
\hline Fuel Inlet/outlet temperature & $650^{\circ} \mathrm{C} / 750{ }^{\circ} \mathrm{C}$ \\
\hline Mean fuel salt temperature & $700{ }^{\circ} \mathrm{C}$ \\
\hline Fuel salt density & $4.1249 \mathrm{~g} / \mathrm{cm}^{3}$ \\
\hline Core Height / Core Radius & $2.255 \mathrm{~m} / 1.1275 \mathrm{~m}$ \\
\hline Fuel salt volume & $18 \mathrm{~m}^{3}$ \\
\hline Total fuel salt cycle in the fuel circuit & $4.0 \mathrm{~s}$ \\
\hline Flow rate & $4.5 \mathrm{~m}^{3} / \mathrm{s}$ \\
\hline
\end{tabular}

Fuel salt in its initial composition has a fissile element either ${ }^{233} U\left({ }^{233} U\right.$-started) or the transuranic elements produced by PWRs (TRU-started). The ${ }^{233} \mathrm{U}$-started fuel salt is composed of $\mathrm{LiF}_{-} \mathrm{ThF}_{4}{ }^{2}{ }^{23} \mathrm{UF}_{3}$, and the TRU-started fuel salt is composed of $\mathrm{LiF}_{-} \mathrm{ThF}_{4}$ (TRU)F $F_{3}$ with a TRU mixture of Pu (87.5\%), Np (6.3\%), Am (5.3\%), and Cm (0.9\%). The initial fuel compositions of both options are provided in Table A.2, and the isotopic compositions of the TRU element are listed in Table A.3.

Table A.2. Initial Composition of the Fuel Salt of MSFR

\begin{tabular}{|c|c|c|c|}
\hline \multicolumn{2}{|c|}{${ }^{23}$ U-started } & \multicolumn{2}{c|}{ TRU-started } \\
\hline Element & Mole fraction (\%) & Element & Mole fraction (\%) \\
\hline Th & 19.985 & Th & 16.068 \\
\hline U-233 & 2.515 & $\mathrm{Pu}$ & 5.628 \\
\hline & & $\mathrm{Np}$ & 0.405 \\
\hline & & $\mathrm{Am}$ & 0.341 \\
\hline & & $\mathrm{Cm}$ & 0.058 \\
\hline
\end{tabular}

Table A.3. TRU-element Fuel Composition

\begin{tabular}{|c|c|c|c|}
\hline Isotope & Mole fraction (\%) & Isotope & Mole fraction (\%) \\
\hline Pu-238 & 2.7 & Np-237 & 6.3 \\
\hline Pu-239 & 45.9 & Cm-244 & 0.8 \\
\hline Pu-240 & 21.5 & Cm-245 & 0.1 \\
\hline $\mathrm{Pu}-241$ & 10.7 & Am-241 & 3.4 \\
\hline $\mathrm{Pu}-242$ & 6.7 & Am-243 & 1.9 \\
\hline
\end{tabular}


The MSFR utilizes 16 heat exchangers and 16 pumps. Each heat exchanger has the capability of extracting $187 \mathrm{MW}$ during normal operation, and each pump provides a flow rate of about $0.28 \mathrm{~m}^{3} / \mathrm{s}$ to maintain the temperature rise in the core. The heat exchanger considered in the following simulations consists of about 8000 tubes with a length of $2.0 \mathrm{~m}$ and an inner diameter of $0.4 \mathrm{~cm}$, with a fuel salt volume of $0.203 \mathrm{~m}^{3}$. The inlet and outlet temperatures of the heat exchanger are $750{ }^{\circ} \mathrm{C}$ and $650{ }^{\circ} \mathrm{C}$, respectively. The MSFR design includes an intermediate circuit that is used to separate the radioactive fuel salt from the energy conversion system that uses FLiNaK as a coolant with a temperature of $550{ }^{\circ} \mathrm{C}$. The heat exchanger is assumed to be of shell and tube type as the MSRE and MSBR designs developed at the Oak Ridge National Laboratory. The temperature range of the MSFR design is between $838 \mathrm{~K}$ (the salt freezing point) and $1600 \mathrm{~K}$ (the melting point of the nickel alloy of the core structures).

Table A.4 provides the thermo-physical properties for salt of $\mathrm{LiF}$ (78 mol\%)- $\mathrm{ThF}_{4}$ (22 mol\%) as provided in reference [27]. It is assumed that the fission products and new heavy nuclei produced during reactor operation will not impact the fuel salt thermo-physical properties.

Table A.4. Thermo-physical Properties of LiF-ThF 4 Fuel Salt

\begin{tabular}{|l|l|c|}
\hline Property & Correlation & $\begin{array}{c}\text { Validity } \\
\text { Range }\left[{ }^{\circ} \mathrm{C}\right]\end{array}$ \\
\hline Density $\left[\mathrm{kg} / \mathrm{m}^{3}\right]$ & $\rho=4094-0.882(T[\mathrm{~K}]-1008)$ & $620-850$ \\
\hline Kinematic Viscosity $\left[\mathrm{m}^{2} / \mathrm{s}\right]$ & $v=5.54 \times 10^{-8} e^{3689 / T[\mathrm{~K}]}$ & $625-846$ \\
\hline Dynamic Viscosity [Pa.s] & $\mu=\left[2.268-4.886 \times 10^{-4}(T[\mathrm{~K}]-1008)\right] \times 10^{-4} e^{3689 / T[\mathrm{~K}]}$ & $625-846$ \\
\hline Thermal Conductivity [W/m.K] & $k=0.928+8.397 \times 10^{-5} T[\mathrm{~K}]$ & $618-747$ \\
\hline Specific Heat $[\mathrm{J} / \mathrm{kg} . \mathrm{K}]$ & $c_{p}=-1111+2.78 T[\mathrm{~K}]$ & $594-634$ \\
\hline
\end{tabular}

In order to perform decay heat calculations, the decay heat fractions, and decay constants should be known. The parameters in Reference [28] were adopted in this study. Table A.5 provides the decay heat fractions and decay constants for TRU and ${ }^{233} \mathrm{U}$ started MSFR.

Table A.5. Decay Heat Parameters of MSFR

\begin{tabular}{|c|c|c|c|c|}
\hline \multirow{2}{*}{ Group } & \multicolumn{2}{|c|}{${ }^{233}$ U-started } & \multicolumn{2}{c|}{ TRU-Started } \\
\cline { 2 - 5 } & Fraction (\%) & Decay Constant $\left(\mathrm{s}^{-1}\right)$ & Fraction (\%) & Decay Constant $\left(\mathrm{s}^{-1}\right)$ \\
\hline 1 & 1.28 & 0.2160 & 1.39 & 0.2340 \\
\hline 2 & 1.23 & 0.0182 & 1.21 & 0.0205 \\
\hline 3 & 2.08 & $2.590 \times 10^{-4}$ & 1.63 & $3.180 \times 10^{-4}$ \\
\hline Total & 4.59 & & 4.23 & \\
\hline
\end{tabular}




\section{Appendix B. Comparison of Three Solution Methods for Delayed Neutron Precursor Equation of Flowing Fuel}

As discussed in Section 3.2, three different numerical methods were examined to solve the delayed neutron precursor equation for 1D axial flow: the method of characteristics (MOC), the method of lines (MoL), and the finite difference method (FDM). FDM was selected and implemented in PROTEUS-NODAL since it produces the null transient result correctly and it can be easily extended to multi-dimensional flows. The FDM method was discussed in Section 3.2, and thus in this appendix, MOC and MoL are discussed and their results for three transient scenarios are compared with the FDM solutions.

\section{B.1. Solution Methods for 1D Axial Flow}

\section{B.1.1. Method of Characteristics}

Under the assumption that the fuel velocity is constant within a node, the precursor equation for 1D axial flow given in Eq. (3.11) can be solved analytically using the MOC. Along a characteristic line that can be represented by a parameter $s$, the terms including the temporal and spatial derivatives can be represented by the total derivative with respect to $s$. Denoting the precursor concentration of family $k$ in a radial mesh $i$ by $C_{k, i}$, its derivative with respect to $s$ becomes

$$
\frac{d}{d s} C_{k, i}[z(s), t(s)]=\frac{\partial C_{k, i}}{\partial t} \frac{d t}{d s}+\frac{\partial C_{k, i}}{\partial z} \frac{d z}{d s},
$$

where $i$ refers to the index for a radial mesh. Therefore, Eq. (3.11) can be written as an ordinary differential equation (ODE) as

$$
\frac{d}{d s} C_{k, i}(s)+\lambda_{k} C_{k, i}(s)=\lambda \psi_{k, i}(s),
$$

with the following auxiliary ODEs for the characteristic line:

$$
\frac{d t}{d s}=1, \frac{d z}{d s}=u_{i} .
$$

The characteristic lines can be determined by solving Eq. (B.3) as

$$
\left\{\begin{array}{l}
t=s+t_{0} \\
z=u_{i} s+z_{0}
\end{array} .\right.
$$

where $t_{0}$ and $z_{0}$ are the coordinates for the starting point where the initial or boundary condition is given. Along a characteristic line, the ODE in Eq. (B.2) can be solved as 


$$
C_{k, i}(s)=C_{k, i}(0) e^{-\lambda_{k} s}+\lambda \int_{0}^{s} \psi_{k, i}\left(s^{\prime}\right) e^{-\lambda_{k}\left(s-s^{\prime}\right)} d s^{\prime}
$$

In Eq. (B.5), $C_{k, i}(0)=C_{k, i}\left(z_{0}, t_{0}\right)$ is the initial or boundary condition given at the starting point of the characteristic line. For example, as illustrated in Fig. B.1, the precursor concentration in an axial node $j$ with an interval $\left[z_{j-1}, z_{j}\right]$ during a time interval $\left[t_{n-1}, t_{n}\right]$ can be obtained starting from the initial condition $C_{k, i j}^{n-1}(z), z_{j-1} \leq z \leq z_{j}$ given at $t=t_{n-1}$ or the inlet boundary condition $C_{k, i j}^{i n}(t), t_{n-1} \leq t \leq t_{n}$ given at $z=z_{j-1}$.

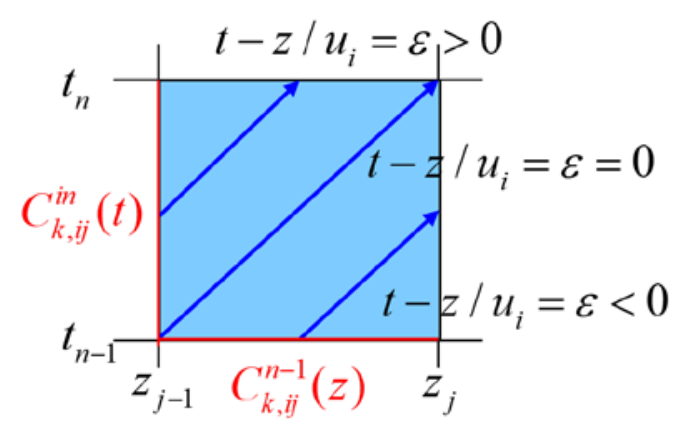

Fig. B.1. Illustration of characteristic lines for precursor concentration calculation.

Along a characteristic line starting from the line $t=t_{n-1}, z_{0}=z-u_{i}\left(t-t_{n-1}\right)>z_{j-1}$ and thus $t-t_{n-1}<\left(z-z_{j-1}\right) / u_{i}$. On the other hand, along a characteristic line starting from the line $z=z_{j-1}, t_{0}=t-\left(z-z_{j-1}\right) / u_{i}>t_{n-1}$ and thus $t-t_{n-1}>\left(z-z_{j-1}\right) / u_{i}$. Therefore, the precursor concentration in the axial node $j$ during a time interval $\left[t_{n-1}, t_{n}\right]$ can be determined as $C_{k, i}(z, t)=\left\{\begin{array}{l}C_{k, i j}^{i n}(t) e^{-\lambda_{k} \frac{z-z_{j-1}}{u_{i}}}+\frac{\lambda}{u_{i}} \int_{z_{j-1}}^{z} \psi_{k, i}\left[z^{\prime}, t-\frac{z-z^{\prime}}{u_{i}}\right] e^{-\lambda_{k} \frac{z-z^{\prime}}{u_{i}}} d z^{\prime}, \quad t-t_{n-1}>\frac{z-z_{j-1}}{u_{i}} \\ C_{k, i j}^{n-1}(z) e^{-\lambda_{k}\left(t-t_{n-1}\right)}+\frac{\lambda}{u_{i}} \int_{z-u_{i}\left(t-t_{n-1}\right)}^{z} \psi_{k, i}\left[z^{\prime}, t-\frac{z-z^{\prime}}{u_{i}}\right] e^{-\lambda_{k} \frac{z-z^{\prime}}{u_{i}}} d z^{\prime}, t-t_{n-1}<\frac{z-z_{j-1}}{u_{i}}\end{array}\right.$

At each time node, the precursor concentrations at the outgoing surface of each node are determined using Eq. (B.6). The precursor production and decay during the time interval $\Delta t_{n}$ are evaluated along the characteristic line from a starting point $z_{\text {start }}^{n}$ to an ending point at 
$Z=Z_{\text {out }}$. For an ending point at $Z=z_{\text {out }}$ at $t=t_{n}$, the starting point at $t=t_{n-1}$ can be determined as

$$
z_{\text {start }}^{n}=z_{\text {out }}-u_{i} \Delta t_{n}
$$

The precursor concentration at the starting position is determined by the linear interpolation of precursor concentrations at the previous time node as

$$
C_{k, i}\left(z_{\text {start }}^{n}\right)=\gamma C_{k, i}^{n-1}\left(z_{\text {in }}\right)+(1-\gamma) C_{k, i}^{n-1}\left(z_{\text {out }}\right),
$$

where $Z_{\text {in }}$ is the inlet coordinate of the node of interest and

$$
\gamma=\left(z_{\text {out }}-z_{\text {start }}^{n}\right) /\left(z_{\text {out }}-z_{\text {in }}\right) \text {. }
$$

In order to evaluate the precursor generation rate in Eq. (B.6), the unknown flux at the current time node $t_{n}$ is approximated by assuming the flux varies exponentially in the time interval $\left[t_{n-1}, t_{n}\right]$ as

$$
\phi_{g, i j}(z, t)=\phi_{g, i j}^{n-1}(z) e^{\alpha_{n}\left(t-t_{n-1}\right)} .
$$

The inverse period $\alpha_{n}$ is approximately determined using the power levels at $t_{n-2}$ and $t_{n-1}$ as

$$
\alpha_{n}=\frac{1}{\Delta t_{n-1}} \ln \frac{P_{n-1}}{P_{n-2}} \text {. }
$$

Fig. B.2 illustrates the method to calculate the precursor concentration at $t=t_{n}$ on the outlet surface of a node $j$ (i.e., at $z=z_{j}$ ). If the fuel salt moves through $L$ nodes during a time interval $\left[t_{n-1}, t_{n}\right]$, then starting from the node $j-(L-1)$, the outgoing precursor concentrations of the nodes between $z_{\text {start }}^{n}$ to $z_{j}$ are calculated as 


$$
C_{k, i, j-l}^{n}=\left\{\begin{array}{l}
C_{k, i}\left(z_{s t a r t}^{n}\right) e^{-\lambda_{k} \frac{z_{\text {out }}-z_{\text {start }}}{u_{i, j-l}^{n}}}+\frac{\lambda}{\bar{u}_{i, j-l}^{n}} \int_{z_{\text {start }}^{n}}^{z_{j-l}} \psi_{k, i, j-l}^{n-1}\left(z^{\prime}\right) e^{-\left(\lambda_{k}+\alpha_{n}\right) \frac{z_{j-l}-z^{\prime}}{\bar{u}_{i, j-l}^{n}}} d z^{\prime}, z_{j-l-1}<z_{\text {start }}^{n} \quad, \quad l=1, \cdots, L-1 \\
C_{k, i, j-l-1} e^{-\lambda_{k} \frac{z_{\text {out }}-z_{\text {start }}}{\bar{u}_{i, j-l}^{n}}}+\frac{\lambda}{\bar{u}_{i, j-l}^{n}} \int_{z_{j-l-1}}^{z_{j-l}} \psi_{k, i, j-l}^{n-1}\left(z^{\prime}\right) e^{-\left(\lambda_{k}+\alpha_{n}\right) \frac{z_{j-l}-z^{\prime}}{\bar{u}_{i, j-l}^{n}}} d z^{\prime}, \quad z_{j-l-1}>z_{\text {start }}^{n}
\end{array}\right.
$$

where $\bar{u}_{i, j}^{n}$ is the average fuel velocity during the time step $\left[t_{n-1}, t_{n}\right]$ in an axial node $j$ in a radial mesh $i$, and the outgoing concentrations from a node are used as the incoming concentrations for the next node.

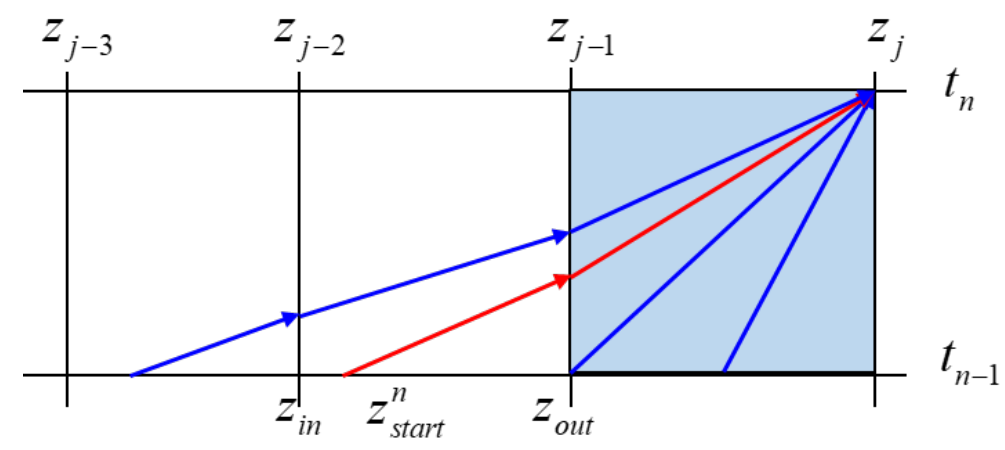

Fig. B.2. Fuel salt path at each time step from a starting point to a node outlet.

With this method, the precursor concentrations at $t=t_{n}$ are calculated using the precursor concentrations at $t=t_{n-1}$ and the precursor generation rates extrapolated from the power levels at the previous two time nodes. It was observed that this method could be unstable for low velocities with which the fuel salt can move only a small fraction of a node during a time interval.

\section{B.1.2. Method of Lines}

In this method, the precursor equation in Eq. (3.11) is solved semi-analytically by converting the PDE to a system of ODEs and by solving the resulting system of ODEs using a proper ODE solver. This can be done by discretizing one of the derivatives of Eq. (3.11) and solving the system of ODEs for the remaining variable. The most important advantage of this MoL approach comes from the fact that ODE is generally simpler to solve than PDE and the implicit methods can be used for discretization. In this study, the time derivative is discretized with the backward Euler (implicit) method as 


$$
\frac{\partial}{\partial t} C_{k, i}(z, t)=\frac{C_{k, i}^{n}(z)-C_{k, i}^{n-1}(z)}{\Delta t_{n}}, \quad \Delta t_{n}=t_{n}-t_{n-1} .
$$

By inserting Eq. (B.13) into Eq. (3.11), the following equation can be obtained for each radial node $i$ :

$$
\frac{d}{d z}\left[u_{i}^{n}(z) C_{k, i}^{n}(z)\right]+\left(\lambda_{k}+\frac{1}{\Delta t_{n}}\right) C_{k, i}^{n}(z)=\lambda \psi_{k, i}^{n}(z)+\frac{1}{\Delta t_{n}} C_{k, i}^{n-1}(z) .
$$

The total delayed neutron precursor source at the right hand side is related to both the delayed neutron precursor source at the current time step and the delayed neutron precursor concentration at the previous time step. The time-dependent delayed precursor equation at each time step can be written in a more convenient form to solve as

$$
\frac{d}{d z}\left[u_{i}^{n}(z) C_{k, i}^{n}(z)\right]+\frac{\lambda_{k}^{n}}{u_{i}^{n}(z)}\left[u_{i}^{n}(z) C_{k, i}^{n}(z)\right]=Q_{k, i}^{n}(z),
$$

where

$$
\lambda_{k}^{n}=\lambda_{k}+\frac{1}{\Delta t_{n}}, \quad Q_{k, i}^{n}(z)=\lambda \psi_{k, i}^{n}(z)+\frac{1}{\Delta t_{n}} C_{k, i}^{n-1}(z) .
$$

Instead of using an ODE solver, Eq. (B.15) can solved analytically for the delayed neutron precursor concentration in terms of the inlet precursor concentration and the precursor concentration at the previous time step as

$$
u_{i}^{n}(z) C_{k, i}^{n}(z)=e^{-\lambda_{k}^{n} \int_{z_{0}}^{z} \frac{d z_{i}^{\prime}}{u_{i}^{n}\left(z^{\prime}\right)}}\left[u_{i}^{n}\left(z_{0}\right) C_{k, i}^{n}\left(z_{0}\right)+\int_{z_{0}}^{z} d z^{\prime} Q_{k, i}^{n}\left(z^{\prime}\right) e^{\lambda_{k}^{n} \int_{z_{0}}^{z^{\prime}} \frac{d z_{i}^{n}\left(z^{\prime \prime}\right)}{1}}\right] \text {. }
$$

Then, the outgoing and average precursor concentrations of an axial node $j$ at a time node $n$ can be obtained as

$$
\begin{aligned}
& C_{k, i j}^{n}=\frac{u_{i, j-1}^{n}}{u_{i j}^{n}} e^{-\lambda_{k}^{n} \Delta t_{i j}^{n}} C_{k, i, j-1}^{n}+\frac{\bar{u}_{i j}^{n}}{u_{i j}^{n} \lambda_{k}^{n}} Q_{k, i j}^{n}\left[1-e^{-\lambda_{k}^{n}\left(z-z_{j-1}\right) / u_{i j}^{n}}\right], \\
& \bar{C}_{k, i j}^{n}=\frac{u_{i, j-1}^{n}}{u_{i j}^{n}} \frac{1}{\lambda_{k}^{n} \Delta t_{i j}^{n}}\left[1-e^{-\lambda_{k}^{n} \Delta t_{i j}^{n}}\right] C_{k, i, j-1}^{n}+\frac{1}{\lambda_{k}^{n}} Q_{k, i j}^{n}\left\{1-\frac{1}{\lambda_{k}^{n} \Delta t_{i j}^{n}}\left[1-e^{-\lambda_{k}^{n} \Delta t_{i j}^{n}}\right]\right\} .
\end{aligned}
$$

The node-averaged precursor concentration in Eq. (B.19) depends on the fission rate at the end of a time step. In the MoL method, the fission rates are determined iteratively by coupling Eq. (B.19) with the TFSP at the end of a time step, while in the MOC approach, 
they were determined approximately with an assumed exponential flux shape with an inverse period determined by extrapolating the power levels of two previous time nodes. However, the time discretization error introduces some errors in the initial precursor concentrations, and this could lead to an instability issue.

\section{B.2. Comparison of Transient Solutions}

The accuracy of the three solution approaches, FDM, MOC, and MoL were examined by solving a null transient problem and the pump startup and coast down transient problems without thermal feedback. For the latter two transients, the fuel velocity was increased or decreased exponentially with a time constant of $1 \mathrm{~s}$, and a fuel salt transient time of $4 \mathrm{~s}$ ( $2 \mathrm{~s}$ in the core and $2 \mathrm{~s}$ outside of the core) was used.

Fig. B.3 compares the power evolutions during the first $10 \mathrm{~s}$ obtained with the three solution methods for a null transient. It is clearly seen that the FDM is stable and produces the steady state solution accurately. However, the MOC and MoL power solutions show diverging behaviors due to error accumulation although the magnitudes are very small. The MoL power solution increases steadily while the MOC power solution decreases. It appears that these unstable behaviors are due to the approximations introduced in MOC and MoL that do not reproduce the initial steady state precursor concentrations.

The power response to the change of the fuel salt flow rate due to the pump startup and pump coast-down transients are shown in Fig. B.4 and Fig. B.5, respectively. The pump startup introduces a negative reactivity due to the increased delayed neutron fraction decaying outside of the core, and thus the power decreases with time in an oscillatory way as shown in Fig. B.4. Relative to the FDM solution, the MOC and MoL solutions show oscillatory errors. The MOC solution error varies from about $-6 \%$ to $~ 3 \%$, but it can be seen that the amplitude decreases with time. The MoL solution error varies from about $-3 \%$ to $\sim 3 \%$, and its amplitude initially increases but decreases after $\sim 20 \mathrm{~s}$. The pump coast-down introduces a positive reactivity and thus the power increases exponentially as shown in Fig. B.5. It can be seen that both the MOC and MoL solution errors increase monotonically in the opposite direction as in the null transient case shown in Fig. B.3. 


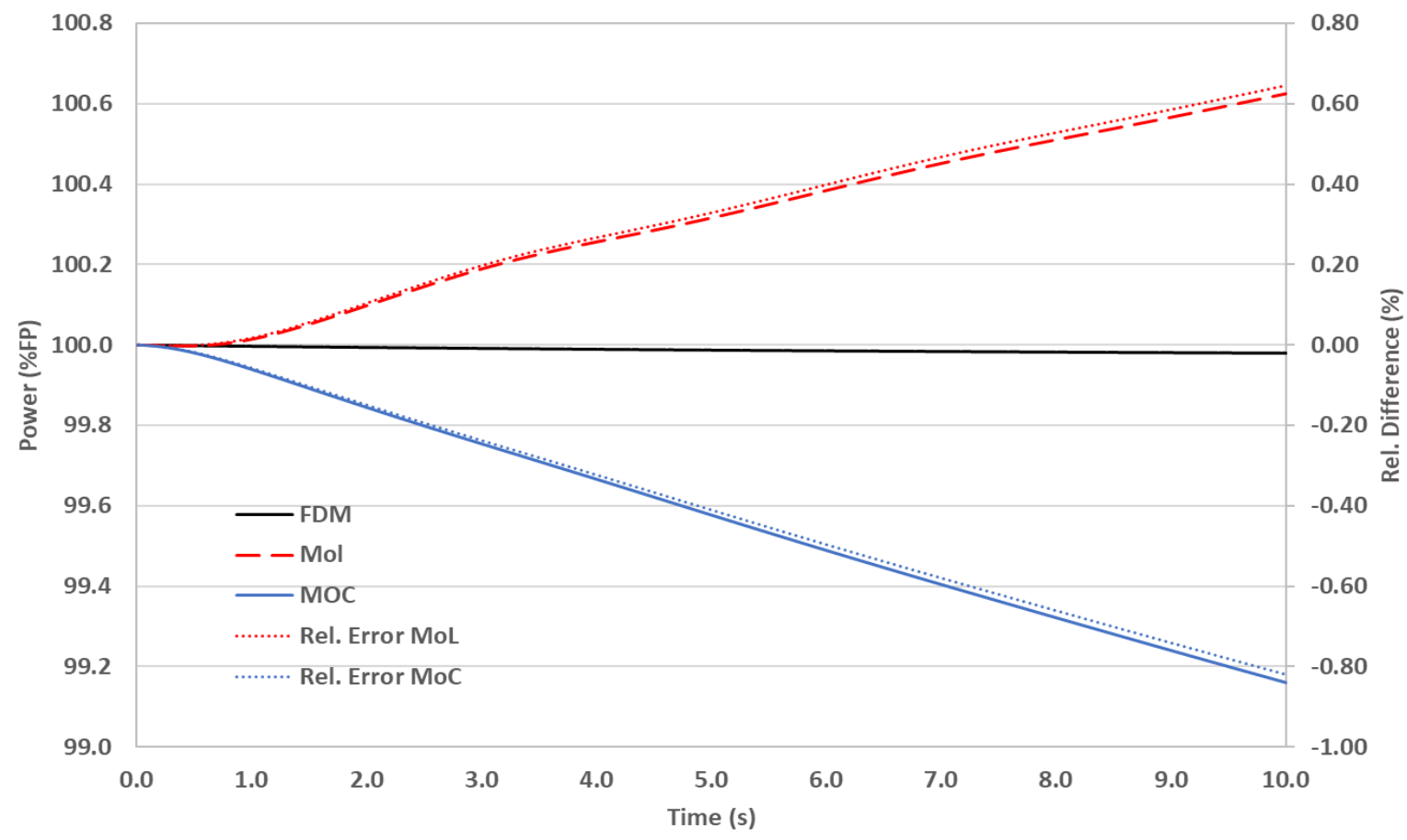

Fig. B.3. Power evolution in null transient without thermal feedback.

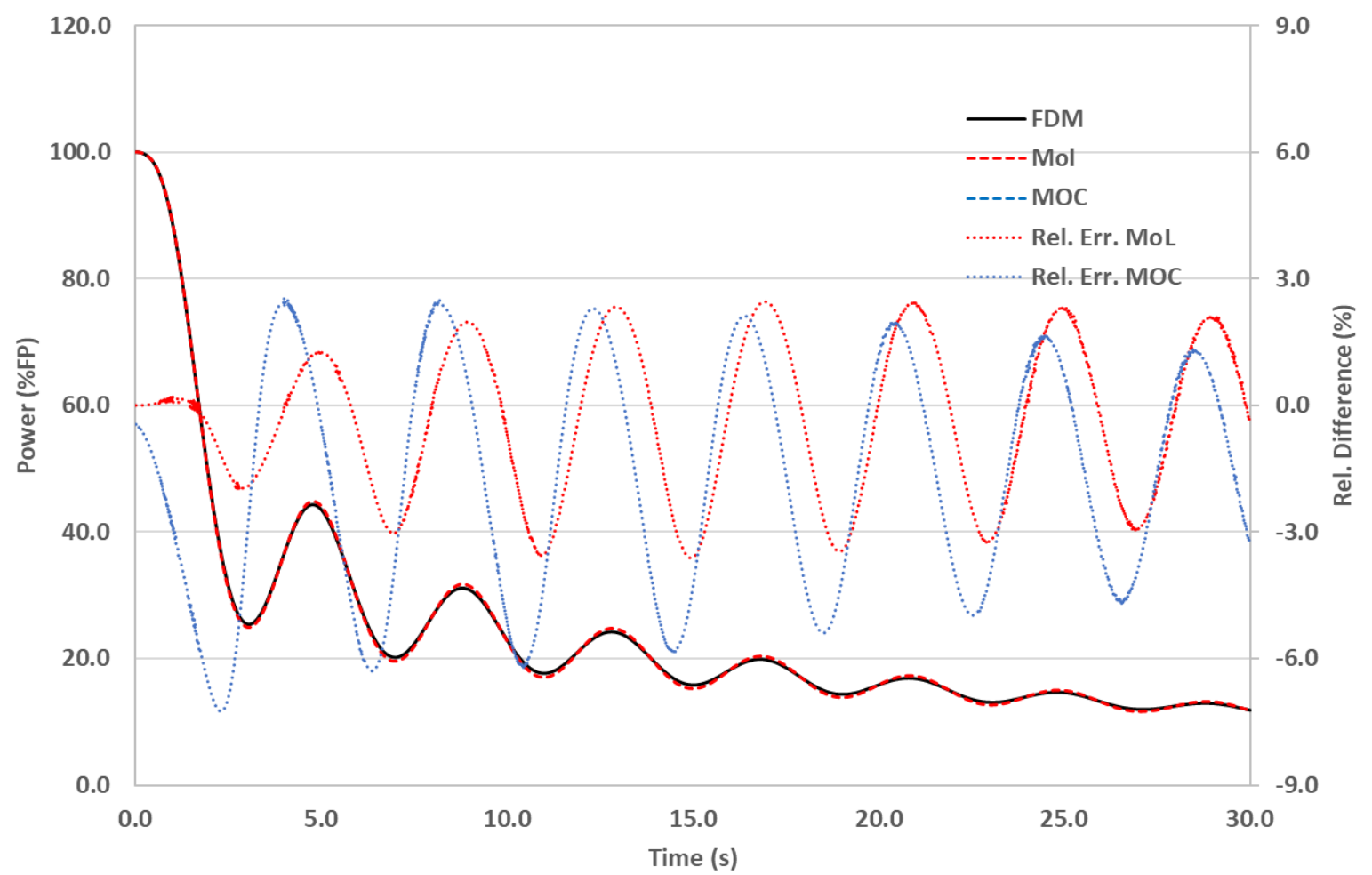

Fig. B.4. Power evolution in pump start-up transient without thermal feedback. 
Multiphysics Coupling of PROTEUS-NODAL and SAM for Molten Salt Reactor Simulation February 28, 2020

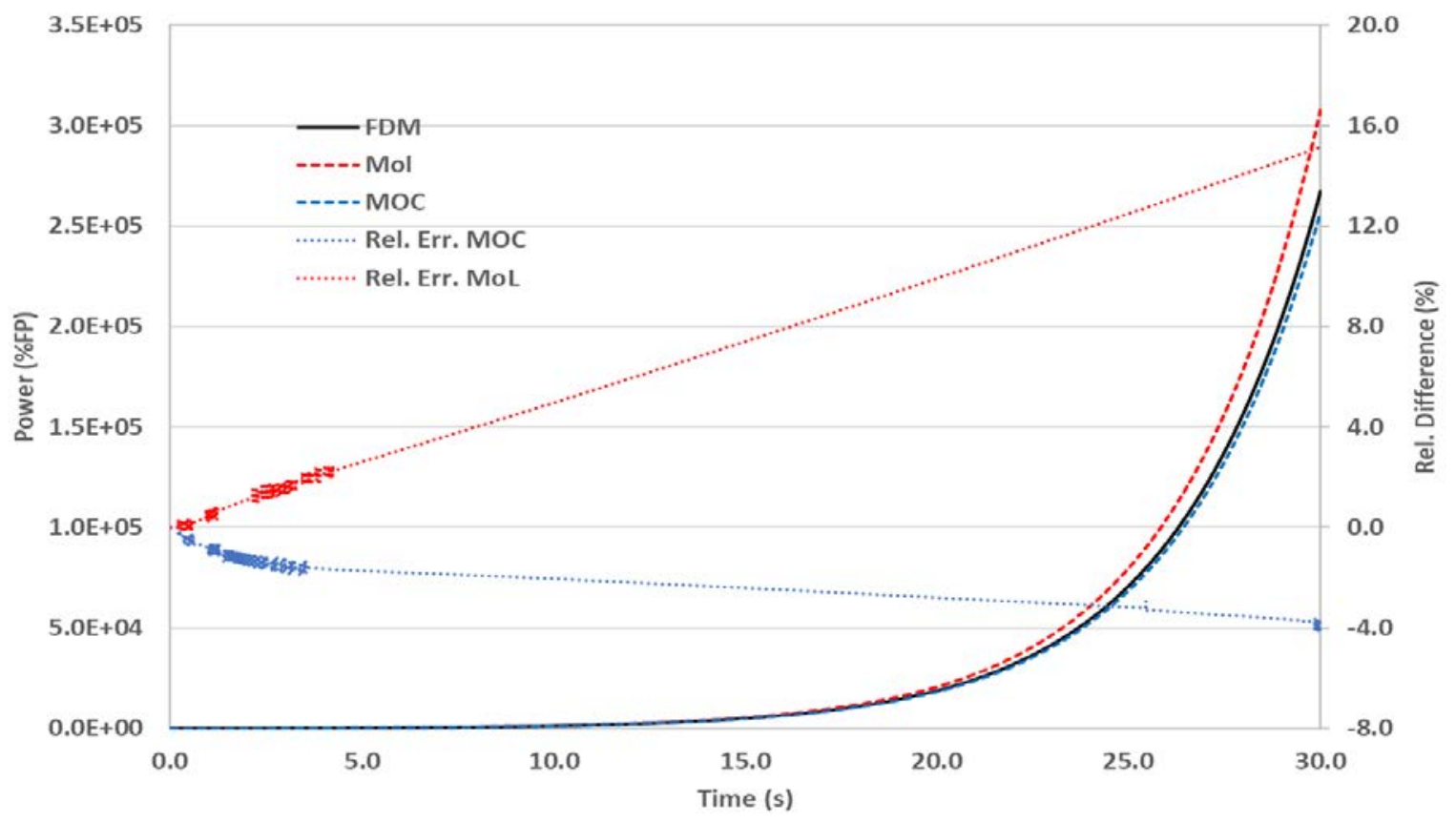

Fig. B.5. Power evolution in pump coast-down transient without thermal feedback. 


\section{Appendix C. Standalone MSR Thermal-Hydraulics Solver of PROTEUS}

In order to account for thermal feedback effects, a single-phase, parallel-channel thermalhydraulics model was added to PROTEUS-NODAL. In this model, the thermal-fluidic behavior of the core is described by representing the core by one-dimensional parallel channels. For a thermal MSR with solid moderator, a given total flow rate is split among the parallel flow channels to satisfy the equal pressure drop boundary conditions. Meanwhile, for a fast spectrum MSR, a uniform mass flow rate is applied at the core inlet without considering flow splitting.

\section{C.1. Governing Equations}

Ignoring the axial heat conduction and the shear forces due to velocity gradients in the fuel salt, the mass, momentum, and energy balance equations for one-dimensional flow in a vertical channel can be written as [29]

$$
\begin{gathered}
\frac{\partial \rho}{\partial t}+\frac{\partial(\rho u)}{\partial z}=0, \\
\frac{\partial(\rho u)}{\partial t}+\frac{\partial}{\partial z}\left(\rho u^{2}\right)=-\frac{\partial P}{\partial z}-\rho g-\left(\frac{\partial P}{\partial z}\right)_{\text {fric }}, \\
\frac{\partial(\rho h)}{\partial t}+\frac{\partial(\rho u h)}{\partial z}=q_{\text {salt }}^{\prime \prime \prime}+\frac{q^{\prime \prime} P_{h}}{A} .
\end{gathered}
$$

where $\rho, u, h$, and $P$ are the density, velocity, temperature, and pressure of the fuel salt, respectively, $q_{\text {salt }}^{\prime \prime \prime}$ is the volumetric heat source produced in the fuel salt, $q^{\prime \prime}$ is the surface heat flux, $P_{h}$ and $A$ are the wetted perimeter and flow area of fuel salt channel, and $g$ is the gravitational acceleration. The friction pressure gradient is related to the wall shear stress and the momentum flux as

$$
\left(\frac{\partial P}{\partial z}\right)_{\text {fric }}=-\frac{\tau_{w} P_{h}}{A}=\frac{f \rho u^{2}}{2 D_{h}},
$$

where $\tau_{w}$ is the wall shear stress, $D_{h}$ is the hydraulic diameter, and $f$ is the friction factor for a smooth tube and all flow regimes, which is obtained from Blasius and Petukhov [30] 
relations for Darcy friction factor as a function of Reynolds number for smooth pipes

$$
f=\left\{\begin{array}{cc}
0.316 \mathrm{Re}^{-0.25} & \operatorname{Re}<3000 \quad \text { (Blasius relation) } \\
\frac{1.0}{(1.82 \ln (\mathrm{Re})-1.64)^{2}} & 3000<\mathrm{Re}<10^{6} \text { (Petukhov relation) }
\end{array},\right.
$$

where Re is the Reynolds number defined as

$$
\operatorname{Re}=\frac{\rho u D_{h}}{\mu} .
$$

The momentum and energy equations can be simplified by combining each with the continuity equation in Eq. (C.1) as

$$
\begin{gathered}
\rho \frac{\partial u}{\partial t}+\rho u \frac{\partial u}{\partial z}=-\frac{\partial P}{\partial z}-\rho g-\left(\frac{\partial P}{\partial z}\right)_{\text {fric }}, \\
\rho \frac{\partial h}{\partial t}+\rho u \frac{\partial h}{\partial z}=q_{\text {salt }}^{\prime \prime \prime}+\frac{\partial P}{\partial t}+u\left[\frac{\partial P}{\partial z}+\left(\frac{\partial P}{\partial z}\right)_{\text {fric }}\right],
\end{gathered}
$$

The thermodynamic relation for the enthalpy change can be written as

$$
d h=\left.\frac{d h}{d T}\right|_{P} d T+\left.\frac{d h}{d P}\right|_{T} d P=c_{p} d T+\frac{1-\beta T}{\rho} d P,
$$

Since the energy variation due to pressure changes and frictional dissipation is usually negligible compared to the heat addition and enthalpy change terms, Eq. (C.9) can be simplified as

$$
d h=\left.\frac{d h}{d T}\right|_{P} d T=c_{p} d T .
$$

Since the material properties of the fuel salt depend on the temperature only, it is convenient to write the energy balance equation Eq. (C.8) in terms of temperature using Eq. (C.10) as

$$
\rho c_{p} \frac{\partial T}{\partial t}+\rho u c_{p} \frac{\partial T}{\partial z}=q_{s a l t}^{\prime \prime \prime}+\frac{q^{\prime \prime} P_{h}}{A} .
$$

For the boundary conditions of these conservation equations, the inlet flow rate, the inlet enthalpy, and the outlet pressure can be used. These boundary conditions are determined 
using the given system outlet pressure and total salt flow rate. In the steady state, the time derivatives of Eq. (C.1), Eq. (C.7), and Eq. (C.11) are zero. In this case, the mass flux $\rho u$ is constant, and hence Eq. (C.7) and Eq. (C.11) can be integrated separately as:

$$
\begin{gathered}
T(z)=T_{\text {in }}+\frac{1}{\rho u c_{p} A} \int_{0}^{z} q_{\text {salt }}^{\prime}\left(z^{\prime}\right) d z^{\prime}+\frac{P}{\rho u c_{p} A} \int_{0}^{z} q^{\prime \prime}\left(z^{\prime}\right) d z^{\prime}, \\
P(z)=P_{\text {in }}+(\rho u)^{2}\left[\frac{1}{\rho_{\text {in }}}-\frac{1}{\rho(z)}\right]-g \int_{0}^{z} \rho\left(z^{\prime}\right) d z^{\prime}-\frac{(\rho u)^{2}}{2 D_{h}} \int_{0}^{z} \frac{f\left(z^{\prime}\right)}{\rho\left(z^{\prime}\right)} d z^{\prime} .
\end{gathered}
$$

Since $\rho$ is generally a function of $T$ and $P$, Eq. (C.12) represents a non-linear equation for $P(z)$. If $\rho$ is independent of $P$ (i.e., incompressible), $\rho(z)$ can be determined with $T(z)$ obtained from Eq. (C.7), hence Eq. (C.13) is solved by simple integrations.

Neglecting the axial conduction, the heat conduction in the annual moderator ring can be written as

$$
\frac{\partial}{\partial t}\left[\rho_{m} c_{p, m} T_{m}(r, z, t)\right]=\frac{1}{r} \frac{\partial}{\partial r}\left[k_{m} r \frac{\partial}{\partial r} T_{m}(r, z, t)\right]+q_{m}^{\prime \prime \prime}(r, z, t),
$$

where $\rho_{m}, c_{p, m}, T_{m}$ are the solid moderator density, specific heat, and temperature, respectively, $q_{m}^{\prime \prime \prime}$ is the volumetric heat source generated in the solid moderator due to gamma heating, and $k_{m}$ is the heat conduction coefficient of the solid moderator. Fig. C.1 shows a schematic configuration of the fuel salt and solid moderator unit cell.

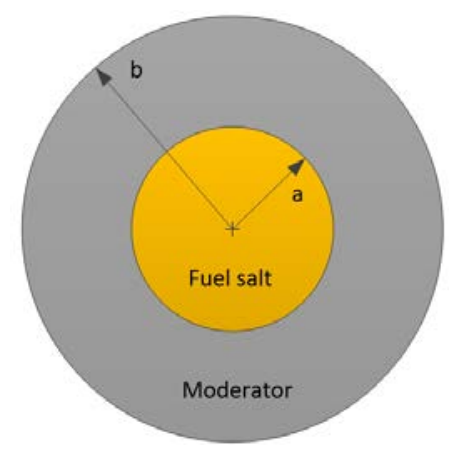

Fig. C.1. Radial configuration of unit cell. 
The heat conduction equation for annular moderator is coupled with the fuel salt energy equation in Eq. (C.3) or Eq. (C.11). The moderator temperature at the moderator inner wall is coupled with the bulk fuel salt temperature through the Newton's law for heat convection

$$
\left.k_{m} \frac{\partial T_{m}(r, z, t)}{\partial r}\right|_{r=a}=h_{f}\left[T_{m}(a, z, t)-T_{f}(z, t)\right]=q^{\prime \prime}(z, t)
$$

The heat transfer coefficient $h_{f}$ is calculated as

$$
h_{f}=\frac{k_{f}}{L} \mathrm{Nu}
$$

where $L$ is the characteristic length of the fuel channel, $k_{f}$ is the heat conduction coefficient of the fuel salt, and $\mathrm{Nu}$ is the Nusselt number given by

$$
N u=\left\{\begin{array}{ll} 
\begin{cases}4.36, & \operatorname{Re} \leq 2300 \\
0.023 \mathrm{Re}^{0.8} \mathrm{Pr}^{0.4}, & \mathrm{Re}>2300\end{cases} \\
\left\{\begin{array}{ll}
0.59 \mathrm{Ra}^{0.25}, & \mathrm{Re} \leq 2300 \\
0.10 \mathrm{Ra}^{1 / 3}, & \mathrm{Re}>2300
\end{array}\right. \text { for forced convection }
\end{array} \text { for natural convection } .\right.
$$

In this expression, Re is the Reynolds number defined in Eq. (C.6), and Pr and Ra are the Prandtl and the Rayleigh numbers, respectively, defined as

$$
\begin{gathered}
\operatorname{Pr}=\frac{c_{p, f} \mu_{f}}{k_{f}}, \\
\operatorname{Ra}=\mathrm{Gr} \cdot \operatorname{Pr}=\frac{c_{p, f} \rho_{f}^{2} g \beta\left[T_{m}(a)-\bar{T}_{f}(z)\right] L^{3}}{k_{f} \mu_{f}},
\end{gathered}
$$

where $\rho_{f}, \mu_{f}$, and $\beta$ are the density, dynamic viscosity, and temperature expansion coefficient of fuel salt, respectively, and Gr is the Grashof number defined as

$$
\mathrm{Gr}=\frac{\rho_{f}^{2} g \beta\left[T_{m}(a)-\bar{T}_{f}(z)\right] L^{3}}{\mu_{f}^{2}} .
$$


The other boundary condition for the heat conduction in moderator is given by the symmetry condition at the outer boundary of the moderator

$$
\left.\frac{\partial T_{m}(r, z, t)}{\partial r}\right|_{r=b}=0
$$

The fuel salt temperatures in the lower and upper plena are assumed equal to the fuel salt inlet and outlet temperatures, respectively. This yields the following boundary conditions

$$
\begin{gathered}
T_{f}(r, 0, t)=T_{\text {in }}(t), \\
T_{f}(r, H, t)=T_{\text {out }}(t),
\end{gathered}
$$

where $T_{\text {in }}$ is the fuel salt temperature at the core inlet and $T_{\text {out }}$ is the fuel salt temperature at the core outlet. The boundary conditions in Eq. (C.15), Eq. (C.21), Eq. (C.22), and Eq. (C.23) can be represented by a general mixed boundary condition

$$
\alpha \vec{n} \cdot k \nabla T+\beta T=\gamma,
$$

where $\vec{n}$ is the outward normal vector of the boundary surface. That is, $\alpha=1, \beta=-h_{f}$ and $\gamma=-h_{f} T_{f}$ for Eq. (C.15), $\alpha=1$ and $\beta=\gamma=0$ for Eq. (C.21), $\alpha=0, \beta=1$ and $\gamma=T_{\text {in }}$ for Eq. (C.22), and $\alpha=0, \beta=1$ and $\gamma=T_{\text {out }}$ for Eq. (C.23).

\section{C.2. Spatial Discretization}

The control volume approach is adopted in this study, where the problem domain is divided into computational cells in the axial direction. Each grid point is placed at the geometric center of each cell. Field variables such as density, pressure, and temperature are defined at these grid points, and the flow variables such as velocity component and mass flux are defined on cell faces. The main control volumes for mass and energy balances are defined by these cells as shown in Fig. C.2. The momentum control volume is displaced from the main control volume in the velocity direction, extending from grid points to grid points and encompassing the cell face upon which the velocity component is defined. The finite difference equations are derived by integrating the mass, momentum, and energy equations over the control volumes under the assumption that the values at the center of each control volume prevail over the control volume. 


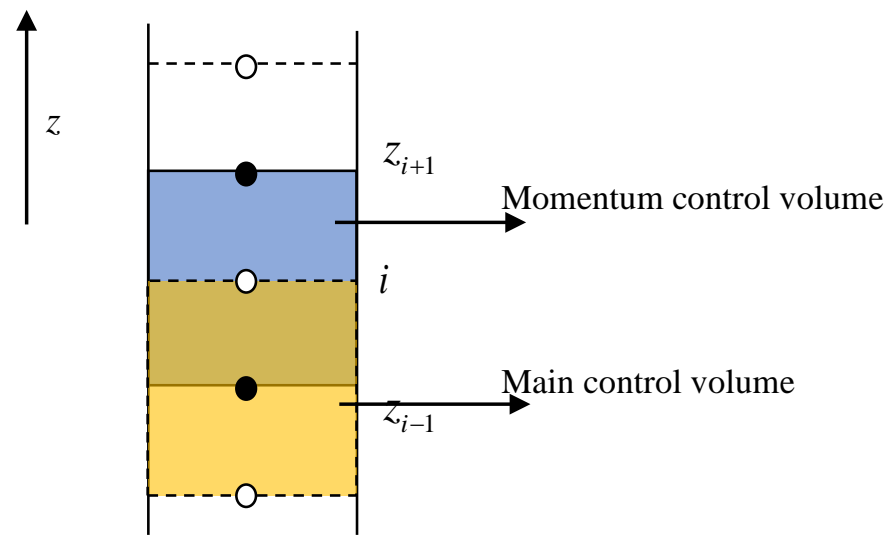

Fig. C.2. Main and momentum control volumes and state variables.

The mass and energy balance equations can be obtained by integrating Eq. (C.1) and Eq. (C.11), respectively, over the main control volume $i\left(z_{i-1} \leq z \leq z_{i}\right)$ and representing the fuel salt densities and temperatures at cell faces with the cell-center values. The momentum balance equation for the cell is obtained by integrating Eq. (C.7) over the momentum control volume ( $z_{i-1}+\Delta z_{i} / 2 \leq z \leq z_{i}+\Delta z_{i+1} / 2$ ). Using the up-wind differencing scheme for upward flow in a constant flow area, the spatially discretized forms of the mass, energy, and momentum conservation equations are obtained as

$$
\begin{gathered}
\frac{\partial \bar{\rho}_{i}}{\partial t}=\frac{\bar{\rho}_{i-1} u_{i-1}-\bar{\rho}_{i} u_{i}}{\Delta z_{i}}, \\
\frac{\partial \bar{T}_{i}}{\partial t}=\frac{\bar{\rho}_{i-1} u_{i-1}}{\bar{\rho}_{i}} \frac{\bar{T}_{i-1}-\bar{T}_{i}}{\Delta z_{i}}+\frac{\bar{q}_{i}}{\bar{c}_{p, i} \bar{\rho}_{i} A_{i} \Delta z_{k}}+\frac{q_{i}^{\prime \prime} P_{h, i}}{\bar{c}_{p, i} \bar{\rho}_{i} A_{i}}, \\
\frac{d}{d t}\left(\bar{\rho}_{i} u_{i}\right)=\frac{2}{\Delta z_{i}+\Delta z_{i+1}}\left(\frac{\bar{\rho}_{i-1}^{2}}{\bar{\rho}_{i}} u_{i-1}^{2}-\frac{\bar{\rho}_{i}^{2}}{\bar{\rho}_{i+1}} u_{i}^{2}\right)+\frac{2}{\Delta z_{i}+\Delta z_{i+1}}\left(\overline{P_{i}}-\bar{P}_{i+1}\right) \\
-\frac{\left(\bar{\rho}_{i} \Delta z_{i}+\bar{\rho}_{i+1} \Delta z_{i+1}\right)}{\Delta z_{i}+\Delta z_{i+1}} g-\frac{f_{i} \bar{\rho}_{i} u_{i}^{2}}{2 D_{h}} .
\end{gathered}
$$

where $\rho_{i}=\rho\left(\bar{P}_{i}, \bar{h}_{i}\right)=\bar{\rho}_{i}$ and $\bar{T}_{i}$ are the fuel salt density and temperature, respectively, at the cell center, $\bar{q}_{i}$ is the cell-averaged heat source, $u_{i-1}$ and $u_{i}$ are the velocities at the inlet and 
outlet faces of cell $i$, respectively, and $\Delta z_{i}=z_{i}-z_{i-1}$. Using Eq. (C.25) and Eq. (C.27), the momentum balance equation can be reduced to

$$
\begin{aligned}
\frac{d u_{i}}{d t}= & \frac{\bar{\rho}_{i-1} u_{i-1}}{\bar{\rho}_{i}}\left(\frac{2}{\Delta z_{i}+\Delta z_{i+1}} \frac{\bar{\rho}_{i-1}}{\bar{\rho}_{i}} u_{i-1}-\frac{u_{i}}{\Delta z_{i}}\right)-u_{i}^{2}\left(\frac{2}{\Delta z_{i}+\Delta z_{i+1}} \frac{\bar{\rho}_{i}}{\bar{\rho}_{i+1}}-\frac{1}{\Delta z_{i}}\right) \\
& +\frac{2}{\bar{\rho}_{i}\left(\Delta z_{i}+\Delta z_{i+1}\right)}\left(\bar{P}_{i}-\bar{P}_{i+1}\right)-\frac{\left(\bar{\rho}_{i} \Delta z_{i}+\bar{\rho}_{i+1} \Delta z_{i+1}\right)}{\bar{\rho}_{i}\left(\Delta z_{i}+\Delta z_{i+1}\right)} g-\frac{f_{i} u_{i}^{2}}{2 D_{h}}
\end{aligned}
$$

where $\bar{P}_{i}$ and $\bar{P}_{i+1}$ are the pressure at the center of the cell $i$ and $i+1$, respectively. For the cell at the top of the channel, Eq. (C.27) is reduced to

$$
\frac{d u_{I}}{d t}=\frac{1}{\Delta z_{I}} \frac{\bar{\rho}_{I-1} u_{I-1}}{\bar{\rho}_{I}}\left(2 \frac{\bar{\rho}_{I-1}}{\bar{\rho}_{I}} u_{I-1}-u_{I}\right)-\frac{u_{I}^{2}}{\Delta z_{I}}+\frac{2}{\bar{\rho}_{I} \Delta z_{I}}\left(\bar{P}_{I}-P_{\text {out }}\right)-g-\frac{f_{I} u_{I}^{2}}{2 D_{h}} .
$$

where $P_{\text {out }}$ is the outlet pressure.

For the heat conduction equation, the annular moderator is divided into computational cells, where the temperatures are defined at the cell center as shown in Fig. C.3. The finite difference equations can be derived by integrating the heat conduction equation over each cell volume under the assumption that the value at the cell center prevail over the cell volume.

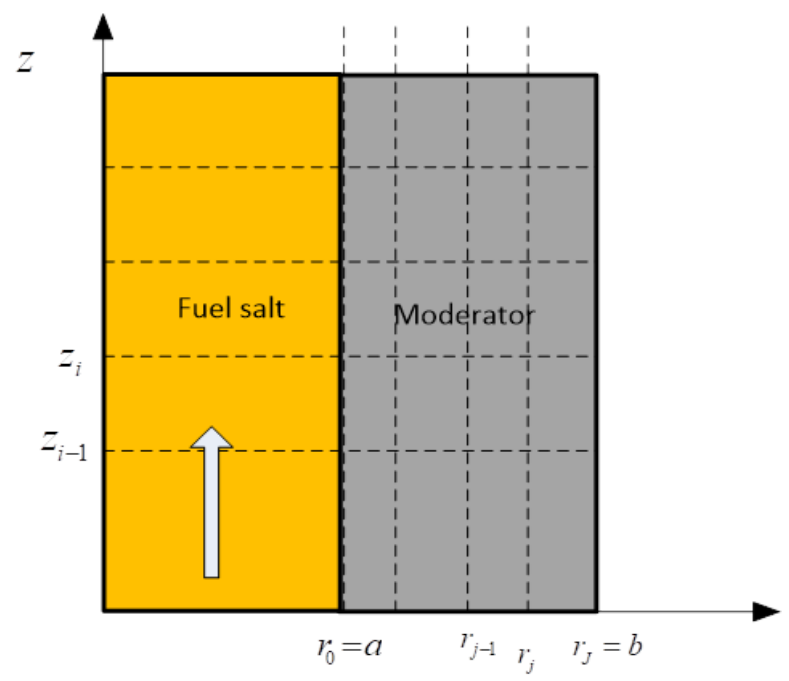

Fig. C.3. Mesh structure for heat conduction calculations. 
Consider a cell $(i, j)$ with $z_{i-1} \leq z \leq z_{i}$ and $r_{j-1} \leq r \leq r_{j}$. Integrating Eq. (C.14) over the cell volume, we have

$$
\begin{gathered}
\frac{d}{d t}\left(\rho_{m, i j} C_{m, p, i j} \bar{T}_{m, i j}\right) V_{i j}=\left[\begin{array}{l}
2 \pi r_{j} k_{m, i j} \frac{\partial}{\partial r}\left[\frac{1}{\Delta z_{i}} \int_{z_{i-1}}^{z_{i}} T_{m}(r, z, t) d z\right] \\
-\left.2 \pi r_{j-1} \frac{\partial}{\partial r}\left[\frac{1}{\Delta z_{i}} \int_{z_{i-1}}^{z_{i}} T_{m}(r, z, t) d z\right]\right|_{r=r_{j}}
\end{array}\right] \Delta z_{i}+\bar{q}_{m, i j}^{\prime \prime \prime} V_{m, i j}, \\
\frac{d}{d t}\left(\rho_{m, i j} c_{m, p, i j} \bar{T}_{m, i j}\right) V_{m, i j}=\left[\begin{array}{l}
\left.2 \pi r_{j} k_{m, i j} \frac{\partial}{\partial r} \bar{T}_{m, i j}^{r}(r, t)\right|_{r=r_{j}} \\
-\left.2 \pi r_{j-1} k_{m, i j} \frac{\partial}{\partial r} \bar{T}_{m, i j}^{r}(r, t)\right|_{r=r_{j-1}}
\end{array}\right] \Delta z_{i}+\bar{q}_{m, i j}^{\prime \prime \prime} V_{m, i j} .
\end{gathered}
$$

where $V_{m, i j}=\pi\left(r_{j}^{2}-r_{j-1}^{2}\right) \Delta z_{i}, A_{m, j}=\pi\left(r_{j}^{2}-r_{j-1}^{2}\right)$ and

$$
\bar{T}_{m, i j}=\frac{1}{V_{m, i j}} \int_{z_{i-1}}^{z_{i}} \int_{r_{j-1}}^{r_{j}} T_{m}(r, z, t) 2 \pi r d r d z
$$

Assuming that the temperature varies linearly from the center of the mesh cell to the midpoints of its surfaces, the derivatives at $r=r_{i}$ of two adjacent cells $(i, j)$ and $(i+1, j)$ can be obtained as

$$
\begin{gathered}
\left.\frac{\partial}{\partial r} \bar{T}_{m, i j}^{r}\right|_{r=r_{j}} \cong \frac{\bar{T}_{m, i, j+1 / 2}-\bar{T}_{m, i j}}{\Delta r_{j} / 2}, \\
\left.\frac{\partial}{\partial r} \bar{T}_{m, i, j+1}^{r}\right|_{r=r_{j}} \cong \frac{\bar{T}_{m, i, j+1}-\bar{T}_{m, i, j+1 / 2}}{\Delta r_{j+1} / 2} .
\end{gathered}
$$

where $\bar{T}_{i, j+1 / 2}$ is the surface average temperature of the interface of the cells $(i, j)$ and $(i, j+1)$. Thus the continuity conditions at the cell surface can be written as 


$$
-k_{m, i j} \frac{\bar{T}_{m, i, j+1 / 2}-\bar{T}_{m, i j}}{\Delta r_{j} / 2}=-k_{m, i, j+1} \frac{\bar{T}_{m, i, j+1}-\bar{T}_{m, i, j+1 / 2}}{\Delta r_{j+1} / 2},
$$

Eq. (C.34) can be solved for the interface temperature $\bar{T}_{i, j+1 / 2}$ as

$$
\bar{T}_{m, i, j+1 / 2}=\frac{\left(k_{m, i j} / \Delta r_{j}\right) \bar{T}_{m, i j}+\left(k_{m, i, j+1} / \Delta r_{j+1}\right) \bar{T}_{m, i, j+1}}{k_{m, i j} / \Delta r_{j}+k_{m, i, j+1} / \Delta r_{j+1}} .
$$

Then the heat flux at the interface can be given as

$$
\begin{gathered}
\bar{q}_{m, r, i}^{\prime \prime}(r, t)=-k_{m, i j} \frac{\partial}{\partial r}\left[\frac{1}{\Delta z_{i}} \int_{z_{i-1}}^{z_{i}} T_{m}(r, z, t) d z\right]=-k_{m, i j} \frac{\partial}{\partial r} \bar{T}_{i j}^{r}(r, t), \\
\left.\bar{q}_{m, r, i}^{\prime \prime}\right|_{r=r_{j}}=a_{j, j+1}^{r}\left(\bar{T}_{m, i j}-\bar{T}_{m, i, j+1}\right)=-a_{j+1, j}^{r}\left(\bar{T}_{m, i, j+1}-\bar{T}_{m, i j}\right),
\end{gathered}
$$

where

$$
a_{j, j+1}^{r}=\frac{1}{\Delta r_{j} /\left(2 k_{m, j}\right)+\Delta r_{j+1} /\left(2 k_{m, j+1}\right)} .
$$

When a cell surface corresponds to an external boundary, the external boundary condition in Eq. (C.24) provides the relation needed for determining the boundary coupling coefficients. Under the assumption that the temperature varies linearly from the center of the mesh cell to the midpoints of its surfaces, the temperature at a boundary can be approximated as

$$
\overline{T_{b}} \cong \bar{T}_{i j}+(\vec{n} \cdot \nabla T)\left(\Delta_{n} / 2\right)
$$

where $\Delta_{n}=\Delta r_{i}$ or $\Delta_{n}=\Delta z_{j}$. By substituting Eq. (C.39) into Eq. (C24), the normal derivative on a boundary surface can be obtained as

$$
\vec{n} \cdot \nabla T=-\frac{\beta}{\alpha k+\beta \Delta_{n} / 2} \bar{T}_{m, i j}+\frac{\gamma}{\alpha k+\beta \Delta_{n} / 2},
$$

Thus, the heat flux at the radial boundaries can be obtained as 


$$
\left.\bar{q}_{m, r, i}^{\prime \prime}\right|_{r=r_{b}}=-\left.k_{m, i j} \frac{\partial T}{\partial r}\right|_{r=r_{b}}=\left\{\begin{array}{cl}
a_{j, b}^{r} \bar{T}_{m, i j}-b_{j, b}^{r}, & \text { right boundary } \\
-a_{j, b}^{r} \bar{T}_{m, i j}+b_{j, b}^{r}, & \text { left boundary }
\end{array}\right.
$$

where

$$
\begin{aligned}
& a_{j b}^{r}=\frac{\beta}{\alpha+\beta \Delta r_{j} /\left(2 k_{m, i j}\right)}, \\
& b_{j b}^{r}=\frac{\gamma}{\alpha+\beta \Delta r_{j} /\left(2 k_{m, i j}\right)} .
\end{aligned}
$$

By inserting the surface fluxes in Eq. (C.37) and Eq. (C.41) into equation (C.30), the mesh-centered finite difference equations are obtained as

$$
\begin{gathered}
\frac{d}{d t}\left(\rho_{m, i j} c_{m, p, i j} \bar{T}_{m, i j}\right)=c_{i j, j+1} \bar{T}_{m, i, j+1}+c_{i j, j-1} \bar{T}_{m, i, j-1}-d_{i j} \bar{T}_{m, i j}+\bar{q}_{m, i j}^{\prime \prime \prime} \quad(2 \leq j \leq J-1), \\
\frac{d}{d t}\left(\rho_{i j} c_{p, i j} \bar{T}_{m, i j}\right)=c_{i j, j+1} \bar{T}_{m, i, j+1}-d_{i j} \bar{T}_{m, i j}+c_{j b} \bar{T}_{f j}+\bar{q}_{m, i j}^{\prime \prime \prime} \quad(j=1), \\
\frac{d}{d t}\left(\rho_{m, i j} c_{m, p, i j} \bar{T}_{m, i j}\right)=c_{i j, j-1} \bar{T}_{m, i, j-1}-d_{i j} \bar{T}_{m, i j}+\bar{q}_{m, i j}^{\prime \prime \prime} \quad(j=J) .
\end{gathered}
$$

where

$$
\begin{gathered}
C_{i j, j+1}=\frac{2 \pi r_{j} \Delta z_{i}}{V_{m, i j}} a_{j, j+1}^{r}=\frac{2 r_{j}}{r_{j}^{2}-r_{j-1}^{2}} a_{j, j+1}^{r}, \quad 1 \leq j \leq J-1 \\
C_{i j, j-1}=\frac{2 \pi r_{j-1} \Delta z_{i}}{V_{m, i j}} a_{j, j-1}^{r}=\frac{2 r_{j-1}}{r_{j}^{2}-r_{j-1}^{2}} a_{j, j-1}^{r}, \quad 2 \leq j \leq J \\
c_{j b}=\frac{2 \pi r_{0} \Delta z_{i}}{V_{m, i j}} a_{j b}^{r}=\frac{2 r_{0}}{r_{1}^{2}-r_{0}^{2}} \frac{h_{f i}}{1+h_{i j} \Delta r_{1} /\left(2 k_{m, i j}\right)}, \quad j=1 \\
d_{i j}= \begin{cases}c_{i j, j+1}+c_{i j, j-1}, & 2 \leq j \leq J-1 \\
c_{i j, j+1}+c_{j b}, & j=1 \\
c_{i j, j-1}, & j=J\end{cases}
\end{gathered}
$$




\section{C.3. Temporal Discretization}

The spatially discretized equations, Eq. (C.25), Eq. (C.26), and Eq. (C.27) provide a system of nonlinear ordinary differential equations for the cell-averaged temperatures, the cell-averaged pressures, and the cell-face velocities of the fuel salt. A system of linear equations can be obtained by employing a fully explicit temporal difference scheme. However, for incompressible flow, the fully explicit scheme cannot produce an equation to update the fuel salt pressure as shown below. On the other hand, the fully implicit scheme yields a system of nonlinear equations, which requires a more complicated solution scheme. As a compromise, a semi-implicit scheme can be used as in the RELAP-5 code [31]. This semi-implicit numerical solution scheme is based on replacing the system of differential equations with a system of finite difference equations partially implicit in time. The implicit terms are formulated to be linear in the dependent variables at a new time. This results in a linear time-advancement matrix that is solved by direct inversion using a sparse matrix routine. Implicitness is selected such that the field equations can be reduced to a single difference equation per fluid control volume or mesh cell, which is generally in terms of the hydrodynamic pressure [32].

\section{C.3.1. Fully Explicit Scheme}

Using the fully explicit temporal difference scheme, the finite difference equations for the mass, energy and momentum equations Eq. (C.25), Eq. (C.26), and Eq. (C.27) can be written as

$$
\begin{gathered}
\bar{\rho}_{i}^{n+1}=\bar{\rho}_{i}^{n}+\left(\bar{\rho}_{i-1}^{n} u_{i-1}^{n}-\bar{\rho}_{i}^{n} u_{i}^{n}\right) \frac{\Delta t_{n}}{\Delta z_{i}}, \\
\bar{T}_{i}^{n+1}=\bar{T}_{i}^{n}+\left[\frac{\bar{\rho}_{i-1}^{n}}{\bar{\rho}_{i}^{n}} u_{i-1}^{n}\left(\bar{T}_{i-1}^{n}-\bar{T}_{i}^{n}\right)+\frac{\bar{q}_{i}^{n}}{\bar{\rho}_{i}^{n} c_{p, i}^{n} A_{i}}+\frac{q_{i}^{\prime \prime n} P_{h, i} \Delta z_{i}}{\bar{\rho}_{i}^{n} c_{p, i}^{n} A_{i}}\right] \frac{\Delta t_{n}}{\Delta z_{i}}, \\
u_{i}^{n+1}=u_{i}^{n}+\left[\frac{\bar{\rho}_{i-1}^{n} u_{i-1}^{n}}{\bar{\rho}_{i}^{n}}\left(\frac{2}{\Delta z_{i}+\Delta z_{i+1}} \frac{\bar{\rho}_{i-1}^{n}}{\bar{\rho}_{i}^{n}} u_{i-1}^{n}-\frac{u_{i}^{n}}{\Delta z_{i}}\right)-\left(u_{i}^{n}\right)^{2}\left(\frac{2}{\Delta z_{i}+\Delta z_{i+1}} \frac{\bar{\rho}_{i}^{n}}{\bar{\rho}_{i+1}^{n}}-\frac{1}{\Delta z_{i}}\right)\right. \\
\left.+\frac{2}{\bar{\rho}_{i}^{n}\left(\Delta z_{i}+\Delta z_{i+1}\right)}\left(\bar{P}_{i}^{n}-\bar{P}_{i+1}^{n}\right)-\frac{\left(1+\bar{\rho}_{i+1}^{n} / \bar{\rho}_{i}^{n}\right)}{1+\Delta z_{i+1} / \Delta z_{i}} g-\frac{f_{i}\left(u_{i}^{n}\right)^{2}}{2 D_{h}}\right] \Delta t_{n} .
\end{gathered}
$$

Since the density is generally a function of temperature and pressure, Eq. (C.51) to Eq. (C.53) represent a system of equations for the pressure, temperature, and velocity at the new time node. Once the density, enthalpy, and velocity values at the new time node are 
determined, the new time pressure can be determined to yield the new time density. If the density is independent of pressure (i.e., incompressible), Eq. (C.51) and Eq. (C.52) are redundant, and there is no equation to update the pressure. Thus, this fully explicit scheme does not yield a proper system of equations.

With the fully explicit temporal difference scheme, the finite difference equations for the heat conduction equations in Eq. (C.44) through Eq. (C.46) can be obtained as

$$
\begin{gathered}
\bar{T}_{m, i j}^{n+1}=\left(1-\frac{d_{i j}^{n} \Delta t_{n}}{\rho_{m, i j}^{n} c_{p, m, i j}^{n}}\right) \bar{T}_{m, i j}^{n}+\frac{\Delta t_{n}}{\rho_{m, i j}^{n} c_{p, m, i j}^{n}}\left(c_{i j, j-1}^{n} \bar{T}_{m, i, j-1}^{n}+c_{i j, j+1}^{n} \bar{T}_{m, i, j+1}^{n}+\bar{q}_{m, i j}^{\prime \prime \prime}\right),(2 \leq j \leq J-1), \\
\bar{T}_{m, i j}^{n+1}=\left(1-\frac{d_{i j}^{n} \Delta t_{n}}{\rho_{m, i j}^{n} c_{p, m, i j}^{n}}\right) \bar{T}_{m, i j}^{n}+\frac{\Delta t_{n}}{\rho_{m, i j}^{n} c_{p, m, i j}^{n}}\left(c_{i j, j+1}^{n} \bar{T}_{m, i, j+1}^{n}+c_{j b}^{n} \bar{T}_{c i}^{n}+\bar{q}_{m, i j}^{\prime \prime \prime}\right), \quad(j=1), \\
\bar{T}_{m, i j}^{n+1}=\left(1-\frac{d_{i j}^{n} \Delta t_{n}}{\rho_{m, i j}^{n} c_{p, m, i j}^{n}}\right) \bar{T}_{m, i j}^{n}+\frac{\Delta t_{n}}{\rho_{m, i j}^{n} c_{p, m, i j}^{n}}\left(c_{i j, j-1}^{n} \bar{T}_{m, i, j-1}^{n}+\bar{q}_{m, i j}^{\prime \prime \prime}\right), \quad(j=J) .
\end{gathered}
$$

\section{C.3.2. Semi-Implicit Scheme}

Using the semi-implicit method of RELAP-5 [31] the finite difference equations for the mass, energy, and momentum equations in Eq. (C.25), Eq. (C.26), and Eq. (C.27) can be written as

$$
\begin{gathered}
\bar{\rho}_{i}^{n+1}=\bar{\rho}_{i}^{n}+\left(\bar{\rho}_{i-1}^{n} u_{i-1}^{n+1}-\bar{\rho}_{i}^{n} u_{i}^{n+1}\right) \frac{\Delta t_{n}}{\Delta z_{i}}, \\
\bar{T}_{i}^{n+1}=\bar{T}_{i}^{n}+\left[\frac{\bar{\rho}_{i-1}^{n}}{\bar{\rho}_{i}^{n}} u_{i-1}^{n+1}\left(\bar{T}_{i-1}^{n}-\bar{T}_{i}^{n}\right)+\frac{\bar{q}_{i}^{n}}{\bar{\rho}_{i}^{n} c_{p, i}^{n} A_{i}}+\frac{q_{i}^{\prime \prime n} P_{h, i} \Delta z_{i}}{\bar{\rho}_{i}^{n} c_{p, i}^{n} A_{i}}\right] \frac{\Delta t_{n}}{\Delta z_{i}}, \\
u_{i}^{n+1}=u_{i}^{n}+\left[\frac{\bar{\rho}_{i-1}^{n} u_{i-1}^{n}}{\bar{\rho}_{i}^{n}}\left(\frac{2}{\Delta z_{i}+\Delta z_{i+1}} \frac{\bar{\rho}_{i-1}^{n}}{\bar{\rho}_{i}^{n}} u_{i-1}^{n}-\frac{u_{i}^{n}}{\Delta z_{i}}\right)-\left(u_{i}^{n}\right)^{2}\left(\frac{2}{\Delta z_{i}+\Delta z_{i+1}} \frac{\bar{\rho}_{i}^{n}}{\bar{\rho}_{i+1}^{n}}-\frac{1}{\Delta z_{i}}\right)\right. \\
\left.+\frac{2}{\bar{\rho}_{i}^{n}\left(\Delta z_{i}+\Delta z_{i+1}\right)}\left(\bar{P}_{i}^{n+1}-\bar{P}_{i+1}^{n+1}\right)-\frac{\left(1+\bar{\rho}_{i+1}^{n} / \bar{\rho}_{i}^{n}\right)}{1+\Delta z_{i+1} / \Delta z_{i}} g-\frac{f_{i}\left(u_{i}^{n}\right)^{2}}{2 D_{h}}\right] \Delta t_{n} .
\end{gathered}
$$

For the node at the top, Eq. (C.59) becomes

$$
u_{I}^{n+1}=u_{I}^{n}+\left[\frac{\bar{\rho}_{I-1}^{n} u_{I-1}^{n}}{\bar{\rho}_{I}^{n} \Delta z_{I}}\left(2 \frac{\bar{\rho}_{I-1}^{n}}{\bar{\rho}_{I}^{n}} u_{I-1}^{n}-u_{I}^{n}\right)-\frac{\left(u_{I}^{n}\right)^{2}}{\Delta z_{I}}+\frac{2}{\bar{\rho}_{I}^{n} \Delta z_{I}}\left(\bar{P}_{I}^{n+1}-P_{\text {out }}^{n+1}\right)-g-\frac{f_{I}\left(u_{I}^{n}\right)^{2}}{2 D_{h}}\right] \Delta t_{n} \text {. }
$$


The provisional advanced time density can be obtained by linearizing the density state relation about the old-time value as

$$
\bar{\rho}_{i}^{n+1}=\bar{\rho}_{i}^{n}+\left.\frac{\partial \rho}{\partial T}\right|_{i} ^{n}\left(\bar{T}_{i}^{n+1}-\bar{T}_{i}^{n}\right)+\left.\frac{\partial \rho}{\partial P}\right|_{i} ^{n}\left(\bar{P}_{i}^{n+1}-\bar{P}_{i}^{n}\right) .
$$

Inserting Eq. (C.61) into the mass balance equation Eq. (C.57), then

$$
\left.\frac{\partial \rho}{\partial T}\right|_{i} ^{n}\left(\bar{T}_{i}^{n+1}-\bar{T}_{i}^{n}\right)+\left.\frac{\partial \rho}{\partial P}\right|_{i} ^{n}\left(\bar{P}_{i}^{n+1}-\bar{P}_{i}^{n}\right)=\left(\bar{\rho}_{i-1}^{n} u_{i-1}^{n+1}-\bar{\rho}_{i}^{n} u_{i}^{n+1}\right) \frac{\Delta t_{n}}{\Delta z_{i}} .
$$

Eliminating the new time temperature using Eq. (C.58) and Eq. (C.62), the provisional pressure change can be obtained as

$$
\begin{aligned}
\bar{P}_{i}^{n+1}-\bar{P}_{i}^{n} & =\left(\left.\frac{\partial \rho}{\partial P}\right|_{i} ^{n}\right)^{-1}\left\{\bar{\rho}_{i-1}^{n}\left[1-\left.\frac{1}{\bar{\rho}_{i}^{n}} \frac{\partial \rho}{\partial T}\right|_{i} ^{n}\left(\bar{T}_{i-1}^{n}-\bar{T}_{i}^{n}\right)\right] u_{i-1}^{n+1}-\bar{\rho}_{i}^{n} u_{i}^{n+1}\right\} \frac{\Delta t_{n}}{\Delta z_{i}} \\
& -\left.\left(\left.\frac{\partial \rho}{\partial P}\right|_{i} ^{n}\right)^{-1} \frac{\partial \rho}{\partial T}\right|_{i} ^{n}\left(\frac{\bar{q}_{i}^{n}}{\bar{\rho}_{i}^{n} c_{p, i}^{n} A_{i} \Delta z_{i}}+\frac{q_{i}^{\prime \prime n} P_{h, i}}{\bar{\rho}_{i}^{n} c_{p, i}^{n} A_{i}}\right) \Delta t_{n}
\end{aligned}
$$

Substituting the new time velocities in Eq. (C.59) into Eq. (C.63), a system of linear equations for $I$ cell-center pressures at new time can be obtained. Then, Eq. (C.59) and Eq. (C.63) can be written as

$$
\begin{gathered}
u_{i}^{n+1}=\alpha_{i}^{n}+\beta_{i}^{n}\left(\bar{P}_{i}^{n+1}-\bar{P}_{i+1}^{n+1}\right), \\
\bar{P}_{i}^{n+1}-\bar{P}_{i}^{n}=\xi_{i}^{n} u_{i-1}^{n+1}-\eta_{i}^{n} u_{i}^{n+1}-\zeta_{i}^{n},
\end{gathered}
$$

where $\bar{P}_{I+1}^{n+1}=P_{\text {out }}^{n+1}$ and

$$
\begin{aligned}
\alpha_{i}^{n}=u_{i}^{n}+ & {\left[\frac{\bar{\rho}_{i-1}^{n} u_{i-1}^{n}}{\bar{\rho}_{i}^{n}}\left(\frac{2}{\Delta z_{i}+\Delta z_{i+1}} \frac{\bar{\rho}_{i-1}^{n}}{\bar{\rho}_{i}^{n}} u_{i-1}^{n}-\frac{u_{i}^{n}}{\Delta z_{i}}\right)-\left(u_{i}^{n}\right)^{2}\left(\frac{2}{\Delta z_{i}+\Delta z_{i+1}} \frac{\bar{\rho}_{i}^{n}}{\bar{\rho}_{i+1}^{n}}-\frac{1}{\Delta z_{i}}\right)\right.} \\
& \left.-\frac{\left(1+\bar{\rho}_{i+1}^{n} / \bar{\rho}_{i}^{n}\right)}{1+\Delta z_{i+1} / \Delta z_{i}} g-\frac{f_{i}\left(u_{i}^{n}\right)^{2}}{2 D_{h}}\right] \Delta t_{n}, \quad i=1,2, \cdots, I-1, \\
\alpha_{I}^{n}= & u_{I}^{n}+\left[\frac{\bar{\rho}_{I-1}^{n} u_{I-1}^{n}}{\bar{\rho}_{I}^{n} \Delta z_{I}}\left(2 \frac{\bar{\rho}_{I-1}^{n}}{\bar{\rho}_{I}^{n}} u_{I-1}^{n}-u_{I}^{n}\right)-\frac{\left(u_{I}^{n}\right)^{2}}{\Delta z_{I}}-g-\frac{f_{I}\left(u_{I}^{n}\right)^{2}}{2 D_{h}}\right] \Delta t_{n},
\end{aligned}
$$




$$
\begin{gathered}
\beta_{i}^{n}=\frac{2 \Delta t_{n}}{\bar{\rho}_{i}^{n}\left(\Delta z_{i}+\Delta z_{i+1}\right)}, \quad i=1,2, \cdots, I-1, \\
\beta_{I}^{n}=\frac{2 \Delta t_{n}}{\bar{\rho}_{I} \Delta z_{I}}, \\
\xi_{i}^{n}=\bar{\rho}_{i-1}^{n}\left(\left.\frac{\partial \rho}{\partial P}\right|_{i} ^{n}\right)^{-1}\left[1-\left.\frac{1}{\bar{\rho}_{i}^{n}} \frac{\partial \rho}{\partial T}\right|_{i} ^{n}\left(\bar{T}_{i-1}^{n}-\bar{T}_{i}^{n}\right)\right] \frac{\Delta t_{n}}{\Delta z_{i}}, \\
\zeta_{i}^{n}=\left.\left(\left.\frac{\partial \rho}{\partial P}\right|_{i} ^{n}\right)^{-1} \frac{\partial \rho}{\partial T}\right|_{i} ^{n}\left(\frac{\bar{q}_{i}^{n}}{\eta_{i}^{n} c_{p, i}^{n} A_{i} \Delta z_{i}}+\frac{q_{i}^{\prime \prime n} P_{h, i}}{\bar{\rho}_{i}^{n} c_{p, i}^{n} A_{i}}\right) \Delta t_{n} .
\end{gathered}
$$

Substituting the new time velocities $u_{i}^{n+1}$ and $u_{i-1}^{n+1}$ for $i=2,3, \cdots, I-1$ in Eq. (C.64) into Eq. (C.65), will result in

$$
\begin{array}{r}
\xi_{i}^{n} \beta_{i-1}^{n} \bar{P}_{i-1}^{n+1}-\left(1+\xi_{i}^{n} \beta_{i-1}^{n}+\eta_{i}^{n} \beta_{i}^{n}\right) \bar{P}_{i}^{n+1}+\eta_{i}^{n} \beta_{i}^{n} \bar{P}_{i+1}^{n+1}=\zeta_{i}^{n}-\xi_{i}^{n} \alpha_{i-1}^{n}+\eta_{i}^{n} \alpha_{i}^{n}-\bar{P}_{i}^{n}, \\
i=2,3, \cdots, I-1,
\end{array}
$$

Using the specified outlet pressure boundary condition, i.e., $\bar{P}_{I+1}^{n+1}=P_{\text {out }}^{n+1}$, the pressure equation for $\boldsymbol{i}=\boldsymbol{I}$ can be obtained as

$$
\xi_{i}^{n} \beta_{i-1}^{n} \bar{P}_{i-1}^{n+1}-\left(1+\xi_{i}^{n} \beta_{i-1}^{n}+\eta_{i}^{n} \beta_{i}^{n}\right) \bar{P}_{i}^{n+1}=\zeta_{i}^{n}-\xi_{i}^{n} \alpha_{i-1}^{n}+\eta_{i}^{n} \alpha_{i}^{n}-\bar{P}_{i}^{n}-\eta_{i}^{n} \beta_{i}^{n} \bar{P}_{\text {out }}^{n+1}, \quad i=I,
$$

If the inlet velocity $u_{i n}$ is given, the pressure equation for $i=1$ can be derived by inserting $u_{1}^{n+1}$ in Eq. (C.64) into Eq. (C.65) as

$$
-\left(1+\eta_{i}^{n} \beta_{i}^{n}\right) \bar{P}_{i}^{n+1}+\eta_{i}^{n} \beta_{i}^{n} \bar{P}_{i+1}^{n+1}=\zeta_{i}^{n}-\xi_{i}^{n} u_{i n}^{n+1}+\eta_{i}^{n} \alpha_{i}^{n}-\bar{P}_{i}^{n}, \quad i=1 .
$$

For specified inlet flow rate and temperature, Eq. (C.58) and Eq. (C.62) for $i=1$ can be written as

$$
\bar{T}_{i}^{n+1}=\bar{T}_{i}^{n}+\left[\frac{\rho_{i n}^{n+1} u_{i n}^{n+1}}{\bar{\rho}_{i}^{n}}\left(\bar{T}_{i n}^{n}-\bar{T}_{i}^{n}\right)+\frac{\bar{q}_{i}^{n}}{\bar{\rho}_{i}^{n} c_{p, i}^{n} A_{i}}+\frac{q_{i}^{\prime \prime n} P_{h, i} \Delta z_{i}}{\bar{\rho}_{i}^{n} c_{p, i}^{n} A_{i}}\right] \frac{\Delta t_{n}}{\Delta z_{i}},
$$




$$
\left.\frac{\partial \rho}{\partial T}\right|_{i} ^{n}\left(\bar{T}_{i}^{n+1}-\bar{T}_{i}^{n}\right)+\left.\frac{\partial \rho}{\partial P}\right|_{i} ^{n}\left(\bar{P}_{i}^{n+1}-\bar{P}_{i}^{n}\right)=\frac{1}{\Delta z_{i}}\left(\rho_{i n}^{n+1} u_{i n}^{n+1}-\bar{\rho}_{i}^{n} u_{i}^{n+1}\right) \Delta t_{n},
$$

As a result, Eq. (C.65) can be written as

$$
\bar{P}_{i}^{n+1}-\bar{P}_{i}^{n}=\tilde{\xi}_{i}^{n} \rho_{i n}^{n+1} u_{i n}^{n+1}-\eta_{i}^{n} u_{i}^{n+1}-\zeta_{i}^{n},
$$

where

$$
\begin{gathered}
\tilde{\xi}_{i}^{n}=\left(\left.\frac{\partial \rho}{\partial P}\right|_{i} ^{n}\right)^{-1}\left[1-\left.\frac{1}{\bar{\rho}_{i}^{n}} \frac{\partial \rho}{\partial T}\right|_{i} ^{n}\left(\bar{T}_{i n}^{n}-\bar{T}_{i}^{n}\right)\right] \frac{\Delta t_{n}}{\Delta z_{i}}, \\
\xi_{i}^{n}=\bar{\rho}_{i-1}^{n}\left(\left.\frac{\partial \rho}{\partial P}\right|_{i} ^{n}\right)^{-1}\left[1-\left.\frac{1}{\bar{\rho}_{i}^{n}} \frac{\partial \rho}{\partial T}\right|_{i} ^{n}\left(\bar{T}_{i-1}^{n}-\bar{T}_{i}^{n}\right)\right] \frac{\Delta t_{n}}{\Delta z_{i}}, \\
\eta_{i}^{n}=\left(\left.\frac{\partial \rho}{\partial P}\right|_{i} ^{n}\right)^{-1} \frac{\bar{\rho}_{i}^{n}}{\Delta z_{i}} \Delta t_{n}, \\
\zeta_{i}^{n}=\left.\left(\left.\frac{\partial \rho}{\partial P}\right|_{i} ^{n}\right)^{-1} \frac{\partial \rho}{\partial T}\right|_{i} ^{n}\left(\frac{\bar{q}_{i}^{n}}{\bar{\rho}_{i}^{n} c_{p, i}^{n} A_{i} \Delta z_{i}}+\frac{q_{i}^{\prime \prime n} P_{h, i}}{\bar{\rho}_{i}^{n} c_{p, i}^{n} A_{i}}\right) \Delta t_{n} .
\end{gathered}
$$

Inserting $u_{1}^{n+1}$ in Eq. (C.65) into Eq. (C.79), the pressure equation for $i=1$ can be derived as

$$
-\left(1+\eta_{i}^{n} \beta_{i}^{n}\right) \bar{P}_{i}^{n+1}+\eta_{i}^{n} \beta_{i}^{n} \bar{P}_{i+1}^{n+1}=\zeta_{i}^{n}-\tilde{\xi}_{i}^{n} w_{i n}^{n+1}-\bar{P}_{i}^{n}, \quad i=1 .
$$

The coefficient matrix for the system of linear equations for the time advanced pressures given in Eq. (C.73), Eq. (C.74), and Eq. (C.75) is a tri-diagonal matrix. Thus, this system of equations can easily be solved by the forward elimination and backward substitution algorithm. Substitution of the new time pressures into Eq. (C.65) yields the new time velocities. Using the new time velocities, the new time densities and temperatures can be obtained from Eq. (C.57) and Eq. (C.58), respectively. For an incompressible flow, the density does not depend on the pressure. In this case, the provisional advanced time density in Eq. (C.61) can be linearized as

$$
\bar{\rho}_{i}^{n+1}=\bar{\rho}_{i}^{n}+\left.\frac{\partial \rho}{\partial T}\right|_{i} ^{n}\left(\bar{T}_{i}^{n+1}-\bar{T}_{i}^{n}\right)
$$

Inserting Eq. (C.84) into the mass balance equation Eq. (C.58), then 


$$
\left.\frac{\partial \rho}{\partial T}\right|_{i} ^{n}\left(\bar{T}_{i}^{n+1}-\bar{T}_{i}^{n}\right)=\left(\bar{\rho}_{i-1}^{n} u_{i-1}^{n+1}-\bar{\rho}_{i}^{n} u_{i}^{n+1}\right) \frac{\Delta t_{n}}{\Delta z_{i}} .
$$

Eliminating the new time temperature using Eq. (C.85) and Eq. (C.58), the cell-face velocities at new time can be obtained as

$$
u_{i}^{n+1}=\omega_{i}^{n} u_{i-1}^{n+1}-\chi_{i}^{n},
$$

where

$$
\begin{gathered}
\omega_{i}^{n}=\frac{\bar{\rho}_{i-1}^{n}}{\bar{\rho}_{i}^{n}}\left[1-\left.\frac{1}{\bar{\rho}_{i}^{n}} \frac{\partial \rho}{\partial T}\right|_{i} ^{n}\left(\bar{T}_{i-1}^{n}-\bar{T}_{i}^{n}\right)\right], \\
\chi_{i}^{n}=\left.\frac{1}{\bar{\rho}_{i}^{n}} \frac{\partial \rho}{\partial T}\right|_{i} ^{n}\left(\frac{\bar{q}_{i}^{n}}{\bar{\rho}_{i}^{n} c_{p, i}^{n} A_{i}}+\frac{q_{i}^{\prime \prime} P_{h, i} \Delta z_{i}}{\bar{\rho}_{i}^{n} c_{p, i}^{n} A_{i}}\right) .
\end{gathered}
$$

Substituting the new time velocities $u_{i}^{n+1}$ and $u_{i-1}^{n+1}$ for $i=2,3, \cdots, I-1$ in Eq. (C.65) into Eq. (C.86), will result in

$$
\omega_{i}^{n} \beta_{i-1}^{n} \bar{P}_{i-1}^{n+1}-\left(\omega_{i}^{n} \beta_{i-1}^{n}+\beta_{i}^{n}\right) \bar{P}_{i}^{n+1}+\beta_{i}^{n} \bar{P}_{i+1}^{n+1}=\chi_{i}^{n}-\omega_{i}^{n} \alpha_{i-1}^{n}+\alpha_{i}^{n}, \quad i=2,3, \cdots, I,
$$

For the outlet pressure boundary condition, $\bar{P}_{I+1}^{n+1}=P_{\text {out }}^{n+1}$, the pressure equation for $i=I$ can be obtained as

$$
\omega_{i}^{n} \beta_{i-1}^{n} \bar{P}_{i-1}^{n+1}-\left(\omega_{i}^{n} \beta_{i-1}^{n}+\beta_{i}^{n}\right) \bar{P}_{i}^{n+1}=\chi_{i}^{n}-\omega_{i}^{n} \alpha_{i-1}^{n}+\alpha_{i}^{n}-\beta_{i}^{n} P_{\text {out }}^{n+1}, \quad i=I,
$$

Since the density is a function of temperature only, the inlet velocity can be determined for specified inlet flow rate and temperature. Therefore, using a known inlet velocity, the pressure equation for $i=1$ can be derived by inserting $u_{1}^{n+1}$ in Eq. (C.65) into Eq. (C.77) as

$$
-\beta_{i}^{n} \bar{P}_{i}^{n+1}+\beta_{i}^{n} \bar{P}_{i+1}^{n+1}=\chi_{i}^{n}-\omega_{i}^{n} u_{i n}^{n+1}+\alpha_{i}^{n}, \quad i=1
$$

The coefficient matrix for the system of linear equations for the time advanced pressures given in Eq. (C.89), Eq. (C.90), and Eq. (C.91), is a tri-diagonal matrix. Thus, this system of equations can easily be solved by the forward elimination and backward substitution algorithm. Substitution of the new time pressures into Eq. (C.59) yields the new time velocities. Using the new time velocities, the new time densities and temperatures can be obtained from Eq. (C.57) and Eq. (C.58), respectively. 
Using the fully implicit temporal difference scheme, the difference equations for the heat conduction equations in Eq. (C.44) to Eq. (C.46) can be obtained as

$$
\begin{gathered}
-\frac{c_{i j, j-1}^{n+1} \Delta t_{n}}{\rho_{m, i j}^{n+1} c_{p, m, i j}^{n+1}} \bar{T}_{m, i, j-1}^{n+1}+\left(1+\frac{d_{i j}^{n+1} \Delta t_{n}}{\rho_{m, i j}^{n+1} c_{p, m, i j}^{n+1}}\right) \bar{T}_{m, i, j}^{n+1}-\frac{c_{i j, j+1}^{n+1} \Delta t_{n}}{\rho_{m, i j}^{n+1} c_{p, m, i j}^{n+1}} \bar{T}_{m, i, j+1}^{n+1}=\bar{T}_{m, i, j}^{n}+\frac{\Delta t_{n}}{\rho_{m, i j}^{n+1} c_{p, m, i j}^{n+1}} \bar{q}_{m, i j}^{\prime \prime \prime+1}, \\
(2 \leq j \leq J-1), \\
\left(1+\frac{d_{i j}^{n+1} \Delta t_{n}}{\rho_{m, i j}^{n+1} c_{p, m, i j}^{n+1}}\right) \bar{T}_{m, i, j}^{n+1}-\frac{c_{i j, j+1}^{n+1} \Delta t_{n}}{\rho_{m, i j}^{n+1} c_{p, m, i j}^{n+1}} \bar{T}_{m, i, j+1}^{n+1}=\bar{T}_{m, i, j}^{n}+\frac{\Delta t_{n}}{\rho_{m, i j}^{n+1} c_{p, m, i j}^{n+1}}\left(\bar{q}_{m, i j}^{m+n+1}+c_{j b}^{n+1} \bar{T}_{c i}^{n+1}\right), \quad(j=1), \\
-\frac{c_{i j, j-1}^{n+1} \Delta t_{n}}{\rho_{m, i j}^{n+1} c_{p, m, i j}^{n+1}} \bar{T}_{m, i, j-1}^{n+1}+\left(1+\frac{d_{i j}^{n+1} \Delta t_{n}}{\rho_{m, i j}^{n+1} c_{p, m, i j}^{n+1}}\right) \bar{T}_{m, i, j}^{n+1}=\bar{T}_{m, i, j}^{n}+\frac{\Delta t_{n}}{\rho_{m, i j}^{n+1} c_{p, m, i j}^{n+1}} \bar{q}_{m, i j}^{m+n+1}, \quad(j=J) .
\end{gathered}
$$

The finite difference equations in Eq. (C.92) through Eq. (C.94) form a system of nonlinear equations since the coefficients depend on the unknown temperatures through the temperature dependent material properties. This system of nonlinear equations can be solved iteratively. These coefficients can initially be estimated using an initial guess of temperature. For known coefficients, Eq. (C.92) through Eq. (C.94) form a system of linear equations, which is a tri-diagonal system. This tri-diagonal system can easily be solved by the forward elimination and backward substitution algorithm. Then, the coefficients are updated using the calculated temperatures. This iteration continues until the successive temperatures converge within a specified limit.

\section{C.4. Steady State Solution Methods}

The balance equations in Eq. (C.84), Eq. (C.58), and Eq. (C.86) couple the field and flow variables of three meshes $i-1, i$, and $i+1$, and hence they need to be solved simultaneously. This discretization scheme provides a stable time-dependent solution. By setting the time derivatives in Eq. (C.1), Eq. (C.2), and Eq. (C.3) to zero and by applying the finite difference discretization scheme, the steady state equations for an upward flow in a constant flow area can be derived as

$$
\begin{gathered}
\bar{T}_{i}=\left(1+\frac{P_{h, i} \Delta z_{i} h_{f, i}}{\bar{c}_{p, i} \bar{\rho}_{i-1} u_{i-1} A_{i}}\right)^{-1}\left(\bar{T}_{i-1}+\frac{\bar{q}_{i}}{\bar{c}_{p, i} \bar{\rho}_{i-1} u_{i-1} A_{i}}+\frac{P_{h, i} \Delta z_{i} h_{f, i}}{\bar{c}_{p, i} \bar{\rho}_{i-1} u_{i-1} A_{i}} \bar{T}_{m, i}\right), \\
\rho_{i}=\rho(T), \\
u_{i}=\frac{\bar{\rho}_{i-1}}{\bar{\rho}_{i}} u_{i-1},
\end{gathered}
$$




$$
\bar{P}_{i}=\bar{P}_{i+1}-\left[\left(\frac{\bar{\rho}_{i-1}^{2}}{\bar{\rho}_{i}} u_{i-1}^{2}-\frac{\bar{\rho}_{i}^{2}}{\bar{\rho}_{i+1}} u_{i}^{2}\right)-\frac{\left(\bar{\rho}_{i} \Delta z_{i}+\bar{\rho}_{i+1} \Delta z_{i+1}\right)}{2} g-\frac{f_{i} \bar{\rho}_{i} u_{i}^{2} \Delta z_{i}}{2 D_{h}}\right] .
$$

The steady state solution scheme is started by solving Eq. (C.95) for the temperature and then obtaining the density and velocity in each node by solving Eq. (C.96) and Eq. (C.97) respectively, until the end of the channel. The pressure in each node is solved using Eq. (C.98) starting from the end of the channel to the channel inlet, node by node.

The steady state heat condition in the moderator can be derived by setting the time derivative in Eq. (C.44) through Eq. (C.46) to zero and neglecting the axial conduction as

$$
\begin{gathered}
-c_{i j, j-1} \bar{T}_{i, j-1}+d_{i j} \bar{T}_{i, j}-c_{i j, j+1} \bar{T}_{i, j+1}=\bar{q}_{i j}^{\prime \prime \prime}, \quad(2 \leq j \leq J-1), \\
d_{i j} \bar{T}_{i, j}-c_{i j, j+1} \bar{T}_{i, j+1}=c_{j b} \bar{T}_{c i}+\bar{q}_{i j}^{\prime \prime \prime}, \quad(j=1), \\
-c_{i j, j-1} \bar{T}_{i, j-1}+d_{i j} \bar{T}_{i, j}=\bar{q}_{i j}^{\prime \prime \prime}, \quad(j=J) .
\end{gathered}
$$

The finite difference equations in Eq. (C.99) through Eq. (C.101) form a system of nonlinear equations since the coefficients depend on the unknown temperatures through the temperature dependent material properties. This system of equations forms a tri-diagonal system that can easily be solved by the forward elimination and backward substitution algorithm.

\section{C.5. Flow Splitting Algorithm}

It is assumed that the total fuel salt flow is distributed among multiple parallel fuel channels that have common inlet and outlet. The mass flows in individual fuel channels are calculated using the equal pressure drop boundary conditions over all channels. For given mass flux $G_{i}$, the pressure drop $\Delta P_{i}$ across the fuel channel $i$ is determined by solving the single channel thermal-hydraulics problem. Denoting the pressure drop as a function of the mass flux as $\Delta P_{i}\left(G_{i}\right)$, the system of governing equations for unknown mass fluxes for $I$ parallel fuel channels can be written as the following system of nonlinear equations

$$
\begin{gathered}
\Delta P_{1}\left(G_{1}\right)=\Delta P_{2}\left(G_{2}\right)=\ldots=\Delta P_{I}\left(G_{i}\right)=\overline{\Delta P}, \\
\sum_{i=1}^{I} N_{i} A_{i} G_{i}=W_{T} .
\end{gathered}
$$


where $N_{i}$ is the number of coolant tubes, $A_{i}$ is the flow area of channel $i$, and $W_{T}$ is the total flow rate. The system of nonlinear equations given in Eq. (C.102) and Eq. (C.103) can be solved iteratively using the Newton-Rapson method. The derivatives required in the NewtonRapson method can be estimated by the finite difference approximation based on the single channel solutions for given mass fluxes. Using the pressure drop $\Delta P_{i}^{(n)}$ calculated with the $n$ th iterative solution $G_{i}^{(n)}$, the pressure drop for the $(n+1)$-st solution can be approximated as

$$
\Delta P_{i}^{(n+1)}=\Delta P_{i}^{(n)}+a_{i}^{(n)}\left(G_{i}^{(n+1)}-G_{i}^{(n)}\right), \quad i=1,2, \ldots, I,
$$

where

$$
\begin{gathered}
a_{i}^{(n)}=\left.\frac{d\left(\Delta P_{i}\right)}{d\left(G_{i}\right)}\right|_{G_{i}=G_{i}^{(n)}}=\frac{\Delta P_{i}^{(n)}-\Delta P_{i}^{(n-1)}}{G_{i}^{(n)}-G_{i}^{(n-1)}}, \\
\Delta P_{i}^{(n)}=\Delta P_{i}\left(G_{i}^{(n)}\right) .
\end{gathered}
$$

Requiring $\Delta P_{i}^{(n+1)}$ to be equal to average pressure drop $\overline{\Delta P}^{(n+1)}$, Eq. (C.104) can be rewritten as

$$
a_{i}^{(n)} G_{i}^{(n+1)}-\overline{\Delta P}^{(n+1)}=a_{i}^{(n)} G_{i}^{(n)}-\Delta P_{i}^{(n)} \quad, \quad i=1,2, \ldots, I .
$$

Dividing Eq. (C.107) by $a_{i}^{(n)}$, multiplying by $N_{i} A_{i}$, and summing up the resulting equations, then

$$
\sum_{i=1}^{I} N_{i} A_{i} G_{i}^{(n+1)}-\sum_{i=1}^{I} \frac{N_{i} A_{i}}{a_{i}^{(n)}} \overline{\Delta P}^{(n+1)}=\sum_{i=1}^{I} N_{i} A_{i} G_{i}^{(n)}-\sum_{i=1}^{I} \frac{N_{i} A_{i} \Delta P_{i}^{(n+1)}}{a_{i}^{(n)}}
$$

Requiring the mass fluxes $G_{i}^{(n)}$ and $G_{i}^{(n+1)}$ to satisfy the flow conservation equation in Eq. (C.103), the average core pressure drop of the next iteration can be determined as

$$
\overline{\Delta P}^{(n+1)}=\sum_{i=1}^{I} \frac{N_{i} A_{i} \Delta P_{i}^{(n)}}{a_{i}^{(n)}} / \sum_{i=1}^{I} \frac{N_{i} A_{i}}{a_{i}^{(n)}},
$$

Using this average pressure drop, the mass fluxes of the next iteration are obtained as 


$$
G_{i}^{(n+1)}=G_{i}^{(n)}+\frac{1}{a_{i}^{(n)}}\left(\overline{\Delta P}^{(n+1)}-\Delta P_{i}^{(n)}\right), \quad i=1,2, \ldots, I .
$$

This iteration procedure continues until the mass fluxes of two subsequent iterations converge within a specified limit. Specifically, the channel flow rates are determined to satisfy the constant pressure drop boundary condition. Initial guess is made for each channel mass flux and the core-average pressure drop using the values at the previous time step. For the initial steady state, a uniform initial mass flux $G_{i}^{(0)}=W_{T} / \sum_{i=1}^{I} N_{i} A_{i}$ and the core average pressure drop $\left(\overline{\Delta P}^{(0)}\right)$ is used. Using the assumed mass flux $G_{i}^{(0)}$, the channel pressure drop $\Delta P_{i}^{(0)}$ is computed for each channel.

For a perturbed mass flux $G_{i}^{(1)}=\left(1+\varepsilon_{G}\right) G_{i}^{(0)}$, the perturbed channel pressure drop $\Delta P_{i}^{(1)}$ is computed. Here $\varepsilon_{G}$ is the user-specified parameter for the flow rate perturbation used to evaluate the pressure derivatives initially. Using these perturbed pressure drops, the pressure derivatives with respect to the mass flux and the core-average pressure drop are computed as

$$
\begin{gathered}
a_{i}^{(1)} \cong a_{i}^{(2)} \cong \frac{\Delta P_{i}^{(2)}-\Delta P_{i}^{(1)}}{\varepsilon_{G} G_{i}^{(1)}}, \\
\overline{\Delta P}^{(2)}=\sum_{i=1}^{I} \frac{A_{i} \Delta P_{i}^{(1)}}{a_{i}^{(1)}} / \sum_{i=1}^{I} \frac{A_{i}}{a_{i}^{(1)}} .
\end{gathered}
$$

The $(n+1)$-st iteration of each channel mass flux $G_{i}^{(n+1)}$ is determined to satisfy the constant pressure drop boundary conditions approximately as

$$
G_{i}^{(n+1)}=G_{i}^{(n)}+\frac{1}{a_{i}^{(n)}}\left(\overline{\Delta P}^{(n)}-\Delta P_{i}^{(n)}\right) .
$$

If the summation of individual channel flow rates is equal to the core flow rate, that is, $\left|\sum_{I=1}^{i} N_{i} A_{i} G_{i}^{(n+1)}-W_{T}\right| / W_{T}<\varepsilon_{w}$, then the constant pressure drop boundary condition is satisfied. The iteration for channel mass fluxes is stopped, and the single channel analysis is performed for each channel. Otherwise, the updated channel pressure drops $\Delta P_{i}^{(n+1)}$ are 
calculated with the channel flow rates $G_{i}^{(n+1)}$ and the pressure derivatives with respect to mass flux are updated as

$$
a_{i}^{(n+1)}=\frac{\Delta P_{i}^{(n+1)}-\Delta P_{i}^{(n)}}{G_{i}^{(n+1)}-G_{i}^{(n)}} .
$$

and back to Eq. (C.113) until it satisfies the convergence criteria.

\section{C.6. Primary Loop Thermal-Fluidic Model}

In the reactor system, the fuel salt exiting the core flows through the primary system before returning to the core inlet. To describe the core behavior during transients more accurately, the heat exchanger in the primary system is also included in the thermalhydraulics model of MSR. The heat exchanger has been modeled with a lumped parameter approach to simulate the heat removal by the intermediate loop, considering it as one node. As shown in Fig. C.4, the fuel salt temperatures at the core inlet and outlet are directly coupled with the fuel salt temperatures at the heat exchanger outlet and inlet, respectively. The other components of the primary loop such as primary pumps, inlet and outlet plenums are not considered in this model.

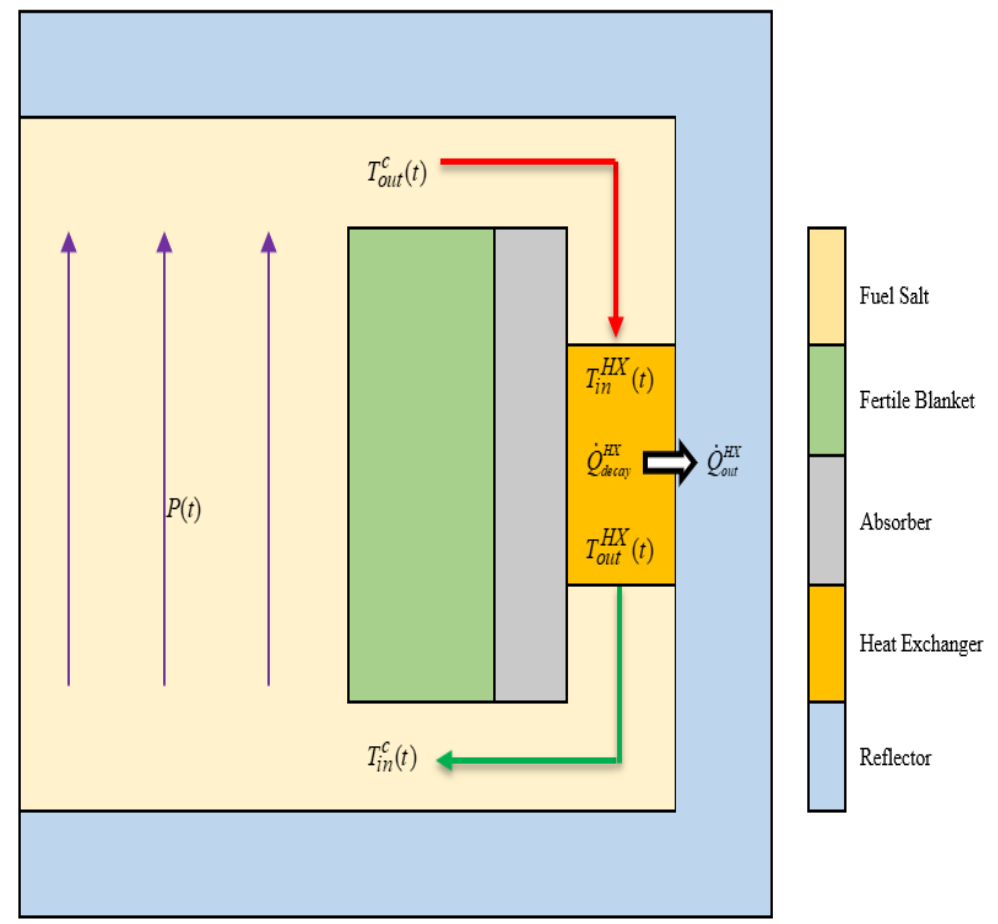

Fig. C.4. Schematic diagram of the heat removal from the primary system of MSFR. 
Assuming the material temperature variations in the heat exchanger during a transient are proportional to the steady state temperatures, the heat exchanger can be characterized by the average fuel salt temperature $\bar{T}_{H X}$, of which variation is determined by

$$
C_{H X} \frac{d \bar{T}_{H X}}{d t}=\dot{m}_{H X} c_{p}\left(T_{i n}^{H X}-T_{o u t}^{H X}\right)+\dot{Q}_{\text {decay }}-\dot{Q}_{H X} \cdot
$$

where $C_{H X}=m_{H X} c_{p}$ is the heat capacity of the heat exchanger, $c_{p}$ is the specific heat of the fuel salt at the corresponding temperature, $\dot{m}_{H X}$ is the fuel salt mass flow rate, and $m_{H X}$ is the mass of the heat exchanger. $T_{\text {in }}^{H X}$ and $T_{\text {out }}^{H X}$ are respectively the fuel salt temperatures at the inlet and outlet of the heat exchanger, $\dot{Q}_{\text {decay }}$ is the decay heat generated by the fuel salt in the heat exchanger, and $\dot{Q}_{H X}$ is the heat removal rate by the intermediate fluid in the heat exchanger, and it can be written as

$$
\dot{Q}_{H X}=U A\left(\bar{T}_{H X}-\bar{T}_{I F}\right) .
$$

where $\bar{T}_{I F}$ is the average intermediate fluid temperature, $U$ is the overall heat transfer coefficient from the fuel salt to the intermediate fluid, and $A$ is total heat transfer area in the heat exchanger.

The overall heat transfer coefficient $U$ is evaluated as

$$
\frac{1}{A U}=\left[\frac{1}{A h_{c}}+\frac{1}{A_{h} h_{h}}+R_{w}\right]^{-1},
$$

where $h_{c}$ is the heat transfer coefficient of the secondary side of the heat exchanger, $R_{w}$ is the thermal resistance of the heat exchanger wall, $A_{h}$ is the total transfer area of the hot fuel salt, and $h_{h}$ is the heat transfer coefficient of the fuel salt in the primary side of the heat exchanger. The primary side heat transfer coefficient is evaluated from the Gnielinski correlation for the Nusselt number [33] 


$$
\mathrm{Nu}=\frac{(f / 8)(\mathrm{Re}-1000) \operatorname{Pr}}{1+12.7(f / 8)^{0.5}\left(\operatorname{Pr}^{2 / 3}-1\right)}
$$

where $f$ is the friction factor in Eq. (C.5). For the secondary side heat transfer coefficient, it is assumed to be constant and it is evaluated from the steady state calculations. Using the upwind scheme for spatial differencing with $T_{\text {in }}^{H X}=T_{\text {out }}^{c}, T_{\text {out }}^{H X}=\bar{T}_{H X}$, and $T_{\text {in }}^{c}=T_{\text {out }}^{H X}$ and the explicit scheme for time differencing, the finite difference equation for heat exchanger can be derived as

$$
\bar{T}_{H X}^{n}=\bar{T}_{H X}^{n-1}+\frac{\dot{m}_{H X}^{n-1} C_{p}^{n-1}}{C_{H X}}\left(T_{\text {in }}^{n-1}-T_{\text {out }}^{n-1}\right) \Delta t_{n}-\frac{\Delta t_{n}}{C_{H X}}\left(\dot{Q}_{H X}^{n-1}-\dot{Q}_{\text {decay }}^{n-1}\right) .
$$

Eq. (C.119) can be rewritten with the initial condition as

$$
\begin{gathered}
\bar{T}_{H X}^{n}=\left[1-\frac{\dot{m}_{H X}^{n-1} c_{p}^{n-1} \Delta t_{n}}{C_{H X}}\right] \bar{T}_{H X}^{n-1}+\frac{\dot{m}_{H X}^{n-1} c_{p}^{n-1} \Delta t_{n}}{C_{H X}} T_{i n}^{n-1}-\frac{\Delta t_{n}}{C_{H X}}\left(\dot{Q}_{H X}^{n-1}-\dot{Q}_{\text {decay }}^{n-1}\right), \\
\bar{T}_{H X}^{0}=T_{\text {out }}^{c}-\frac{\dot{Q}_{H X}^{0}}{\dot{m}_{H X}^{0} c_{p}^{0}} .
\end{gathered}
$$

\section{C.7. Decay Heat Model}

The decay heat equation for a flowing fuel is different from that of a stationary fuel. In an MSR, the decay heat can be released outside the core, especially in the heat exchanger, and it should be considered in calculating the power. A simple decay heat model has been implemented into the PROTEUS-NODAL code, where the fission products are grouped into a few decay heat precursor groups $K_{D}$ and for each group, the following decay heat equation is solved

$$
\frac{\partial}{\partial t} h_{k}(\vec{r}, t)+\nabla \cdot\left[\vec{u}(\vec{r}, t) h_{k}(\vec{r}, t)\right]+\lambda_{k} h_{k}(\vec{r}, t)=f_{k} Q_{t o t}(\vec{r}, t), \quad k=1,2, \ldots ., K_{D}
$$

where $h_{k}$ is the product of the decay heat precursor concentration and energy release in decay heat group $k, f_{k}$ is the decay heat fraction in group $k, \lambda_{k}$ is the decay constant for group $k$, and $Q_{t o t}$ is the total volumetric heat source produced in the core as defined in Eq. (C.127). Considering axial velocity field, then Eq. (C.122) reduces to 


$$
\frac{\partial}{\partial t} h_{k}(r, z, t)+\frac{\partial}{\partial z}\left[u(z) h_{k}(r, z, t)\right]+\lambda_{k} h_{k}(r, z, t)=f_{k} Q_{t o t}(r, z, t)
$$

This equation can be solved in a similar way as the delayed neutron precursor equation analytical, semi-analytical, or numerical schemes. For the decay heat in the outer loop, there is no heat generated from fission, so the following equation is considered

$$
\frac{\partial}{\partial t} h_{k}(r, z, t)+\frac{\partial}{\partial z}\left[u(z) h_{k}(r, z, t)\right]+\lambda_{k} h_{k}(r, z, t)=0 .
$$

This equation should be solved to provide a heat source for the heat exchanger. The fission, decay, and total volumetric heat sources in the core can be expressed as

$$
\begin{gathered}
Q_{\text {decay }}(r, z, t)=\sum_{k=1}^{K_{D}} \lambda_{k} h_{k}(r, z, t), \\
Q_{\text {fiss }}(r, z, t)=\left[1-\sum_{k=1}^{K_{D}} f_{k}\right] \sum_{g=1}^{G} \varepsilon \sum_{f g}(r, z, t) \phi_{g}(r, z, t), \\
Q_{\text {tot }}(r, z, t)=\sum_{g=1}^{G} \varepsilon \sum_{f g}(r, z, t) \phi_{g}(r, z, t) \\
=Q_{\text {fiss }}(r, z, t)+Q_{\text {decay }}(r, z, t)
\end{gathered}
$$

where $\varepsilon$ is the recoverable energy release per fission. 


\section{Appendix D. PROTEUS-NODAL Input Data for MSR Analysis}

Various input cards have been introduced to PROTEUS-NODAL for MSR analyses. Table D.1 describes the added input cards to the input driver file. An input card for flowing fuel options and a card for the standalone thermal-hydraulics calculation options are provided.

Table D.1. Input Cards in Driver Input File

\begin{tabular}{|l|l|c|l|}
\hline \multicolumn{1}{|c|}{ Keyword } & \multicolumn{1}{|c|}{ Input Data } & $\begin{array}{c}\text { Default } \\
\text { value }\end{array}$ & Description \\
\hline Flowing Fuel Options & $\begin{array}{l}\text { YES } \\
\text { NO }\end{array}$ & NO & Activate the flowing fuel option \\
\hline USE_FLOWINGFUEL & $<$ Real Value $>$ & - & Transit time outside the core (s) \\
\hline TIME_OUT_CORE & $\begin{array}{l}\text { YES } \\
\text { NO }\end{array}$ & NO & $\begin{array}{l}\text { Activate MSR TH calculation. } \\
\text { Two XS files must be provided for } \\
\text { XS interpolation }\end{array}$ \\
\hline MSR Thermal-Hydraulics Options & $\begin{array}{l}\text { Unix file path to a MSR TH input } \\
\text { file }\end{array}$ \\
\hline USE_MSR_TH & Characters $>$ & $\begin{array}{l}\text { Unix file path to a cross section } \\
\text { data file at low temperature for XS } \\
\text { interpolation }\end{array}$ \\
\hline SourceFile_MSRTH & $\begin{array}{l}<\text { Max 128 } \\
\text { Characters }>\end{array}$ & - & $\begin{array}{l}\text { Unix file path to a cross section } \\
\text { data file at high temperature for XS } \\
\text { interpolation }\end{array}$ \\
\hline SOURCEFILE_XS_TEMP1
\end{tabular}

The key words added to the geometry input for the variational nodal $\mathrm{P}_{1}$ solver in $\mathrm{R}-\mathrm{\theta}-\mathrm{Z}$ geometry are described in Table D.2. The boundary condition for the radial boundary surface is specified with the keyword 'BOUNDARY_CONDITION' with input ' $\mathrm{R}$ '. The boundary conditions for the azimuthal boundary surfaces are specified with the keyword 'BOUNDARY_CONDITION' with input '- $T$ ' or ' $+\mathrm{T}$ ', where ' + ' is for the boundary of larger azimuthal angle and '-' is for the boundary of smaller azimuthal angle. 'EXTRAPOLATED' and 'VACUUM' can also be given in the $\theta$ direction. If the 'BOUNDARY_CONDITION' keyword is not specified, the periodic boundary condition is automatically applied in the $\theta$ direction. Note that for the periodic boundary condition, the total span of the $\theta$ grid specified in the 'TGRID' keyword should be one of the factors of 360. 
Table D.2. Keywords Added to Geometry Input for R- $\theta-Z$ Geometry

\begin{tabular}{|c|c|c|}
\hline Keyword & Input Data & Description \\
\hline BOUNDARY_CONDITION & R B.C. & \multirow{3}{*}{$\begin{array}{l}\text { B.C. = EXTRAPOLATED or } \\
\text { VACUUM }\end{array}$} \\
\hline BOUNDARY_CONDITION & -T B.C. & \\
\hline BOUNDARY_CONDITION & $+\mathrm{T}$ B.C. & \\
\hline RGRID & $r 1 r 2 \mathrm{n} r$ & $\begin{array}{l}r 1=\text { inner radius in } \mathrm{cm} \\
r 2=\text { outer radius in } \mathrm{cm} \\
\mathrm{n} r=\# \text { of nodes between } r 1 \text { and } r 2\end{array}$ \\
\hline TGRID & $\theta 1 \theta 2 \mathrm{n} \theta$ & $\begin{array}{l}\theta 1=\text { azimuthal angle at ' }-\mathrm{T} \text { ' boundary } \\
\theta 2=\text { azimuthal angle at ' }+\mathrm{T} \text { ' boundary } \\
\mathrm{n} \theta=\# \text { of nodes between } \theta 1 \text { and } \theta 2\end{array}$ \\
\hline
\end{tabular}

Table D.3 describes the added input cards to the material assignment file. This card specifies the flowing fuel velocity or the region to thermal-hydraulics channel. Table D.4 describes the input cards for the standalone thermal-hydraulics calculation. This includes the cards for general thermal-hydraulics properties of the core, the cards for cross section functionalization, the cards for heat exchanger parameters, the card for decay heat calculation model, the cards for heat conduction model in solid moderator, and the cards to activate transient scenarios.

Table D.3. Input Cards in Material Assignment File

\begin{tabular}{|l|l|l|l|}
\hline \multicolumn{2}{|c|}{ REGION_PROPERTY $1^{\text {st }} 2^{\text {nd }} \quad 3^{\text {rd }}$} & \multicolumn{1}{c|}{ Input Data } & \multicolumn{1}{c|}{ Description } \\
\hline REGION_PROPERTY & $1^{\text {st }}$ Value & $<$ Character Value $>$ & $\begin{array}{l}\text { Define a region to assign } \\
\text { properties }\end{array}$ \\
\cline { 2 - 4 } & $2^{\text {nd }}$ Value & $<$ Character Value $>$ & $\begin{array}{l}\text { Define the MSR property to } \\
\text { be assigned (VELOCITY, } \\
\text { FUELFLOW) }\end{array}$ \\
\cline { 2 - 5 } & $3^{\text {rd }}$ Value & $<$ Real Value $>$ & $\begin{array}{l}\text { Assign a value to selected } \\
\text { property (real value for } \\
\text { VELOCITY and 0.0 or } 1.0 \\
\text { for FUELFLOW) }\end{array}$ \\
\hline \multirow{2}{*}{ Note } & VELOCITY & $\begin{array}{l}\text { Required for regular MSR calculations to assign a } \\
\text { velocity value to each region (any real value can } \\
\text { be specified) (m/s) }\end{array}$ \\
\cline { 2 - 4 } & FUELFLOW & $\begin{array}{l}\text { Required for MSR thermal-hydraulics } \\
\text { calculations for region to flow channel mapping } \\
(1 \text { to be included or 0 to be excluded in the core } \\
\text { thermal-hydraulics calculations) }\end{array}$ \\
\hline
\end{tabular}


Table D.4. Input Cards in Standalone Thermal-Hydraulics Input File

\begin{tabular}{|c|c|c|c|}
\hline Keyword & Input Data & \begin{tabular}{|c|}
$\begin{array}{c}\text { Default } \\
\text { value }\end{array}$ \\
\end{tabular} & Description \\
\hline \multicolumn{4}{|l|}{ Core Parameters } \\
\hline TOTAL_POWER & $<$ Real Value $>$ & - & $\begin{array}{l}\text { Thermal power (W) for flux } \\
\text { and power normalization. It } \\
\text { should be equal to the value } \\
\text { of THERMAL_POWER in } \\
\text { the driver input file. }\end{array}$ \\
\hline INLET_TEMPERATURE & $<$ Real Value $>$ & - & Core inlet temperature $\left({ }^{\circ} \mathrm{C}\right)$ \\
\hline OUTLET_PRESSURE & $<$ Real Value $>$ & - & Core outlet pressure (MPa) \\
\hline MASS_FLOWRATE & $<$ Real Value $>$ & - & Inlet mass flow rate $(\mathrm{kg} / \mathrm{s})$ \\
\hline INLET_VELOCITY & $<$ Real Value $>$ & - & Inlet salt velocity (m/s) \\
\hline INITIAL_SALT_DENSITY & $<$ Real Value $>$ & - & Inlet fuel density $\left(\mathrm{kg} / \mathrm{m}^{3}\right)$ \\
\hline INLET_FLOWAREA & $<$ Real Value $>$ & - & Inlet flow area $\left(\mathrm{m}^{2}\right)$ \\
\hline ASSEMBLY_PITCH & $<$ Real Value $>$ & - & Assembly pitch (cm) \\
\hline ACTIVE_FUEL_HEIGHT & $<$ Real Value $>$ & - & Active fuel height $(\mathrm{cm})$ \\
\hline \multicolumn{4}{|c|}{ Temperature of Cross Sections Files } \\
\hline NUMBER_OF_XS_TEMPS & $<$ Integer Value $>$ & 2 & $\begin{array}{l}\text { Number of temperatures for } \\
\text { XS interpolation }\end{array}$ \\
\hline TEMPERTURE_1 & $<$ Real Value $>$ & - & $\begin{array}{l}\text { Temperature for the first XS } \\
\text { data file }\left({ }^{\circ} \mathrm{C}\right)\end{array}$ \\
\hline TEMPERTURE_2 & $<$ Real Value $>$ & - & $\begin{array}{l}\text { Temperature for the second } \\
\text { XS data file }\left({ }^{\circ} \mathrm{C}\right)\end{array}$ \\
\hline \multicolumn{4}{|l|}{ Heat Exchanger Parameters } \\
\hline OUTERLOOP_HX & $\begin{array}{l}\text { YES } \\
\text { NO }\end{array}$ & NO & $\begin{array}{l}\text { Activate the outer loop } \\
\text { model for heat exchanger } \\
\text { calculations }\end{array}$ \\
\hline HX_SALT_VELOCITY & $<$ Real Value $>$ & - & $\begin{array}{l}\text { Fuel salt velocity in heat } \\
\text { exchanger }(\mathrm{m} / \mathrm{s})\end{array}$ \\
\hline HX_TUBE_DIAMETER & $<$ Real Value $>$ & - & $\begin{array}{l}\text { Tube diameter of heat } \\
\text { exchanger }(\mathrm{m})\end{array}$ \\
\hline HX_TUBE_THICKNESS & $<$ Real Value $>$ & - & $\begin{array}{l}\text { Tube thickness of heat } \\
\text { exchanger }(\mathrm{m})\end{array}$ \\
\hline HX_LENGTH & $<$ Real Value $>$ & - & $\begin{array}{l}\text { Tube length of heat } \\
\text { exchanger (m) }\end{array}$ \\
\hline HX_NUMBER_OF_TUBES & $<$ Integer Value $>$ & - & $\begin{array}{l}\text { Number of tubes in heat } \\
\text { exchanger }\end{array}$ \\
\hline HX_INTERMEDIATE_LOOP & $\begin{array}{l}\text { YES } \\
\text { NO }\end{array}$ & NO & $\begin{array}{l}\text { Activate the intermediate } \\
\text { loop model for heat } \\
\text { exchanger calculations }\end{array}$ \\
\hline IT_INLET_TEMP & $<$ Real Value $>$ & - & $\begin{array}{l}\text { Inlet coolant temperature in } \\
\text { intermediate loop }\left({ }^{\circ} \mathrm{C}\right)\end{array}$ \\
\hline \multicolumn{4}{|l|}{ Decay Heat Calculation } \\
\hline DECAY_HEAT & YES & $\mathrm{NO}$ & Activate decay heat \\
\hline
\end{tabular}




\begin{tabular}{|c|c|c|c|}
\hline & NO & & calculations model \\
\hline \multicolumn{4}{|l|}{ Choose Transient Scenarios } \\
\hline UPOS & $\begin{array}{l}\text { YES } \\
\text { NO }\end{array}$ & NO & $\begin{array}{l}\text { Activate unprotected pump } \\
\text { over-speed transient }\end{array}$ \\
\hline ULOF & $\begin{array}{l}\text { YES } \\
\text { NO }\end{array}$ & NO & $\begin{array}{l}\text { Activate unprotected loss of } \\
\text { flow transient }\end{array}$ \\
\hline ULOHS & $\begin{array}{l}\text { YES } \\
\text { NO }\end{array}$ & NO & $\begin{array}{l}\text { Activate unprotected loss of } \\
\text { heat sink transient }\end{array}$ \\
\hline UFSOC & $\begin{array}{l}\text { YES } \\
\text { NO }\end{array}$ & NO & $\begin{array}{l}\text { Activate unprotected chilled } \\
\text { inlet transient }\end{array}$ \\
\hline Time Constant & $<$ Real Value $>$ & - & Transient time constant (s) \\
\hline \multicolumn{4}{|l|}{ Heat Conduction in Solid Moderator } \\
\hline SOLID_MODERATOR & $\begin{array}{l}\text { YES } \\
\text { NO }\end{array}$ & NO & $\begin{array}{l}\text { Activate the heat conduction } \\
\text { in solid moderator and the } \\
\text { flow splitting among parallel } \\
\text { fuel channels }\end{array}$ \\
\hline NATURAL_CIRCULATION & $\begin{array}{l}\text { YES } \\
\text { NO }\end{array}$ & NO & $\begin{array}{l}\text { Use the Nusselt number } \\
\text { correlation for natural } \\
\text { circulation }\end{array}$ \\
\hline MODERATOR_ISOTOPE & $\begin{array}{l}<\text { Character } \\
\text { Value }>\end{array}$ & - & $\begin{array}{l}\text { Solid moderator isotope for } \\
\text { thermal feedback calculation }\end{array}$ \\
\hline FUEL_FRACTION_CH & $<$ Real Value $>$ & 1.0 & $\begin{array}{l}\text { Fuel salt volume fraction in } \\
\text { a unit cell }\end{array}$ \\
\hline MODERATOR_FRACTION_CH & <Real Value $>$ & 0.0 & $\begin{array}{l}\text { Moderator volume fraction } \\
\text { in a unit cell }\end{array}$ \\
\hline NUMBER_OF_RADIAL_MESHES & <Integer Value> & - & $\begin{array}{l}\text { Number of radial meshes for } \\
\text { heat conduction calculation }\end{array}$ \\
\hline MOD_GAMMA_HEAT_FACT & $<$ Real Value $>$ & 0.0 & $\begin{array}{l}\text { Fraction of heat deposit in } \\
\text { the solid moderator due to } \\
\text { gamma heating }\end{array}$ \\
\hline
\end{tabular}


Figures D.1, D.2, and D.3 show sample input files of the driver input, assignment input, and standalone thermal-hydraulics calculation input, respectively.

\begin{tabular}{|c|c|}
\hline THERMAL_POWER & $3.0000 \mathrm{E}+09$ \\
\hline MAX_FISSION_ITER & 500 \\
\hline EIGENVALUE_GUESS & 1.0 \\
\hline TOLERANCE_EIGENVALUE & $1.0 \mathrm{e}-5$ \\
\hline TOLERANCE_FISSION & $1.0 \mathrm{e}-5$ \\
\hline TOLERANCE_FLUX & $1.0 \mathrm{e}-5$ \\
\hline SOURCEFILE_XS & MSFR-U233 . ISOTXS \\
\hline SOURCEFILE_DELAY & MSFR - U233. DLAYXS \\
\hline SOURCEFILE_MATERIAL & MSFR_U233.assignment \\
\hline SOURCEFILE_GRID & MSFR_U233.grid \\
\hline USE_TRANSPORT & YES \\
\hline USE_CMFD_ACCEL & YES \\
\hline CMFD_SOLVER_BACKVECTORS & 20 \\
\hline CMFD_UNDERRELAXATION & 0.5 \\
\hline EXPORT_SOLUTION_VTK & YES \\
\hline USE_FLOWINGFUEL & YES \\
\hline TIME_OUT_CORE & 2.0 \\
\hline USE_MSR_TH & YES \\
\hline SourceFile_MSRTH & MSFR_TH_Input.TH \\
\hline SOURCEFILE_XS_TEMP1 & MSFR-U233-900K. ISOTXS \\
\hline SOURCEFILE_XS_TEMP2 & MSFR-U233-1200K. ISOTXS \\
\hline
\end{tabular}

Fig. D.1. Example of a driver input file. 


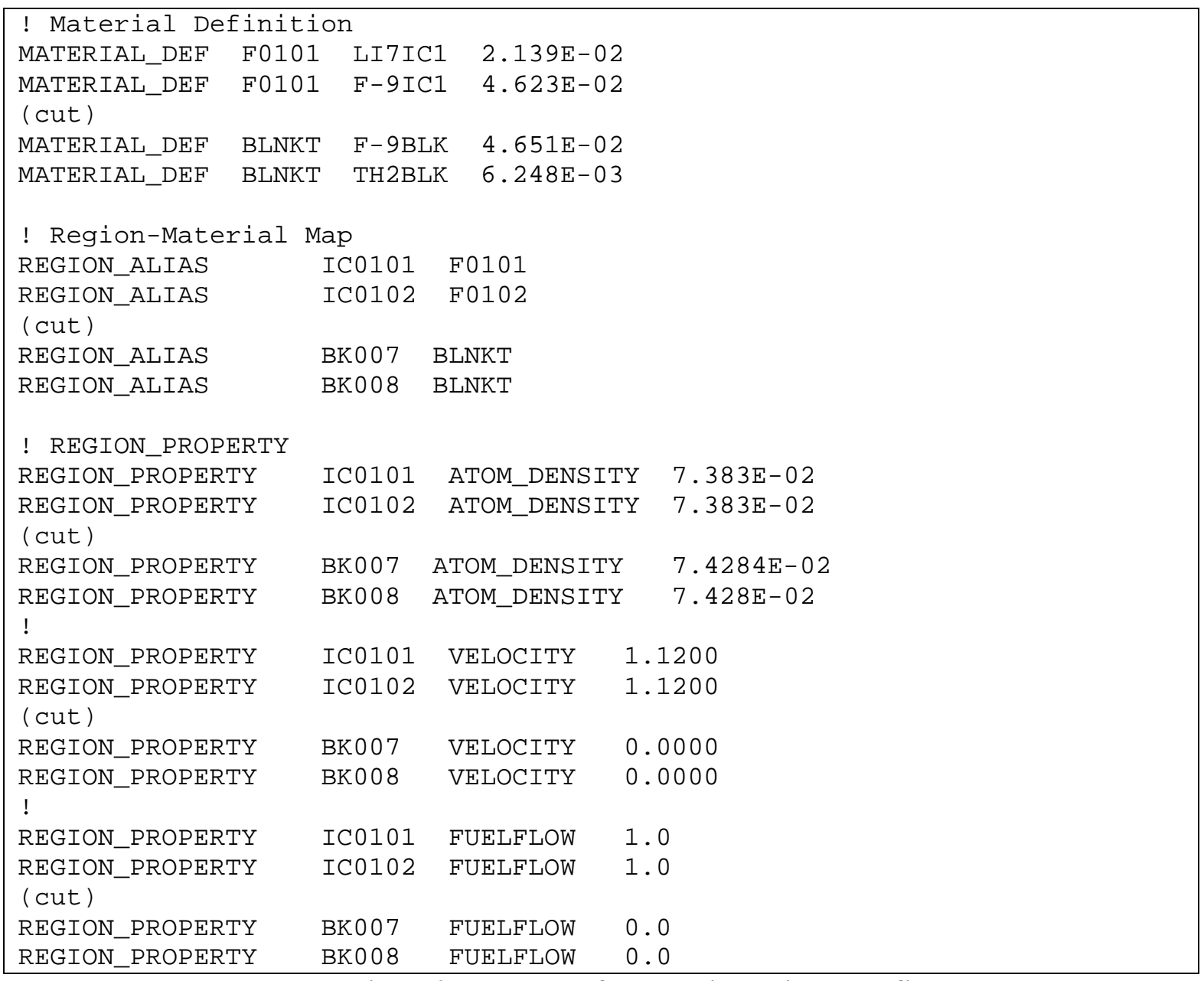

Fig. D.2. Example of a material assignment file. 


\begin{tabular}{|c|c|}
\hline TOTAL_POWER & $3.00 E+09$ \\
\hline INLET_TEMPERATURE & 650.0 \\
\hline OUTLET_PRESSURE & $\odot .101325$ \\
\hline MASS_FLOWRATE & $1.8772 \mathrm{E}+\odot 4$ \\
\hline INLET_VELOCITY & 1.1275 \\
\hline INITIĀL_SALT_DENSITY & 4168.84 \\
\hline INLET_FLOWAREA & 3.9938 \\
\hline ASSEMBLY_PITCH & 22.5115 \\
\hline ACTIVE_FUEL_HEIGHT & 225.500 \\
\hline NUMBER_OF_XS_TEMPS & 2 \\
\hline TEMPERTURE_1 & 626.85 \\
\hline TEMPERTURE_2 & 926.85 \\
\hline SOLID_MODERATOR & YES \\
\hline MODERATOR_ISOTOPE & C \\
\hline NATURAL_CIRCULATION & NO \\
\hline FUEL_FRACTION_CH & $\odot .2229$ \\
\hline MODERATOR_FRACTION_CH & 0.7771 \\
\hline NUMBER_OF_RADIAL_MESHES & 5 \\
\hline MOD_GAMMA_HEAT_FACT & $\odot .05$ \\
\hline OUTERLOOP_HX & YES \\
\hline HX_NUMBER_OF_TUBES & 8061 \\
\hline HX_SALT_VELOCITY & 2.780 \\
\hline HX_TUBE_DIAMETER & 0.004 \\
\hline HX_TUBE_THICKNESS & $\odot .000$ \\
\hline HX_LENGTH & 2.000 \\
\hline HX_INTERMEDIATE_LOOP & YES \\
\hline IT_INLET_TEMP & $550.0 \odot$ \\
\hline DECAY_HEAT & NO \\
\hline ULOF & NO \\
\hline UPOS & YES \\
\hline ULOHS & NO \\
\hline UFSOC & NO \\
\hline TRANSIENT_TIME_CONSTANT & 5.0 \\
\hline
\end{tabular}

Fig. D.3. Example of an assignment file for standalone thermal-hydraulics calculation. 


\section{Appendix E. Input Data for TreeFrog and TreeKangaroo Applications}

The applications "TreeFrog” and "TreeKangaroo" use the MOOSE style block-structured input file format. Each block is identified with square brackets. A block can contain multiple sub-blocks. Each sub-block starts with "[./sub-block name]" and ends with "[../]". Within each block or sub-block, the line inputs are given as pairs of parameter and value with an equal sign between them. The input data blocks are described in the following subsections, and then sample input files are presented.

\section{E.1. Description of Input Data Blocks}

\section{E.1.1. Mesh}

The Mesh block is used to generate the mesh to be consistent with the SAM mesh. Table E.1 describes the input parameters of the mesh block. The TreeKangaroo and TreeFrog meshes are generated through the MOOSE function "GeneratedMesh". The mesh dimension is 1 if there is only 1 channel in the SAM input. If the number of channels is greater than 1 , the mesh dimension is 2 . The parameter " $\mathrm{nx}$ " is the number of axial elements in the core channels in the SAM input. The parameter "ny" is the number of core channels in the SAM input minus one.

Table E.1. Input Description of Mesh Block

\begin{tabular}{|l|l|c|l|}
\hline \multicolumn{1}{|c|}{ Keyword } & \multicolumn{1}{|c|}{ Input Data } & $\begin{array}{c}\text { Default } \\
\text { value }\end{array}$ & \multicolumn{1}{c|}{ Description } \\
\hline [Mesh] & GeneratedMesh & - & MOOSE function for mesh generation \\
\hline type & 1 & - & $\begin{array}{l}\text { 1 if the SAM input has only one channel. } \\
\text { Otherwise, 2. }\end{array}$ \\
\hline dim & 2 & - & $\begin{array}{l}\text { Number of axial elements in core channels in the } \\
\text { SAM input }\end{array}$ \\
\hline nx & unsigned int & - & $\begin{array}{l}\text { Only used when dim = 2. Number of core } \\
\text { channels in the SAM input minus one }\end{array}$ \\
\hline ny & unsigned int & & \\
\hline[]
\end{tabular}

\section{E.1.2. AuxVariables}

The AuxVariables (auxiliary variable) block is used to store the power density, temperature, velocity, and density in the TreeKangaroo and TreeFrog meshes. Table E.2 describes the input parameters of the auxiliary variable block. In the MOOSE framework, auxiliary variables can be defined in Element or Nodal. However, in the TreeKangaroo and TreeFrog meshes, only the first order nodal auxiliary variables are utilized. Each auxiliary variable is defined in a sub-block and the name of the sub-block is the name of this auxiliary variable. In TreeKangaroo and TreeFrog, at least four auxiliary variables need to be defined to store the power density, temperature, velocity, and density data in each application. To transfer the data between two applications, the auxiliary variable defined in other application need to be listed as a sub-block if data is transferred from this auxiliary variable. In the sub- 
block for this kind of auxiliary variable, only [./sub-block name] and [../] are specified and no detailed parameter is needed.

Table E.2. Input Description of AuxVariables Block

\begin{tabular}{|l|l|l|l|}
\hline \multicolumn{1}{|c|}{ Keyword } & \multicolumn{1}{|c|}{ Input Data } & $\begin{array}{c}\text { Default } \\
\text { value }\end{array}$ & \multicolumn{1}{|c|}{ Description } \\
\hline $\begin{array}{l}\text { [AuxVariables] } \\
\text { [./AuxVariables1] }\end{array}$ & FIRST & - & The first order auxiliary variable is used \\
\hline order & LAGRANGE & - & The nodal auxiliary variable is used \\
\hline family & - & $\begin{array}{l}\text { Initial value for this variable, only used in } \\
\text { steady state simulations }\end{array}$ \\
\hline initial_condition & double & \multicolumn{2}{|l}{} \\
\hline $\begin{array}{l}{[. . /]} \\
{[]}\end{array}$
\end{tabular}

\section{E.1.3. Function}

The Function block is used to define functions depending on spatial position and time only. It is widely used in defining initial conditions, boundary conditions, and other quantities in MOOSE applications. Table E.3 shows two example functions: a function created by a mathematical expression and a piecewise constant function. Other available function forms can be found in the MOOSE website.

Table E.3. Input Description of Function Block

\begin{tabular}{|c|c|c|c|}
\hline Keyword & Input Data & $\begin{array}{l}\text { Default } \\
\text { value }\end{array}$ & Description \\
\hline \multicolumn{4}{|c|}{$\begin{array}{l}\text { [Function] } \\
\text { [./ParsedFunction] }\end{array}$} \\
\hline type & ParsedFunction & - & Function created by a mathematical expression \\
\hline value & LAGRANGE & - & User defined function depending on $\mathrm{t}, \mathrm{x}, \mathrm{y}, \mathrm{z}$ \\
\hline \multicolumn{4}{|r|}{ 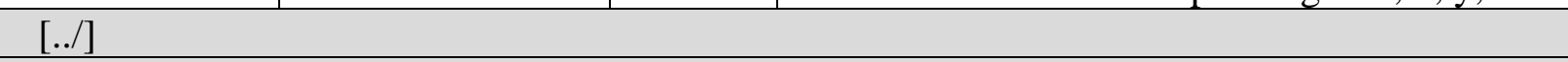 } \\
\hline \multicolumn{4}{|c|}{ [./PiecewiseConstant] } \\
\hline type & PiecewiseConstant & - & Defines data using a set of $x-y$ data pairs \\
\hline axis & $\mathrm{x} \mathrm{y} \mathrm{z}$ & - & $\begin{array}{l}\text { The axis used ( } \mathrm{x}, \mathrm{y}, \text { or } \mathrm{z}) \text { if this is to be a } \\
\text { function of position }\end{array}$ \\
\hline data_file & FileName & - & $\begin{array}{l}\text { File holding comma-separated value (csv) data } \\
\text { for use with Piecewise }\end{array}$ \\
\hline format & $\begin{array}{l}\text { columns } \\
\text { rows }\end{array}$ & - & $\begin{array}{l}\text { Format of csv data file that is in either in } \\
\text { columns or rows }\end{array}$ \\
\hline scale_factor & double & - & Scale factor to be applied to the ordinate values \\
\hline $\mathrm{X}$ & vector & - & The abscissa values \\
\hline x_index_in_file & unsigned int & 0 & The abscissa index in the data file \\
\hline xy_data & vector & - & $\begin{array}{l}\text { All function data, supplied in abscissa and } \\
\text { ordinate pairs }\end{array}$ \\
\hline xy_in_file_only & bool & True & $\begin{array}{l}\text { If the data file contains only abscissa and } \\
\text { ordinate data }\end{array}$ \\
\hline
\end{tabular}




\begin{tabular}{|l|l|c|l|}
\hline y & vector & - & The ordinate values \\
\hline y_index_in_file & unsigned int & 1 & The ordinate index in the data file \\
\hline direction & $\begin{array}{l}\text { left } \\
\text { right }\end{array}$ & left & Direction to look to find value: left, right \\
\hline$[. . /]$ & \multicolumn{3}{|l|}{} \\
\hline$\left[\begin{array}{l}\mid l \\
\hline\end{array}\right.$
\end{tabular}

\section{E.1.4. Problem}

The Problem block is designed to hold the numerical systems that are ultimately solved. Tables E.4 and E.5 show the problem blocks of TreeKangaroo and TreeFrog, respectively. Since there is no equation system solved in TreeKangaroo, the "solve" option is set to false. In TreeFrog, there are two types of problems supported: "ProteusSP3Problem" and "ProteusP1Problem”. They are all derived from ExternalProblem.

Table E.4. Input Description for Problem Block in TreeKangaroo

\begin{tabular}{|l|l|c|l|}
\hline \multicolumn{1}{|c|}{ Keyword } & \multicolumn{1}{|c|}{ Input Data } & $\begin{array}{c}\text { Default } \\
\text { value }\end{array}$ & \multicolumn{1}{c|}{ Description } \\
\hline [Problem] & FEProblem & - & MOOSE Problem type in TreeKangaroo \\
\hline type & false & - & $\begin{array}{l}\text { Whether or not actually solve the } \\
\text { nonlinear system. In TreeKangaroo, it is } \\
\text { always set to false. }\end{array}$ \\
\hline $\begin{array}{l}\text { restart_file } \\
\text { base }\end{array}$ & FileNameNoExtension & - & $\begin{array}{l}\text { File base name used for restart } \\
\text { (e.g. / or /LATEST to grab the latest file } \\
\text { available). Used when restart transient } \\
\text { calculation from steady state solution }\end{array}$ \\
\hline $\begin{array}{l}\text { skip_additional } \\
\text { restart_data }\end{array}$ & bool & False & $\begin{array}{l}\text { True to skip additional data in equation } \\
\text { system for restart. It needs to be true for } \\
\text { starting a transient calculation with a } \\
\text { steady state solution. }\end{array}$ \\
\hline [] & &
\end{tabular}


Table E.5. Input Description for Problem Block in TreeFrog

\begin{tabular}{|c|c|c|c|}
\hline Keyword & Input Data & $\begin{array}{c}\text { Default } \\
\text { value }\end{array}$ & Description \\
\hline \multicolumn{4}{|l|}{ [Problem] } \\
\hline type & \begin{tabular}{|l|} 
ProteusSP3Problem \\
ProteusP1Problem
\end{tabular} & - & $\begin{array}{l}\text { Use PROTEUS-NODAL SP3 solver or } \\
\text { P1 solver }\end{array}$ \\
\hline problem_type & $\begin{array}{l}\text { Steady } \\
\text { Transient }\end{array}$ & Steady & $\begin{array}{l}\text { Specify a steady state or transient } \\
\text { problem }\end{array}$ \\
\hline $\begin{array}{l}\text { sync_to_mesh } \\
\text { _power }\end{array}$ & string & - & $\begin{array}{l}\text { The auxiliary variable name to hold the } \\
\text { power density data in TreeFrog }\end{array}$ \\
\hline $\begin{array}{l}\text { sync_from_mesh } \\
\text { temperature }\end{array}$ & string & - & $\begin{array}{l}\text { The auxiliary variable name to hold the } \\
\text { temperature data from SAM in TreeFrog }\end{array}$ \\
\hline $\begin{array}{l}\text { sync_from_mesh } \\
\text { _velocity }\end{array}$ & string & - & $\begin{array}{l}\text { The auxiliary variable name to hold the } \\
\text { velocity data from SAM in TreeFrog }\end{array}$ \\
\hline $\begin{array}{l}\text { sync_from_mesh } \\
\text { _density }\end{array}$ & string & - & $\begin{array}{l}\text { The auxiliary variable name to hold the } \\
\text { density data from SAM in TreeFrog }\end{array}$ \\
\hline $\begin{array}{l}\text { total_node } \\
\text { number }\end{array}$ & unsigned int & - & $\begin{array}{l}\text { The number of assemblies in PROTEUS- } \\
\text { NODAL }\end{array}$ \\
\hline $\begin{array}{l}\text { total_plane } \\
\text { number }\end{array}$ & unsigned int & - & $\begin{array}{l}\text { The number of axial planes in } \\
\text { PROTEUS-NODAL }\end{array}$ \\
\hline $\begin{array}{l}\text { flow_plane } \\
\text { down }\end{array}$ & unsigned int & - & $\begin{array}{l}\text { The first flow plane number in } \\
\text { PROTEUS-NODAL }\end{array}$ \\
\hline flow_plane_up & unsigned int & - & $\begin{array}{l}\text { The last flow plane number in } \\
\text { PROTEUS-NODAL }\end{array}$ \\
\hline $\begin{array}{l}\text { channel_total } \\
\text { number }\end{array}$ & unsigned int & - & $\begin{array}{l}\text { Total number of core channels in the } \\
\text { SAM input }\end{array}$ \\
\hline channel_number & vector & - & $\begin{array}{l}\text { The corresponding channel number in } \\
\text { SAM of each PROTEUS-NODAL } \\
\text { assembly. If } 0 \text { is set for an assembly, this } \\
\text { assembly is not included in the SAM } \\
\text { model (not flowing assembly) }\end{array}$ \\
\hline geometry_type & $\begin{array}{l}\text { RZ } \\
\text { Hex }\end{array}$ & - & $\begin{array}{l}\text { Only use when problem type is } \\
\text { ProteusP1Problem. It provides whether } \\
\text { the geometry in PROTEUS-NODAL } \\
\text { input is RZ or Hexagonal }\end{array}$ \\
\hline r_grid & vector & - & $\begin{array}{l}\text { Only use when geometry_type is RZ. } \\
\text { The first value is the inner radius of first } \\
\text { radial node. Other value is the outer } \\
\text { radius of each radial node }\end{array}$ \\
\hline Г] & & & \\
\hline
\end{tabular}




\section{E.1.5. Executioner}

The Executioner block specifies how the simulation will be executed. It includes the commands to control the solver behavior and time stepping. In TreeKangaroo and TreeFrog, three kinds of executioner are utilized: "Steady", "SteadyWithPicardCheck", and "Transient". Tables E.6, E.7, and E.8 show the input descriptions for these three executioner blocks. There are many options available in these executioners, but only the options used in TreeKangaroo and TreeFrog are listed. The complete list of available options of these executioners can be found in the MOOSE website. A steady state calculation is usually performed with the Steady Executioner in TreeFrog and with the SteadyWithPicardCheck Executioner in TreeKangaroo. In the Transient executioner, the TimeSteppers system is usually used as a sub-block in Executioner block to control how to move through time (i.e., to select the time step size). FuctionDT is usually used in TreeKangaroo and TreeFrog.

Table E.6. Input Description for Steady Executioner Block

\begin{tabular}{|c|c|c|c|}
\hline Keyword & Input Data & Default value & Description \\
\hline \multicolumn{4}{|l|}{ [Executioner] } \\
\hline type & Steady & - & Steady Executioner used in TreeFrog \\
\hline
\end{tabular}

Table E.7. Input Description for SteadyWithPicardCheck Executioner Block

\begin{tabular}{|c|c|c|c|}
\hline Keyword & Input Data & $\begin{array}{l}\text { Default } \\
\text { value }\end{array}$ & Description \\
\hline \multicolumn{4}{|l|}{ [Executioner] } \\
\hline type & SteadyWithPicardCheck & - & $\begin{array}{l}\text { Use Picard iteration in Steady } \\
\text { calculation }\end{array}$ \\
\hline $\begin{array}{l}\text { disable_picard } \\
\text { _residual_norm } \\
\text { _check }\end{array}$ & True & - & $\begin{array}{l}\text { Disable the default Picard } \\
\text { residual norm evaluation of } \\
\text { MOOSE }\end{array}$ \\
\hline picard_max_its & unsigned int & 1 & $\begin{array}{l}\text { Specifies the maximum number } \\
\text { of Picard iterations }\end{array}$ \\
\hline pp_name & PostprocessorName & - & $\begin{array}{l}\text { Postprocessor for custom Picard } \\
\text { convergence check }\end{array}$ \\
\hline pp_step_tol & double & - & $\begin{array}{l}\text { The relative difference of the } \\
\text { postprocessor values between } \\
\text { two iterations to shoot for } \\
\text { during Picard iterations }\end{array}$ \\
\hline
\end{tabular}

Table E.8. Input Description for Transient Executioner Block

\begin{tabular}{|l|l|c|l|}
\hline \multicolumn{1}{|c|}{ Keyword } & \multicolumn{1}{|c|}{ Input Data } & Default value & \multicolumn{1}{c|}{ Description } \\
\hline [Executioner] & - & Executioner for time varying simulations \\
\hline type & Transient & - & The start time of the simulation \\
\hline start_time & double & 0 &
\end{tabular}




\begin{tabular}{|l|l|c|l|}
\hline end_time & double & $1 \mathrm{E}+30$ & The end time of the simulation \\
\hline $\mathrm{dt}$ & double & 1 & The time step size between solves \\
\hline \multicolumn{2}{|c|}{ [./TimeStepper] } & - & $\begin{array}{l}\text { Timestepper whose steps vary over time } \\
\text { according to a user-defined function }\end{array}$ \\
\hline type & FunctionDT & - & $\begin{array}{l}\text { The name of the time-dependent function } \\
\text { that prescribes the time step size }\end{array}$ \\
\hline function & FunctionName & -
\end{tabular}

\section{E.1.6. MultiApps}

The MultiApps (multi-application) block is used in a master application to specify the parameters for sub-applications. The parameters for each sub-application are included in a sub-block of MultiApps block. Table E.9 shows the input description for MultiApps block. Two types of MultiApps can be used in TreeKangaroo: "FullSolveMultiApp" for steady state calculations and "TransientMultiApp" for transient calculations. FullSolveMultiApp performs a complete simulation during each execution, so it is used in the steady state calculations. TransientMultiApp is designed to perform simulations with sub-applications that progress in time with the master application, which is used in the transient calculations.

Table E.9. Input Description for MultiApps Block

\begin{tabular}{|c|c|c|c|}
\hline Keyword & Input Data & $\begin{array}{l}\text { Default } \\
\text { value }\end{array}$ & Description \\
\hline \multicolumn{4}{|l|}{$\begin{array}{r}\text { [MultiApps] } \\
\text { [./sub-app] }\end{array}$} \\
\hline type & $\begin{array}{l}\text { FullSolveMultiApp } \\
\text { TransientMultiApp }\end{array}$ & - & $\begin{array}{l}\text { FullSolveMultiApp for steady state } \\
\text { calculations and TransientMultiApp } \\
\text { for transient calculations }\end{array}$ \\
\hline app_type & $\begin{array}{l}\text { treefrogApp } \\
\text { SamApp }\end{array}$ & - & $\begin{array}{l}\text { The type of application to build } \\
\text { (applications not registered can be } \\
\text { loaded with dynamic libraries). Master } \\
\text { application type will be used if not } \\
\text { provided. }\end{array}$ \\
\hline input_files & FileName & - & The input file for each application \\
\hline library_path & string & - & Path to search for dynamic libraries \\
\hline execute_on & $\begin{array}{l}\text { NONE, INITIAL, LINEAR, } \\
\text { NONLINEAR, } \\
\text { TIMESTEP_END, } \\
\text { TIMESTEP_BEGIN, FINAL, } \\
\text { CUSTOM }\end{array}$ & $\begin{array}{l}\text { TIMESTEP } \\
\text { _BEGIN }\end{array}$ & $\begin{array}{l}\text { Indicate when this subapp should be } \\
\text { executed }\end{array}$ \\
\hline sub_cycling & bool & False & $\begin{array}{l}\text { Set to true to allow this MultiApp to } \\
\text { take smaller time steps than the master }\end{array}$ \\
\hline \multicolumn{4}{|l|}{$[. . /]$} \\
\hline
\end{tabular}




\section{E.1.7. Transfers}

Transfer objects in MOOSE are designed to move data to and from the sub-applications. Table E.10 presents the input description for transfer block. The data transfer between TreeKangaroo and TreeFrog is performed through MultiAppCopyTransfer. The data transfer between TreeKangaroo and SAM is carried out by MultiAppVPPTransferFromSam and MultiAppPowerTransferToSam.

Table E.10. Input Description for Transfers Block

\begin{tabular}{|c|c|c|c|}
\hline Keyword & Input Data & $\begin{array}{l}\text { Default } \\
\text { value }\end{array}$ & Description \\
\hline \multicolumn{4}{|c|}{\begin{tabular}{|l} 
[Transfers] \\
[./MultiAppCopyTransfer]
\end{tabular}} \\
\hline type & MultiAppCopyTransfer & - & $\begin{array}{l}\text { The meshes in the master } \\
\text { and sub application must be } \\
\text { identical }\end{array}$ \\
\hline direction & $\begin{array}{l}\text { to_multiapp } \\
\text { from_multiapp }\end{array}$ & - & $\begin{array}{l}\text { Whether this transfer will } \\
\text { be 'to' or 'from' a MultiApp. } \\
\text { Bidirectional when both } \\
\text { from_multiapp and } \\
\text { to_multiapp are provided }\end{array}$ \\
\hline multi_app & MultiAppName & - & $\begin{array}{l}\text { The name of the MultiApp } \\
\text { to use }\end{array}$ \\
\hline $\begin{array}{l}\text { Source } \\
\text { variable }\end{array}$ & vector & - & $\begin{array}{l}\text { The variable to transfer } \\
\text { from }\end{array}$ \\
\hline variable & vector & - & $\begin{array}{l}\text { The auxiliary variable to } \\
\text { store the transferred values } \\
\text { in }\end{array}$ \\
\hline execute_on & $\begin{array}{l}\text { NONE, INITIAL, LINEAR, } \\
\text { NONLINEAR, TIMESTEP_END, } \\
\text { TIMESTEP_BEGIN, FINAL, } \\
\text { CUSTOM }\end{array}$ & $\begin{array}{l}\text { Same as } \\
\text { MultiApps }\end{array}$ & $\begin{array}{l}\text { Indicate when this transfer } \\
\text { function should be executed }\end{array}$ \\
\hline \multicolumn{4}{|l|}{$[. . /]$} \\
\hline \multicolumn{4}{|c|}{ [./MultiAppPowerTransferToSam] } \\
\hline type & $\begin{array}{l}\text { MultiAppPowerTransferToS } \\
\text { am }\end{array}$ & - & $\begin{array}{l}\text { Transfer power density } \\
\text { from TreeKangaroo to } \\
\text { SAM }\end{array}$ \\
\hline direction & $\begin{array}{l}\text { to_multiapp } \\
\text { from_multiapp }\end{array}$ & - & $\begin{array}{l}\text { Whether this Transfer will } \\
\text { be 'to' or 'from' a } \\
\text { MultiApp, or bidirectional, } \\
\text { by providing both } \\
\text { FROM_MULTIAPP and } \\
\text { TO_MULTIAPP }\end{array}$ \\
\hline multi_app & SAM MultiAppName & - & $\begin{array}{l}\text { The name of the SAM } \\
\text { MultiApp specified in } \\
\text { MultiApps block }\end{array}$ \\
\hline
\end{tabular}




\begin{tabular}{|c|c|c|c|}
\hline proteus_power & VariableName & - & $\begin{array}{l}\text { The name of the Aux } \\
\text { variable to transfer the } \\
\text { power density value from }\end{array}$ \\
\hline sam_power & VariableName & - & $\begin{array}{l}\text { The name of the Aux } \\
\text { variable to transfer the } \\
\text { power density value to }\end{array}$ \\
\hline channel_index & unsigned int & - & $\begin{array}{l}\text { The channel index in SAM. } \\
\text { The channels are ordered } \\
\text { from center to outer, i.e. } \\
\text { the index for center } \\
\text { channel is } 1\end{array}$ \\
\hline $\begin{array}{l}\text { channel_total_ } \\
\text { number }\end{array}$ & unsigned int & - & $\begin{array}{l}\text { Total core channel number } \\
\text { in SAM input }\end{array}$ \\
\hline elem_begin & unsigned int & - & $\begin{array}{l}\text { The id for first element in } \\
\text { SAM mesh of this channel }\end{array}$ \\
\hline elem_end & unsigned int & - & $\begin{array}{l}\text { The id for last element in } \\
\text { SAM mesh of this channel }\end{array}$ \\
\hline execute_on & $\begin{array}{l}\text { NONE, INITIAL, LINEAR, } \\
\text { NONLINEAR, TIMESTEP_END, } \\
\text { TIMESTEP_BEGIN, FINAL, } \\
\text { CUSTOM }\end{array}$ & $\begin{array}{l}\text { Same as } \\
\text { MultiApps }\end{array}$ & $\begin{array}{l}\text { Indicate when this transfer } \\
\text { function should be } \\
\text { executed }\end{array}$ \\
\hline \multicolumn{4}{|c|}{ 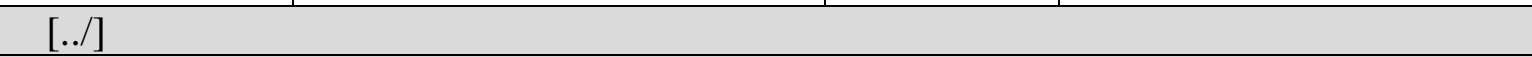 } \\
\hline \multicolumn{4}{|c|}{ [./MultiAppVPPTransferFromSam] } \\
\hline type & $\begin{array}{l}\text { MultiAppVPPTransferFrom } \\
\text { Sam }\end{array}$ & - & $\begin{array}{l}\text { Transfer temperature, } \\
\text { velocity, density from } \\
\text { SAM }\end{array}$ \\
\hline direction & $\begin{array}{l}\text { to_multiapp } \\
\text { from_multiapp }\end{array}$ & - & $\begin{array}{l}\text { Whether this Transfer will } \\
\text { be 'to' or 'from' a } \\
\text { MultiApp, or bidirectional, } \\
\text { by providing both } \\
\text { FROM_MULTIAPP and } \\
\text { TO_MULTIAPP }\end{array}$ \\
\hline multi_app & SAM MultiAppName & - & $\begin{array}{l}\text { The name of the SAM } \\
\text { MultiApp specified in } \\
\text { MultiApps block }\end{array}$ \\
\hline $\begin{array}{l}\text { vector_postpro } \\
\text { cessor }\end{array}$ & VectorPostprocessorName & - & $\begin{array}{l}\text { The name of the } \\
\text { VectorPostprocessor in } \\
\text { SAM input to transfer the } \\
\text { data from }\end{array}$ \\
\hline variable_vpp & vector & & $\begin{array}{l}\text { The name of variables in } \\
\text { SAM that the } \\
\text { VectorPostprocessor } \\
\text { operates on }\end{array}$ \\
\hline variable & vector & & $\begin{array}{l}\text { The name of aux variable } \\
\text { to store the transferred } \\
\text { data. The sequence of the }\end{array}$ \\
\hline
\end{tabular}




\begin{tabular}{|l|l|l|l|}
\hline & & & $\begin{array}{l}\text { aux variables in this input } \\
\text { is the same as the sequence } \\
\text { in variable_sam }\end{array}$ \\
\hline channel_index & unsigned int & - & $\begin{array}{l}\text { The channel index in SAM. } \\
\text { The channels are ordered } \\
\text { from center to outer, i.e. } \\
\text { the index for center } \\
\text { channel is 1 }\end{array}$ \\
\hline $\begin{array}{l}\text { channel_total_- } \\
\text { number }\end{array}$ & unsigned int & $\begin{array}{l}\text { Total core channel number } \\
\text { in SAM input }\end{array}$ \\
\hline $\begin{array}{l}\text { [../] } \\
{[]}\end{array}$ & - & \\
\hline
\end{tabular}

\section{E.1.8. Outputs}

The Output block is designed to set different output types. Table E.11 shows the input description of output block. The output types used in TreeKangaroo and TreeFrog are Console, Checkpoint, and CSV. The Console type is to output to screen. The Checkpoint is the MOOSE internal format used for restart and recovery. The CSV is to write the postprocessor and scalar variables to a CSV file. Additional output types supported in the MOOSE framework can be found in the MOOSE website. To activate a certain output type, MOOSE allows two ways. One is the "short-cut" syntax in the output block shown in Table E.11. In this case, the output type is activated by setting the output short-cut to True. The other is the "sub-block" syntax shown in Table E.12. Within each sub-block, there are options to control how to output data.

Table E.11. Input Description for Outputs Block with Short-cut Syntax

\begin{tabular}{|c|c|c|c|}
\hline Keyword & Input Data & Default value & Description \\
\hline \multicolumn{4}{|l|}{ [Outputs] } \\
\hline console & bool & - & Short-cut syntax for Console output \\
\hline checkpoint & bool & - & Short-cut syntax for Checkpoint output \\
\hline CSV & bool & - & Short-cut syntax for CSV output \\
\hline
\end{tabular}

Table E.12. Input Description for Outputs Block with Sub-block Syntax

\begin{tabular}{|l|l|c|l|}
\hline \multicolumn{1}{|c|}{ Keyword } & \multicolumn{1}{|c|}{ Input Data } & Default value & Description \\
\hline $\begin{array}{l}\text { [Outputs] } \\
\text { [./Console] }\end{array}$ & Console & - & $\begin{array}{c}\text { Write to the screen and } \\
\text { optionally a file }\end{array}$ \\
\hline type & string & - & $\begin{array}{l}\text { Desired solution output name without } \\
\text { extension. If not provided, MOOSE } \\
\text { sets the output file name of master app } \\
\text { to the input file name of master app } \\
\text { and sets the output file name of } \\
\text { subapp to the input file name of }\end{array}$ \\
\hline
\end{tabular}




\begin{tabular}{|c|c|c|c|}
\hline & & & master app plus the subapp name. \\
\hline output_file & bool & False & Output to the file \\
\hline output_screen & bool & True & Output to the screen \\
\hline \multicolumn{4}{|l|}{ [../] } \\
\hline \multicolumn{4}{|l|}{ [./CSV] } \\
\hline type & CSV & - & $\begin{array}{l}\text { Output for postprocessors, } \\
\text { vector postprocessors, and scalar } \\
\text { variables using CSV }\end{array}$ \\
\hline file_base & string & - & $\begin{array}{l}\text { Desired solution output name without } \\
\text { extension. If not provided, MOOSE } \\
\text { sets the output file name of master app } \\
\text { to the input file name of master app } \\
\text { and sets the output file name of } \\
\text { subapp to the input file name of } \\
\text { master app plus the subapp name. }\end{array}$ \\
\hline execute_on & $\begin{array}{l}\text { NONE, INITIAL, } \\
\text { LINEAR, } \\
\text { NONLINEAR, } \\
\text { TIMESTEP_END, } \\
\text { TIMESTEP_BEGIN, } \\
\text { FINAL, CUSTTM }\end{array}$ & $\begin{array}{c}\text { INITIAL, } \\
\text { TIMESTEP_EN } \\
\text { D }\end{array}$ & $\begin{array}{l}\text { Indicate when this output should be } \\
\text { executed }\end{array}$ \\
\hline start_step & int & - & $\begin{array}{l}\text { Time step at which this output object } \\
\text { begins to operate }\end{array}$ \\
\hline end_step & int & - & $\begin{array}{l}\text { Time step at which this output object } \\
\text { stop operating }\end{array}$ \\
\hline time_column & bool & True & $\begin{array}{l}\text { Whether or not the 'time' column } \\
\text { should be written for Postprocessor } \\
\text { CSV files }\end{array}$ \\
\hline \multicolumn{4}{|l|}{$[. . /]$} \\
\hline \multicolumn{4}{|c|}{ [./Checkpoint] } \\
\hline type & Checkpoint & - & $\begin{array}{l}\text { Output for MOOSE recovery } \\
\text { checkpoint files }\end{array}$ \\
\hline file_base & string & - & $\begin{array}{l}\text { Desired solution output name without } \\
\text { extension. If not provided, MOOSE } \\
\text { sets the output file name of master app } \\
\text { to the input file name of master app } \\
\text { and sets the output file name of } \\
\text { subapp to the input file name of } \\
\text { master app plus the subapp name. }\end{array}$ \\
\hline execute_on & $\begin{array}{l}\text { NONE, INITIAL, } \\
\text { LINEAR, } \\
\text { NONLINEAR, } \\
\text { TIMESTEP_END, } \\
\text { TIMESTE_BEGIN, } \\
\text { FINAL, CUSTTOM }\end{array}$ & $\begin{array}{c}\text { INITIAL, } \\
\text { TIMESTEP_EN } \\
\text { D }\end{array}$ & $\begin{array}{l}\text { Indicate when this output should be } \\
\text { executed }\end{array}$ \\
\hline start_step & int & - & $\begin{array}{l}\text { Time step at which this output object } \\
\text { begins to operate }\end{array}$ \\
\hline end_step & int & - & Time step at which this output object \\
\hline
\end{tabular}




\begin{tabular}{|l|l|c|l|}
\hline & & & stop operating \\
\hline start_time & double & - & $\begin{array}{l}\text { Time at which this output object } \\
\text { begins to operate }\end{array}$ \\
\hline end_time & double & - & $\begin{array}{l}\text { Time at which this output object stop } \\
\text { operating }\end{array}$ \\
\hline num_files & unsigned int & 2 & Number of restart files to save \\
\hline$[. . /]$ \\
{[]}
\end{tabular}

\section{E.2. Sample Inputs}

Fig. E.1 presents a sample input for TreeKangaroo, and Fig. E.2 presents a sample input for TreeFrog.

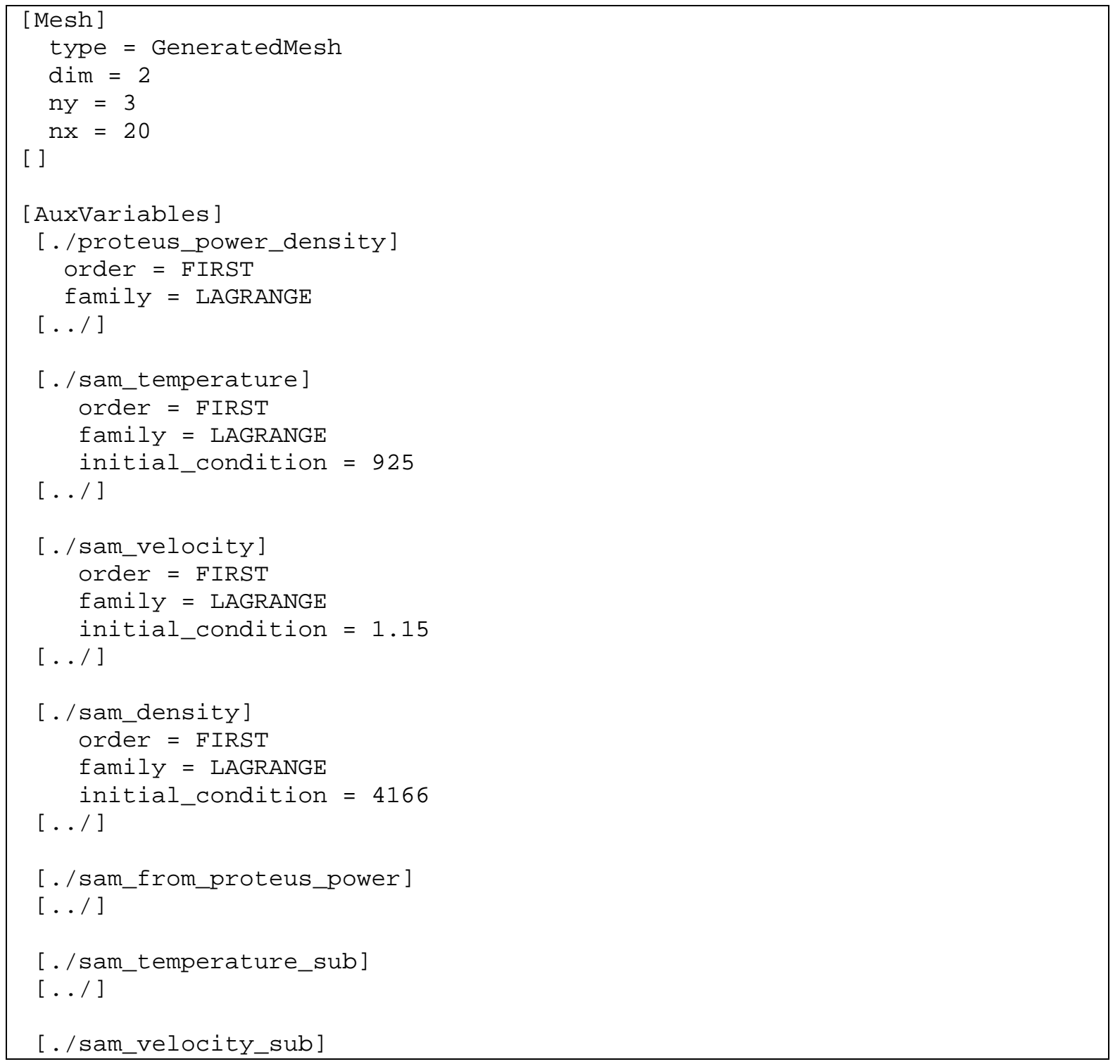




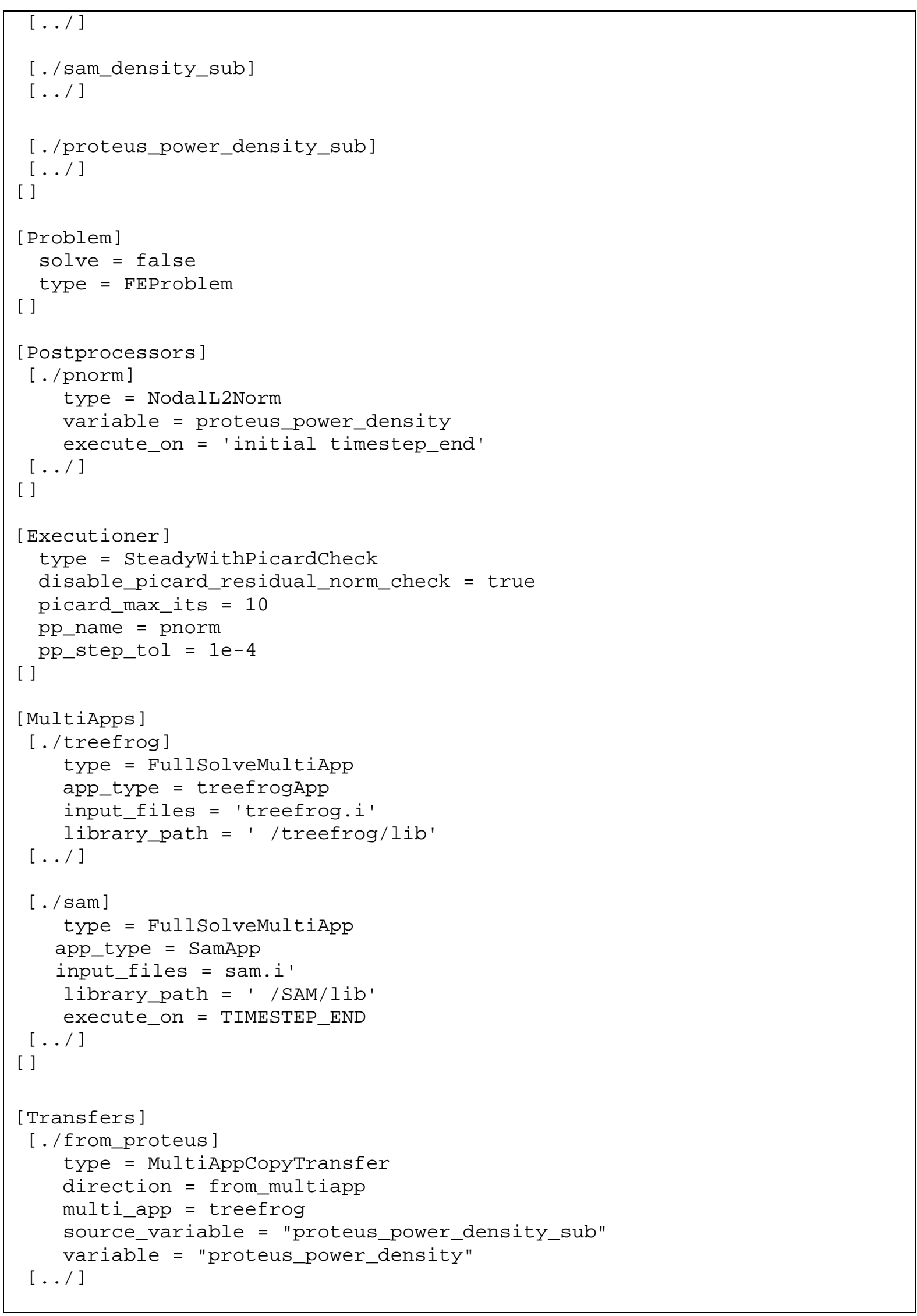




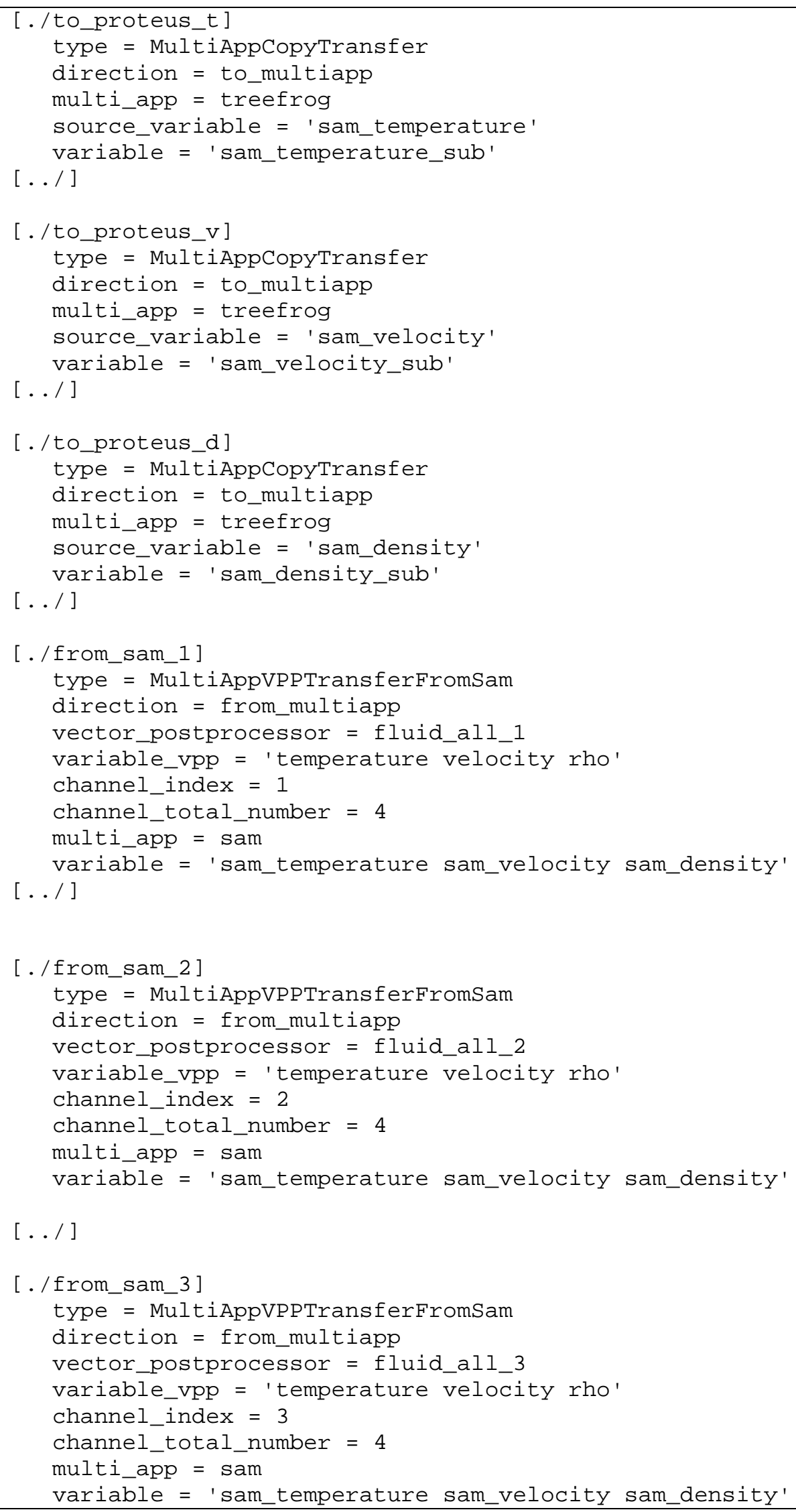




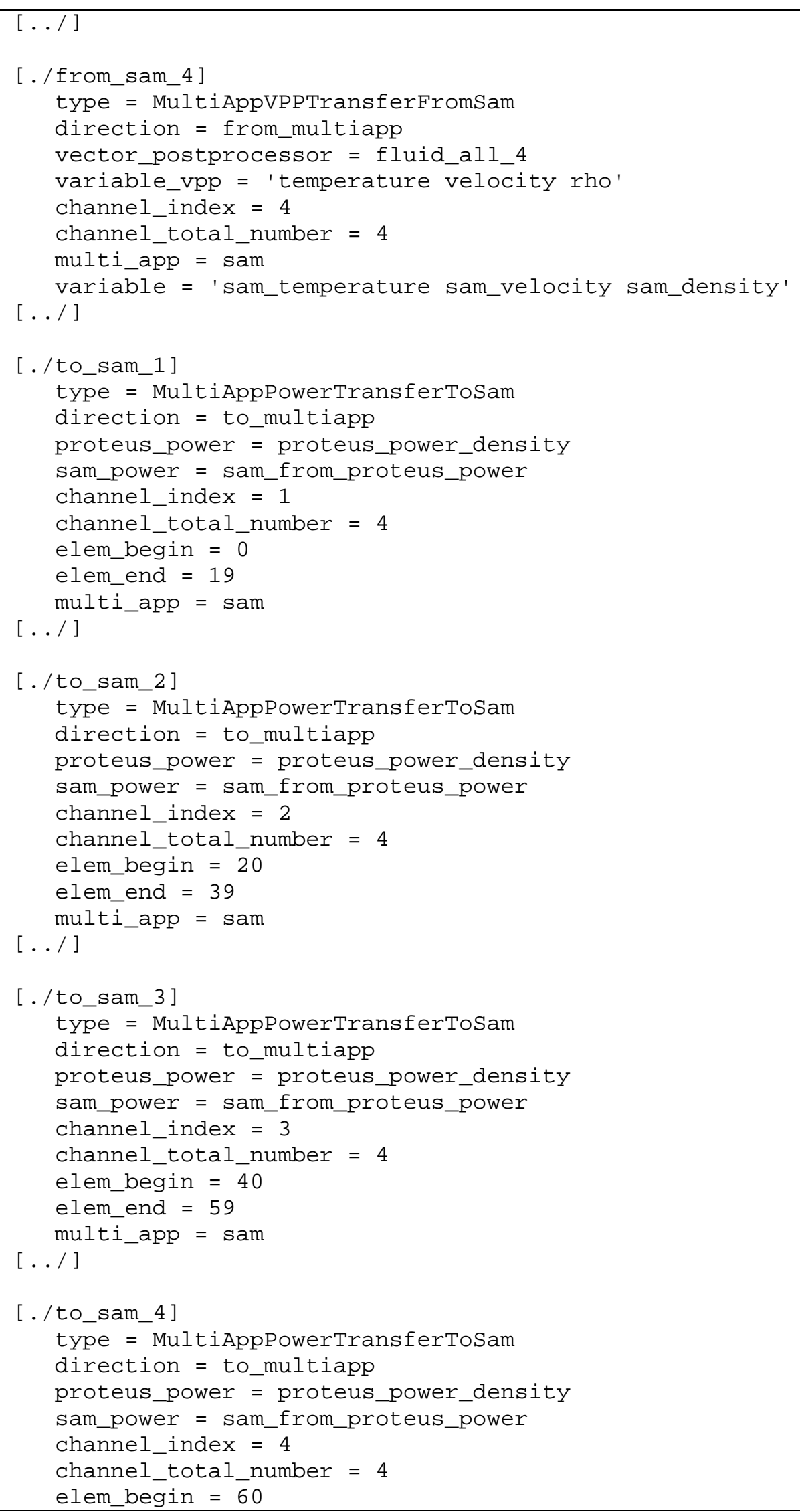




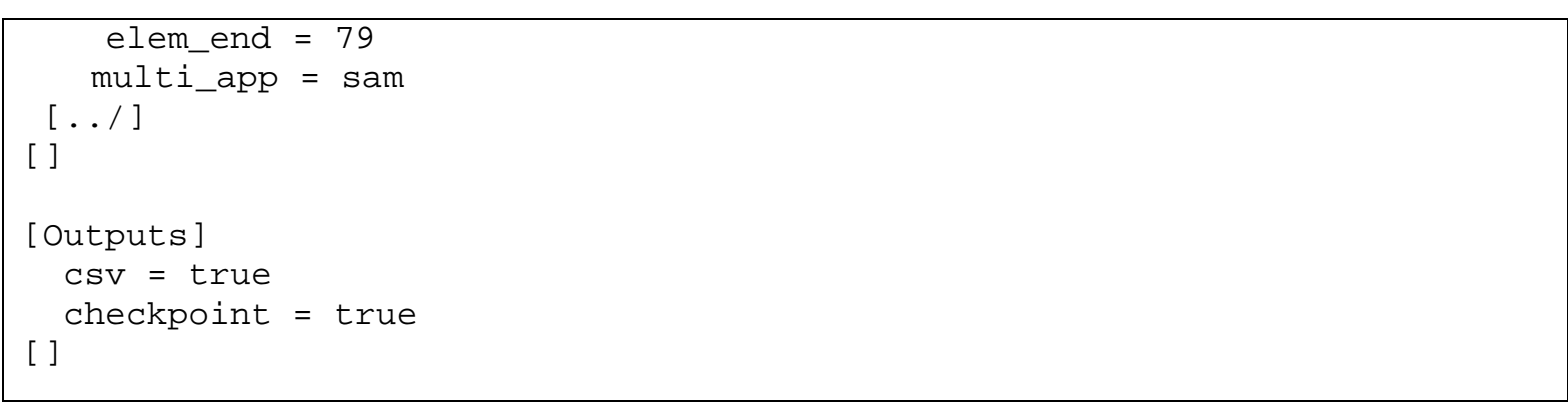

Fig. E.1. Sample input for TreeKangaroo.

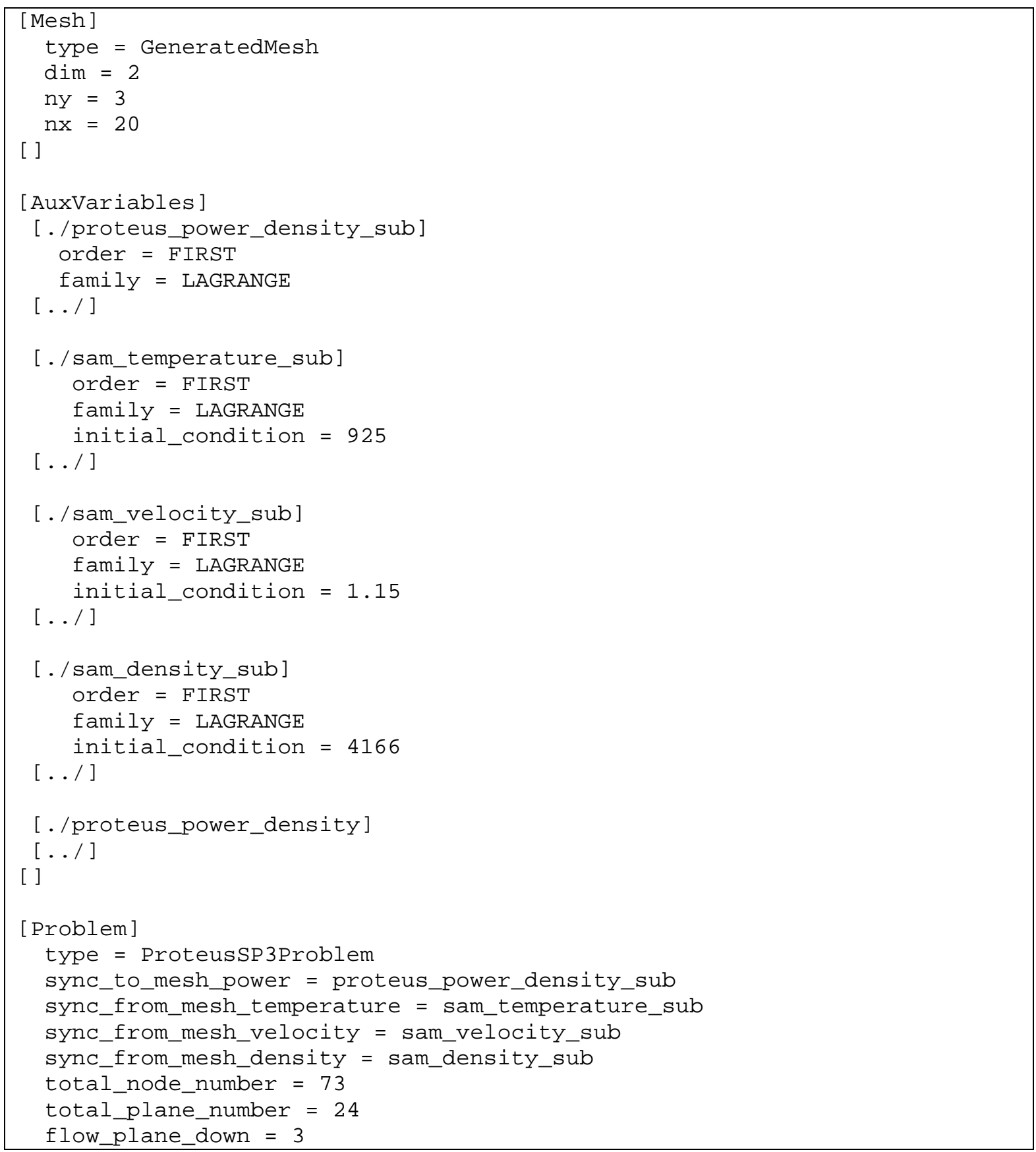




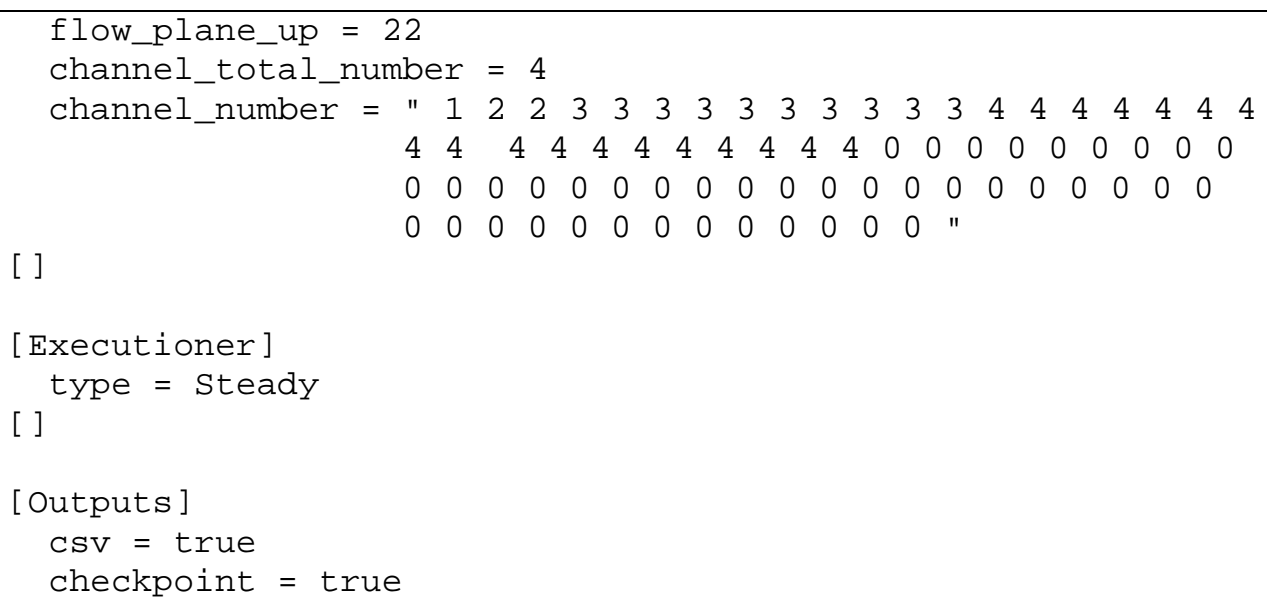

Fig. E.2. Sample input for TreeFrog. 


\section{Argonne}

\section{Nuclear Science and Engineering Division}

Argonne National Laboratory

9700 South Cass Avenue, Bldg. 208

Argonne, IL 60439-4842

www.anl.gov 